\title{
On Multi-agent Reinforcement Learning in Matrix, Stochastic and Differential Games
}

\author{
by \\ Mostafa D. Awheda \\ A thesis submitted to \\ the Faculty of Graduate and Postdoctoral Affairs \\ in partial fulfilment of \\ the requirements for the degree of \\ Doctor of Philosophy \\ in
}

Electrical and Computer Engineering

Ottawa-Carleton Institute for Electrical and Computer Engineering (OCIECE)

Department of Systems and Computer Engineering

Carleton University

Ottawa, Ontario, Canada

September, 2016

Copyright (C)

2016 - Mostafa D. Awheda 
The undersigned hereby recommends to the

Faculty of Graduate and Postdoctoral Affairs

acceptance of the thesis

\section{On Multi-agent Reinforcement Learning in Matrix, Stochastic and Differential Games}

submitted by Mostafa D. Awheda, M.Sc.

in partial fulfillment of the requirements for the degree of

Doctor of Philosophy in Electrical and Computer Engineering

Professor Howard M. Schwartz, Thesis Supervisor,

Department of Systems and Computer Engineering

Professor Jason Gu, External Examiner, Department of Electrical and Computer Engineering, Dalhousie University

Professor Yvan Labiche, Chair, Department of Systems and Computer Engineering

Carleton University

September, 2016 


\section{Abstract}

In this thesis, we investigate reinforcement learning algorithms on matrix, stochastic, and differential games. In matrix and stochastic games, the states and actions are represented in continuous domains. We propose two decentralized multi-agent reinforcement learning algorithms to solve the problem of learning in matrix and stochastic games when the learning agent has only minimum knowledge about the underlying game and the other learning agents. The proposed algorithms are the constant learning rate-based exponential moving average Q-learning (CLREMAQL) algorithm, and the exponential moving average Q-learning (EMAQL) algorithm. We mathematically show that the proposed CLR-EMAQL algorithm converges to Nash equilibrium in games with pure Nash equilibrium. We introduce the concept of Win-or-Learn-Slow (WoLS) mechanism for the proposed EMAQL algorithm so that the proposed algorithm learns fast when it is winning, and learns cautiously when it is losing. We also provide a theoretical proof of convergence to Nash equilibrium for the proposed EMAQL algorithm in games with pure Nash equilibrium. In games with mixed Nash equilibrium, our mathematical analysis shows that the proposed EMAQL algorithm converges to an equilibrium. Although our mathematical analysis does not explicitly show that the proposed EMAQL

algorithm converges to Nash equilibrium, our simulation results indicate that the proposed EMAQL algorithm does converge to Nash equilibrium. Our simulation 
results also show the convergence of the proposed algorithms to Nash equilibrium over a variety of matrix and stochastic games that some of the state-of-the-art multi-agent reinforcement learning algorithms fail to converge to Nash equilibrium at.

In differential games, on the other hand, where the states and actions are represented in continuous domains, we provide four main contributions. First, we propose a new fuzzy reinforcement learning algorithm to solve the problem of learning in differential games. The proposed algorithm makes use of function approximation systems (fuzzy inference systems, FISs) and reinforcement learning methods. The proposed algorithm tunes the input and output parameters of each FIS by using the residual gradient value iteration algorithm. The proposed algorithm is called the residual gradient fuzzy actor critic learning (RGFACL) algorithm. Our simulation results show that the proposed RGFACL algorithm outperforms the state-of-the-art fuzzy reinforcement learning algorithms. Second, we propose a new fuzzy reinforcement learning algorithm for pursuit-evasion games so that the pursuer trained by the proposed algorithm can capture the evader even when the environment of the game is different from the training environment. The proposed algorithm incorporates the future position of the evader predicted by a Kalman filter in the tunning mechanism of the fuzzy logic controller (FLC) of the learning pursuer so that the pursuer moves directly to the expected position of the evader, where the capture of the evader will occur. The proposed algorithm is called the Kalman filter fuzzy actor critic learning (KFFACL) algorithm. Simulation results show the outperformance of the proposed algorithm over the state-of-the-art fuzzy reinforcement learning algorithms. Third, we propose a new decentralized fuzzy reinforcement learning algorithm for multipursuer pursuit-evasion differential games with a single-superior evader that has a speed equal to the speed of the pursuers. The proposed algorithm incorporates a formation control mechanism in the reward function of the learning agent so that the 
superior evader is enclosed by the learning pursuers and can be captured at the end. Our simulation results indicate that the superior evader is always captured by one or some of the pursuers learning their strategies by the proposed algorithm. Fourth, we propose a new decentralized fuzzy reinforcement learning algorithm for multi-pursuer pursuit-evasion differential games with a single-superior evader that has a speed that is equal to or higher than the speed of the pursuers. The proposed algorithm incorporates the Apollonius circles with a developed formation control mechanism in the reward function of the learning pursuer so that the superior evader is surrounded by the pursuers and can be captured at the end. Simulation results show the effectiveness of the proposed algorithm. 


\section{Acknowledgments}

I would like to express my deepest gratitude to my advisor, Professor Howard M. Schwartz, for his guidance, mentorship, encouragement, and friendship through the course of my studies and research. I would also like to thank my thesis committee members for all their valuable comments and suggestions. I would also like to thank my dear friends here in Canada and back home in Libya for their encouragement. My deepest thanks goes to my wife as well as my children, Reham, Wael, Meral, and Mariya, for their unconditional love and support. Finally, I would like to thank my parents, sisters, and brothers for their love, support, and encouragement. I would not have come this far without you all. 


\section{Contents}

Abstract $\quad$ iii

Acknowledgments $\quad$ vi

Table of Contents vii

List of Tables $\quad$ xii

List of Figures xiv

List of Symbols $\quad$ xxi

1 Introduction 1

1.1 Motivation ........................... 1

1.2 Contributions and Publications ................ 4

1.3 Organization of the Thesis . . . . . . . . . . . . . . 10

2 Background and Preliminary Ideas 13

2.1 Introduction . . . . . . . . . . . . . . . . . . . 13

2.2 A Framework for Reinforcement Learning . . . . . . . . . . . . . . . . 13

2.2.1 Markov Decision Processes . . . . . . . . . . . . . . . . 14

2.2.2 Q-learning Algorithm . . . . . . . . . . . . 21

2.2.3 Matrix Games . . . . . . . . . . . . . . . . . . 22 
2.2.4 Stochastic Games . . . . . . . . . . . . . . . . . . 25

2.3 Fuzzy Systems . . . . . . . . . . . . . . . . . . . . . . . . . . 28

2.3.1 Membership Function . . . . . . . . . . . . . . . 29

2.3.2 Fuzzy IF-THEN Rules . . . . . . . . . . . . . . . . . . . . . . 32

2.3.3 Fuzzy Inference Engine . . . . . . . . . . . . . . . . 33

2.3.4 Fuzzification and Defuzzification . . . . . . . . . . . . 34

2.4 Summary and Discussion . . . . . . . . . . . . . . . . 35

\section{Multi-agent Reinforcement Learning in Matrix and Stochastic} Games $\quad 36$

3.1 Introduction . . . . . . . . . . . . . . . . . . 36

3.2 An Overview on Some Multi-agent Reinforcement Learning Algorithms 39

3.2.1 The Policy Gradient Ascent with Approximate Policy Prediction (PGA-APP) Algorithm . . . . . . . . . . . . 39

3.2.2 The Weighted Policy Learner Algorithm . . . . . . . . . . . . 41

3.2.3 Win or Learn Fast Policy Hill-Climbing (WoLF-PHC) Algorithm 43

3.2.4 Summary and Discussion . . . . . . . . . . . . 45

3.3 The Proposed Constant Learning Rate-based Exponential Moving Average Q-Learning (CLR-EMAQL) Algorithm . . . . . . . . . . . . . . 47

3.3.1 The Multi-agent Learning Dynamics of the Proposed CLREMAQL Algorithm . . . . . . . . . . . . . . 50

3.3.2 The Mathematical Analysis of the Proposed CLR-EMAQL Algorithm .......................... 52

3.4 The Proposed Exponential Moving Average Q-Learning (EMAQL) Algorithm .......................... 57

3.4.1 WoLF and WoLS Mechanisms . . . . . . . . . . . . . . 59

3.4.2 Pure Nash Equilibrium . . . . . . . . . . . . . . . . 61 
3.4.3 Mixed Nash Equilibrium . . . . . . . . . . . . . . . 62

3.4.4 Nash Equilibrium $\pi^{*}(s) \ldots \ldots . \ldots . . \ldots 63$

3.4.5 The Mathematical Analysis of the Proposed EMAQL Algorithm 67

3.4.6 The Stability of the Equilibrium Solutions of the Proposed EMAQL Algorithm . . . . . . . . . . . . . . . . 72

3.5 Simulation and Results . . . . . . . . . . . . . . 76

3.5.1 Matrix Games . . . . . . . . . . . . . . . . . 77

3.5.2 Stochastic Games . . . . . . . . . . . . . . . . . . 79

3.6 Summary and Discussion . . . . . . . . . . . . . . . 89

4 Reinforcement Learning in Differential Games $\quad 92$

4.1 Introduction . . . . . . . . . . . . . . . . . . . . 92

4.2 Direct and Residual Gradient Algorithms . . . . . . . . . . . . . . . . 97

4.2.1 The Direct Value Iteration Algorithm . . . . . . . . . . . . . . 98

4.2.2 The Residual Gradient Value Iteration Algorithm . . . . . . . 99

4.3 Fuzzy Inference Systems . . . . . . . . . . . . . . . . . . . . . 100

4.4 The Q-learning Fuzzy Inference System (QLFIS) Algorithm . . . . . 104

4.4.1 Update Rules for the FIS . . . . . . . . . . . . . . . . . . 104

4.4.2 Update Rules for the FLC . . . . . . . . . . . . . . . 105

4.5 The Pursuit-Evasion Game . . . . . . . . . . . . . . . . . . 106

4.6 The Proposed Algorithm . . . . . . . . . . . . . . . . . . . . . . 109

4.6.1 Adaptation Rules for the Critic . . . . . . . . . . . . . . . . 110

4.6.2 Adaptation Rules for the Actor . . . . . . . . . . . . . . . . 125

4.7 Simulation and Results . . . . . . . . . . . . . . . . . 127

4.7 .1 Pursuit-Evasion Game 1 . . . . . . . . . . . . . . . 130

4.7.2 Pursuit-Evasion Game 2 . . . . . . . . . . . . . . . . 131

4.7 .3 Pursuit-Evasion Game 3 . . . . . . . . . . . . . . 133 
4.8 Summary and Discussion . . . . . . . . . . . . . . . . . 135

5 A Fuzzy Reinforcement Learning Algorithm Using a Predictor for $\begin{array}{ll}\text { Pursuit-Evasion Games } & 140\end{array}$

5.1 Introduction . . . . . . . . . . . . . . . . . . . . . 140

5.2 The Proposed Algorithm . . . . . . . . . . . . . . . . . . . . . . . 142

5.2.1 A Kalman Filter Predictor . . . . . . . . . . . . . . . . . . 143

5.2.2 The Proposed KFFACL Algorithm . . . . . . . . . . . 145

5.3 Simulation and Results . . . . . . . . . . . . . . . . . . . . . 148

5.3 .1 Simulation Setup . . . . . . . . . . . . . . . . . . 149

5.3 .2 Results and Analysis . . . . . . . . . . . . . . . . 151

5.4 Summary and Discussion . . . . . . . . . . . . . . . . . 153

6 Decentralized Learning in Multi-Pursuer Pursuit-Evasion Differential Games with a Single Evader Having the Same Speed as the $\begin{array}{ll}\text { Pursuers } & 156\end{array}$

6.1 Introduction . . . . . . . . . . . . . . . 156

6.2 Problem Definition . . . . . . . . . . . . . . . . . 158

6.3 The Proposed Algorithm . . . . . . . . . . . . . . . . . . . . . . 161

6.3.1 The Action $u_{p_{o}}$ of Pursuer $P_{o} \ldots \ldots \ldots \ldots$

6.3.2 The Reward Function of Pursuer $P_{o} \ldots \ldots \ldots$. . . . . . 164

6.4 Simulation and Results . . . . . . . . . . . . . . . . . . . 168

6.5 Summary and Discussion . . . . . . . . . . . . . . . 171

7 A Decentralized Fuzzy Learning Algorithm for Multi-Player Pursuit-Evasion Differential Games with a Single Evader Having a Speed Similar to or Higher Than the Speed of Pursuers 175

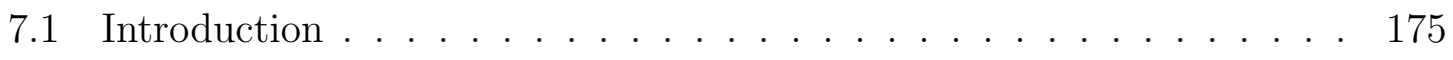


7.2 Preliminary Concepts and Problem Definition . . . . . . . . . 176

7.2.1 Apollonius Circles . . . . . . . . . . . . . . . . . . . 176

$7.2 .2 \quad$ Problem Definition . . . . . . . . . . . . . . . . 179

7.3 The Proposed Algorithm . . . . . . . . . . . . . . . . . . . . 182

7.3.1 The Action $u_{p_{o}}$ of Pursuer $P_{o} \ldots \ldots \ldots \ldots$

7.3.2 The Developed Formation Control Approach . . . . . . . . . . 183

7.3.3 The Reward Function $r_{o}$ of Pursuer $P_{o} \ldots \ldots \ldots$

7.4 Simulation and Results . . . . . . . . . . . . . . . . . . . . . . 189

7.4.1 Simulation Setup . . . . . . . . . . . . . . . 190

7.4 .2 Results. . . . . . . . . . . . . . . . . . . . . 193

7.5 Summary and Discussion . . . . . . . . . . . . . . . . . . 194

8 Conclusions 202

$\begin{array}{ll}\text { List of References } & 219\end{array}$ 


\section{List of Tables}

2.1 The expected rewards and transition probabilities for the finite MDP

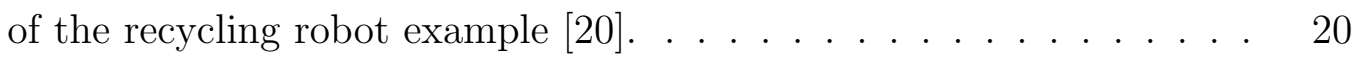

3.1 A comparison among the PGA-APP, the WPL, and the WoLF-PHC algorithms in terms of convergence to Nash equilibrium at some games. 46

4.1 The time (in seconds) that the pursuer trained by each algorithm takes to capture an evader that follows a simple control strategy. The number of episodes here is 200. The evader starts motion at different positions. 131

4.2 The time (in seconds) that the pursuer trained by each algorithm takes to capture an evader that follows an intelligent control strategy. The number of episodes here is 200. The evader starts motion at different positions. . . . . . . . . . . . . . . . . . 133

4.3 The time (in seconds) that the pursuer trained by each algorithm takes to capture an evader that follows an intelligent control strategy. The number of episodes here is 1000 . The evader starts motion at different positions. . . . . . . . . . . . . . . . .

5.1 The time (in seconds) that the pursuer trained by each algorithm takes to capture the evader of Game 1 . The evader starts motion at different

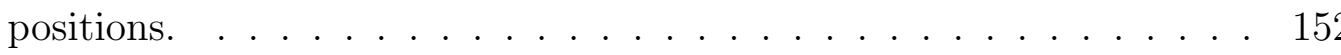


5.2 The time (in seconds) that the pursuer trained by each algorithm takes to capture the evader of Game 2. The evader starts motion at different positions. . . . . . . . . . . . . . . . . 153

5.3 The time (in seconds) that the pursuer trained by each algorithm takes to capture the evader of Game 3. The evader starts motion at different positions. . . . . . . . . . . . . . . . . 153

5.4 The time (in seconds) that the pursuer trained by each algorithm takes to capture the evader of Game 4 . The evader starts motion at different positions. . . . . . . . . . . . . . . . . . . . 154

5.5 The time (in seconds) that the pursuer trained by each algorithm takes to capture the evader of Game 5. The evader starts motion at different positions. . . . . . . . . . . . . . . . . . . 154 


\section{List of Figures}

2.1 Agent-environment interaction in RL [20]. . . . . . . . . . . . . . . . 14

2.2 Graphical description of the Markov decision process (MDP) framework. Not all actions and transitions are shown. . . . . . . . . . . . 16

2.3 Matrix games. . . . . . . . . . . . . . . . . . . . 24

2.4 Two stochastic games $[16] \ldots \ldots \ldots \ldots$. . . . . . . . . 27

2.5 Selected Nash equilibrium paths of grid game $1[16] \ldots$. . . . . . . . 27

2.6 Fuzzy control system architecture [143] . . . . . . . . . . . . . . 29

2.7 A Gaussian MF. . . . . . . . . . . . . . . . . . . 31

2.8 A trapezoidal MF. . . . . . . . . . . . . . . . . . . . . 31

3.1 Two matrix games. . . . . . . . . . . . . . . . . 57

3.2 Probability of selecting actions: (a) Probability of choosing the second action for both players in the coordination game (b) Probability of choosing Player 1's actions in the the matching pennies game. . . . . 58

3.3 Probability of choosing the second action for both players in the coordination game: (a) when using a constant learning rate $\eta_{t}$. (b) when using a decaying learning rate $\eta_{t} \ldots \ldots \ldots \ldots$. . . . . . . .

3.4 Probability of choosing Player 1's actions in the the matching pennies game: (a) when using a constant learning rate $\eta_{t}$. (b) when using a decaying learning rate $\eta_{t} \ldots \ldots \ldots$. . . . . . . . . . . 66

3.5 Matrix games. . . . . . . . . . . . . . . . 78 
3.6 Probability of choosing the second action for both players in the dilemma game. The proposed EMAQL, the PGA-APP, the WPL, and the WoLF-PHC algorithms are shown. . . . . . . . . . .

3.7 Probability of choosing Player 1's actions in the shapley's game. The proposed EMAQL, the PGA-APP, the WPL, and the WoLF-PHC al-

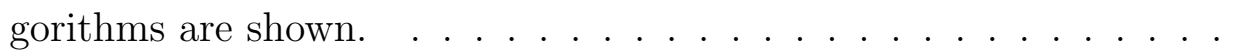

3.8 Probability of choosing the first action for both players in the biased game. The proposed EMAQL, the PGA-APP, the WPL, and the WoLF-PHC algorithms are shown. . . . . . . . . . . . . 82

3.9 Two stochastic games $[16] \ldots \ldots \ldots$. . . . . . . . . . . 83

3.10 (a) A Nash equilibrium of grid game 1. (b) A Nash equilibrium of grid game $2[16] \ldots \ldots \ldots \ldots \ldots \ldots$

3.11 Grid game 1: (a) The probability of selecting action North by Player 1 when learning with the proposed EMAQL, the WoLF-PHC, the PGAAPP, and the WPL algorithms. (b) The probability of selecting action North by Player 2 when learning with the proposed EMAQL, the WoLF-PHC, the PGA-APP, and the WPL algorithms. . . . . . . .

3.12 Grid game 1: (a) The average reward of Player 1 when learning with the proposed EMAQL, the WoLF-PHC, the PGA-APP, and the WPL algorithms. (b) The average reward of Player 2 when learning with the proposed EMAQL, the WoLF-PHC, the PGA-APP, and the WPL algorithms. . . . . . . . . . . . . . . .

3.13 Grid game 2: (a) The probability of selecting action East by Player 1 when learning with the proposed EMAQL, the WoLF-PHC, the PGAAPP, and the WPL algorithms. (b) The probability of selecting action North by Player 2 when learning with the proposed EMAQL, the WoLF-PHC, the PGA-APP, and the WPL algorithms. . . . . . . . 
3.14 Grid game 2: (a) The average reward of Player 1 when learning with the proposed EMAQL, the WoLF-PHC, the PGA-APP, and the WPL algorithms. (b) The average reward of Player 2 when learning with the proposed EMAQL, the WoLF-PHC, the PGA-APP, and the WPL

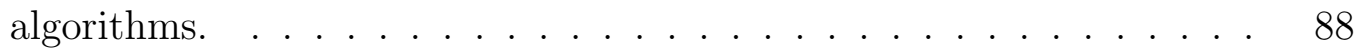

4.1 The QLFIS technique [100]. . . . . . . . . . . . . . . . . 104

4.2 Pursuit-evasion model. . . . . . . . . . . . . . . . . . . . . 107

4.3 The proposed RGFACL algorithm. . . . . . . . . . . 126

4.4 The paths of the pursuer and the evader when learning by the FACL algorithm proposed in [99] (starred lines) against the paths of the pursuer and the evader when following the classical strategy defined in Eq. (4.28) and Eq. (4.29) (dotted lines). The number of episodes used here is 200 .

4.5 The paths of the pursuer and the evader when learning by the QLFIS algorithm proposed in [100] (starred lines) against the paths of the pursuer and the evader when following the classical strategy defined in Eq. (4.28) and Eq. (4.29) (dotted lines). The number of episodes used here is $200 . \ldots \ldots \ldots \ldots \ldots$

4.6 The paths of the pursuer and the evader when learning by the proposed RGFACL algorithm (starred lines) against the paths of the pursuer and the evader when following the classical strategy defined in Eq. (4.28) and Eq. (4.29) (dotted lines). The number of episodes used here is 200. 136

4.7 The paths of the pursuer and the evader when learning by the FACL algorithm proposed in [99] (starred lines) against the paths of the pursuer and the evader when following the classical strategy defined in Eq. (4.28) and Eq. (4.29) (dotted lines). The number of episodes used here is 500 . 
4.8 The paths of the pursuer and the evader when learning by the QLFIS algorithm proposed in [100] (starred lines) against the paths of the pursuer and the evader when following the classical strategy defined in Eq. (4.28) and Eq. (4.29) (dotted lines). The number of episodes used here is $500 . \ldots \ldots \ldots \ldots$. . . . . . . . . . . . . . . 137

4.9 The paths of the pursuer and the evader when learning by the proposed RGFACL algorithm (starred lines) against the paths of the pursuer and the evader when following the classical strategy defined in Eq. (4.28) and Eq. (4.29) (dotted lines). The number of episodes used here is 500. 138

5.1 Pursuit-evasion model with the predicted position of the evader. . . . 146

5.2 The proposed KFFACL algorithm. . . . . . . . . . . . . . . . 148

6.1 Geometric illustration of capture $[72] \ldots \ldots$. . . . . . . . . . . 161

6.2 Geometric illustration of defining the action for the pursuer $P_{o}$. . . . 164

6.3 Geometric illustration of the reward function mechanism for the pursuer $P_{o} . \ldots \ldots \ldots \ldots$. . . . . . . . . . . . . . . . . 168

6.4 The paths of the pursuers (thin-line paths) when each pursuer $P_{o}$ learns its control strategy by the proposed algorithm; the path of the evader (thick-line path) when the evader learns its control strategy by the RGFACL algorithm. The target of the evader here is the position $(x, y)=(-500,-500)$.

6.5 The paths of the pursuers (thin-line paths) when each pursuer $P_{o}$ learns its control strategy by the proposed algorithm; the path of the evader (thick-line path) when the evader learns its control strategy by the RGFACL algorithm. The target of the evader here is the position $(x, y)=(-500,0) \ldots \ldots \ldots \ldots \ldots \ldots$ 
6.6 The paths of the pursuers (thin-line paths) when each pursuer $P_{o}$ learns its control strategy by the proposed algorithm; the path of the evader (thick-line path) when the evader learns its control strategy by the RGFACL algorithm. The target of the evader here is the position $(x, y)=(500,500) \ldots \ldots \ldots \ldots \ldots \ldots \ldots$

6.7 The paths of the pursuers (thin-line paths) when each pursuer $P_{o}$ learns its control strategy by the proposed algorithm; the path of the evader (thick-line path) when the evader learns its control strategy by the RGFACL algorithm. The target of the evader here is the position $(x, y)=(500,0) \ldots \ldots \ldots \ldots \ldots \ldots \ldots$

7.1 Apollonius circle created by the pursuer P and the evader E. . . . . . 179

7.2 Geometric illustration of the reward function mechanism for the pursuer $P_{o} . \ldots \ldots \ldots \ldots$. . . . . . . . . . . . . . . . . 188

7.3 The paths of the pursuers of Game 1 (thin-line paths) when each pursuer $P_{o}$ learns its control strategy by the proposed algorithm; the path of the evader (thick-line path) when it learns its control strategy by the RGFACL algorithm. The target of the evader here is the position $\left(x_{e}^{T}, y_{e}^{T}\right)=(500,500) \ldots \ldots \ldots \ldots \ldots$

7.4 The paths of the pursuers of Game 1 (thin-line paths) when each pursuer $P_{o}$ learns its control strategy by the proposed algorithm; the path of the evader (thick-line path) when it learns its control strategy by the RGFACL algorithm. The target of the evader here is the position $\left(x_{e}^{T}, y_{e}^{T}\right)=(500,0) \ldots \ldots \ldots \ldots \ldots$ 
7.5 The paths of the pursuers of Game 1 (thin-line paths) when each pursuer $P_{o}$ learns its control strategy by the proposed algorithm; the path of the evader (thick-line path) when it learns its control strategy by the RGFACL algorithm. The target of the evader here is the position $\left(x_{e}^{T}, y_{e}^{T}\right)=(-500,500) \ldots \ldots \ldots \ldots \ldots \ldots$

7.6 The paths of the pursuers of Game 1 (thin-line paths) when each pursuer $P_{o}$ learns its control strategy by the proposed algorithm; the path of the evader (thick-line path) when it learns its control strategy by the RGFACL algorithm. The target of the evader here is the position $\left(x_{e}^{T}, y_{e}^{T}\right)=(-500,0)$

7.7 The paths of the pursuers of Game 2 (thin-line paths) when each pursuer $P_{o}$ learns its control strategy by the proposed algorithm; the path of the evader (thick-line path) when it learns its control strategy by the RGFACL algorithm. The target of the evader here is the position $\left(x_{e}^{T}, y_{e}^{T}\right)=(500,500) \ldots \ldots \ldots \ldots \ldots \ldots \ldots \ldots \ldots \ldots \ldots \ldots \ldots$

7.8 The paths of the pursuers of Game 2 (thin-line paths) when each pursuer $P_{o}$ learns its control strategy by the proposed algorithm; the path of the evader (thick-line path) when it learns its control strategy by the RGFACL algorithm. The target of the evader here is the position $\left(x_{e}^{T}, y_{e}^{T}\right)=(500,0)$.

7.9 The paths of the pursuers of Game 2 (thin-line paths) when each pursuer $P_{o}$ learns its control strategy by the proposed algorithm; the path of the evader (thick-line path) when it learns its control strategy by the RGFACL algorithm. The target of the evader here is the position $\left(x_{e}^{T}, y_{e}^{T}\right)=(-500,500) \ldots \ldots \ldots \ldots \ldots \ldots$ 
7.10 The paths of the pursuers of Game 2 (thin-line paths) when each pursuer $P_{o}$ learns its control strategy by the proposed algorithm; the path of the evader (thick-line path) when it learns its control strategy by the RGFACL algorithm. The target of the evader here is the position $\left(x_{e}^{T}, y_{e}^{T}\right)=(-500,0) \ldots \ldots \ldots \ldots \ldots$

7.11 The paths of the pursuers of Game 3 (thin-line paths) when each pursuer $P_{o}$ learns its control strategy by the proposed algorithm; the path of the evader (thick-line path) when it learns its control strategy by the RGFACL algorithm. The target of the evader here is the position $\left(x_{e}^{T}, y_{e}^{T}\right)=(500,500) \ldots \ldots \ldots \ldots \ldots$

7.12 The paths of the pursuers of Game 3 (thin-line paths) when each pursuer $P_{o}$ learns its control strategy by the proposed algorithm; the path of the evader (thick-line path) when it learns its control strategy by the RGFACL algorithm. The target of the evader here is the position $\left(x_{e}^{T}, y_{e}^{T}\right)=(500,0) \ldots \ldots \ldots \ldots \ldots$

7.13 The paths of the pursuers of Game 3 (thin-line paths) when each pursuer $P_{o}$ learns its control strategy by the proposed algorithm; the path of the evader (thick-line path) when it learns its control strategy by the RGFACL algorithm. The target of the evader here is the position $\left(x_{e}^{T}, y_{e}^{T}\right)=(-500,500) \ldots \ldots \ldots \ldots \ldots$

7.14 The paths of the pursuers of Game 3 (thin-line paths) when each pursuer $P_{o}$ learns its control strategy by the proposed algorithm; the path of the evader (thick-line path) when it learns its control strategy by the RGFACL algorithm. The target of the evader here is the position $\left(x_{e}^{T}, y_{e}^{T}\right)=(-500,0) \ldots \ldots \ldots \ldots \ldots$ 


\section{List of Symbols}

\begin{tabular}{ll}
\hline Symbols & Definition \\
\hline \hline$s$ & environment's state \\
$a$ & environment's state space \\
$A$ & agent's action \\
$\operatorname{Tr}(s, a, s)$ & agent's action space \\
& probability of transition to state $s$ when agent is at state $s$ \\
$R$ & and takes action $a$ \\
$\pi^{j}$ & reward function \\
$j$ & policy of agent (player) $j$ \\
$r_{t+1}$ & agent (player) $j$ in a game \\
$\mathcal{R}_{t}$ & immediate reward of the learning agent \\
$t$ & discounted return \\
& current time step \\
&
\end{tabular}




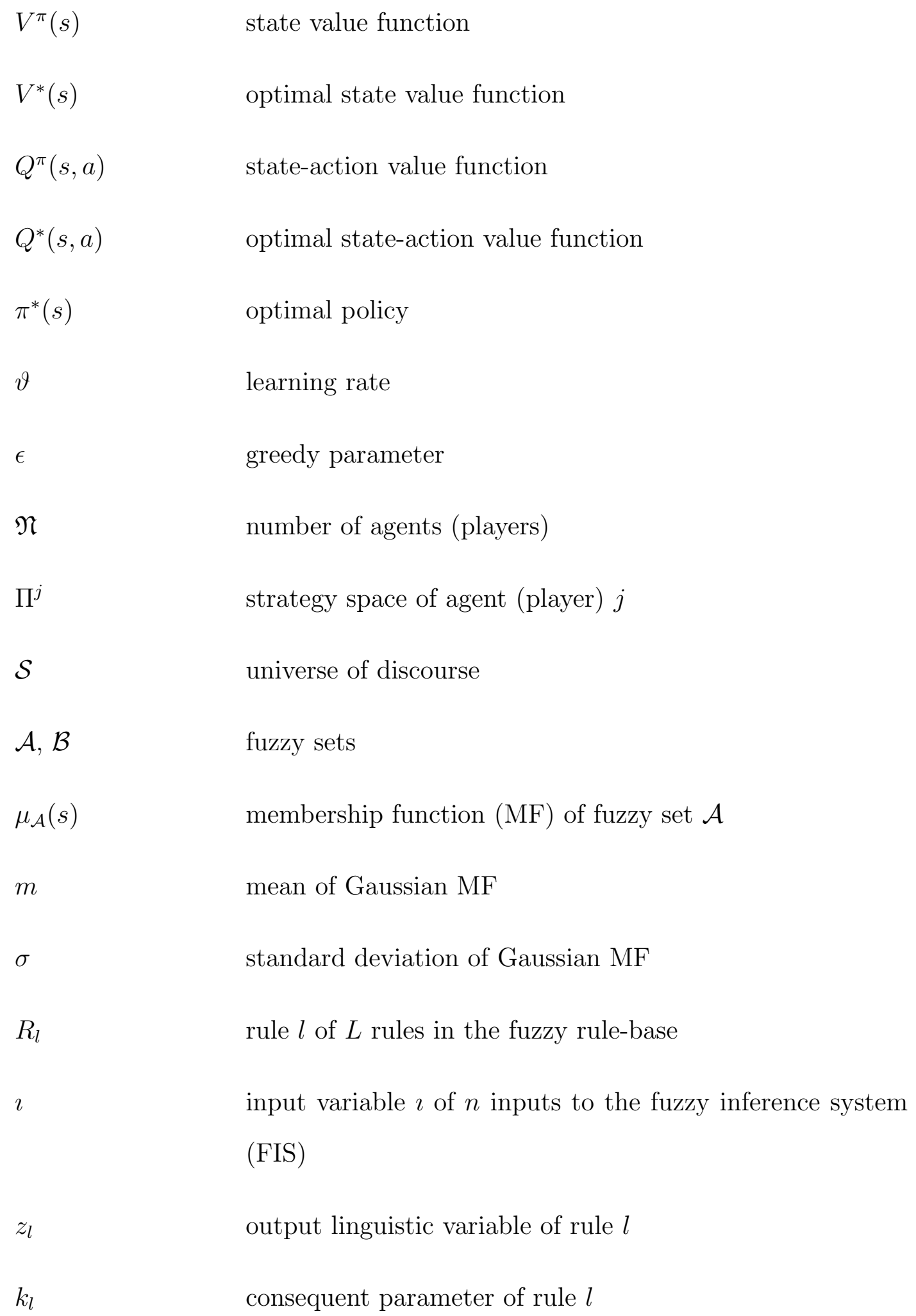




\begin{tabular}{|c|c|}
\hline$Z(\mathbf{s})$ & output of the FIS at state $\mathbf{s}$ \\
\hline$\omega_{l}$ & firing strength of rule $l$ \\
\hline$\Phi_{l}$ & normalized firing strength of rule $l$ \\
\hline$\eta^{P}, \gamma^{P}, \eta^{W}, \delta_{w}, \delta_{l}$ & learning rates \\
\hline$\eta_{c}$ & constant learning rate \\
\hline$\eta_{t}$ & decaying learning rate \\
\hline$\eta_{w}, \eta_{l}$ & learning rates \\
\hline$\eta_{l}^{W o L F}$ & $\eta_{l}$ of WoLF mechanism \\
\hline$\eta_{l}^{W o L S}$ & $\eta_{l}$ of WoLS mechanism \\
\hline$\pi^{j}\left(s_{t}\right)$ & policy of agent (player) $j$ \\
\hline$\pi^{-j}\left(s_{t}\right)$ & policy of agent (player) $j$ 's opponent \\
\hline $\mathfrak{M}$ & number of actions of agent (player) $j$ \\
\hline$\iota$ & current number of episodes \\
\hline$V_{t}\left(\mathbf{s}_{t}\right), V_{t}\left(\mathbf{s}_{t+1}\right)$ & state value functions at states $\mathbf{s}_{t}$ and $\mathbf{s}_{t+1}$, respectively \\
\hline$\Delta_{t}$ & temporal difference error \\
\hline$E$ & mean square error \\
\hline$\psi^{C}$ & input and output parameters of the critic \\
\hline$\psi^{A}$ & input and output parameters of the actor \\
\hline$\Omega\left(s_{\imath}\right)$ & set of parameters of the MF of each input $s_{\imath}$ \\
\hline
\end{tabular}




\begin{tabular}{|c|c|}
\hline$\Omega\left(\omega_{l}\right)$ & set of parameters of each firing strength $\omega_{l}$ \\
\hline$\rho, \rho^{Q}, \rho^{u}$ & learning rates \\
\hline$\kappa$ & represents the pursuer " $p$ " and the evader " $e$ " \\
\hline$V_{\kappa}$ & robot $\kappa$ 's speed \\
\hline$\theta_{\kappa}$ & orientation of robot $\kappa$ \\
\hline$\left(x_{\kappa}, y_{\kappa}\right)$ & position of robot $\kappa$ \\
\hline$L_{\kappa}$ & wheelbase of robot $\kappa$ \\
\hline$u_{\kappa}$ & robot $\kappa$ 's steering angle \\
\hline$\delta_{\kappa}$ & $\begin{array}{l}\text { angle difference between the direction of robot } \kappa \text { and the } \\
\text { pursuer's line-of-sight (LoS) to the evader }\end{array}$ \\
\hline $\boldsymbol{x}(t)$ & position and velocity vector of the evader \\
\hline$w_{x}(t), w_{y}(t)$ & $\begin{array}{l}\text { zero-mean white Gaussian process noise with variances } \sigma_{w_{x}}^{2} \\
\text { and } \sigma_{w_{y}}^{2} \text {, respectively }\end{array}$ \\
\hline $\boldsymbol{y}(t)$ & position components vector of the evader \\
\hline$v_{x}(t)$ and $v_{y}(t)$ & $\begin{array}{l}\text { zero-mean white Gaussian measurement noise with vari- } \\
\text { ances } \sigma_{v_{x}}^{2} \text { and } \sigma_{v_{y}}^{2} \text {, respectively }\end{array}$ \\
\hline$Q_{k f}$ & process noise covariance matrix \\
\hline$R_{k f}$ & observation noise covariance matrix \\
\hline
\end{tabular}




$\begin{array}{ll}\hat{\delta}_{p} & \text { predicted angle difference between the directions of the pur- } \\ & \text { suer and its LoS vector to the evader at its estimated future } \\ & \text { location }\left(\hat{x}_{e}, \hat{y}_{e}\right) \\ & \text { predicted state } \\ \hat{\mathbf{s}}_{t+1} & \text { pursuer of } N \text { pursuers } \\ P_{o} & \text { angle difference between the direction of pursuer } P_{o} \text { and } \\ \alpha_{o} & \text { the direction of } P_{o}^{\prime} \text { 's LOS to evader E } \\ \beta_{o} & \text { angle difference between the direction of evader } E \text { and the } \\ & \text { direction of } E \text { 's LOS to pursuer } P_{o} \\ \epsilon_{o}, V_{p_{o}} & \text { speeds of evader } E \text { and pursuer } P_{o}, \text { respectively } \\ \theta_{\alpha_{o}} & \text { arientation of pursuer } P_{o} \\ u_{p_{o}} & \text { action of pursuer } P_{o} \\ & \end{array}$




\section{Chapter 1}

\section{Introduction}

\subsection{Motivation}

Multi-agent learning algorithms have received considerable attention over the past two decades $[11,29]$. Several multi-agent reinforcement learning (MARL) algorithms have recently been proposed and studied $[1,8,9,14,16,19,22,40-42]$. Some of these algorithms have theoretical results of convergence in general-sum games. In addition, some of these algorithms fail to converge to Nash equilibrium in some games. Due to the complexity of the problem, the majority of these algorithms make some assumptions so as to guarantee convergence of the learning agents to Nash equilibrium. For example, some of these algorithms assume that each learning agent either has some knowledge of the underlying game (such as knowing the Nash equilibrium of the game) or knows the immediate actions and rewards of the other learning agents. Other algorithms assume that the policies of the other learning agents (the learning agent's opponents) are also known by the learning agent. Such strict assumptions may limit the use of these algorithms because the underlying game structure (Nash equilibrium), and the policies and rewards of the learning agent's opponents are often unknown to the learning agent and may be learned via interacting with the other learning agents [9]. In a multi-agent 
environment, agents may have independent goals and do not know the underlying game. In addition, they may not know any information about each other. Moreover, they may not share any information with each other as sharing information may not be applicable in real applications. Among multi-agent learning algorithms, decentralized learning algorithms have become an attractive research field. In decentralized learning, there is no central learning strategy for all the learning agents. Instead, each agent is responsible to learn its own strategy. Decentralized learning algorithms are used in the literature to learn the Nash equilibrium of games with incomplete information [28,30-32]. That is, they are used when each learning agent in the game lacks the information about the policies, immediate actions and immediate rewards of the other agents. Therefore, we want to propose a decentralized reinforcement learning algorithm so that each learning agent can learn its Nash equilibrium strategy. The proposed decentralized reinforcement learning algorithm should guarantee the convergence of the learning agent to a Nash equilibrium strategy.

Reinforcement learning has attracted attention and has been used in intelligent robot control systems $[103,104,115-118,126]$. Reinforcement learning has also been used for solving nonlinear optimal control problems [88-92, 106-112]. Most of the traditional reinforcement learning algorithms represent the state/state-action value function as a look-up table for each state/state-action-pair [20]. Despite the theoretical foundations of these algorithms and their effectiveness in many applications, these reinforcement learning approaches cannot be applied to differential games, where the states and actions are continuous. One of the possible solutions to the problem of continuous domains is to discretize the state and action spaces. However, the discretization of continuous domains may lead to the problem of the curse of dimensionality that appears when discretizing large continuous states and/or actions $[3,20,126]$. In many real-world applications, a priori discretization of the state 
and action spaces may not be useful [84]. Moreover, using a coarse discretization of the state or action space may lead to a poor performance [85-87]. To overcome the problem of learning in differential games, one may use a function approximation system to represent the large discrete and/or continuous spaces [20, 99, 123, 141]. Therefore, we want to propose a new technique that uses function approximation systems with reinforcement learning methods to solve the problem of learning in differential games.

In a pursuit-evasion differential game, the pursuer learning its control strategy by any learning algorithm usually captures the evader when the environment of the game is similar to the environment that the pursuer was trained on. However, the trained pursuer may not be able to capture the evader if the environment of the pursuit-evasion differential game is different from the training environment. In the real field, the environment may be different from the training environment. That is, the trained pursuer may be faced with a new and different evader with different parameters. The performance of the trained robot may be degraded if the trained robot is used in an environment that is different from the one that the robot was trained on [137]. Our concern stands from the case when the trained pursuer faces a different unexpected evader. Would the trained pursuer be able to capture that unexpected evader? Therefore, we want to propose a new fuzzy reinforcement learning algorithm so that the ability of the trained pursuer to capture the evader in a pursuit-evasion differential game increases even when the environment of the game is different from the environment the pursuer was trained on.

A number of articles $[54,61,63-65,100]$ investigated pursuit-evasion games with slow evaders, where the capture of the evader is always guaranteed. However, in real-world 
applications, evaders may run with speed similar to or higher than the speed of pursuers. In such cases, the capture of the faster (superior) evader may require more than one pursuer. This has led researchers to investigate multi-pursuer single-superiorevader pursuit-evasion games and propose a number of different techniques so that one or some of the pursuers can capture the superior evader [72-75, 78-80, 82, 83]. These techniques use different mechanisms such as hierarchical decomposition approaches and formation control approaches to tune the pursuers. However, all these techniques are deterministic approaches and involve no learning in the tuning mechanisms of the pursuers. In real-world applications, pursuers may need to adapt to changing environments. Therefore, we want to propose a fuzzy reinforcement learning algorithm to solve the problem of learning in multi-pursuer single-superiorevader pursuit-evasion differential games. Our objective is to make the pursuers in the multi-pursuer single-superior-evader pursuit-evasion differential game learn their strategies so that one or some of the learning pursuers can capture the superior evader.

\subsection{Contributions and Publications}

1. We propose two decentralized reinforcement learning algorithms to solve the problem of learning in matrix and stochastic games when:

- each learning agent does not have enough information about the underlying game (i.e. each learning agent does not know the Nash equilibrium of the game);

- each learning agent is oblivious to the other agents. That is,

- each learning agent does not share information with the other learning agents; 
- each learning agent does not know the other learning agents' immediate rewards and actions in case of matrix games, and does not know the other learning agents' immediate rewards in case of stochastic games.

The proposed algorithms use the Exponential Moving Average (EMA) approach along with the Q-learning algorithm as a basis to update the policy for the learning agent so that the agent's policy converges to a Nash equilibrium policy. Two multi-agent policy iteration learning algorithms are proposed in this work. The first proposed algorithm uses a constant learning rate when updating the policy of the learning agent, while the second proposed algorithm uses two different decaying learning rates. These two decaying learning rates are updated based on either the Win-or-Learn-Fast (WoLF) mechanism or the Win-or-Learn-Slow (WoLS) mechanism. The WoLS mechanism is introduced to make the algorithm learn fast when it is winning and learn cautiously when it is losing. The second proposed algorithm uses the rewards received by the learning agent to decide which mechanism (WoLF mechanism or WoLS mechanism) to use for the game being learned. The proposed algorithms have been theoretically analyzed and a mathematical proof of convergence to pure Nash equilibrium is provided for each algorithm. In the case of games with mixed Nash equilibrium, our mathematical analysis shows that the second proposed algorithm converges to an equilibrium. Although our mathematical analysis does not explicitly show that the second proposed algorithm converges to a Nash equilibrium, our simulation results indicate that the second proposed algorithm does converge to Nash equilibrium. The proposed algorithms are examined on a variety of matrix and stochastic games. Simulation results show that the second proposed algorithm converges in a wider variety of situations than state-of-the-art multi-agent reinforcement learning (MARL) algorithms. 
2. We propose a new fuzzy reinforcement learning algorithm to solve the problem of learning in differential games that have continuous state and action spaces. The proposed algorithm makes use of function approximations with reinforcement learning methods. The proposed algorithm uses the residual gradient value iteration algorithm to tune the input and output parameters of each function approximation system. The proposed algorithm is called the residual gradient fuzzy actor critic learning (RGFACL) algorithm. The proposed algorithm is used to learn three different pursuit-evasion differential games. Simulation results show that the performance of the proposed RGFACL algorithm outperforms the performance of the state-of-the-art fuzzy reinforcement learning algorithms in terms of convergence and speed of learning.

3. We propose a new fuzzy reinforcement learning algorithm for pursuit-evasion games. The pursuer trained by the proposed algorithm can capture the evader not only when the environment of the game is similar to the training environment, but also when the environment of the game is different from the training environment. The proposed algorithm predicts the future position of the evader using a Kalman filter and then tunes the fuzzy logic controller (FLC) of the pursuer so that the pursuer moves directly to the expected position of the evader, where the capture of the evader will occur. The proposed algorithm is called the Kalman filter fuzzy actor critic learning (KFFACL) algorithm. The proposed KFFACL algorithm is applied to pursuit-evasion differential games that have environments different from the training environment. Simulation results show that the proposed KFFACL algorithm outperforms the state-of-the-art fuzzy reinforcement learning algorithms in terms of the ability of the pursuer to capture the evader and the capture time.

4. We propose two fuzzy reinforcement learning algorithms to solve the problem of learning in multi-pursuer single-superior-evader pursuit-evasion differential games: 
- the first proposed algorithm is applicable to multi-pursuer single-superior-evader pursuit-evasion differential games where the evader has a speed that is similar to the speed of each pursuer. Each pursuer of the game uses the proposed algorithm to learn its control strategy. The proposed algorithm of each pursuer uses the residual gradient fuzzy actor critic learning (RGFACL) algorithm to tune the parameters of the fuzzy logic controller (FLC) of the pursuer. The proposed algorithm uses a formation control approach in the tuning mechanism of the FLC of the learning pursuer so that the learning pursuer or the other learning pursuers can capture the superior evader. The formation control mechanism used by the proposed algorithm guarantees that the pursuers are distributed around the superior evader in order to avoid collision between pursuers. The formation control mechanism also guarantees that the capture regions of each two adjacent pursuers overlap or at least border each other so that the capture of the superior evader will be guaranteed. The proposed algorithm is a decentralized algorithm as no communication among pursuers is required. The only information that the proposed algorithm of each learning pursuer requires is the position and the speed of the superior evader. The proposed algorithm is used to learn a multi-pursuer single-superior-evader pursuit-evasion differential game. The simulation results show the effectiveness of the proposed algorithm as the superior evader is always captured by one or some of the pursuers learning their strategies by the proposed algorithm;

- the second proposed algorithm is applicable to multi-pursuer single-superiorevader pursuit-evasion differential games where the evader has a speed that is similar to or higher than the speed of each pursuer. The proposed algorithm uses the well-known Apollonius circle mechanism to define the capture region of the learning pursuer based on its location and the location of the superior 
evader. The proposed algorithm uses the Apollonius circle with a newly developed formation control approach in the tuning mechanism of the fuzzy logic controller (FLC) of the learning pursuer so that one or some of the learning pursuers can capture the superior evader. The formation control mechanism used by the proposed algorithm guarantees that the pursuers are distributed around the superior evader in order to avoid collision between pursuers. The formation control mechanism used by the proposed algorithm also makes the Apollonius circles of each two adjacent pursuers intersect or be at least tangent to each other so that the capture of the superior evader can occur. The proposed algorithm is a decentralized algorithm as no communication among the pursuers is required. The only information the proposed algorithm requires is the position and the speed of the superior evader. The proposed algorithm is used to learn different multi-pursuer single-superior-evader pursuit-evasion differential games. The simulation results show the effectiveness of the proposed algorithm.

The related publications are listed as follows:

1. M. D. Awheda and H. M. Schwartz, "Exponential moving average Q-learning algorithm", In Adaptive Dynamic Programming And Reinforcement Learning (ADPRL), 2013 IEEE Symposium on, pp. 31-38. IEEE, 2013.

2. M. D. Awheda and H. M. Schwartz, "Exponential moving average based multiagent reinforcement learning algorithms", Artificial Intelligence Review, 45.3, pp. 299-332, 2016.

3. M. D. Awheda and H. M. Schwartz, "The residual gradient FACL algorithm for differential games", Proceedings of the 28th IEEE Canadian Conference on Electrical 
and Computer Engineering (CCECE 2015), Halifax, Nova Scotia, Canada, May 3-6, 2015.

4. M. D. Awheda and H. M. Schwartz, "A residual gradient fuzzy reinforcement learning algorithm for differential games", International Journal of Fuzzy Systems, Accepted for publication, October 2016.

5. M. D. Awheda and H. M. Schwartz, "A fuzzy reinforcement learning algorithm using a predictor for pursuit-evasion games", Proceedings of the 2016 IEEE International Systems Conference, Orlando, Florida, April 18-21, 2016.

6. M. D. Awheda and H. M. Schwartz, "A fuzzy reinforcement learning algorithm with a prediction mechanism", Proceedings of The 22nd Mediterranean Conference on Control and Automation (MED), Palermo, Italy, June 16-19, 2014.

7. M. D. Awheda and H. M. Schwartz, "Decentralized learning in pursuit-evasion differential games with multi-pursuer and single-superior evader", Proceedings of the 2016 IEEE International Systems Conference, Orlando, Florida, April 18-21, 2016.

8. M. D. Awheda and H. M. Schwartz, "A decentralized fuzzy learning algorithm for pursuit-evasion differential games with superior evaders", Journal of Intelligent and Robotic Systems, 83.1, pp. 35-53, 2016. 


\subsection{Organization of the Thesis}

The outline of this thesis is as follows:

\section{Chapter 2 - Background and Preliminary Ideas}

We briefly review some background information on reinforcement learning and fuzzy systems. This chapter presents the introductory concepts which form the fundamental background for the work presented in the subsequent chapters.

\section{Chapter 3 - Multi-agent Reinforcement Learning in Matrix and Stochastic}

\section{Games}

We propose two multi-agent reinforcement learning algorithms in this chapter; the constant learning rate-based exponential moving average Q-learning (CLR-EMAQL) algorithm, and the exponential moving average Q-learning (EMAQL) algorithm. We introduce the concept of Win-or-Learn-Slow (WoLS) mechanism so that the proposed EMAQL algorithm learns cautiously when it is losing. We also provide theoretical proofs of convergence to Nash equilibrium strategies for the proposed algorithms. We present simulation results that show the convergence of the proposed algorithms to Nash equilibrium strategies.

\section{Chapter 4 - Reinforcement Learning in Differential Games}

In this chapter, we propose a new fuzzy reinforcement learning algorithm for differential games that have continuous state and action spaces. The proposed algorithm makes use of function approximation systems (fuzzy inference systems, FISs) and reinforcement learning methods. We derive the updating laws for the input and output parameters of the FISs of the proposed algorithm. Simulation results show that the proposed algorithm outperforms the state-of-the-art fuzzy 
reinforcement learning algorithms.

\section{Chapter 5 - A Fuzzy Reinforcement Learning Algorithm Using a Predictor for Pursuit-Evasion Games}

In this chapter, we propose a new fuzzy reinforcement learning algorithm for pursuit-evasion games. The proposed algorithm trains the pursuer so that the trained pursuer can capture the evader even when the environment of the game is different from the training environment. We evaluate the proposed algorithm on different pursuit-evasion games that have environments different from the training environment the pursuer was trained on. Simulation results are provided to show the practical performance of the proposed algorithm compared to the performances of the state-of-the-art fuzzy reinforcement learning algorithms.

\section{Chapter 6 - Decentralized Learning in Multi-Pursuer Pursuit-Evasion Dif- ferential Games with a Single Evader Having the Same Speed as the Pur- suers}

In this chapter, we propose a new decentralized fuzzy reinforcement learning algorithm for multi-pursuer pursuit-evasion differential games with a single-superior evader that has a speed similar to the speed of the pursuers. We present the formation control mechanism used by the proposed algorithm so that the superior evader can be enclosed by the pursuers so that they can capture it. We develop the reward function used by the proposed algorithm so that one or some of the learning pursuers can capture the superior evader. We also present the simulation results that show the effectiveness of the proposed algorithm. 
Chapter 7 - A Decentralized Fuzzy Learning Algorithm for Multi-Player Pursuit-Evasion Differential Games with a Single Evader Having a Speed Similar to or Higher Than the Speed of Pursuers

In this chapter, we propose a new decentralized fuzzy reinforcement learning algorithm for multi-pursuer pursuit-evasion differential games with a single-superior evader that has a speed similar to or higher than the speed of the pursuers. A new formation control mechanism is proposed in this work and is used with the Apollonius circle mechanism to construct the reward function of each learning pursuer. We also present the simulation results that show the performance of the proposed algorithm. 


\section{Chapter 2}

\section{Background and Preliminary Ideas}

\section{$2.1 \quad$ Introduction}

In this chapter, we briefly review some background information needed to formulate and solve reinforcement learning problems in discrete domains. Then, we briefly introduce some definitions and concepts in fuzzy systems needed to solve reinforcement learning problems in continuous domains.

\subsection{A Framework for Reinforcement Learning}

Reinforcement learning (RL) is a learning technique that maps situations to actions in order to maximize a reward signal [20]. In RL, an agent learns its strategy from the experience of interacting with its environment as shown in Fig 2.1. The figure consists of two blocks, an agent which takes actions and an environment which provides the agent with the corresponding states and rewards. At each time step, the learning agent perceives the state of its environment and then takes an action so that its environment transitions from the current state to a new state. A scalar reward signal is used to evaluate this transition. The objective for the learning agent is to maximize its cumulative reward $[18,20,114]$. To find which 


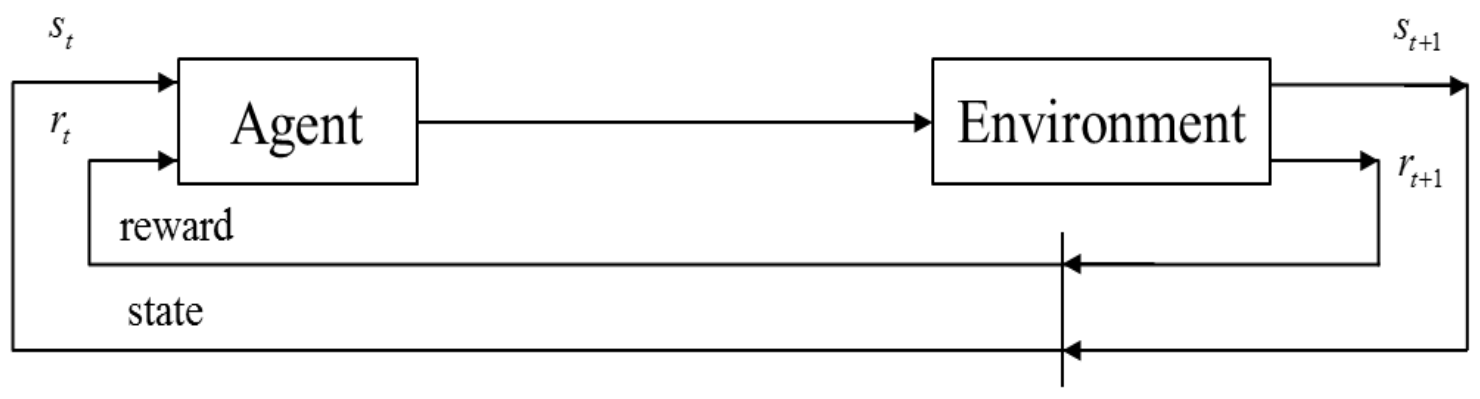

Figure 2.1: Agent-environment interaction in RL [20].

actions lead to the maximum cumulative reward, the learning agent must explore all its possible actions. Not only may the immediate rewards be affected by the actions of the learning agent but also the next situation and all subsequent rewards [20].

The main advantage of $\mathrm{RL}$ is that the learning agent does not require prior knowledge about the environment so as to learn its optimal policy; i.e. neither an expert nor a training data set is required by the agent to learn its optimal policies. The agent learns through the experience of interacting with the environment. All the agent requires to learn the optimal policies are the rewards and punishments.

\subsubsection{Markov Decision Processes}

A Markov Decision Process (MDP) is a well-known approach that is used to model single-agent environments. A single-agent environment is only represented by this model if the Markov assumption is justified. A MDP [5, 20] is defined as a tuple, $(S, A, T r, R)$, where,

- $S$ is the environment's finite set of states,

- $A$ is the agent's available finite set of actions,

- $\operatorname{Tr}$ is the transition function $S \times A \times S \rightarrow[0,1]$, where $\operatorname{Tr}(s, a, s)$ defines the 
environment's probability of transition to state $s \in S$ when the agent is at state $s \in S$ and takes action $a \in A$, and $\sum_{s \in S} \operatorname{Tr}\left(s, a, s^{\prime}\right)=1, \quad \forall s \in S$ and $\forall a \in A$,

- $\mathrm{R}$ is the reward function which defines the reward that the agent receives when the agent selects an action from the available actions at the given state and transitions to the next state.

An environment is called MDP (Markovian or stationary) if it satisfies the Markov property. That is, the next state of the environment is only a function of the current state and the agent's action taken at this particular state. This also means that the conditional probability distribution of the next state and reward of the player (agent) is only a function of the current state and action of the player such that

$$
\operatorname{Pr}\left\{s_{t+1}=\dot{s}, r_{t+1}=\dot{r} \mid s_{t}, a_{t}, \ldots, s_{0}, a_{0}\right\}=\operatorname{Pr}\left\{s_{t+1}=\dot{s}, r_{t+1}=\dot{r} \mid s_{t}, a_{t}\right\}
$$

MDPs are the basis for much of the research done in single agent control learning. A graphical definition of a Markov decision process is shown in Fig. 2.2. In a MDP, the objective of the agent is to find a policy $\pi: S \rightarrow A$ that maps states to actions so that the discounted future reward is maximized [20]. A player's policy $\pi$ at a given state $s$ is defined as a probability distribution over the player's actions at the given state, and it satisfies,

$$
\sum_{a \in A} \pi(s, a)=1 \quad \forall s \in S
$$

In $\mathrm{RL}$, the reward a learning agent receives from the interaction with its environment is a simple number $r_{t+1} \in R$. The sequence of rewards $\left(r_{t+1}, r_{t+2}, r_{t+3}, \ldots ..\right)$ the agent receives are used to formalize the agent's goal. Finding a policy that maximizes a discounted return, $\mathcal{R}_{t}$, is the goal of the learning agent. The discounted return, $\mathcal{R}_{t}$, 


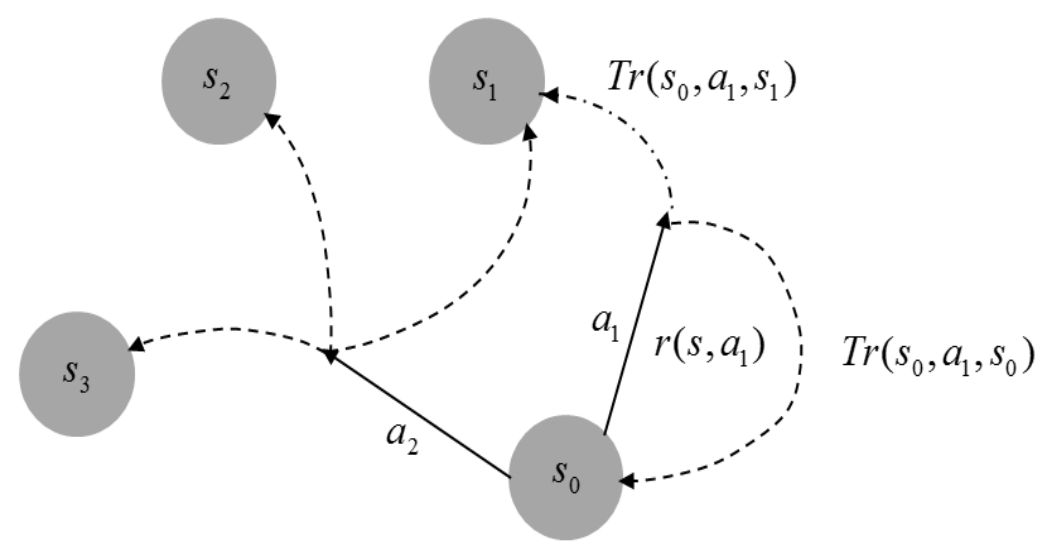

Figure 2.2: Graphical description of the Markov decision process (MDP) framework. Not all actions and transitions are shown.

is defined as follows,

$$
\mathcal{R}_{t}=\sum_{k=0}^{T_{f}-t-1} \zeta^{k} r_{t+k+1}
$$

where $t$ is the current time step, $r_{t+k+1}$ is the received immediate reward at the time step $t+k+1, \zeta \in(0,1]$ is a discount factor, and $T_{f}$ is the final time step in the learning episode. Each episode has an initial state that starts from and a terminal state where it ends at. The task that is finished in a finite time is called an episodic task. Similarly, the task that goes continually and has no limit is called a continuing task. Thus, for a continuing task, the final time $T_{f}$ in Eq. (2.3) is set as $T_{f} \rightarrow \infty$. When $\zeta=0$ in Eq. (2.3), maximizing the immediate reward becomes the only concern for the agent; the agent's goal, in this case, is to learn a strategy to maximize the immediate reward $r_{t+1}$. When $\zeta$ approaches 1 , the agent becomes more far-sighted and future rewards are taken into account by the agent more strongly [20].

To maximize a player's discounted return, the player's policy should be evaluated. To do so, the concept of the state-value function can be used. The state-value function 
under a policy $\pi$ is defined as the expected return when the player starts at state $s$ and follows a policy $\pi$ thereafter. That is,

$$
V^{\pi}(s)=E_{\pi}\left(\mathcal{R}_{t} \mid s_{t}=s\right)=E_{\pi}\left(\sum_{k=0}^{T_{f}-t-1} \zeta^{k} r_{k+t+1} \mid s_{t}=s\right),
$$

where $V^{\pi}(s)$ is the state-value function, and $E_{\pi}($.$) is the expected value under policy$ $\pi$.

The state-value function $V^{\pi}(s)$ of Eq. (2.4) can also be defined as follows [20], which is also known as Bellman equation,

$$
V^{\pi}(s)=\sum_{a \in A} \pi(s, a) \sum_{s \in S} \operatorname{Tr}(s, a, \dot{s})\left[r(s, a, \dot{s})+\zeta V^{\pi}(\hat{s})\right]
$$

where,

$$
\begin{gathered}
r(s, a, \dot{s})=E\left\{r_{t+1} \mid s_{t}=s, a_{t}=a, s_{t+1}=\dot{s}\right\}, \\
\operatorname{Tr}(s, a, \dot{s})=\operatorname{Pr}\left\{s_{t+1}=\dot{s} \mid s_{t}=s, a_{t}=a\right\},
\end{gathered}
$$

$r(s, a, s)$ is the expected immediate reward received at state $s$ given the current state $s$ and action $a$, and $\operatorname{Tr}(s, a, s)$ is the probability of the next state being $s_{t+1}$ given the current state $s_{t}=s$ and action $a_{t}=a$.

A player's policy can also be evaluated by using the concept of the action-value function. The action-value function $Q^{\pi}(s, a)$ (also known as the state-action value function) is defined as the expected return of selecting a particular action $a$ at a state $s$ and following a policy $\pi$ thereafter. The action-value function $Q^{\pi}(s, a)$ is given as 
follows [20],

$$
Q^{\pi}(s, a)=\sum_{s \in S} \operatorname{Tr}\left(s, a, s^{\prime}\right)\left[r\left(s, a, s^{\prime}\right)+\zeta V^{\pi}\left(s^{\prime}\right)\right]
$$

If the player follows an optimal policy $\pi^{*}$, the optimal state-value function $V^{*}(s)$ and the optimal action-value function $Q^{*}(s, a)$ can be defined as follows,

$$
\begin{gathered}
V^{*}(s)=\max _{a \in A} V^{\pi}(s), \\
Q^{*}(s, a)=\max _{\pi} Q^{\pi}(s, a) .
\end{gathered}
$$

The optimal state-value function $V^{*}(s)$ can also be calculated as follows [20],

$$
V^{*}(s)=\max _{a \in A} Q^{*}(s, a)
$$

Hence, the optimal policy $\pi^{*}(s)$ is given as follows,

$$
\pi^{*}(s)=\arg \max _{a \in A} Q^{*}(s, a)
$$

where $\arg \max _{a \in A} Q^{*}(s, a)$ denotes the action $a$ at which $Q^{*}(s, a)$ is maximized.

The optimal state-value function $V^{*}(s)$ and the optimal action-value function $Q^{*}(s, a)$ can be recursively calculated by using Dynamic Programming (DP) approaches. To use such approaches, the model of the environment has to be known in advance (.i.e the state transition probability $\operatorname{Tr}\left(s, a, s^{\prime}\right)$ and the reward function $r\left(s, a, s^{\prime}\right)$ have to be known). In real-world applications, however, the model of the environment is often unknown. Different RL methods such as model-free methods and model-based methods can be used instead to estimate the optimal state-value and action-value 
functions $[20,46,47]$.

\section{An example: Recycling Robot MDP [20]}

The recycling robot can provide a simple example of a MDP when it is simplified. It is important to mention here that this example is only to give some details about MDPs and does not represent a realistic recycling robot. Recall that the decision made by the recycling robot at each time step is based on either the external events or the other parts of the robot's control system. Three different decisions are available for the recycling robot so that it selects one each time step; wait for someone to bring it a can, search for a can, or return to its base to recharge its battery. The recycling robot's decisions are solely made based on its battery energy level. Two levels of energy can be distinguished, high and low. The state is therefore is set as $s=\{h i g h, l o w\}$. The recycling robot decisions (robot's actions) are assumed to be: search, wait and recharge. Searching for cans is assumed to be the best way to find them. However, the continuing search may deplete the robot's battery; if this happens, the robot would have to shut down and wait for a rescue. On the other hand, it is assumed that the robot's action "waiting" does not rundown the battery. Because recharging the battery when the energy level is high is not a good choice for the recycling robot, the robot's actions are then set as follows:

$$
\begin{gathered}
A(\text { high })=\{\text { search, wait }\}, \\
A(\text { low })=\{\text { search, wait, recharge }\} .
\end{gathered}
$$

Searching for cans with a high energy level in the recycling robot's battery can always be finished without the risk of depleting the battery. It is assumed that a period of searching for cans beginning with a high energy level ends with a high energy level in the robot's battery with a probability $p_{1}$ and with a low energy level 


\begin{tabular}{|l|l|l|l|l|}
\hline$s$ & $s$ & $a$ & $\operatorname{Pr}\left(s, a, s^{\prime}\right)$ & $r\left(s, a, s^{\prime}\right)$ \\
\hline high & high & search & $p_{1}$ & $R^{\text {search }}$ \\
\hline high & low & search & $1-p_{1}$ & $R^{\text {search }}$ \\
\hline high & high & wait & 1 & $R^{\text {wait }}$ \\
\hline high & low & wait & 0 & 0 \\
\hline low & high & search & $1-p_{2}$ & -3 \\
\hline low & low & search & $p_{2}$ & $R^{\text {search }}$ \\
\hline low & high & wait & 0 & 0 \\
\hline low & low & wait & 1 & $R^{\text {wait }}$ \\
\hline low & high & recharge & 1 & 0 \\
\hline low & low & recharge & 0 & 0 \\
\hline
\end{tabular}

Table 2.1: The expected rewards and transition probabilities for the finite MDP of the recycling robot example [20].

with a probability $1-p_{1}$. On the other hand, it is assumed that searching for cans when the energy level of the robot's battery is low ends with a low energy level in the robot's battery with a probability $p_{2}$ and with depleting the battery with a probability $1-p_{2}$. In the latter case, the robot must be returned to its base to recharge it back to high.

For simplicity, it is assumed that no cans can be collected when the recycling robot is returning home to its base for recharging. Let $R^{\text {search }}$ denotes the robot's reward during searching, and $R^{\text {wait }}$ denotes the robot's reward during waiting, where $R^{\text {search }}>R^{\text {wait }}$. The recycling robot is punished with -3 unit reward whenever its battery depleted and needs to be rescued. This system represents a MDP and its probabilities of transitions and expected rewards can be given as in Table 2.1. 


\subsubsection{Q-learning Algorithm}

The Q-learning algorithm $[24,25]$ is one of the most well-known algorithms in reinforcement learning. The Q-learning algorithm updates the long-term payoffs of state-action pairs by interacting with the environment. The Q-learning algorithm is a single-agent learning algorithm $[24,25]$ that can be used in MDPs to learn optimal policies. In single-agent learning, the Q-table of a learning agent is guaranteed to converge to optimal Q-values, and hence, the learning agent learns an optimal policy by selecting the greedy actions. The Q-learning table of agent $j$ is updated as follows,

$$
Q_{t+1}^{j}\left(s_{t}, a_{t}\right)=(1-\vartheta) Q_{t}^{j}\left(s_{t}, a_{t}\right)+\vartheta\left[r_{t}^{j}+\zeta \max _{a^{\prime}} Q_{t}^{j}\left(s_{t+1}, a^{\prime}\right)\right]
$$

where $Q_{t}^{j}\left(s_{t}, a_{t}\right)$ is the state-action value function of agent $j$ at the state $s_{t}$ and action $a_{t}, t$ is the number of times the state $s_{t}$ has been visited, $\vartheta$ is the learning rate, $r_{t}^{j}$ is the immediate reward of agent $j$ at the state $s_{t}, a_{t}$ is the action chosen by agent $j$ at the state $s_{t}, a^{\prime}$ is the action selected by agent $j$ at the next state $s_{t+1}$, and $\zeta$ is the discount factor.

The state-action value function (or the action-value function) $Q$ of Eq. (2.11) is guaranteed to converge to the optimal $Q^{*}$ if the following conditions are satisfied [20]:

(i) The state-action pair is visited an infinite number of times.

(ii) The learning rate $\vartheta$ is decaying over time provided that

$$
\sum_{t=0}^{\infty} \vartheta=\infty \text { and } \sum_{t=0}^{\infty} \vartheta^{2}<\infty
$$

Condition (i) states that each state-action pair has to be visited an infinite number of times which in turn emphasizes the importance of the exploration strategy. In this thesis, the $\epsilon$-greedy exploration policy is used. In this policy, the learning agent 
chooses the greedy action with a probability $1-\epsilon$ and chooses a random action with a probability $\epsilon$. Condition (i) is guaranteed to satisfy when $\epsilon>0$. Condition (ii), on the other hand, is a standard condition for stochastic approximation.

Although the Q-learning algorithm is a single-agent learning algorithm, it has been successfully used for multi-agent learning $[26,27,39]$. Despite the loss of theoretical guarantees, Q-learning agents often succeed to learn Nash equilibrium policies in multi-agent environment [23] because the Q-tables of the learning agents do not have to converge to optimal values in order for the agents to execute a Nash equilibrium policy, and the learning agents must adopt a Nash equilibrium if they are playing optimally.

\subsubsection{Matrix Games}

A matrix game (strategic game) $[33,34]$ can be described as a tuple $\left(\mathfrak{N}, A^{1, \ldots, \mathfrak{N}}, R^{1, \ldots, \mathfrak{N}}\right)$, where $\mathfrak{N}$ is the number of agents, $A^{j}$ is the discrete space of agent $j$ 's available actions, $R^{j}$ is agent $j$ 's payoff function, and $j=1, \ldots, \mathfrak{N}$. The learning agents receive payoffs that are functions of the actions selected by agents. The matrix games are called by this name because the payoff functions $R^{j}$ can be written as $\mathfrak{N}$-dimensional matrices. In matrix games, the objective of each agent is to find a pure or mixed strategy that maximizes its payoff. A pure strategy is the strategy that chooses actions deterministically, whereas a mixed strategy is the strategy that chooses actions based on a probability distribution over the agent's available actions.

\section{Types of Matrix Games}

Matrix games are often classified based on the structure of their payoff functions. One of the classes of these matrix games is called team games (fully cooperative 
games). In this class, the payoff functions of the learning agents are the same, such as in the coordination game shown in Fig. (2.3). Thus, the action in the best interest of one agent is in the best interest of all other agents. Zero-sum games (fully competitive games) are another class of matrix games. In this class, there are two learning agents; the reward of each agent is equal to the negative reward of the other agent. The matching pennies game is an examples of zero-sum matrix games. Some matrix games are neither team games nor zero-sum games, but they are referred to as general-sum games. All types of matrix games are sometimes referred to as generalsum games, although general-sum games are often meant to be the non-zero-sum games $[35,36]$.

\section{Nash Equilibrium in Matrix Games}

In a matrix game, each player is trying to follow a strategy so that its own reward is maximized. A player's strategy is the probability distribution over the available actions of the player. The concept of Nash equilibrium (NE) is addressed here to evaluate a player's strategy. A Nash equilibrium in a matrix game is a collection of all learning agents' strategies such that none of the learning agents can do better by changing its own strategy when the other learning agents are following their Nash strategies $[36,43,44]$. That is,

$$
\begin{gathered}
V^{j}\left(\pi^{1 *}, \ldots, \pi^{j *}, \ldots, \pi^{\mathfrak{N} *}\right) \geq V^{j}\left(\pi^{1 *}, \ldots, \pi^{j}, \ldots, \pi^{\mathfrak{N} *}\right), \\
\forall \pi^{j} \in \Pi^{j}, j=1, \ldots, \mathfrak{N}
\end{gathered}
$$

where $V^{j}($.$) is the value function of player j$ which represents the expected reward of player $j$ given all the other players' equilibrium strategies, and $\pi^{j}$ is any strategy of player $j$ from its strategy space $\Pi^{j}$. 
Matching Pennies Game

$$
R_{1,2}=\left[\begin{array}{cc}
1,-1 & -1,1 \\
-1,1 & 1,-1
\end{array}\right]
$$

$$
R_{1,2}=\left[\begin{array}{ll}
0,3 & 3,2 \\
1,0 & 2,1
\end{array}\right]
$$

$$
R_{1,2}=\left[\begin{array}{cc}
\text { Dilemma Game } \\
10,10 & 1,12 \\
12,1 & 2,2
\end{array}\right]
$$

$$
\begin{gathered}
\text { Shapley's Game } \\
R_{1,2}=\left[\begin{array}{ccc}
0,0 & 1,0 & 0,1 \\
0,1 & 0,0 & 1,0 \\
1,0 & 0,1 & 0,0
\end{array}\right] \quad R_{1,2}=\left[\begin{array}{cc}
1,1.85 & 1.85,1 \\
1.15,1 & 1,1.15
\end{array}\right]
\end{gathered}
$$

Figure 2.3: Matrix games.

\section{Examples}

Fig. 2.3 shows some examples of matrix games. The matching pennies game, tricky game, shapley's game, dilemma game, coordination game, and biased game are shown. In these games, the payoff of one player is represented by a row in the payoff matrix, and the payoff of the other player is represented by a column in the same payoff matrix. Each cell in the payoff matrix represents the payoff received by the row and column players, respectively. Both the matching pennies game and the tricky game have mixed Nash equilibrium strategies $(0.5,0.5)$. The shapley's game has a mixed Nash equilibrium strategy, $\left(\frac{1}{3}, \frac{1}{3}, \frac{1}{3}\right)$. The dilemma game has a pure Nash equilibrium strategy that executes the second action of each player with a probability of one. The coordination game has multiple pure Nash equilibrium strategies. On the other hand, the biased game has a mixed Nash equilibrium strategy with probabilities not uniform across actions, $(0.15,0.85)$ and $(0.85,0.15)$. 


\subsubsection{Stochastic Games}

A stochastic game can be described as a tuple $\left(\mathfrak{N}, S, A^{1, \ldots, \mathfrak{N}}, R^{1, \ldots, \mathfrak{N}}, \operatorname{Tr}\right)$ where $\mathfrak{N}$ is the number of agents, $S$ is the discrete space of states, $A^{j}$ is the discrete space of agent $j$ 's available actions, $R^{j}$ is the reward function, and $\operatorname{Tr}$ is the transition function [9]. A stochastic game can be described as an extension of a Markov decision process since the later contains a single player and multiple states. A stochastic game can also be described as an extension of a matrix game as the matrix game contains multiple players and a single state [9]. That is, each state in a stochastic game can be dealt with as a matrix game. Like MDPs, stochastic games also have the Markov property. That is, the next state and reward of the learning agent are only functions of the current state and the agent's available actions.

\section{Types of Stochastic Games}

Stochastic games have the same classification that matrix games have; team games (fully cooperative games), zero-sum games (fully competitive games), and generalsum games. In general, the general-sum stochastic games can be referred to all types of stochastic games, although they are usually meant to be non-zero-sum stochastic games. When all learning agents of a stochastic game have the same reward functions, the stochastic game is called a team (or fully cooperative) game. In two-player stochastic games, a stochastic game is called a zero-sum stochastic game if the reward function of the first player is equal to the negative of the reward function of the second player [35].

\section{Nash Equilibrium in Stochastic Games}

In a multi-player stochastic game and for all $s \in S$ and $j=1, \ldots, \mathfrak{N}$, a Nash equilibrium can be defined as a tuple of $\mathfrak{N}$ strategies $\left(\pi^{1 *}, \ldots, \pi^{\mathfrak{N} *}\right)$ such that 


$$
\begin{gathered}
V^{j}\left(s, \pi^{1 *}, \ldots, \pi^{j *}, \ldots, \pi^{\mathfrak{N} *}\right) \geq V^{j}\left(s, \pi^{1 *}, \ldots, \pi^{j}, \ldots, \pi^{\mathfrak{N} *}\right), \\
\forall \pi^{j} \in \Pi^{j}, j=1, \ldots, \mathfrak{N},
\end{gathered}
$$

where $V^{j}\left(s, \pi^{1 *}, \ldots, \pi^{\mathfrak{N} *}\right)$ is the value function of player $j$ which represents the expected reward of player $j$ given all the other players' equilibrium strategies at the current state, and $\pi^{j}$ is any strategy of player $j$ 's strategy space $\Pi^{j}$.

\section{Examples}

Fig. 2.4 shows two stochastic games introduced by $\mathrm{Hu}$ and Wellman [16]. The players in both games are located in the lower corners and are allowed to move one cell in the four compass directions (North, East, South and West). The transition is ignored if both players move to the same cell (excluding a goal cell).The players' goals in both grid games are located as shown in Fig. 2.4. The transition in grid game 1 is deterministic; grid game 2, on the other hand, has deterministic and probabilistic transitions. At the lower corners in grid game 2, the probability of transition to the next cell is 0.5 when the player takes the action North. In both grid games, the player that reaches its goal is rewarded 100 points, it receives -1 points when either it hits the wall or moves into the cell that the other player moves into (excluding a goal cell), and it receives 0 points otherwise. Reaching its goal with a minimum number of steps (transitions) is therefore the aim of each player in both games. As soon as a player reaches its goal, the game ends [16]. Grid game 1 has ten different Nash equilibrium strategies; whereas grid game 2 has two different Nash equilibrium strategies [16]. Fig. 2.5 shows one Nash equilibrium for each grid game. 


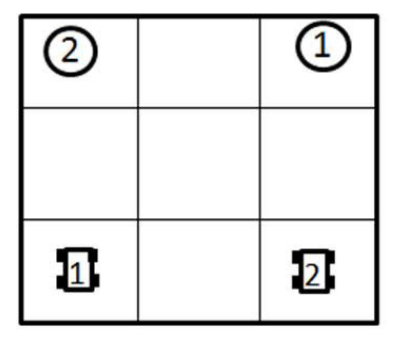

(a) Grid Game 1

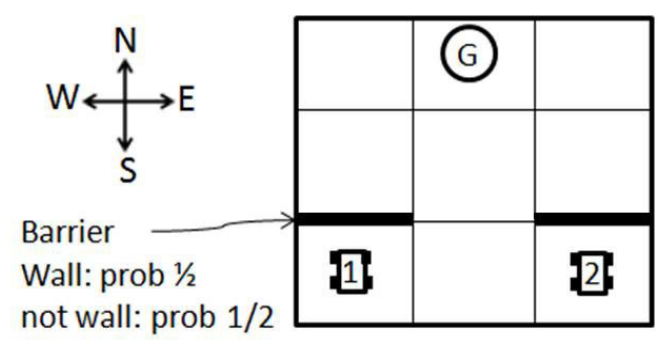

(b) Grid game 2

Figure 2.4: Two stochastic games [16].

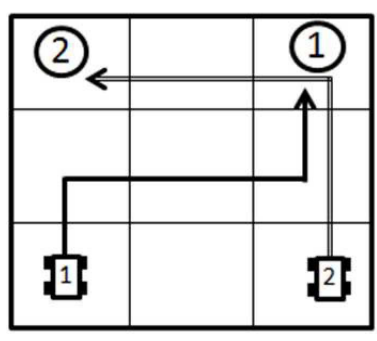

(a)

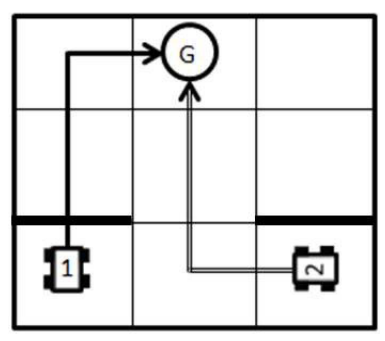

(b)

Figure 2.5: Selected Nash equilibrium paths of grid game 1 [16]. 


\section{$2.3 \quad$ Fuzzy Systems}

Fuzzy systems have been widely used in a variety of applications in many different fields such as engineering, business, medicine and psychology [143]. In engineering, for example, fuzzy systems have been applied to different application areas such as [143]:

- process control: temperature control, pressure control, and level control;

- automobiles: engine control, transmission control, and brakes control;

- automated highway systems: cruise control;

- autonomous vehicles: ground and underwater control;

- aircraft/spacecraft: flight control and engine control;

- power industry: power and motor control;

- manufacturing systems: decomposition process control and scheduling control.

Fuzzy systems are also known by a number of names such as fuzzy inference systems (FISs), fuzzy logic controllers (FLCs), fuzzy expert systems, and fuzzy models. Fig. (2.6) shows a block diagram of a fuzzy control system, where the fuzzy controller (fuzzy logic controller, FLC) is embedded in a closed-loop control system. A fuzzy inference system is composed of four main elements [141,143]:

- a rule base: a set of IF-THEN rules that hold the expert's knowledge of how to achieve the best control to get the system's desired performance;

- an inference engine: it is also called an inference mechanism and it applies the appropriate control value to the plant (system) based on the evaluation of which control rules are relevant at the current time; 


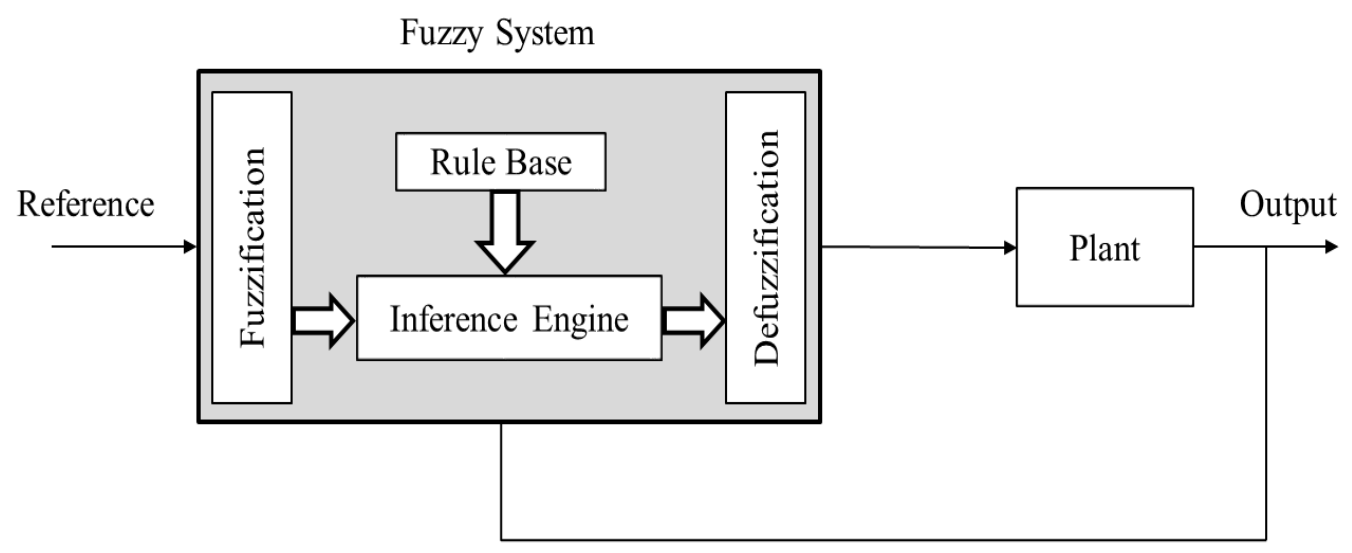

Figure 2.6: Fuzzy control system architecture [143].

- a fuzzification interface: it converts the controller inputs into information so that they can be interpreted and used by the rule-base unit;

- a defuzzification interface: it converts the conclusions received from the inference engine into control values to the plant.

There are several types of FISs presented in the literature [140-143]. The Mamdani FIS proposed in [145] and the Takagi-Sugeno-Kang (TSK) FIS proposed in [146,147] are the most popular ones [140-143].

\subsubsection{Membership Function}

A membership function (MF) of a fuzzy set is a continuous function that takes values in the interval $[0,1]$ and it characterizes the fuzzy set in a universe of discourse $\mathcal{S}$ $[140,141]$. The universe of discourse $\mathcal{S}$ is defined as a collection of elements $s$ that have the same characteristics. A fuzzy set in a universe of discourse $\mathcal{S}$ is defined as follows $[140,141]$,

$$
\mathcal{A}=\left\{\left(s, \mu_{\mathcal{A}}(s)\right) \mid s \in \mathcal{S}\right\}
$$


where $\mu_{\mathcal{A}}(s)$ is the MF of the fuzzy set $\mathcal{A}$. There are different types of MFs, where each type is defined with a different number of coefficients. For example, Gaussian MF is defined with two coefficients; whereas the triangular MF is defined with three coefficients, and the trapezoidal MF is defined with four coefficients. In real-time implementation and control, Gaussian and trapezoidal MFs are often used. A Gaussian MF can be defined as follows,

$$
\mu_{\mathcal{A}}(s)=\exp \left(-\frac{1}{2}\left(\frac{s-m}{\sigma}\right)^{2}\right)
$$

where $m$ is the mean and $\sigma$ is the standard deviation. Fig (2.7) shows a Gaussian MF. The figure shows that the Gaussian MF is not linear but smooth.

A trapezoidal MF, on the other hand, can be defined as follows,

$$
\mu_{\mathcal{A}}(s)=\left\{\begin{aligned}
0 & : \quad s<a, \\
\frac{s-a}{b-a} & : \quad a \leq s<b, \\
1 & : \quad b \leq s \leq c, \\
\frac{s-c}{d-c} & : \quad c<s \leq d, \\
0 & : \quad s>d,
\end{aligned}\right.
$$

where $a, b, c$, and $d$ are the MF's parameters. Fig. (2.8) shows a trapezoidal MF. Unlike the Gaussian MF, the trapezoidal MF is linear but not smooth. 


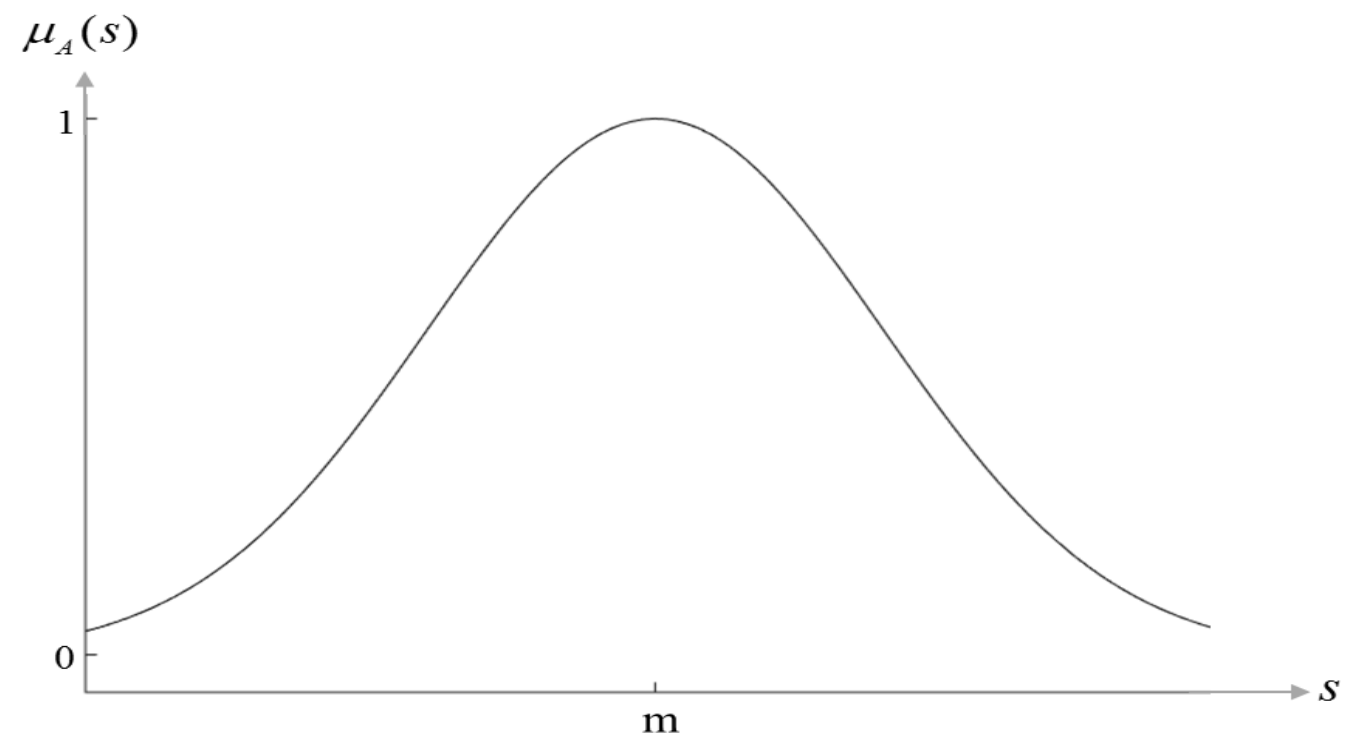

Figure 2.7: A Gaussian MF.

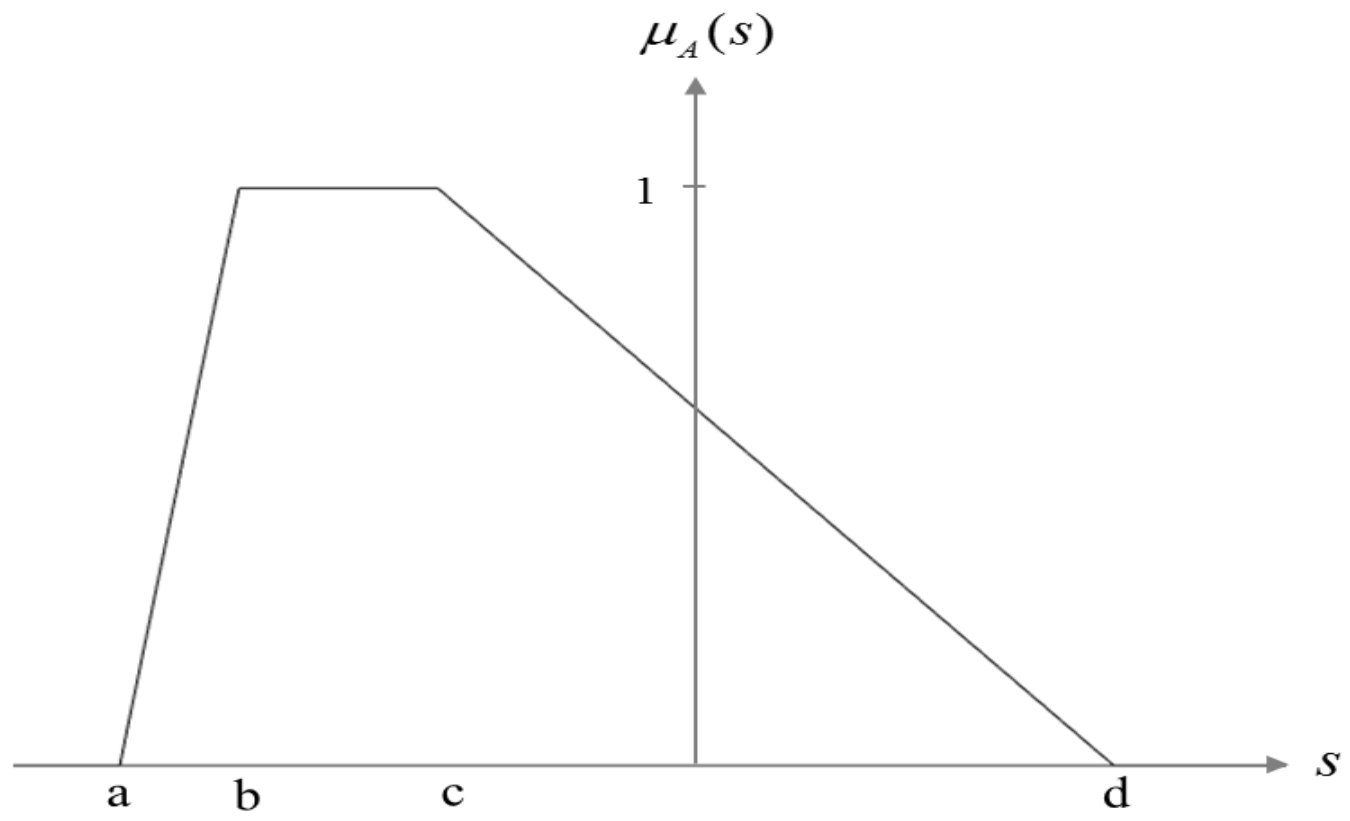

Figure 2.8: A trapezoidal MF. 


\subsubsection{Fuzzy IF-THEN Rules}

Fuzzy IF-THEN rules represent the human knowledge in an environment of uncertainty and imprecision [140]. For example, the Fuzzy IF-THEN rules used in Mamdani fuzzy inference system (FIS) are defined as follows [140,141],

$$
R_{l}: \text { IF } s_{1} \text { is } \mathcal{A}_{1}^{l}, \ldots, \text { and } s_{n} \text { is } \mathcal{A}_{n}^{l} \quad \text { THEN } z_{l}=\mathcal{B}^{l}, \omega_{l}=q_{l} \text {, }
$$

where $R_{l}$ is the rule $l, l=1, \ldots, L$ and $L$ is the number of rules in the fuzzy rule-base. The term $\mathbf{s}=\left(s_{1}, \ldots, s_{n}\right)^{T}$ represents the vector of the input linguistic variables, and $n$ is the number of input variables. The variable $z_{l}$ represents the output linguistic variable of the rule $l$. On the other hand, $\mathcal{A}_{\imath}^{l}$ and $\mathcal{B}^{l}$ are fuzzy sets defined in the universes of discourse $\mathcal{S}_{\imath}$ and $\mathcal{Z}$, respectively, where $\imath=1, \ldots, n$. The term $\omega_{l}$ represents the weight of the rule $R_{l}$ and $q_{l} \in[0,1]$. The first part of the fuzzy IF-THEN rule is called the antecedent (or premise) of the rule; whereas the second part of the IF-THEN rule is called the conclusion (or consequence) part of the rule.

On the other hand, the fuzzy IF-THEN rules used in Takagi-Sugeno-Kang (TSK) FIS are given as follows,

$$
R_{l}: \text { IF } s_{1} \text { is } \mathcal{A}_{1}^{l}, \ldots \text {, and } s_{n} \text { is } \mathcal{A}_{n}^{l} \quad \text { THEN } z_{l}={ }^{0} k_{l}+\ldots+{ }^{n} k_{l} s_{n} \text {, }
$$

where $z_{l}$ is the output function of the rule $l$, and ${ }^{0} k_{l}$ to ${ }^{n} k_{l}$ are the consequent parameters of the rule $l$. When $z_{l}$ is constant, the TSK FIS is called a zero-roder TSK FIS; whereas a first order TSK FIS means that the output, $z_{l}$, is a linear function of its inputs $[140,141,143]$. 


\subsubsection{Fuzzy Inference Engine}

A fuzzy inference engine combines the fuzzy IF-THEN rules in the fuzzy rule base into a mapping from a fuzzy set $\mathcal{A}^{\prime}$ in $\mathcal{S}$ to a fuzzy set $\mathcal{B}^{\prime}$ in $\mathcal{Z}$. A practical fuzzy rule base often has more than one rule. Two ways can be used to infer with a set of rules: composition based inference and individual-rule based inference.

\section{Composition Based Inference}

A composition based inference combines all rules in the fuzzy rule base into a single fuzzy relation in $\mathcal{S} \times \mathcal{Z}$. This single fuzzy relation is then viewed as a single fuzzy

IF-THEN rule [141]. A set of rules can be viewed as either independent conditional statements or strongly coupled conditional statements. When they are viewed as independent conditional statements, a reasonable operator for combining the rules is union. On the other hand, when they are viewed as strongly coupled conditional statements, the operator intersection should be used to combine the rules [141].

\section{Individual-Rule Based Inference}

In this inference, each rule in the fuzzy rule-base determines an output fuzzy set. The output of the whole individual-rule based inference is determined by the combination of the output fuzzy set of all rules [141].

There are a variety of choices in fuzzy reference engines. The product inference engine and the minimum inference engine are the most commonly used fuzzy inference engines in fuzzy systems [141]. 


\section{The Product Inference Engine}

A product inference engine uses individual rule based inference with union combination, Mamdani's product implication, and algebraic product for all t-norm operators and $\max$ for all s-norm operators. For the IF-THEN rule given in Eq. (2.17), the output of a product inference engine is defined as follows [141-143],

$$
\mu_{\mathcal{B}^{\prime}}(z)=\max _{l=1}^{L}\left[\sup _{\mathbf{s} \in \mathcal{S}}\left(\mu_{\mathcal{A}^{\prime}}(\mathbf{s}) \prod_{\imath=1}^{n} \mu_{\mathcal{A}_{\imath}^{l}}\left(s_{\imath}\right) \mu_{\mathcal{B}^{l}}(z)\right)\right] .
$$

\section{The Minimum Inference Engine}

A minimum inference engine uses individual-rule based inference with union combination, Mamdani's minimum implication, and min for all t-norm operators and max for all s-norm operators. For the IF-THEN rule given in Eq. (2.17), the output of a minimum inference engine is defined as follows [141-143],

$$
\mu_{\mathcal{B}^{\prime}}(z)=\max _{l=1}^{L}\left[\sup _{\mathbf{s} \in \mathcal{S}} \min \left(\mu_{\mathcal{A}^{\prime}}(\mathbf{s}), \mu_{\mathcal{A}_{1}^{l}}\left(s_{1}\right), \ldots, \mu_{\mathcal{A}_{n}^{l}}\left(s_{n}\right), \mu_{\mathcal{B}^{l}}(z)\right)\right]
$$

\subsubsection{Fuzzification and Defuzzification}

The fuzzifier is the first block of the fuzzy system shown in Fig. (2.6). The fuzzifier converts each crisp input variable to degrees of membership by a lookup in one or several membership functions [144]. Thus, fuzzification is the process of making a crisp quantity fuzzy so that it can be used by the fuzzy system $[142,143]$. On the other hand, defuzzification is an opposite process to fuzzification as it converts a fuzzy quantity to a precise quantity [142]. There are several defuzzification methods that have been presented in the literature [140-143] such as Max membership principle (height method), Centroid method (center of area or center of gravity), Mean max membership (middle-of-maxima), and Weighted average (Center average) method. The Center of area method (COA) is a popular defuzzification method used 
with Mamdani FIS; the Weighted average (Center average) method is also a popular defuzzification method and it is used with Takagi-Sugeno-Kang (TSK) FIS. The Weighted average (Center average) method is defined as follows [142],

$$
Z(\mathbf{s})=\frac{\sum_{l=1}^{L}\left[\left(\prod_{\imath=1}^{n} \mu^{\mathcal{A}_{\imath}^{l}}\left(s_{\imath}\right)\right) z_{l}\right]}{\sum_{l=1}^{L}\left(\prod_{\imath=1}^{n} \mu^{\mathcal{A}_{\imath}^{l}}\left(s_{\imath}\right)\right)} .
$$

\subsection{Summary and Discussion}

In this chapter, we provided background information on reinforcement learning and fuzzy systems. We presented some introductory concepts which form the fundamental background for the work presented in the subsequent chapters. In reinforcement learning, we presented MDPs and gave an example that illustrates the graphical description of a MDP. We also presented the well-known Q-learning algorithm. In addition, we presented the basic concepts and definitions of matrix games. We illustrated the types of matrix games and the concepts of Nash equilibrium in matrix games. Moreover, we presented the basic concepts of stochastic games. We demonstrated the types of stochastic games and the concepts of Nash equilibrium in stochastic games. We presented two grid games as examples for stochastic games. In fuzzy systems, on the other hand, we introduced the concepts of the membership functions and presented the fuzzy IF-THEN rules. In addition, we introduced the main four elements that a fuzzy system consists of; rule base (fuzzy IF-THEN rules), fuzzy inference engine, fuzzifier and defuzzifier. In fuzzy inference engine, we presented the two different ways that can be used to infer with a set of rules; composition based inference and individual-rule based inference. We also introduced the two most commonly used fuzzy inference engines in fuzzy systems; the product inference engine and the minimum inference engine. 


\section{Chapter 3}

\section{Multi-agent Reinforcement Learning in Matrix and Stochastic Games}

\subsection{Introduction}

Reinforcement learning is well-suited for multi-agent learning because of its simplicity and generality $[10,11,15]$. Learning is a key element of multi-agent systems (MAS) as it allows each agent to improve its performance by adapting to the dynamics of the other agents and the environment [22]. Learning encounters some challenges when it is used in multi-agent learning. One of these challenges is that the other learning agents have to be explicitly considered by each learning agent and therefore the environment is non-stationary. The environment of a multi-agent system is no longer stationary as the Markov property is violated by the other learning agents. As a result of a non-stationary environment, single agent reinforcement learning techniques are not guaranteed to converge in multi-agent settings. In multi-agent learning, the objective of each learning agent is to adopt an equilibrium strategy that maximizes its payoffs in the long run. However, a globally optimal equilibrium may not be reached in some cases when the learning agents do not cooperate with each other $[1,39]$. The objective of each learning agent, in such cases, is to adopt 
a Nash equilibrium (NE) strategy, where the learning agent will not do better if it deviates from Nash equilibrium $[1,8,14,40]$.

In this chapter, we consider multi-agent domains in which different agents with different independent goals and assumptions have to interact with each other. We are interested in multi-agent learning algorithms that make agents learn how to adapt to changes in the other agents' performance when the Markovian property is no longer valid. That is, we are interested in multi-agent learning algorithms that can make agents learn Nash equilibrium strategies in a difficult learning problem with a moving target. Several multi-agent reinforcement learning (MARL) algorithms have recently been proposed and studied [1, 8, 9, 14, 16, 19, 22, 40-42]. All these algorithms assume that each learning agent knows its own immediate reward and the actions of the other learning agents. Some of these algorithms have theoretical results of convergence in general-sum games. In addition, some of these algorithms fail to converge to Nash equilibrium in some games. For example, the Infinitesimal Gradient Ascent (IGA) algorithm proposed in [19] fails to converge in games with mixed Nash equilibrium. The Win-or-Learn-Fast Infinitesimal Gradient Ascent (WoLF-IGA) algorithm proposed in [41] and the Win-or-Learn-Fast Generalized Infinitesimal Gradient Ascent (GIGA-WoLF) algorithm proposed in [8] fail to converge in some challenging games such as in the Shapley's game [1]. The Win-or-Learn-Fast Policy Hill-Climbing (WoLF-PHC) algorithm proposed in [9] does not converge to Nash equilibrium in the Shapley's game. In addition, some of these algorithms guarantee converge to Nash equilibrium by making some strict assumptions on the knowledge that is available to each learning agent. For example, some algorithms assume that the underlying game structure (Nash equilibrium) is known to each learning agent $[9,40]$. Other algorithms such as the algorithms proposed in $[14,16]$ assume that each learning agent knows the actions and the 
immediate rewards of the other learning agents [1,22]. Such strict assumptions may limit the use of these algorithms because the underlying game structure (Nash equilibrium) and the rewards of the other learning agents are often unknown to the learning agent and may be learned via interacting with the other learning agents [9]. On the other hand, the Weighted Policy Learner (WPL) and the Policy Gradient Ascent with Approximate Policy Prediction (PGA-APP) algorithms proposed in $[1,22]$ empirically converge to Nash equilibrium in a wider variety of situations without requiring the knowledge of the other agents' immediate rewards or strategies.

In this chapter, we are interested in proposing a multi-agent learning algorithm that can converge to Nash equilibrium in a wider variety of situations. In addition, we are interested in proposing a multi-agent learning algorithm that does not make strict assumptions that are often unknown and need to be learned via experience. In this chapter, we propose two MARL algorithms. The algorithms proposed in this chapter have already been published in [2-4]. The first proposed algorithm can successfully converge to Nash equilibrium policies in games that have pure Nash equilibrium. The second proposed algorithm, on the other hand, can successfully learn Nash equilibrium policies in games that have pure or mixed Nash strategies. The proposed algorithms use the exponential moving average (EMA) approach in parallel with the greedy action of the learning agent's Q-table as a basis to update the learning agent's strategy. We evaluate the proposed algorithms on a variety of matrix and stochastic games. The results show that the second proposed algorithm outperforms the state-of-the-art multi-agent learning algorithms in terms of convergence to Nash equilibrium. 


\subsection{An Overview on Some Multi-agent Reinforce- ment Learning Algorithms}

In this section, we present three different MARL algorithms. These three MARL algo-

rithms are: the Policy Gradient Ascent with Approximate Policy Prediction (PGAAPP) algorithm, the Weighted Policy Learner (WPL) algorithm, and the Win-orLearn-Fast Policy-Hill Climbing (WoLF-PHC) algorithm. We will compare the performance of the proposed algorithms with the performance of those three algorithms.

\subsubsection{The Policy Gradient Ascent with Approximate Policy Prediction (PGA-APP) Algorithm}

The Policy Gradient Ascent with Approximate Policy Prediction (PGA-APP) algorithm [22] is a practical multi-agent learning algorithm. This multi-agent PGA-APP algorithm extends the Q-learning algorithm and is capable of learning stochastic strategies. The key idea behind this algorithm is that the learning agent adjusts its strategy in response to forecasted strategies of the other agents, instead of their current ones. The PGA-APP algorithm is a decentralized algorithm as it only requires the observation of the reward of the learning agent received when the learning agent selects an action. The PGA-APP algorithm uses the policy gradient technique associated with the idea of policy prediction as a mechanism to learn stochastic strategy. The PGA-APP uses the Q-learning algorithm to learn the expected value of the learning agent at the state-action pair $\left(s_{t}, a\right)$. The expected value $Q\left(s_{t}, a\right)$ and the policy $\pi\left(s_{t}, a\right)$ at the state-action pair $\left(s_{t}, a\right)$ is used by the PGA-APP algorithm to estimate the partial derivative of the policy of the learning agent with respect to the current strategies at this state-action pair. The PGA-APP algorithm uses the idea of policy prediction to approximate the partial derivative of the policy of the other 
learning agent.

$\overline{\text { Algorithm } 1 \text { the policy gradient ascent with approximate policy prediction (PGA- }}$ APP) algorithm for agent $j$.

\section{1: Initialize:}

the learning rate $\vartheta$ and the discount factor $\zeta$ of the Q-learning.

the learning rate $\eta^{P}$ and the derivative prediction length $\gamma^{P}$ of the PGA-APP algorithm.

2: Initialize: $Q^{j}(s, a) \leftarrow 0$ and $\pi^{j}(s) \leftarrow I C s$

\section{3: Repeat}

4: From the state $s_{t}$, select an action $a_{t}$ according to the strategy $\pi_{t}^{j}\left(s_{t}\right)$ with some exploration.

5: Observe the immediate reward $r_{t}^{j}$, the new state $s_{t+1}$, and update $Q_{t+1}^{j}\left(s_{t}, a_{t}\right)$ using Eq. (2.11)

6: Update average reward $V^{j}\left(s_{t}\right)$

7: foreach action $a \in A$ do

update $\pi_{t+1}^{j}\left(s_{t}, a\right)$ based on Eq. (3.1)

\section{end}

8: $\pi_{t+1}^{j}\left(s_{t}\right)=\operatorname{limit}\left(\pi_{t+1}^{j}\left(s_{t}\right)\right)$

9: until the process is terminated.

The agent $j$ learning its strategy by the PGA-APP algorithm updates its Q-table as in Eq. (2.11), and updates its policy as follows [22],

$$
\begin{gathered}
\pi_{t+1}^{j}\left(s_{t}, a\right)=\pi_{t}^{j}\left(s_{t}, a\right)+\eta^{P} \delta\left(s_{t}, a\right), \\
\pi_{t+1}^{j}\left(s_{t}\right)=\operatorname{limit}\left(\pi_{t+1}^{j}\left(s_{t}\right)\right),
\end{gathered}
$$

where, 


$$
\delta\left(s_{t}, a\right)=\hat{\delta}\left(s_{t}, a\right)-\gamma^{P}\left|\hat{\delta}\left(s_{t}, a\right)\right| \pi_{t}^{j}\left(s_{t}, a\right)
$$

The term $\hat{\delta}\left(s_{t}, a\right)$ is given as follows,

$$
\hat{\delta}\left(s_{t}, a\right)= \begin{cases}Q_{t}^{j}\left(s_{t}, a\right)-V^{j}\left(s_{t}\right) & \text { if } \pi_{t}^{j}\left(s_{t}, a\right)=1 \\ \left.\left(Q_{t}^{j}\left(s_{t}, a\right)-V^{j}\left(s_{t}\right)\right) /\left(1-\pi_{t}^{j}\left(s_{t}, a\right)\right)\right) & \text { otherwise }\end{cases}
$$

and,

$$
\begin{aligned}
V^{j}\left(s_{t}\right) & =\sum_{a \in A} \pi_{t}^{j}\left(s_{t}, a\right) Q_{t}^{j}\left(s_{t}, a\right), \\
\operatorname{limit}(\pi) & =\operatorname{argmin}_{x: \operatorname{valid}(x)}|\pi-x|
\end{aligned}
$$

where $\operatorname{argmin}_{x: \operatorname{valid}(x)}|\pi-x|$ denotes the value of $x$ at which $|\pi-x|$ is minimized provided that $\sum x=1$.

The term $\pi_{t+1}^{j}\left(s_{t}\right)$ represents the policy vector at the state $s_{t}$, the term $\pi_{t+1}^{j}\left(s_{t}, a\right)$ represents the policy element at the state-action pair $\left(s_{t}, a\right)$, and $V^{j}\left(s_{t}\right)$ represents the average reward (or the state value function) at the state $s_{t}$. The term $\delta\left(s_{t}, a\right)$ represents the policy gradient, and the term $\hat{\delta}\left(s_{t}, a\right)$ represents the partial derivative of the expected payoff of the learning agent with respect to its current strategy. $\eta^{P}$ is a learning rate, and $\gamma^{P}$ is a small constant that represents the derivative prediction length. Algorithm 1 lists the procedure of the PGA-APP algorithm for the learning player $j$.

\subsubsection{The Weighted Policy Learner Algorithm}

The weighted policy learning WPL algorithm [1] uses the policy gradient mechanism to update the policy of the learning agent. The WPL algorithm assumes that the value function of the game is known to all players, which is then used to calculate the policy gradient $\delta\left(s_{t}, a\right)$. The WPL algorithm was shown to converge 
to Nash equilibrium in benchmark two-player-two-action games as well as some larger games. The WPL algorithm only requires the least information to converge to Nash equilibrium; it only requires the reward that the learning agent receives when it selects its action. The WPL algorithm does not require the knowledge of the other agents' actions nor rewards. In addition, it does not require the knowledge of the underlying game or the corresponding Nash equilibrium a priori. The learning mechanism of the WPL algorithm is to learn fast when the policy gradient changes its direction and to learn cautiously when the policy gradient does not change its direction. The direction of the policy gradient is detected by $\hat{\delta}\left(s_{t}, a\right)$, which is the difference between the expected value function $Q\left(s_{t}, a\right)$ and the average reward $V\left(s_{t}\right)$.

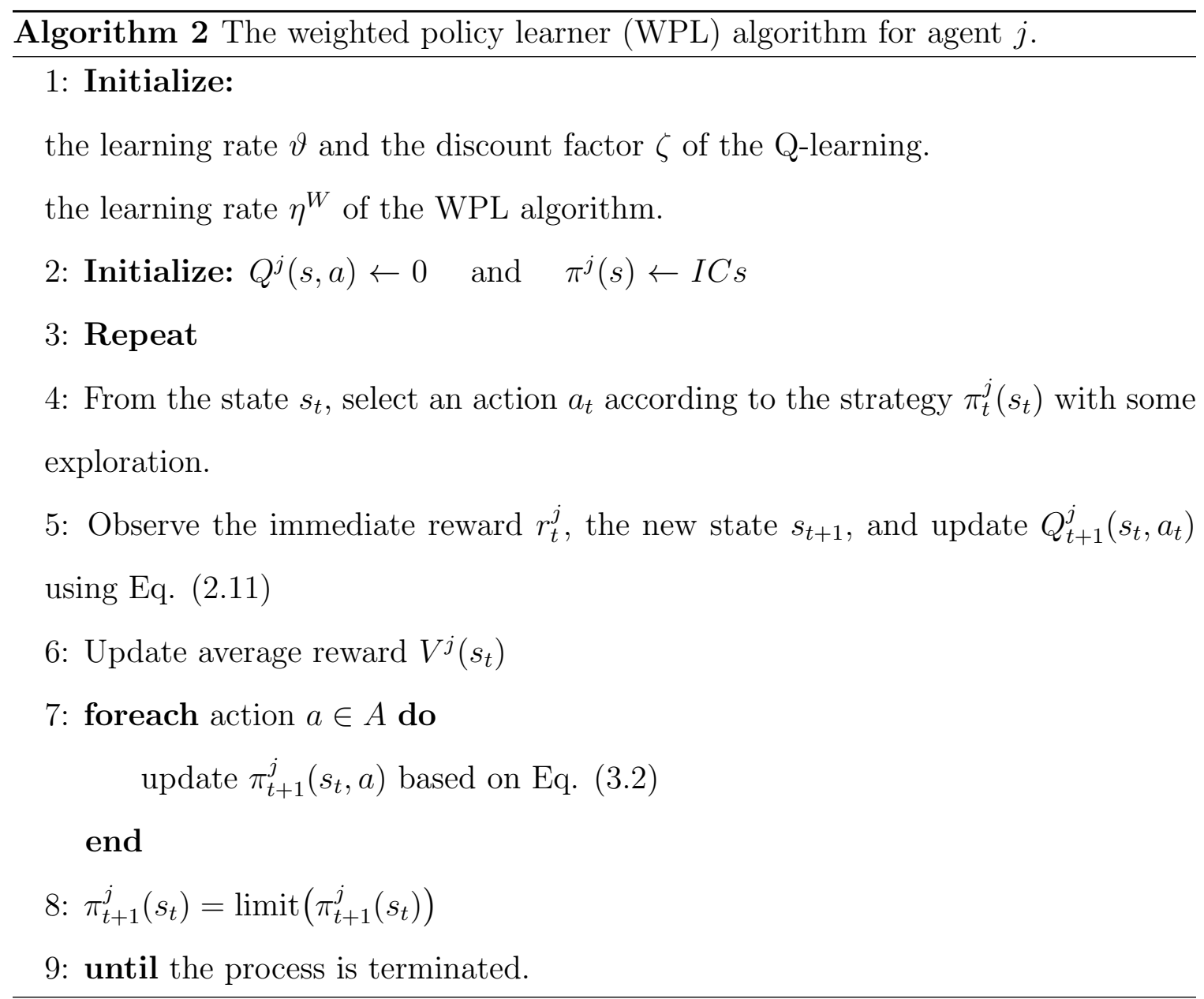


The agent $j$ learning its strategy by the WPL algorithm updates its Q-table as in Eq. (2.11), and updates its policy as follows [1],

$$
\pi_{t+1}^{j}\left(s_{t}, a\right)=\pi_{t}^{j}\left(s_{t}, a\right)+\eta^{W} \delta\left(s_{t}, a\right)
$$

and,

$$
\pi_{t}^{j}\left(s_{t+1}\right)=\operatorname{limit}\left(\pi_{t}^{j}\left(s_{t+1}\right)\right)
$$

where,

$$
\begin{aligned}
& \delta\left(s_{t}, a\right)= \begin{cases}\hat{\delta}\left(s_{t}, a\right)\left(1-\pi_{t}^{j}\left(s_{t}, a\right)\right) & \text { if } \hat{\delta}\left(s_{t}, a\right)>0, \\
\hat{\delta}\left(s_{t}, a\right)\left(\pi_{t}^{j}\left(s_{t}, a\right)\right) & \text { otherwise, }\end{cases} \\
& \hat{\delta}\left(s_{t}, a\right)=Q_{t}^{j}\left(s_{t}, a\right)-V^{j}\left(s_{t}\right), \\
& V^{j}\left(s_{t}\right)=\sum_{a \in A} \pi_{t}^{j}\left(s_{t}, a\right) Q_{t}^{j}\left(s_{t}, a\right), \\
& \operatorname{limit}(\pi)=\operatorname{argmin}_{x: \operatorname{valid}(x)}|\pi-x| .
\end{aligned}
$$

The term $\pi_{t+1}^{j}\left(s_{t}\right)$ is the policy vector at the state $s_{t}$, and the term $\pi_{t+1}^{j}\left(s_{t}, a\right)$ is the policy element at the state-action pair $\left(s_{t}, a\right)$. The term $\delta\left(s_{t}, a\right)$ represents the policy gradient, and the term $\hat{\delta}\left(s_{t}, a\right)$ represents the difference between the expected value function $Q_{t}^{j}\left(s_{t}, a\right)$ and the average reward $V^{j}\left(s_{t}\right) . \eta^{W}$ is a learning rate. Algorithm 2 shows the procedure of the WPL algorithm for the learning player $j$.

\subsubsection{Win or Learn Fast Policy Hill-Climbing (WoLF-PHC) Algorithm}

The win-or-learn-fast policy hill-climbing (WoLF-PHC) algorithm is an extension of the policy hill-climbing (PHC) algorithm [9]. This algorithm uses the Win-orLearn-Fast (WoLF) mechanism so that the PHC algorithm converges to a Nash equilibrium in self-play (the learning agents use the same type of algorithms to 
learn their strategies). The algorithm has two different learning rates, $\delta_{w}$ when the algorithm is winning and $\delta_{l}$ when it is losing. The WoLF-PHC algorithm uses the difference between the average strategy-based expected return and the current strategy-expected return as a criterion to decide when the algorithm wins or loses. The learning rate $\delta_{l}$ is bigger than the learning rate $\delta_{w}$. As such, the algorithm learns fast when it is losing. This causes the agent to adapt quickly to the changes in the strategies of the other agents when it is doing more poorly than expected and learns cautiously when it is doing better than expected [9]. This also gives the other agents the time to adapt to the agent's strategy changes. The WoLF-PHC algorithm exhibits the property of convergence as it makes the agent converge to one of its Nash equilibrium. This permits the WoLF-PHC algorithm to be widely applied to a variety of stochastic games $[9,10,37,38]$.

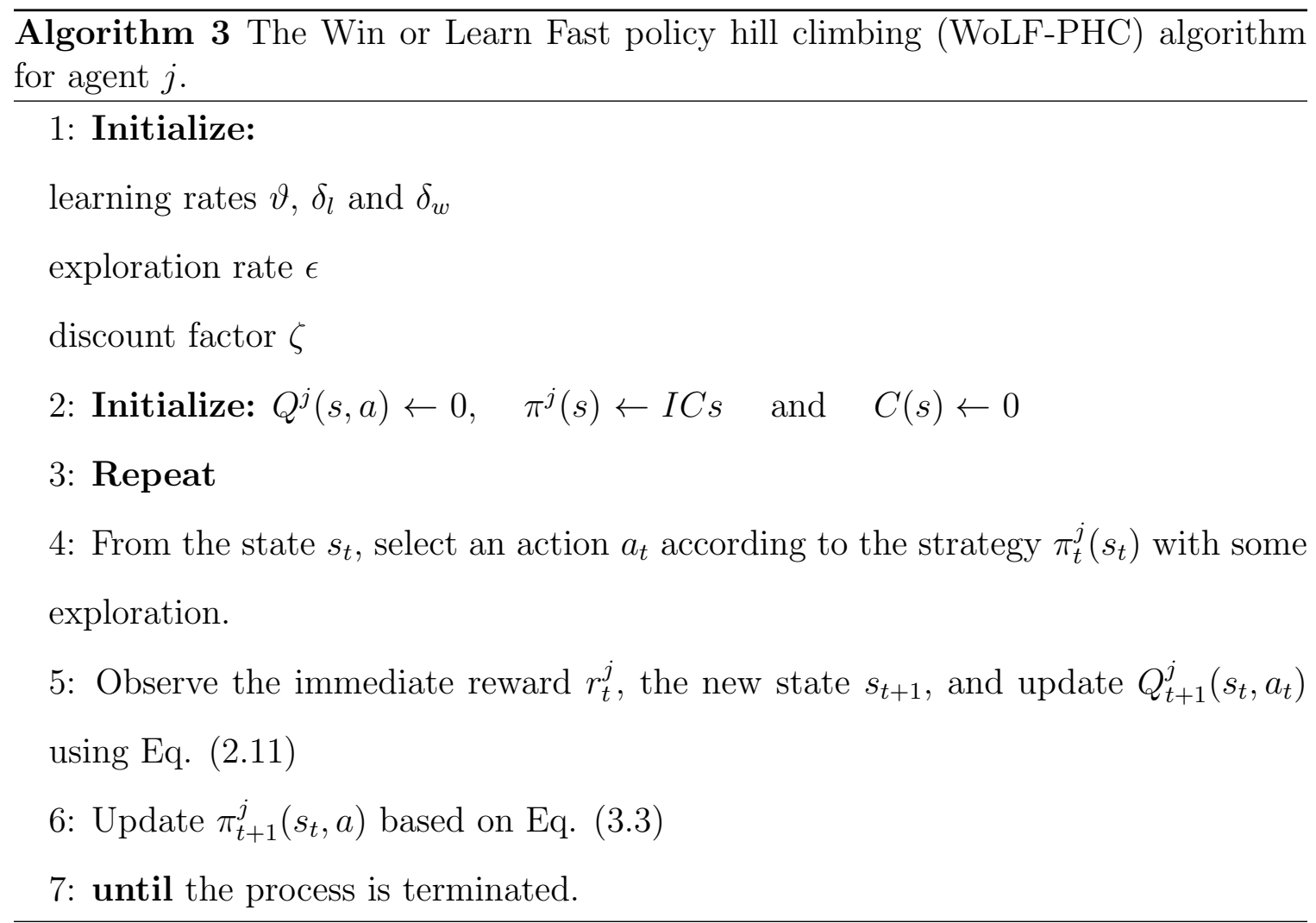


The WoLF-PHC algorithm updates the Q-table of the learning agent $j$ by Eq. (2.11), and updates the strategy of the agent $j$ by the following equation [9],

$$
\pi_{t+1}^{j}\left(s_{t}, a\right)=\pi_{t}^{j}\left(s_{t}, a\right)+\Delta_{s a},
$$

where,

$$
\begin{gathered}
\Delta_{s a}=\left\{\begin{array}{cc}
\delta & \text { if } a=\operatorname{argmax}_{a^{\prime}} Q_{t}^{j}\left(s_{t}, a^{\prime}\right), \\
\frac{-\delta}{\left|A_{j}\right|-1} & \text { otherwise, }
\end{array}\right. \\
\delta=\left\{\begin{array}{c}
\delta_{w} \quad \text { if } \sum_{a^{\prime}} \pi_{t}^{j}\left(s_{t}, a^{\prime}\right) Q_{t}^{j}\left(s_{t}, a^{\prime}\right)>\sum_{a^{\prime}} \bar{\pi}_{t}^{j}\left(s_{t}, a^{\prime}\right) Q_{t}^{j}\left(s_{t}, a^{\prime}\right), \\
\delta_{l} \quad \text { otherwise, } \\
\bar{\pi}_{t+1}^{j}\left(s_{t}, a^{\prime}\right)=\bar{\pi}_{t}^{j}\left(s_{t}, a^{\prime}\right)+\frac{1}{C\left(s_{t}\right)}\left[\pi_{t}^{j}\left(s_{t}, a^{\prime}\right)-\bar{\pi}_{t}^{j}\left(s_{t}, a^{\prime}\right)\right] \\
C\left(s_{t}\right)=C\left(s_{t}\right)+1,
\end{array} \quad \forall a^{\prime} \in A^{j},\right.
\end{gathered}
$$

where $\bar{\pi}\left(s_{t}, a\right)$ is the average policy and $C\left(s_{t}\right)$ is the number of visits to the state $s_{t}$. Algorithm 3 describes the complete procedure of the WoLF-PHC algorithm for the learning agent $j$.

\subsubsection{Summary and Discussion}

In this section, we reviewed three different decentralized MARL algorithms; the PGAAPP, the WPL, and the WoLF-PHC algorithms. The PGA-APP algorithm succeeds to converge to Nash equilibrium in some matrix games and fails to converge to Nash equilibrium in other matrix games as shown later in this chapter. The PGA-APP algorithm also fails to converge to Nash equilibrium in stochastic games considered in this thesis. The PGA-APP algorithm does not have a mathematical proof of convergence. The WPL algorithm performs better than the PGA-APP algorithm in matrix games, but it also fails to converge to Nash equilibrium in stochastic games considered in this 
Table 3.1: A comparison among the PGA-APP, the WPL, and the WoLF-PHC algorithms in terms of convergence to Nash equilibrium at some games.

\begin{tabular}{|c|c|c|c|c|c|}
\hline Algorithm Converge to NE & dilemma & shapley's & biased & grid 1 & grid 2 \\
\hline PGA-APP & Yes & Yes & No & No & No \\
\hline WPL & Yes & Yes & Close & No & No \\
\hline WoLF-PHC & Yes & No & Close & Yes & Yes \\
\hline
\end{tabular}

thesis. Moreover, the WPL algorithms does not have a formal mathematical proof of convergence; it has a numerical analysis of convergence instead. The WoLF-PHC algorithm, on the other hand, is a practical algorithm and shows a good convergence performance in a variety of matrix and stochastic games. However, it fails to converge to Nash equilibrium in some matrix games as shown later in this chapter. Likewise the PGA-APP and the WPL algorithms, the WoLF-PHC algorithm does not have a mathematical proof of convergence. Table 3.1 shows a comparison among the PGA-APP, the WPL, and the WoLF-PHC algorithms in terms of convergence to Nash equilibrium at some games. The notation "Yes" in the table indicates that the corresponding algorithm converges to Nash equilibrium at the corresponding game. The notation "No" in the table indicates that the corresponding algorithm does not converge to Nash equilibrium at the corresponding game. On the other hand, the notation "Close" in the table indicates that the corresponding algorithm converges to values that are close to Nash equilibrium at the corresponding game. 


\subsection{The Proposed Constant Learning Rate-based Exponential Moving Average Q-Learning (CLR-EMAQL) Algorithm}

The exponential moving average (EMA) approach is a model-free strategy estimation approach. It is one of the statistical approaches used to analyze time series data in finance and technical analysis. Typically, EMA gives the recent observations more weight than the older ones [13]. The EMA estimation approach is used in [21] by the hyper Q-learning algorithm to estimate the opponent's strategy. It is also used in [13] to estimate the opponent's strategy of the Infinitesimal Gradient Ascent (IGA) agent. The EMA estimator used to estimate the strategy of the agent's opponent can be described by the following equation $[13,21]$ :

$$
\pi_{t+1}^{-j}\left(s_{t}\right)=\left(1-\eta_{c}\right) \pi_{t}^{-j}\left(s_{t}\right)+\eta_{c} \mathbf{v}_{t}^{-j}\left(s_{t}\right)
$$

where $\pi^{-j}\left(s_{t}\right)$ is the opponent's strategy at the state $s_{t}, \eta_{c}$ is a small constant step size and $0<\eta_{c}<<1$, and $\mathbf{v}^{-j}\left(s_{t}\right)$ is a unit vector representation of the action $a^{-j}$ chosen by the opponent $(-j)$ at the state $s_{t}$. The unit vector $\mathbf{v}^{-j}\left(s_{t}\right)$ contains the same number of elements that $\pi^{-j}$ has. The elements in the unit vector $\mathbf{v}^{-j}\left(s_{t}\right)$ are all equal to zero except for the element corresponding to the action $a^{-j}$ which is equal to 1 . For example, if the opponent $(-j)$ has four possible actions at each state and the opponent chooses the second action at the state $s_{t}$, the unit vector $\mathbf{v}^{-j}\left(s_{t}\right)$ will be given in this case as follows, $\mathbf{v}^{-j}\left(s_{t}\right)=[0,1,0,0]$.

In this section, we propose the constant learning rate-based exponential moving average Q-learning (CLR-EMAQL) algorithm. The proposed CLR-EMAQL algorithm uses the exponential moving average (EMA) approach in parallel with the Q-learning 
algorithm as a basis to update the strategy of the learning agent itself. The CLREMAQL algorithm proposed in this section uses a constant learning rate $\eta_{c}$ (constant step size). The Q-table of a learning agent $j$ is updated by the Q-learning algorithm of Eq. (2.11). Despite the loss of theoretical guarantees, Q-learning agents often succeed to learn Nash equilibrium policies in a multi-agent environment [23]. This is because the Q-tables of the learning agents do not have to converge to optimal values in order for the agents to execute a Nash equilibrium policy, and the learning agents must adopt a Nash equilibrium if they are playing optimally [23]. It is important here to mention that the proposed algorithm forces the Q-table of the learning agent to converge to a Nash equilibrium policy. When the Q-table of the learning agent converges to a Nash equilibrium policy, the policy $\pi$ of the learning agent will also converge to a Nash equilibrium. The proposed CLR-EMAQL algorithm updates the agent $j$ 's policy by Eq. (3.5). Algorithm 4 lists the procedure of the CLR-EMAQL algorithm for a learning agent $j$ when using a constant learning rate $\eta_{c}$.

$$
\pi_{t+1}^{j}\left(s_{t}\right)=\left(1-\eta_{c}\right) \pi_{t}^{j}\left(s_{t}\right)+\eta_{c} \mathbf{v}_{t}^{j}\left(s_{t}\right)
$$

where $\eta_{c} \in(0,1)$ is a constant learning rate and $\mathbf{v}_{t}^{j}\left(s_{t}\right)$ is defined as follows,

$$
\mathbf{v}_{t}^{j}\left(s_{t}\right)=\left[\begin{array}{lllll}
v_{1}^{j} & v_{2}^{j} & . & . & v_{\mathfrak{M}}^{j}
\end{array}\right]^{T}= \begin{cases}\mathcal{V}_{1}^{j}\left(s_{t}\right) & \text { if } a_{t}=\underset{a^{\prime}}{\arg \max } Q_{t}^{j}\left(s_{t}, a^{\prime}\right), \\
\mathcal{V}_{2}^{j}\left(s_{t}\right) & \text { otherwise. }\end{cases}
$$

The elements $\left(v_{1}^{j}, v_{2}^{j}, \ldots, v_{\mathfrak{M}}^{j}\right) \in[0,1], v_{1}^{j}+v_{2}^{j}+\ldots+v_{\mathfrak{M}}^{j}=1$, and $\mathfrak{M}$ is the number of actions of the agent $j$. The vectors $\mathcal{V}_{1}^{j}\left(s_{t}\right)$ and $\mathcal{V}_{2}^{j}\left(s_{t}\right)$ consist of the same number of elements as $\pi^{j}$. The elements in the vector $\mathcal{V}_{1}^{j}\left(s_{t}\right)$ are all equal to zero except for the element corresponding to the action $a_{t}$ which is equal to 1 . On the other hand, $\mathcal{V}_{2}^{j}\left(s_{t}\right)=\frac{1}{\mathfrak{M}-1}\left[\overrightarrow{1}-\mathcal{V}_{1}^{j}\left(s_{t}\right)\right]$. When the action $a_{t}$ chosen by the agent $j$ at 
the state $s_{t}$ is equal to the greedy action obtained from the agent's Q-table at the state $s_{t}$, the term $\mathbf{v}_{t}^{j}\left(s_{t}\right)$ will equal to the vector $\mathcal{V}_{1}^{j}\left(s_{t}\right)$. On the other hand, if the learning agent selects an action that is different from the greedy action, the term $\mathbf{v}_{t}^{j}\left(s_{t}\right)$ will equal to the vector $\mathcal{V}_{2}^{j}\left(s_{t}\right)$. To illustrate the definition of the vectors $\mathcal{V}_{1}^{j}\left(s_{t}\right)$ and $\mathcal{V}_{2}^{j}\left(s_{t}\right)$ more, let us assume that, for example, agent $j$ has four possible actions at each state. Let us also assume that the action $a_{t}$ chosen by agent $j$ at the state $s_{t}$ is the third action. The vector $\mathbf{v}_{t}^{j}\left(s_{t}\right)$ will be defined in this case as $\mathbf{v}_{t}^{j}\left(s_{t}\right)=\mathcal{V}_{1}^{j}\left(s_{t}\right)=\left[\begin{array}{llll}v_{1}^{j} & v_{2}^{j} & v_{3}^{j} & v_{4}^{j}\end{array}\right]^{T}=[0,0,1,0]^{T}$ if the greedy action obtained from the agent's Q-table at the state $s_{t}$ is also the third action. On the other hand, the vector $\mathbf{v}_{t}^{j}\left(s_{t}\right)$ will be defined as $\mathbf{v}_{t}^{j}\left(s_{t}\right)=\mathcal{V}_{2}^{j}\left(s_{t}\right)=\left[\begin{array}{llll}v_{1}^{j} & v_{2}^{j} & v_{3}^{j} & v_{4}^{j}\end{array}\right]^{T}=[1 / 3,1 / 3,0,1 / 3]^{T}$ if the greedy action obtained from the agent's Q-table at the state $s_{t}$ is not the third action.

In the proposed CLR-EMAQL algorithm, the learning agent selects an action each time during learning based on his policy distribution $\pi(s)$. That is, the learning agent selects an action each time during learning and that action may not be the greedy action. The proposed CLR-EMAQL algorithm uses the greedy action as a criterion when it updates the policy of the learning agent. If the learning agent selects an action that is the same as the greedy action calculated from the Q-table of the Q-learning algorithm, then the proposed algorithm drags the agent's policy towards that action by giving the probability distribution corresponding to the selected action more weight than the other actions of the agent. We call this procedure by the operation of exploiting the greedy action by the CLR-EMAQL algorithm. If the selected action by the agent and the greedy action are different, the proposed algorithm then allows the learning agent to explore the other actions by decreasing the probability distribution of the selected action. We call this procedure by the operation of exploring actions by the CLR-EMAQL algorithm. The operation of 


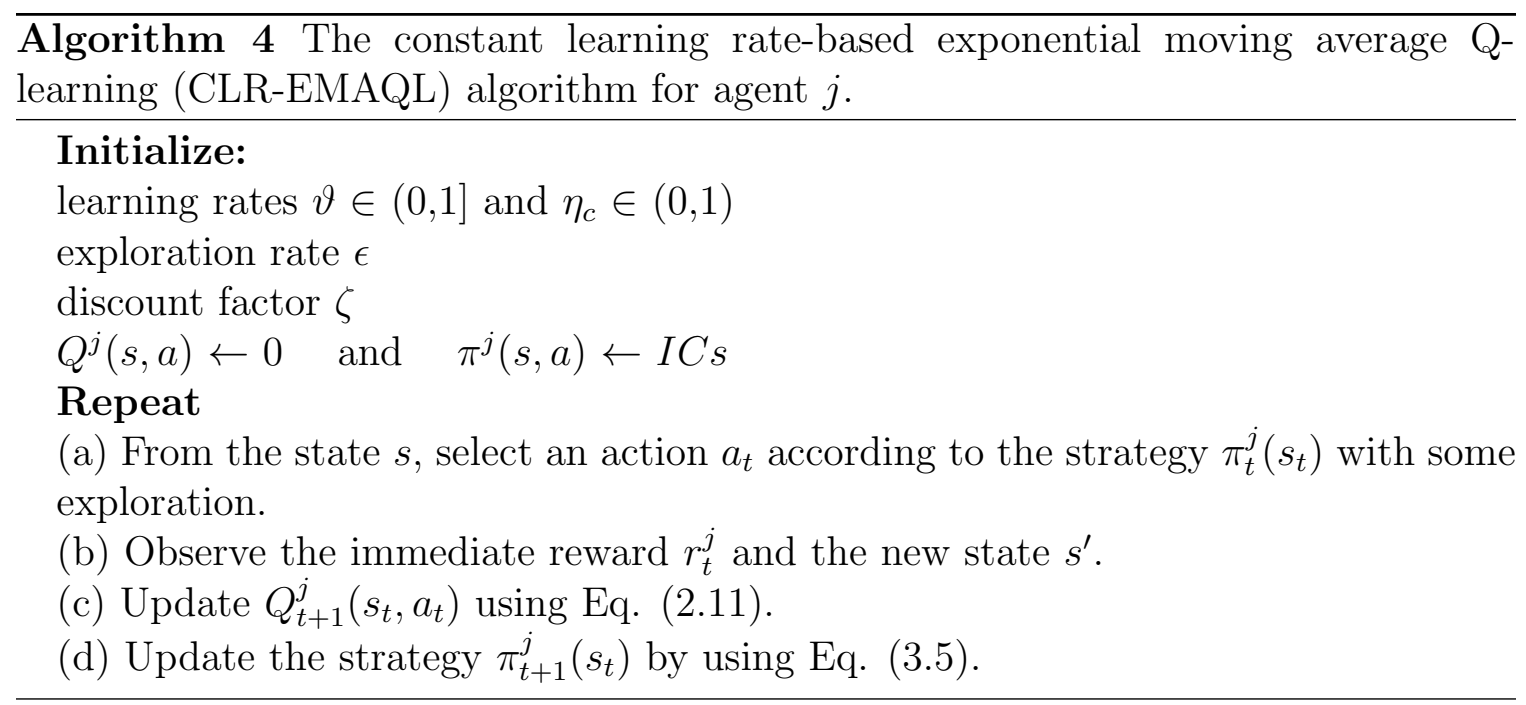

exploring the other actions continue until the learning agent selects an action that is the same as the greedy action calculated from the Q-table. At that point, the operation of exploiting the greedy action takes place and the proposed algorithm keeps updating the policy of the learning agent towards that greedy action by giving more weights to the probability distribution corresponding to that selected action.

\subsubsection{The Multi-agent Learning Dynamics of the Proposed CLR-EMAQL Algorithm}

To simplify the analysis, we consider two-player-two-action games. The policies of Player 1 and Player 2 updated by Eq. (3.5) can be written as follows,

$$
\begin{aligned}
& \pi_{t+1}^{1}\left(s_{t}\right)=\left(1-\eta_{c}\right) \pi_{t}^{1}\left(s_{t}\right)+\eta_{c} \mathbf{v}_{t}^{1}\left(s_{t}\right), \\
& \pi_{t+1}^{2}\left(s_{t}\right)=\left(1-\eta_{c}\right) \pi_{t}^{2}\left(s_{t}\right)+\eta_{c} \mathbf{v}_{t}^{2}\left(s_{t}\right),
\end{aligned}
$$


where $\mathbf{v}_{t}^{1}\left(s_{t}\right)$ and $\mathbf{v}_{t}^{2}\left(s_{t}\right)$ are defined based on Eq. (3.6) as follows,

$$
\begin{aligned}
& \mathbf{v}_{t}^{1}\left(s_{t}\right)= \begin{cases}\mathcal{V}_{1}^{1}\left(s_{t}\right) & \text { if } a_{t}=\underset{a^{\prime}}{\arg \max } Q_{t}^{1}\left(s_{t}, a^{\prime}\right), \\
\mathcal{V}_{2}^{1}\left(s_{t}\right) & \text { otherwise, }\end{cases} \\
& \mathbf{v}_{t}^{2}\left(s_{t}\right)= \begin{cases}\mathcal{V}_{1}^{2}\left(s_{t}\right) & \text { if } a_{t}=\underset{a^{\prime}}{\arg \max } Q_{t}^{2}\left(s_{t}, a^{\prime}\right), \\
\mathcal{V}_{2}^{2}\left(s_{t}\right) & \text { otherwise, }\end{cases}
\end{aligned}
$$

where $Q_{t}^{1}\left(s_{t}, a\right)$ and $Q_{t}^{2}\left(s_{t}, a\right)$ are the Q-tables for Player 1 and Player 2, respectively.

From Eq. (3.9) and Eq. (3.10), it is shown that $\mathbf{v}_{t}^{1}\left(s_{t}\right)$ is a function of $Q_{t}^{1}\left(s_{t}, a\right)$ and $\mathbf{v}_{t}^{2}\left(s_{t}\right)$ is a function of $Q_{t}^{2}\left(s_{t}, a\right)$. That is,

$$
\begin{aligned}
& \mathbf{v}_{t}^{1}\left(s_{t}\right)=f_{1}\left(Q_{t}^{1}\left(s_{t}, a\right)\right), \\
& \mathbf{v}_{t}^{2}\left(s_{t}\right)=f_{2}\left(Q_{t}^{2}\left(s_{t}, a\right)\right) .
\end{aligned}
$$

From Eq. (2.11), it is shown that the Q-tables of Player 1 and Player 2 are functions of the player's action and the opponent's action as well. This is because the reward $r_{t}^{j}$ of the player $j$ depends on the player $j$ 's action and the opponent's action. Thus,

$$
\begin{aligned}
& Q_{t}^{1}\left(s_{t}, a\right)=g_{1}\left(s_{t}, a^{1}, a^{2}\right), \\
& Q_{t}^{2}\left(s_{t}, a\right)=g_{2}\left(s_{t}, a^{1}, a^{2}\right),
\end{aligned}
$$

where $a^{1}$ and $a^{2}$ are the actions of Player 1 and Player 2, respectively. 
Hence, from Eq. (3.13) and Eq. (3.14), Eq. (3.11) and Eq. (3.12) can be rewritten as follows,

$$
\begin{aligned}
& \mathbf{v}_{t}^{1}\left(s_{t}\right)=f_{1}\left(Q_{t}^{1}\left(s_{t}, a\right)\right)=f_{1}\left(g_{1}\left(s_{t}, a^{1}, a^{2}\right)\right), \\
& \mathbf{v}_{t}^{2}\left(s_{t}\right)=f_{2}\left(Q_{t}^{2}\left(s_{t}, a\right)\right)=f_{2}\left(g_{2}\left(s_{t}, a^{1}, a^{2}\right)\right) .
\end{aligned}
$$

From Eq. (3.15) and Eq. (3.16), we can say that the policy equations of Player 1 and Player 2 given in Eq. (3.7) and Eq. (3.8) represent multi-agent learning equations. The convergence of these equations to a Nash equilibrium depends on the convergence of the Q-tables of Player 1 and Player 2. Although the Q-learning algorithm is a single-agent learning algorithm, it has been successfully used for multiagent learning $[26,27,39]$. Despite the loss of theoretical guarantees, Q-learning agents often succeed to learn Nash equilibrium policies in multi-agent environment [23]. This is because the Q-tables of the learning agents do not have to converge to optimal values in order for the agents to execute a Nash equilibrium policy. In addition, the learning agents must adopt a Nash equilibrium if they are playing optimally [23]. In

this work, the proposed algorithm forces the Q-table of the learning agent to converge to a Nash equilibrium policy. When the Q-table of the learning agent converges to a Nash equilibrium policy, the strategy (policy) of the learning agent will also converge to Nash equilibrium.

\subsubsection{The Mathematical Analysis of the Proposed CLR- EMAQL Algorithm}

To simplify the analysis, we consider two-player-two-action games. The probability of selecting the first action of Player 1 at time $t$ is referred to by $p_{1, t}$, whereas the 
probability of selecting the second action is referred to by $p_{2, t}$. Thus, the policy of Player 1 at state $s_{t}$ and time $t$ will be $\pi_{t}^{1}\left(s_{t}\right)=\left(p_{1, t}, p_{2, t}\right)$, where $p_{1, t}+p_{2, t}=1$. Similar to Player 1, the probability of selecting the first action of Player 2 at time $t$ is referred to by $q_{1, t}$, whereas the probability of selecting the second action at time $t$ is referred to by $q_{2, t}$. Thus, the policy of Player 2 at state $s_{t}$ and time $t$ will be $\pi_{t}^{2}\left(s_{t}\right)=\left(q_{1, t}, q_{2, t}\right)$, where $q_{1, t}+q_{2, t}=1$. To simplify notations, the term $\mathbf{v}_{t}^{j}\left(s_{t}\right)$ in Eq. (3.5) is defined as $\mathbf{v}_{t}^{1}\left(s_{t}\right)=\left[\begin{array}{ll}v_{1}^{1} & v_{2}^{1}\end{array}\right]^{T}$ for Player 1 and $\mathbf{v}_{t}^{2}\left(s_{t}\right)=\left[\begin{array}{ll}v_{1}^{2} & v_{2}^{2}\end{array}\right]^{T}$ for Player 2, where the superscripts refer to the corresponding player and the subscripts refer to the corresponding action. Hence, the policies of Player 1 and Player 2 updated by Eq. (3.5) can be rewritten as,

$$
\left[\begin{array}{l}
p_{1, t+1} \\
p_{2, t+1} \\
q_{1, t+1} \\
q_{2, t+1}
\end{array}\right]=\left[\begin{array}{cccc}
1-\eta_{c} & 0 & 0 & 0 \\
0 & 1-\eta_{c} & 0 & 0 \\
0 & 0 & 1-\eta_{c} & 0 \\
0 & 0 & 0 & 1-\eta_{c}
\end{array}\right]\left[\begin{array}{c}
p_{1, t} \\
p_{2, t} \\
q_{1, t} \\
q_{2, t}
\end{array}\right]+\left[\begin{array}{c}
\eta_{c} v_{1}^{1} \\
\eta_{c} v_{2}^{1} \\
\eta_{c} v_{1}^{2} \\
\eta_{c} v_{2}^{2}
\end{array}\right] .
$$

To analyze the above equation, we use the ordinary differential equation (ODE) approach. The behavior of the learning algorithm can be approximated by ODEs as the step size goes to zero [12]. Thus, when the learning rate (step size) $\eta_{c} \rightarrow 0$, the ordinary differential equation of Eq. (3.17) can be given as follows, 


$$
\left[\begin{array}{c}
\dot{p_{1}} \\
\dot{p_{2}} \\
\dot{q_{1}} \\
\dot{q_{2}}
\end{array}\right]=\left[\begin{array}{c}
v_{1}^{1}-p_{1} \\
v_{2}^{1}-p_{2} \\
v_{1}^{2}-q_{1} \\
v_{2}^{2}-q_{2}
\end{array}\right] .
$$

When the Q-table of each learning agent converges to a Nash equilibrium policy, the above ordinary differential equation can be viewed as a linear time-invariant equation. This linear time-invariant equation will be asymptotically stable as its eigenvalues are all real and less than zero, and they are $\left[\begin{array}{llll}-1-1 & -1-1\end{array}\right]^{T}$. If we let the right hand side of the above equation equal to zero, we then get the equilibrium solutions of the above equation as $p_{1}^{*}=v_{1}^{1}, p_{2}^{*}=v_{2}^{1}, q_{1}^{*}=v_{1}^{2}$, and $q_{2}^{*}=v_{2}^{2}$. For a two-player-two-action game, each parameter of the vector $\mathbf{v}_{t}^{1}\left(s_{t}\right)=\left[\begin{array}{ll}v_{1}^{1} & v_{2}^{1}\end{array}\right]^{T}$ for Player 1 will have a value of either zero or one, as stated in Eq. (3.5). Similar to Player 1, each parameter of the vector $\mathbf{v}_{t}^{2}\left(s_{t}\right)=\left[\begin{array}{ll}v_{1}^{2} & v_{2}^{2}\end{array}\right]^{T}$ for Player 2 will also have a value of either zero or one. Therefore, the possible equilibrium solutions of Eq. (3.18) can be specified as follows,

$$
\begin{aligned}
& \left(\pi^{1 *}, \pi^{2 *}\right)=((1,0),(1,0)), \\
& \left(\pi^{1 *}, \pi^{2 *}\right)=((1,0),(0,1)), \\
& \left(\pi^{1 *}, \pi^{2 *}\right)=((0,1),(1,0)), \\
& \left(\pi^{1 *}, \pi^{2 *}\right)=((0,1),(0,1)) .
\end{aligned}
$$

Without loss of generality, let us assume that the last equilibrium solution, $\left(\pi^{1 *}, \pi^{2 *}\right)=((0,1),(0,1))$, is the Nash equilibrium. Let us also assume that both players are adopting this Nash equilibrium. This means that selecting the second 
action by each player (Player 1 and Player 2) with a probability of one is the Nash equilibrium. This also means that the Q-table of each player updated by Eq. (2.11) converges to a Nash equilibrium policy so that the second action is the greedy action of each player's Q-table. Hence, none of the first three equilibrium solutions of Eq. (3.19) will be the Nash equilibrium solution as the proposed CLR-EMAQL algorithm of each player drags the player's policy away from these equilibrium solutions. Only the equilibrium solution $\left(\pi^{1 *}, \pi^{2 *}\right)=((0,1),(0,1))$ will be the Nash equilibrium of the game.

The equilibrium solution $\left(\pi^{1 *}, \pi^{2 *}\right)=((1,0),(1,0))$ : This equilibrium solution means that Player 1 selects its first action with a probability of one $\left(p_{1}=1, p_{2}=0\right)$, and Player 2 also selects its first action with a probability of one $\left(q_{1}=1, q_{2}=0\right)$. Since the greedy action of Player 1's Q-table is the second action, the term $\mathbf{v}_{t}^{1}\left(s_{t}\right)$ of Player 1 will be defined as stated in Eq. (3.5) as follows,

$$
\mathbf{v}_{t}^{1}\left(s_{t}\right)=\left[\begin{array}{ll}
v_{1}^{1} & v_{2}^{1}
\end{array}\right]^{T}=\left[\begin{array}{ll}
0 & 1
\end{array}\right]^{T}
$$

On the other hand, because the greedy action of Player 2's Q-table is the second action, the term $\mathbf{v}_{t}^{2}\left(s_{t}\right)$ of Player 2 will be defined as stated in Eq. (3.5) as follows,

$$
\mathbf{v}_{t}^{2}\left(s_{t}\right)=\left[\begin{array}{ll}
v_{1}^{2} & v_{2}^{2}
\end{array}\right]^{T}=\left[\begin{array}{ll}
0 & 1
\end{array}\right]^{T} .
$$

Hence, Eq. (3.18) can be defined as follows,

$$
\left[\begin{array}{l}
\dot{p_{1}} \\
\dot{p_{2}} \\
\dot{q_{1}} \\
\dot{q_{2}}
\end{array}\right]=\left[\begin{array}{c}
-p_{1} \\
1-p_{2} \\
-q_{1} \\
1-q_{2}
\end{array}\right] .
$$


The steady state values of Eq. (3.20) can be calculated by setting the right half side of the equation equal to zero. Thus, the steady state values of Eq. (3.20) are given as follows, $p_{1}^{*}=0, p_{2}^{*}=1, q_{1}^{*}=0$ and $q_{2}^{*}=1$. This means that the equilibrium solution $\left(\pi^{1 *}, \pi^{2 *}\right)=((1,0),(1,0))$ is not the Nash equilibrium. This is because the proposed CLR-EMAQL algorithm of each player drags the player's policy away from this equilibrium solution.

We can also do a similar analysis to the other equilibrium solutions given in Eq. (3.19). The analysis will show that only the equilibrium solution $\left(\pi^{1 *}, \pi^{2 *}\right)=((0,1),(0,1))$ will be the Nash equilibrium of the game. This is because the proposed CLR-EMAQL algorithm of each player will drag the player's policy towards this equilibrium solution.

In a two-player-two-action game, we have mathematically shown that the players learning with the proposed CLR-EMAQL algorithm successfully converge to Nash equilibrium if the game has a pure Nash equilibrium. However, in a two-player-two-action game with a mixed Nash equilibrium, the players learning with the proposed CLR-EMAQL algorithm will fail to adopt a mixed Nash equilibrium. The strategies of both players will keep oscillating around the mixed Nash equilibrium. This is also true in games with more than two players and with two actions or more. The strategies of the players learning with the proposed CLR-EMAQL algorithm will either converge to the Nash equilibrium if the game has a pure Nash strategy or oscillate around it if the game has a mixed Nash strategy.

To illustrate the performance of the proposed CLR-EMAQL algorithm, the algorithm is used to learn the games depicted in Fig. (3.1); the coordination game and the matching pennies game. The coordination game has a pure Nash strategy that executes the second action with a probability of 1 , whereas the matching pennies 
(a) Coordination Game

$$
R^{1,2}=\left[\begin{array}{cc}
1,1 & 0,0 \\
0,0 & 2,2
\end{array}\right]
$$

(b) Matching Pennies Game

$$
R^{1,2}=\left[\begin{array}{cc}
1,-1 & -1,1 \\
-1,1 & 1,-1
\end{array}\right]
$$

Figure 3.1: Two matrix games.

game has a mixed strategy that executes each action with a probability of 0.5. Fig. (3.2)(a) illustrates the probability distributions of action 2 of both players (Player 1 and Player 2) when using the proposed CLR-EMAQL algorithm to learn the coordination game. As shown in this figure, both players converge to their pure Nash equilibrium. This shows that the equilibrium points of the linear time-invariant system of Eq. (3.18) are reached, where $p_{1}^{*}=v_{1}^{1}=0, p_{2}^{*}=v_{2}^{1}=1, q_{1}^{*}=v_{1}^{2}=0$, and $q_{2}^{*}=v_{2}^{2}=1$. On the other hand, Fig. (3.2)(b) illustrates the probability distributions of action 1 and action 2 of Player 1 (policy of Player 1) when using the proposed CLR-EMAQL algorithm to learn the matching pennies game. This figure shows that the proposed CLR-EMAQL algorithm enforces the policy of Player 1 to oscillate around the Nash equilibrium strategy. As stated earlier in this section, in case of mixed Nash equilibrium, the policy of a CLR-EMAQL player will continue oscillating around the mixed Nash equilibrium. In this case, a decaying learning rate has to be used so that the oscillation around the Nash equilibrium vanishes and both players adopt their mixed Nash equilibrium.

\subsection{The Proposed Exponential Moving Average Q-Learning (EMAQL) Algorithm}

In this section, we introduce the proposed exponential moving average Q-learning (EMAQL) algorithm. The proposed EMAQL algorithm uses two different decaying 
(a) Coordination game by CLR-EMAQL algorithm

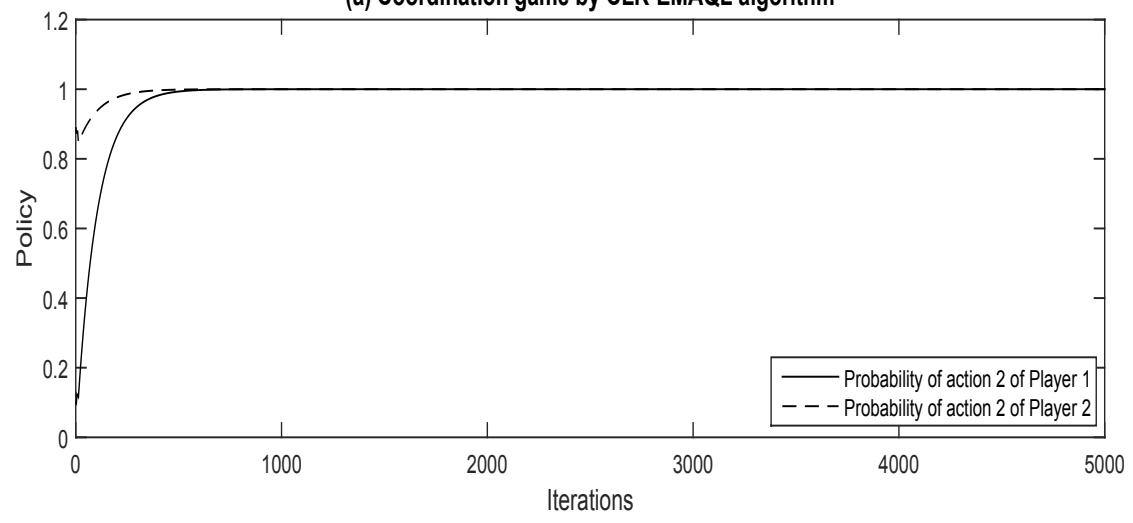

(b) Matching pennies game by CLR-EMAQL algorithm

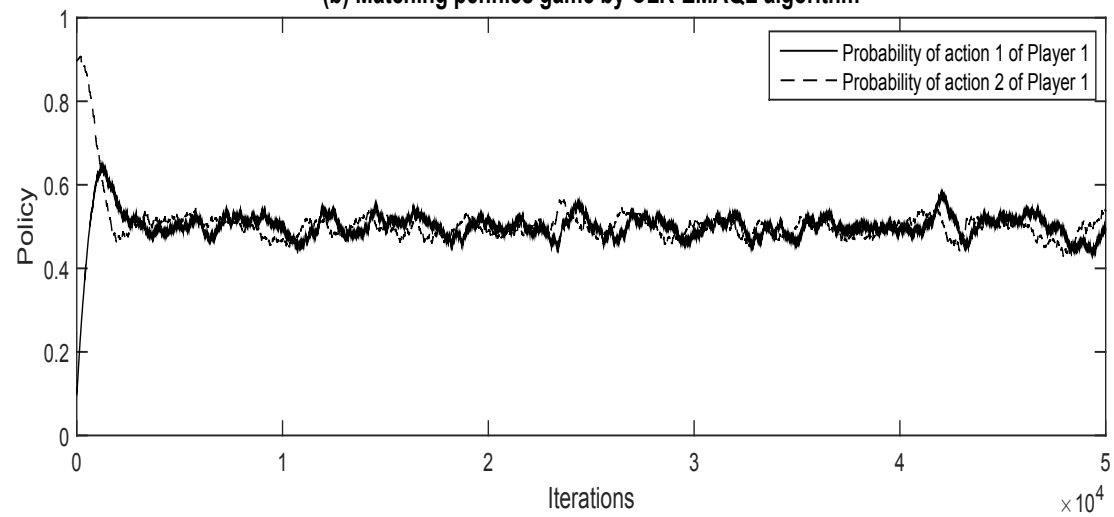

Figure 3.2: Probability of selecting actions: (a) Probability of choosing the second action for both players in the coordination game (b) Probability of choosing Player 1's actions in the the matching pennies game.

learning rates $\left(\eta_{w}\right.$ and $\left.\eta_{l}\right)$ when updating the agent's strategy instead of only one constant learning rate $\eta_{c}$ as used in the proposed CLR-EMAQL algorithm. The values of these variable learning rates are inversely proportional to the number of iterations (or episodes) and are set based on one of two different mechanisms; the Win-or-LearnFast (WoLF) mechanism or the Win-or-Learn-Slow (WoLS) mechanism. The Q-table of a learning agent $j$ is updated by the Q-learning algorithm of Eq. (2.11) and the agent $j$ 's policy is updated by Eq. (3.21) as follows,

$$
\pi_{t+1}^{j}\left(s_{t}\right)=\left(1-\eta_{t}\right) \pi_{t}^{j}\left(s_{t}\right)+\eta_{t} \mathbf{v}_{t}^{j}\left(s_{t}\right)
$$


where $\eta_{t}$ is a decaying learning rate and $\eta_{t} \in(0,1)$. The term $\mathbf{v}_{t}^{j}\left(s_{t}\right)$ and the learning rate $\eta_{t}$ are defined as follows,

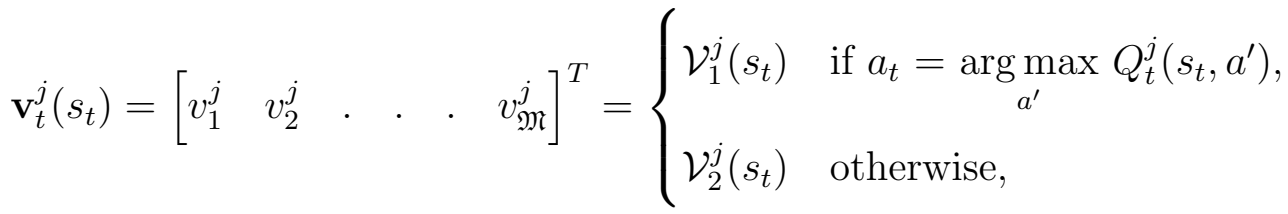

$$
\begin{aligned}
& \eta_{t}= \begin{cases}\eta_{w} & \text { if } a_{t}=\underset{a^{\prime}}{\arg \max } Q_{t}^{j}\left(s_{t}, a^{\prime}\right) \\
\eta_{l} & \text { otherwise }\end{cases}
\end{aligned}
$$

where,

$$
\eta_{l}= \begin{cases}\eta_{l}^{W o L F} & \text { if WoLF mechanism is used } \\ \eta_{l}^{W o L S} & \text { if WoLS mechanism is used }\end{cases}
$$

\subsubsection{WoLF and WoLS Mechanisms}

The WoLF principle was used with reinforcement learning algorithms in $[9,41,42]$. The WoLF mechanism used by the WoLF-PHC algorithm [9] uses two different learning rates, $\delta_{w}$ when the algorithm is winning and $\delta_{l}$ when it is losing. The WoLF-PHC algorithm uses the difference between the expected value of the average strategy and the expected value of the current strategy as a criterion to decide when the algorithm wins or loses. The learning rate $\delta_{l}$ is bigger than the learning rate $\delta_{w}$. As such, when the learning agent is losing, it learns faster than when it is winning. This makes the agent adapt quickly to the changes in the strategies when it is losing and to learn cautiously when it is winning [9]. In this work, the proposed EMAQL algorithm uses either the WoLF mechanism or the WoLS mechanism to update the policy of the learning agent. 
The WoLF mechanism used by the proposed EMAQL algorithm is similar to the WoLF mechanism used by the WoLF-PHC algorithm. It has two different learning rates $\left(\eta_{l}^{W o L F}\right.$ when losing and $\eta_{w}$ when winning), where $\eta_{l}^{W o L F}>\eta_{w}$. However, the criterion used by the proposed EMAQL algorithm to decide when the algorithm wins or loses is different from the criterion used by the WoLF-PHC algorithm. The proposed EMAQL algorithm uses the greedy action as a criterion to decide when the algorithm wins or loses. If the learning agent selects an action that is the same as the greedy action calculated from the Q-table of the learning agent, the algorithm wins and uses $\eta_{w}$. If the selected action is different from the greedy action, the algorithm loses and uses $\eta_{l}^{W o L F}$.

On the other hand, the WoLS mechanism used by the proposed EMAQL algorithm has two different learning rates $\left(\eta_{l}^{W o L S}\right.$ when losing and $\eta_{w}$ when winning), where $\eta_{l}^{W o L S}<\eta_{w}$. The essence of the WoLS mechanism is to learn fast when the algorithm wins and to learn cautiously when the algorithm loses. Hence, when the learning agent is losing, the policy of the learning agent is changed slowly. Learning cautiously when the algorithm is losing gives the algorithm the time to explore the other actions before deciding whether the current strategy (policy) is good for the learning agent or not. In other words, learning cautiously gives the learning agent the opportunity to see the response of the opponents to the learning agent's current strategy. If the opponents' response to the learning agent's current strategy benefits the learning agent, the algorithm will eventually transition from losing to winning when the greedy action obtained from the Q-table of the learning agent becomes similar to the action selected by the learning agent. On the other hand, if the opponents' response to the learning agent's current strategy does not benefit the learning agent, the algorithm will continue losing and, at the same time, exploring the other actions 
until a new strategy is adopted when the greedy action and the learning agent's selected action are similar to each other. At that time, the algorithm transitions from losing to winning.

Algorithm 5 lists the procedure of the proposed EMAQL algorithm for a learning agent $j$ when using a decaying learning rate $\eta_{t}$. As illustrated in the proposed CLR-EMAQL algorithm, when the Q-table of the learning agent converges to a Nash equilibrium policy, the policy of the learning agent will also converge to a Nash equilibrium. We will now illustrate how the proposed EMAQL algorithm works when the game has either a pure Nash equilibrium or mixed Nash equilibrium.

\subsubsection{Pure Nash Equilibrium}

For simplicity and without loss of generality, let us assume that we have a two-player game with some actions for each player. Let us also assume that each player is using the proposed EMAQL algorithm to learn its policy (self-play learning). When the learning starts and a joint action is selected by both players, then each player has two possible scenarios:

Scenario 1: The action selected by Player $j(j=1,2)$ is the same as the greedy action calculated from the Q-table of Player $j$. In this case, the proposed EMAQL algorithm of Player $j$ will update the player's policy so that it is dragged towards the player's greedy action by giving the probability distribution corresponding to the player's selected action more weight than the player's other actions. As stated in Algorithm 5, the player learning with the proposed EMAQL algorithm selects its action each time based on its policy distribution $\pi$ with some exploration. Player $j$ will continue updating its policy as stated in Scenario 1 as long as its selected action 
is the same as the greedy action calculated from Player $j$ 's Q-table. This is because the selected action is maximizing the player's Q-table corresponding to this action. That is, maximizing the player's payoff.

Scenario 2: The action selected by Player $j$ is different from the player's greedy action calculated from Player $j$ 's Q-table. In this case, the proposed EMAQL algorithm of Player $j$ will update the player's policy by decreasing the probability distribution of the player's selected action so that the player may explore its other actions. The amount of this reduction will depend on the updating policy mechanism used by the proposed EMAQL algorithm; the WoLF mechanism or the WoLS mechanism. This scenario will continue as long as the selected action and the greedy action are different. Once Player $j$ selects an action that is the greedy action, then the proposed EMAQL algorithm of Player $j$ will update the player's policy as stated in Scenario 1.

The operation of exploiting and exploring actions will continue by both players until a pure Nash equilibrium is adopted by both players. This pure Nash equilibrium will be reached through Scenario 1 when each player executes the selected action with a probability of one, and the selected action of each player is the same as the player's greedy action.

\subsubsection{Mixed Nash Equilibrium}

Likewise in the pure Nash equilibrium case, the proposed EMAQL algorithm of each player continues exploiting and exploring actions. As time goes on, the players' policies oscillate around the Nash equilibrium. This is because each player keeps changing its actions trying to maximize its payoff. Thus, both players' policies 


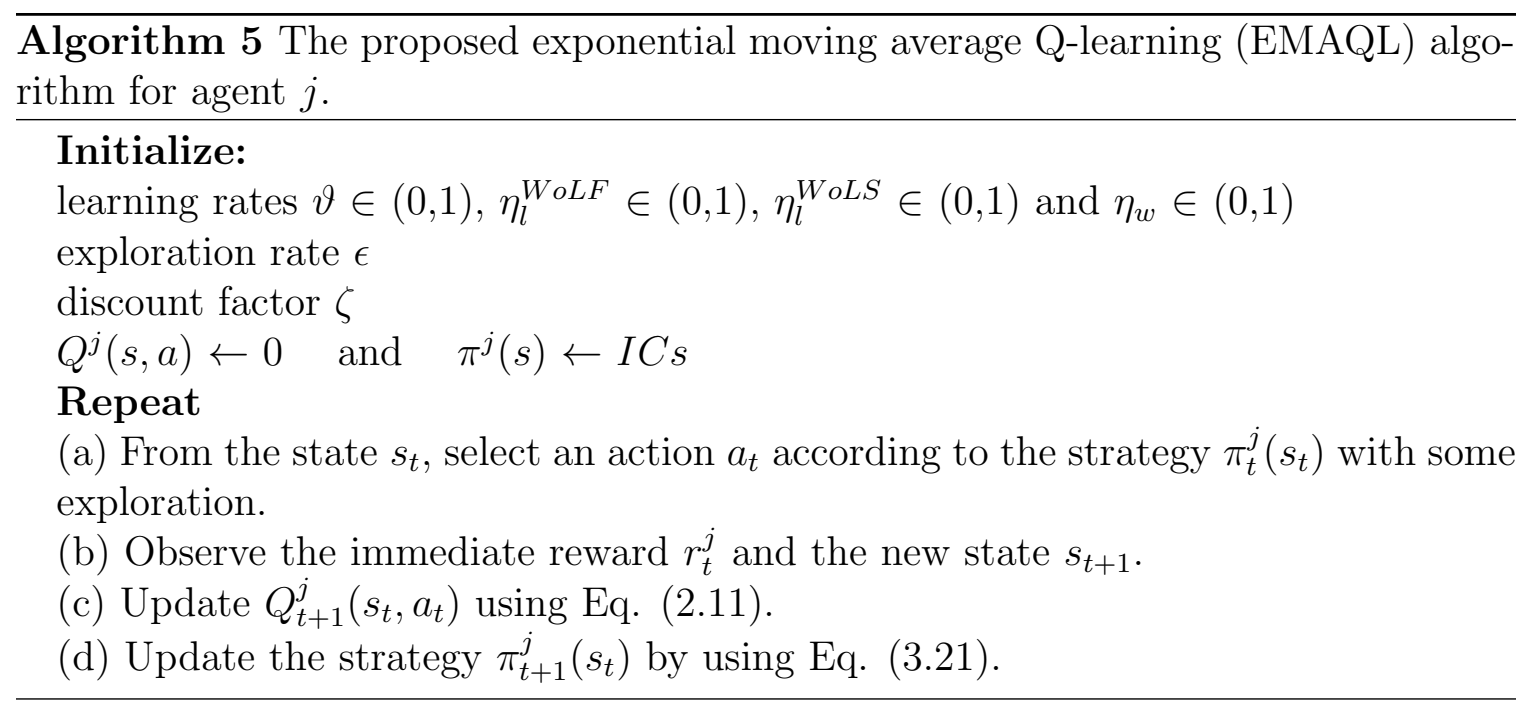

will keep oscillating around the mixed Nash equilibrium as both players keep changing their actions. In this case, the importance of decaying the learning rate (step size) $\eta_{t}$ of the proposed EMAQL algorithm arises. The more the learning rate $\eta_{t}$ decays, the less the oscillation of the players' policies around the Nash equilibrium becomes. Therefore, when the value of the decaying learning rate $\eta_{t}$ becomes zero, the oscillation of the players' policies around the Nash equilibrium becomes zero too. At this point, a mixed Nash equilibrium is adopted by both players.

\subsubsection{Nash Equilibrium $\pi^{*}(s)$}

The update rule of the policy iterate $\pi_{t+1}\left(s_{t}\right)$ in the proposed EMAQL algorithm is given as follows,

$$
\pi_{t+1}\left(s_{t}\right)=\left(1-\eta_{t}\right) \pi_{t}\left(s_{t}\right)+\eta_{t} \mathbf{v}_{t}\left(s_{t}\right)
$$

Let us for now assume that the strategy iterate $\pi_{t+1}\left(s_{t}\right)$ in Eq. (3.25) has a Nash equilibrium $\pi^{*}\left(s_{t}\right)$. Thus, when Eq. (3.25) converges to this Nash equilibrium $\pi^{*}\left(s_{t}\right)$, we get 


$$
\pi_{t+1}\left(s_{t}\right)=\pi_{t}\left(s_{t}\right)=\pi^{*}\left(s_{t}\right)
$$

From Eq. (3.25) and Eq. (3.26), we get

$$
\pi^{*}\left(s_{t}\right)=\left(1-\eta_{t}\right) \pi^{*}\left(s_{t}\right)+\eta_{t} \mathbf{v}_{t}\left(s_{t}\right)
$$

or,

$$
\pi^{*}\left(s_{t}\right)=\pi^{*}\left(s_{t}\right)+\eta_{t}\left[\mathbf{v}_{t}\left(s_{t}\right)-\pi^{*}\left(s_{t}\right)\right]
$$

Thus, for the proposed EMAQL algorithm to converge to Nash equilibrium, both sides of Eq. (3.27) must equal to each other. Therefore, at least one of the following conditions must be achieved:

Condition 1: $\pi^{*}\left(s_{t}\right)$ is equal to $\mathbf{v}_{t}\left(s_{t}\right)$. i.e.

$$
\pi_{t+1}\left(s_{t}\right) \underset{t \rightarrow \infty}{\rightarrow} \mathbf{v}_{t}\left(s_{t}\right)
$$

Condition 2: $\eta_{t}$ is equal to zero. i.e.

$$
\eta_{t} \underset{t \rightarrow \infty}{\rightarrow} 0
$$

When the Nash equilibrium $\pi^{*}\left(s_{t}\right)$ is a pure strategy, the proposed EMAQL algorithm will force the strategy iterate $\pi_{t+1}\left(s_{t}\right)$ of the learning agent to converge to its Nash equilibrium $\pi^{*}\left(s_{t}\right)$ so that Condition 1 is achieved, and when $t \rightarrow \infty$, Condition 2 is achieved too. However, in a game with a pure Nash equilibrium, Condition 2 does not have to be satisfied in order for an EMAQL agent to converge to its pure Nash equilibrium. On the other hand, when the Nash equilibrium $\pi^{*}\left(s_{t}\right)$ is a mixed strategy, the proposed EMAQL algorithm will force the strategy iterate $\pi_{t+1}\left(s_{t}\right)$ of 

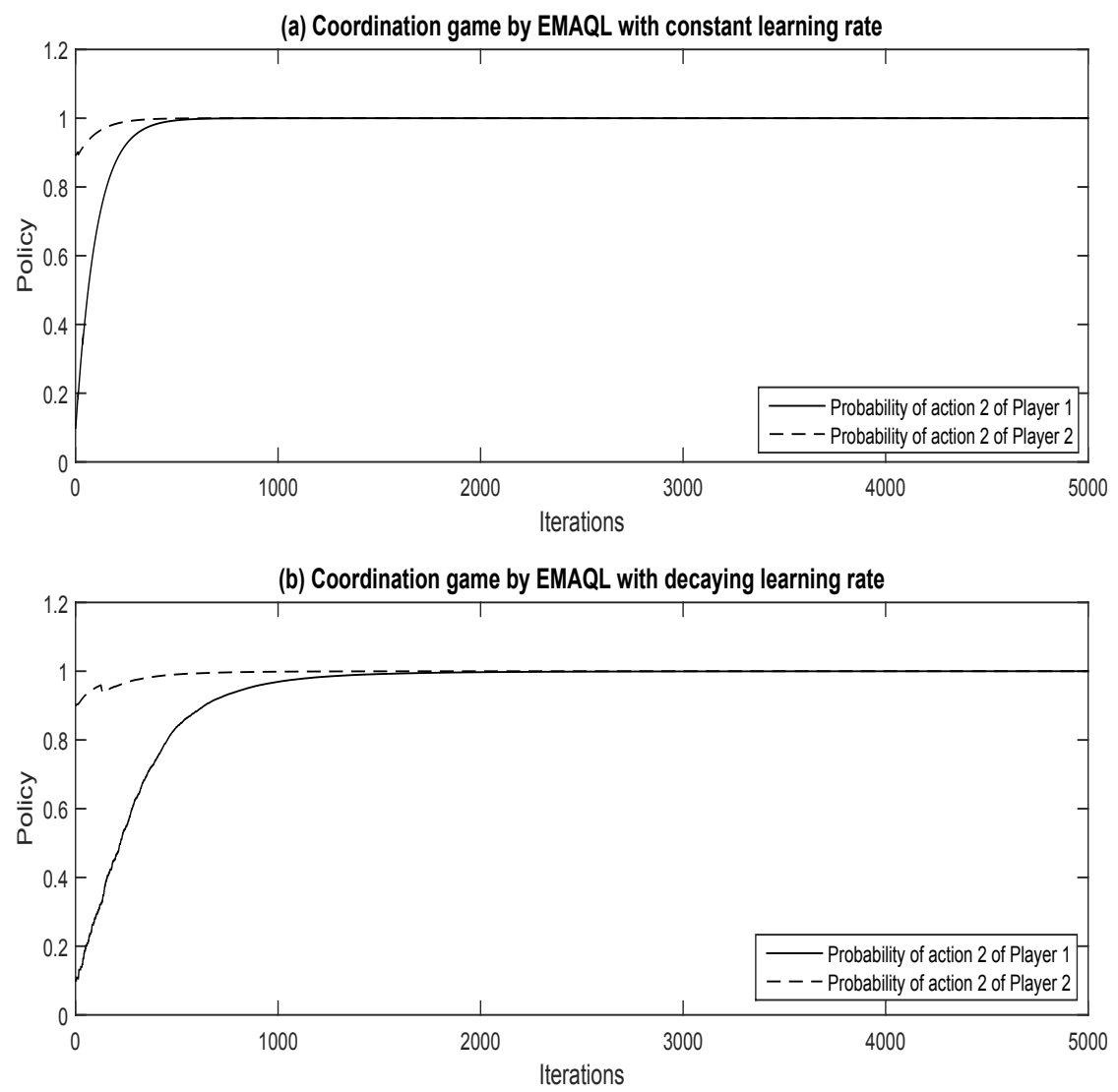

Figure 3.3: Probability of choosing the second action for both players in the coordination game: (a) when using a constant learning rate $\eta_{t}$. (b) when using a decaying learning rate $\eta_{t}$.

the learning agent to oscillate around its Nash equilibrium $\pi^{*}\left(s_{t}\right)$. When Condition 2 is achieved, the strategy iterate $\pi_{t+1}\left(s_{t}\right)$ converges to the Nash equilibrium $\pi^{*}\left(s_{t}\right)$.

To illustrate the effect of Condition 1 and Condition 2 on the convergence of the proposed EMAQL algorithm, the algorithm is used to learn the games depicted in Fig. (3.1); the coordination game and the matching pennies game. The coordination game has a pure Nash strategy that executes the second action with a probability of 1 , whereas the matching pennies game has a mixed strategy that executes each action with a probability of 0.5. Fig. (3.3)(a) illustrates the probability distributions of action 2 of both players (Player 1 and Player 2) when using the proposed EMAQL 


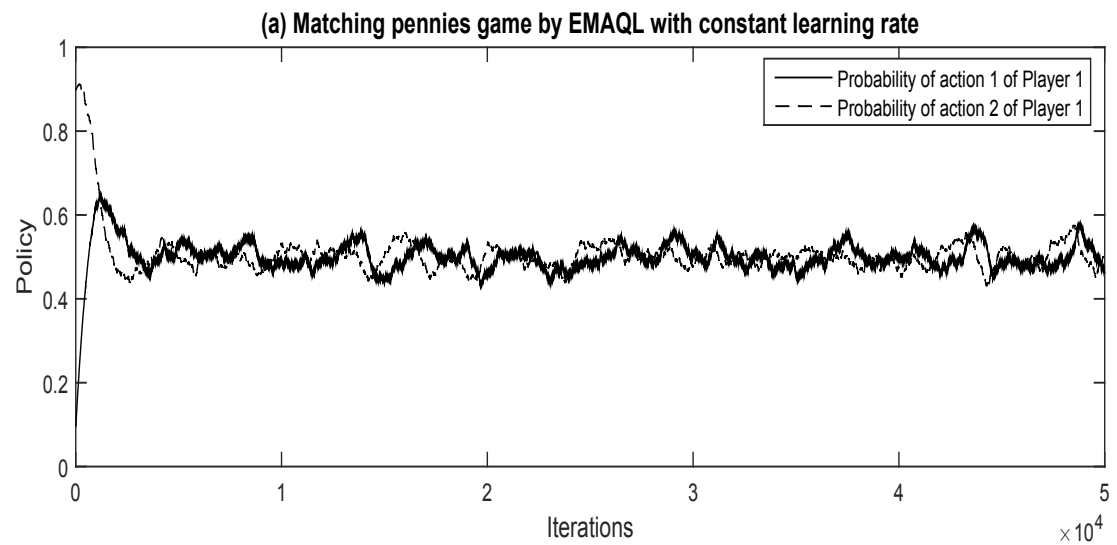

(b) Matching pennies game by EMAQL with decaying learning rate

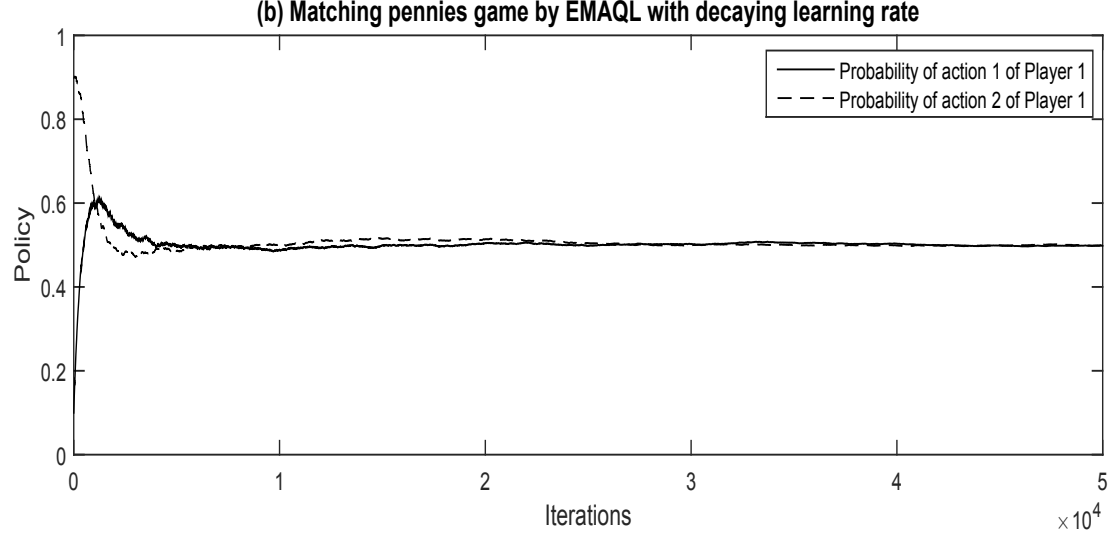

Figure 3.4: Probability of choosing Player 1's actions in the the matching pennies game: (a) when using a constant learning rate $\eta_{t}$. (b) when using a decaying learning rate $\eta_{t}$.

algorithm to learn the coordination game (a constant learning rate $\eta$ is used in this case). As shown in this figure, both players converge to their pure Nash equilibrium. This shows that Condition 1 is satisfied as each player' strategy converges to $\mathbf{v}_{t}\left(s_{t}\right)$, where $\mathbf{v}_{t}\left(s_{t}\right)=\left[\begin{array}{ll}0 & 1\end{array}\right]^{T}$ in this case. On the other hand, Fig. (3.3)(b) illustrates the probability distributions of action 2 of both players (Player 1 and Player 2) when using the proposed EMAQL algorithm to learn the coordination game (a decaying learning rate $\eta_{t}$ is used in this case). This figure shows that both players converge to their Nash equilibrium when Condition 2 is satisfied. Fig. (3.3) shows that in a game with a pure Nash equilibrium, an EMAQL player will converge to its Nash equilibrium when Condition 1 is satisfied even if Condition 2 is not satisfied. This 
figure also shows that the pure Nash equilibrium will be reached through Scenario 1 when each player executes the selected action with a probability of one, and the selected action of each player is the same as the player's greedy action. Fig. (3.4)(a) illustrates the probability distributions of action 1 and action 2 of Player 1 (policy of Player 1) in the matching pennies game when learning with the proposed EMAQL algorithm (a constant learning rate $\eta$ is used in this case). This figure shows that the proposed EMAQL algorithm forces the policy of Player 1 to oscillate around the Nash equilibrium strategy. As stated earlier, in case of mixed Nash equilibrium, the policy of the learning player will continue oscillating around the mixed Nash equilibrium when a constant learning rate $\eta$ is used. In this case a decaying learning rate has to be used so that the oscillation around the Nash equilibrium vanishes and both players adopt their mixed Nash equilibrium. Fig. (3.4)(b) shows that using the proposed EMAQL algorithm (with a decaying learning rate $\eta_{t}$ ) affects the learning performance and makes the policy of Player 1 converge to the mixed Nash equilibrium. Fig. (3.4)(b) shows that in a game with a mixed Nash equilibrium, an EMAQL player will converge to its Nash equilibrium when condition 2 is satisfied.

\subsubsection{The Mathematical Analysis of the Proposed EMAQL Algorithm}

To simplify analysis, we consider two-player-two-action games. However, what we are going to present here is also valid for any other multi-agent learning game. The probabilities of selecting actions at time $t$ are referred to by $p_{1, t}$ and $p_{2, t}$ for the first and the second actions of Player 1, respectively. Similar to Player 1, the probabilities of selecting actions are referred to by $q_{1, t}$ and $q_{2, t}$ for the first and the second actions of Player 2, respectively. Thus, the policy of Player 1 at state $s_{t}$ and time $t$ will be 
$\pi_{t}^{1}\left(s_{t}\right)=\left(p_{1, t}, p_{2, t}\right)$, where $p_{1, t}+p_{2, t}=1$. On the other hand, the policy of Player 2 at state $s_{t}$ and time $t$ will be $\pi_{t}^{2}\left(s_{t}\right)=\left(q_{1, t}, q_{2, t}\right)$, where $q_{1, t}+q_{2, t}=1$. To simplify notations, the term $\mathbf{v}_{t}^{j}\left(s_{t}\right)$ in Eq. (3.21) is defined as $\mathbf{v}_{t}^{1}\left(s_{t}\right)=\left[\begin{array}{ll}v_{1}^{1} & v_{2}^{1}\end{array}\right]^{T}$ for Player 1 and $\mathbf{v}_{t}^{2}\left(s_{t}\right)=\left[\begin{array}{ll}v_{1}^{2} & v_{2}^{2}\end{array}\right]^{T}$ for Player 2, where the superscripts refer to the corresponding player and the subscripts refer to the corresponding action. Without loss of generality, let us rewrite the equation of updating the policy iterate $\pi_{t+1}\left(s_{t}\right)$ given by Eq. (3.21) as follows,

$$
\pi_{t+1}^{j}\left(s_{t}\right)=\left(1-\eta_{c} \eta_{t}\right) \pi_{t}^{j}\left(s_{t}\right)+\eta_{c} \eta_{t} \mathbf{v}_{t}^{j}\left(s_{t}\right)
$$

where $\eta_{c}$ is a small constant step size and $\eta_{c} \eta_{t} \in(0,1)$.

Thus, the policies of Player 1 and Player 2 updated by Eq. (3.30) can be rewritten as follows,

$$
\left[\begin{array}{l}
p_{1, t+1} \\
p_{2, t+1} \\
q_{1, t+1} \\
q_{2, t+1}
\end{array}\right]=\left[\begin{array}{cccc}
1-\eta_{c} \eta_{t} & 0 & 0 & 0 \\
0 & 1-\eta_{c} \eta_{t} & 0 & 0 \\
0 & 0 & 1-\eta_{c} \eta_{t} & 0 \\
0 & 0 & 0 & 1-\eta_{c} \eta_{t}
\end{array}\right]\left[\begin{array}{c}
p_{1, t} \\
p_{2, t} \\
q_{1, t} \\
q_{2, t}
\end{array}\right]+\left[\begin{array}{c}
\eta_{c} \eta_{t} v_{1}^{1} \\
\eta_{c} \eta_{t} v_{2}^{1} \\
\eta_{c} \eta_{t} v_{1}^{2} \\
\eta_{c} \eta_{t} v_{2}^{2}
\end{array}\right]
$$

To analyze the above equation, we use the ordinary differential equation (ODE) approach. The behavior of the learning algorithm can be approximated by ODEs as the step size goes to zero [12]. Thus, when the step size $\eta_{c} \rightarrow 0$, the ordinary differential equation of Eq. (3.31) can be given as follows, 


$$
\left[\begin{array}{c}
\dot{p_{1}} \\
\dot{p_{2}} \\
\dot{q_{1}} \\
\dot{q_{2}}
\end{array}\right]=\left[\begin{array}{cccc}
-\eta_{t} & 0 & 0 & 0 \\
0 & -\eta_{t} & 0 & 0 \\
0 & 0 & -\eta_{t} & 0 \\
0 & 0 & 0 & -\eta_{t}
\end{array}\right]\left[\begin{array}{c}
p_{1} \\
p_{2} \\
q_{1} \\
q_{2}
\end{array}\right]+\left[\begin{array}{l}
\eta_{t} v_{1}^{1} \\
\eta_{t} v_{2}^{1} \\
\eta_{t} v_{1}^{2} \\
\eta_{t} v_{2}^{2}
\end{array}\right],
$$

or,

$$
\left[\begin{array}{c}
\dot{p_{1}} \\
\dot{p_{2}} \\
\dot{q_{1}} \\
\dot{q_{2}}
\end{array}\right]=\left[\begin{array}{c}
-\eta_{t} p_{1}+\eta_{t} v_{1}^{1} \\
-\eta_{t} p_{2}+\eta_{t} v_{2}^{1} \\
-\eta_{t} q_{1}+\eta_{t} v_{1}^{2} \\
-\eta_{t} q_{2}+\eta_{t} v_{2}^{2}
\end{array}\right]
$$

To find the equilibrium solutions of the the above ordinary differential equation, let the right hand side of Eq. (3.33) equal to zero. Because the parameters of the vectors $\mathbf{v}_{t}^{1}\left(s_{t}\right)$ and $\mathbf{v}_{t}^{2}\left(s_{t}\right)$ are either 0 or 1 , the possible equilibrium solutions of the above ordinary differential equation can be specified as follows, 


$$
\begin{aligned}
& \left(\pi^{1 *}, \pi^{2 *}\right)=((1,0),(1,0)), \\
& \left(\pi^{1 *}, \pi^{2 *}\right)=((1,0),(0,1)), \\
& \left(\pi^{1 *}, \pi^{2 *}\right)=((0,1),(1,0)), \\
& \left(\pi^{1 *}, \pi^{2 *}\right)=((0,1),(0,1)), \\
& \left(\pi^{1 *}, \pi^{2 *}\right)=\left(\left(p_{1}^{*}, p_{2}^{*}\right),\left(q_{1}^{*}, q_{2}^{*}\right)\right) .
\end{aligned}
$$

Hence, the ordinary differential equation of Eq. (3.32) (or Eq. (3.33)) will have either a pure Nash equilibrium when $\left[v_{1}^{1}-p_{1}, v_{2}^{1}-p_{2}, v_{1}^{2}-q_{1}, v_{2}^{2}-q_{2}\right]^{T}=0$ (Condition 1 in the previous subsection), or a mixed Nash equilibrium when $\eta_{t} \rightarrow 0$ (Condition 2 in the previous subsection).

Thus, the possible equilibrium solutions of the game can be specified as given in Eq. (3.34). Without loss of generality, let us assume that the game has a pure Nash equilibrium and the equilibrium solution $\left(\pi^{1 *}, \pi^{2 *}\right)=((0,1),(0,1))$ of Eq. (3.34) is the Nash equilibrium. Let us also assume that both players adopt this Nash equilibrium. That is, each player selects its second action with a probability of one. This also means that the greedy action of the Q-table of each player is the second action. Therefore, none of the first three equilibrium solutions of Eq. (3.34) will be the Nash equilibrium of the game. This is because of the same reasons we illustrate in the proposed CLR-EMAQL algorithm. The last equilibrium solution of Eq. (3.34), $\left(\pi^{1 *}, \pi^{2 *}\right)=\left(\left(p_{1}^{*}, p_{2}^{*}\right),\left(q_{1}^{*}, q_{2}^{*}\right)\right)$, will not be the Nash equilibrium of the game either. This is because this equilibrium solution means that Player 1 selects its actions with a probability of $\left(p_{1}=p_{1}^{*}, p_{2}=p_{2}^{*}\right)$, and Player 2 selects its actions with a probability of $\left(q_{1}=q_{1}^{*}, q_{2}=q_{2}^{*}\right)$. Since the greedy action of Player 1's Q-table is the second action, the term $\mathbf{v}_{t}^{1}\left(s_{t}\right)$ of Player 1 will be defined as stated in Eq. (3.21) as follows 
whatever the selected action by Player 1 is,

$$
\mathbf{v}_{t}^{1}\left(s_{t}\right)=\left[\begin{array}{ll}
v_{1}^{1} & v_{2}^{1}
\end{array}\right]^{T}=\left[\begin{array}{ll}
0 & 1
\end{array}\right]^{T}
$$

On the other hand, because the greedy action of Player 2's Q-table is the second action, the term $\mathbf{v}_{t}^{2}\left(s_{t}\right)$ of Player 2 will be defined as stated in Eq. (3.21) as follows whatever the selected action by Player 2 is,

$$
\mathbf{v}_{t}^{2}\left(s_{t}\right)=\left[\begin{array}{ll}
v_{1}^{2} & v_{2}^{2}
\end{array}\right]^{T}=\left[\begin{array}{ll}
0 & 1
\end{array}\right]^{T}
$$

Hence, Eq. (3.33) can be defined as follows,

$$
\left[\begin{array}{c}
\dot{p_{1}} \\
\dot{p_{2}} \\
\dot{q_{1}} \\
\dot{q_{2}}
\end{array}\right]=\eta_{t}\left[\begin{array}{c}
-p_{1} \\
1-p_{2} \\
-q_{1} \\
1-q_{2}
\end{array}\right] .
$$

The steady state values of Eq. (3.35) are $p_{1}^{*}=0, p_{2}^{*}=1, q_{1}^{*}=0$ and $q_{2}^{*}=1$. This means that the equilibrium solution $\left(\pi^{1 *}, \pi^{2 *}\right)=\left(\left(p_{1}^{*}, p_{2}^{*}\right),\left(q_{1}^{*}, q_{2}^{*}\right)\right)$ is not the Nash equilibrium of the game. This is because the proposed EMAQL algorithm of each player drags the player's policy away from this equilibrium solution. Hence, only the equilibrium solution $\left(\pi^{1 *}, \pi^{2 *}\right)=((0,1),(0,1))$ will be the Nash equilibrium of the game. This is because the proposed EMAQL algorithm of each player drags the player's policy towards this equilibrium solution.

Let us now assume that the two-player-two-action game has a mixed Nash equilibrium, and the equilibrium solution $\left(\pi^{1 *}, \pi^{2 *}\right)=\left(\left(p_{1}^{*}, p_{2}^{*}\right),\left(q_{1}^{*}, q_{2}^{*}\right)\right)$ of Eq. 
is the mixed Nash equilibrium of the game. Let us also assume that both players adopt this Nash equilibrium. That is, Player 1 selects its actions with a probability of $\left(p_{1}=p_{1}^{*}, p_{2}=p_{2}^{*}\right)$, and Player 2 selects its actions with a probability of $\left(q_{1}=q_{1}^{*}, q_{2}=q_{2}^{*}\right)$. The greedy action of the Q-table of each player will not be a specific action all the time. This is because, in mixed Nash equilibrium games, no player will benefit from using the same action with a probability of one all the time as its opponent (the other player) will take advantage of that and maximize its payoff. This will make both players keep changing their actions. Thus, none of the first four equilibrium solutions of Eq. (3.34) will be the Nash equilibrium of the game as the proposed EMAQL algorithm of each player will drag the player's policy away from adopting a pure strategy. Therefore, the strategy of each player will keep oscillating around the game's Nash equilibrium until both players adopt their mixed Nash equilibrium when $\eta_{t} \rightarrow 0$. Hence, the equilibrium solution $\left(\pi^{1 *}, \pi^{2 *}\right)=\left(\left(p_{1}^{*}, p_{2}^{*}\right),\left(q_{1}^{*}, q_{2}^{*}\right)\right)$ of Eq. (3.34) is the only Nash equilibrium of the game. It is important to mention here that, in the case of games with mixed Nash equilibrium, our mathematical analysis shows that the proposed EMAQL algorithm converges to an equilibrium. Although our mathematical analysis does not explicitly show that the proposed EMAQL algorithm converges to a Nash equilibrium in games with mixed Nash equilibrium, our simulation results, as will be presented later, indicate that the proposed EMAQL algorithm does converge to Nash equilibrium.

\subsubsection{The Stability of the Equilibrium Solutions of the Pro- posed EMAQL Algorithm}

In this subsection, we will study the stability of the equilibrium solutions of the proposed EMAQL algorithm when the game has a pure Nash equilibrium and when 
it has a mixed Nash equilibrium.

\section{When the Game Has a Pure Nash Equilibrium:}

In pure Nash equilibrium games, when the Q-table of each learning agent converges to a Nash equilibrium policy, the ordinary differential equations of Eq. (3.33) can be viewed as linear time-varying equations.

\section{Theorem 1:}

The first-order linear time-varying system [7] characterized by the following equation,

$$
\begin{gathered}
\lambda_{0}(t) \dot{x}(t)+\lambda_{1}(t) x(t)=\mathcal{M}(t), \\
x\left(t_{0}\right)=x_{0},
\end{gathered}
$$

has a transition matrix $\phi\left(t, t_{0}\right)$ described as follows:

$$
\phi\left(t, t_{0}\right)=\exp \left[-\int_{t_{0}}^{t} \frac{\lambda_{1}(\Gamma)}{\lambda_{0}(\Gamma)} \mathrm{d} \Gamma\right]
$$

where $t_{0}, x_{0}$ and $\mathcal{M}(t)$ are the initial time, the initial state and the system's input, respectively.

Each equation in Eq. (3.33) can be rewritten as follows,

$$
\dot{x}(t)+\eta_{t} x(t)=\eta_{t} v_{t}
$$

or, 


$$
\dot{x}(t)+\eta_{t} x(t)=\mathcal{M}(t) .
$$

From Eq. (3.36) and Eq. (3.38), we have,

$$
\frac{\lambda_{1}(t)}{\lambda_{0}(t)}=\eta_{t}
$$

Without loss of generality, let $t_{0}=0$ and $\eta_{t}=\frac{1}{c_{1}+c_{2} t}$, where $c_{1}$ and $c_{2}$ are constants. Thus,

$$
\begin{aligned}
\phi(t, 0) & =\exp \left[-\int_{0}^{t} \frac{\lambda_{1}(\Gamma)}{\lambda_{0}(\Gamma)} \mathrm{d} \Gamma\right] \\
& =\exp \left[-\int_{0}^{t} \eta_{\Gamma} \mathrm{d} \Gamma\right] \\
& =\exp \left[-\int_{0}^{t} \frac{1}{c_{1}+c_{2} \Gamma} \mathrm{d} \Gamma\right], \\
& =\exp \left[-\frac{1}{c_{2}} \ln \left|c_{1}+c_{2} \Gamma\right|_{0}^{t}\right], \\
& =\exp \left[-\frac{1}{c_{2}}\left(\ln \left|c_{1}+c_{2} t\right|-\ln \left|c_{1}\right|\right)\right], \\
& =\exp \left[-\frac{1}{c_{2}}\left(\ln \left|c_{1}+c_{2} t\right|+\ln \left|\frac{1}{c_{1}}\right|\right)\right] \\
& =\exp \left[-\frac{1}{c_{2}} \ln \left|\frac{c_{1}+c_{2} t}{c_{1}}\right|\right], \\
& =\exp \left[\ln \left|\frac{c_{1}+c_{2} t}{c_{1}}\right|^{-\frac{1}{c_{2}}}\right] \\
& =\exp \left[\ln \left|\frac{c_{1}}{c_{1}+c_{2} t}\right|^{\frac{1}{c_{2}}}\right] .
\end{aligned}
$$

Thus,

$$
\phi(t, 0)=\left(\frac{c_{1}}{c_{1}+c_{2} t}\right)^{\frac{1}{c_{2}}} .
$$

Therefore, from Eq. (3.39), the transition matrix of the diagonal matrix of the linear time-varying system of Eq. (3.32) is given as follows, 


$$
\phi(t, 0)=\left[\begin{array}{cccc}
\phi_{1}(t, 0) & 0 & 0 & 0 \\
0 & \phi_{1}(t, 0) & 0 & 0 \\
0 & 0 & \phi_{2}(t, 0) & 0 \\
0 & 0 & 0 & \phi_{2}(t, 0)
\end{array}\right],
$$

where $\phi_{1}(t, 0)=\left(\frac{a_{1}}{a_{1}+a_{2} t}\right)^{\frac{1}{a_{2}}}$ and $\phi_{2}(t, 0)=\left(\frac{b_{1}}{b_{1}+b_{2} t}\right)^{\frac{1}{b_{2}}}$.

\section{Theorem 2}

The equilibrium solution of the linear time-varying system $[6,7]$ given by the following equation,

$$
\dot{x}(t)=A(t) x(t)+B(t) \mathcal{M}(t)
$$

is (globally) uniformly asymptotically stable if and only if:

$$
\begin{aligned}
& \sup _{t_{0} \geq 0} \sup _{t \geq t_{0}}\left\|\phi\left(t, t_{0}\right)\right\|_{i}=\mathfrak{M}_{0}<\infty, \\
& \left\|\phi\left(t+t_{0}, t_{0}\right)\right\|_{i} \rightarrow 0 \text { as } t \rightarrow \infty,
\end{aligned}
$$

where the notation $\|.\|_{i}$ refers to some weighted maximum norm.

Hence, the pure Nash equilibrium of the linear time-varying system of Eq. (3.33) is (globally) uniformly asymptotically stable as $\sup _{t \geq 0}\|\phi(t, 0)\|_{i}<\infty$ and 
$\lim _{t \rightarrow \infty}\|\phi(t, 0)\|_{i} \rightarrow 0$

\section{When the Game Has a Mixed Nash Equilibrium:}

In mixed Nash equilibrium games, we view the term $\eta_{t} v$ as an input, $\mathcal{M}(t)$, to the differential equations of Eq. (3.33). Because the learning rate $\eta_{t}$ decays as time goes on, the input, $\mathcal{M}(t)$, to the differential equations of Eq. (3.33) becomes very small and continuous as well. At that point, the differential equations of Eq. (3.33) become linear time-varying differential equations. Hence, the stability analysis performed in case of pure Nash equilibrium games can be applied to these differential equations too.

\subsection{Simulation and Results}

We have evaluated the proposed EMAQL, the WoLF-PHC [9], the GIGA-WoLF [8], the WPL [1], and the PGA-APP [22] algorithms on a variety of matrix and stochastic games. Given that both the WoLF-PHC and the GIGA-WoLF algorithms give nearly identical results over the same games [8], we choose to only show the proposed EMAQL, the PGA-APP, the WPL and the WoLF-PHC algorithms. The results of applying the WoLF-PHC, the WPL, the PGA-APP and the proposed EMAQL algorithms to different matrix and stochastic games are presented in this section. A comparison among the four algorithms in terms of the convergence to Nash equilibrium is provided. The learning rates of each algorithm are carefully chosen based on trial and error basis for a number of combinations of these learning rates so that each algorithm achieves the best performance. 


\subsubsection{Matrix Games}

The proposed EMAQL, the PGA-APP, the WPL and the WoLF-PHC algorithms are applied to the matrix games depicted in Fig. (3.5). Fig. (3.6) shows the probabilities of selecting the second actions by both players in the dilemma game. The proposed EMAQL, the PGA-APP, the WPL, and the WoLF-PHC algorithms are shown. In this game, the parameters of the proposed EMAQL algorithm are set as follows, $\eta_{w}=\frac{1}{100+\iota / 25}, \eta_{l}^{W o L F}=2 \eta_{w}, \eta_{l}^{W o L S}=0.001 \eta_{w}, \zeta=0.25$, and $\vartheta=\frac{0.1}{1+0.0001 \iota}$ with an exploration rate $\epsilon=0.05$, where $\iota$ is the current number of episodes. The parameters of the WoLF-PHC algorithm are selected as those of the proposed EMAQL algorithm with $\delta_{w}=\frac{1}{100+\iota}$ and $\delta_{l}=2 \delta_{w}$. In the PGA-APP algorithm, the parameter $\zeta$ is set as $\zeta=0$, and the parameter $\gamma^{P}$ is set as $\gamma^{P}=0.5$. In the WPL algorithm, the parameters are set as follows, $\eta^{W}=0.05, \zeta=0$, and $\vartheta=0.01$. The dilemma game has a pure Nash equilibrium strategy that executes the second action of each player with a probability of one. As can be seen in Fig. (3.6), when learning with all different algorithms, both players adopt their Nash equilibrium strategy as the probabilities of selecting the second actions by both players converge to one after some time. The probabilities of selecting actions by both players in Fig. (3.6)(a) go through the scenarios we stated in Section 3.4.2 until a Nash equilibrium strategy is finally adopted by both players. Fig. (3.6)(a) confirms our claim in Section 3.4.2 that the pure Nash equilibrium is reached through Scenario 1 when the selected action of each player has a probability distribution of one, and at the same time this selected action is the same as the greedy action calculated from the Q-table of that player.

Fig. (3.7) shows the probability of choosing Player 1's actions in the shapley's game when learning with the proposed EMAQL, the PGA-APP, the WPL, and the 


$\begin{gathered}\text { Dilemma Game } \\ R^{1,2}=\left[\begin{array}{cc}10,10 & 1,12 \\ 12,1 & 2,2\end{array}\right] \quad R^{1,2}=\left[\begin{array}{ccc}0,0 & 1,0 & 0,1 \\ 0,1 & 0,0 & 1,0 \\ 1,0 & 0,1 & 0,0\end{array}\right]\end{gathered} \quad R^{1,2}=\left[\begin{array}{cc}1,1.85 & 1.85,1 \\ 1.15,1 & 1,1.15\end{array}\right]$

Figure 3.5: Matrix games.

WoLF-PHC algorithms. In this game, the parameters of the proposed EMAQL algorithm are set as follows, $\eta_{w}=\frac{1}{100+\iota}, \eta_{l}^{W o L F}=2 \eta_{w}, \eta_{l}^{W o L S}=0.001 \eta_{w}, \zeta=0.95$, and $\vartheta=\frac{2}{10+0.0001 \iota}$ with an exploration rate $\epsilon=0.05$. The learning rates of the WoLF-PHC algorithm are set as follows, $\delta_{w}=\frac{1}{100+\iota / 10}$ and $\delta_{l}=2 \delta w$. The parameters of the PGA-APP algorithm are set as follows, $\eta^{P}=\frac{1}{100+\iota / 50}$ and $\gamma^{P}=3$. On the other hand, the parameters of the WPL algorithm are set as follows, $\eta^{W}=\frac{1}{100+\iota / 500}$, $\zeta=0$, and $\vartheta=0.95$. The shapley's game has one mixed Nash equilibrium strategy that executes each action of each player with a probability of $\frac{1}{3}$. As can be seen in Fig. (3.7)(a), when learning with the proposed EMAQL algorithm, both players adopt their Nash equilibrium strategy as the probabilities of selecting each action of Player 1 converge to $\frac{1}{3}$ after some time. Fig. (3.7)(a) shows that the probabilities of selecting actions by Player 1 converge to their mixed Nash equilibrium when Condition 2 we stated in Section 3.4.4 is satisfied. Fig. (3.7)(b) and Fig. (3.7)(c) show that the PGA-APP and the WPL algorithms succeed to learn the Nash equilibrium of the shapley's game. Fig. $(3.7)(d)$, on the other hand, shows that the WoLF-PHC algorithm fails to learn the Nash equilibrium of the shapley's game.

Fig. (3.8) shows the probability of choosing the first action for both players in the biased game while learning with the proposed EMAQL, the PGA-APP, the WPL, and the WoLF-PHC algorithms. The biased game has a mixed Nash equilibrium strategy with probabilities not uniform across actions, $(0.15,0.85)$ and $(0.85,0.15)$. 
In this game, the parameters of the proposed EMAQL algorithm are set as follows:

$\eta_{w}=\frac{1}{10+\iota / 5}, \eta_{l}^{W o L F}=2 \eta_{w}, \eta_{l}^{W o L S}=0.001 \eta_{w}, \zeta=0.95$, and $\vartheta=\frac{1}{10+0.0001 \iota}$ with an exploration rate $\epsilon=0.05$. The WoLF-PHC algorithm's learning rates are set as $\delta_{w}=\frac{1}{10+\iota}$ and $\delta_{l}=2 \delta w$. In the PGA-APP algorithm, the values of $\zeta$ and $\gamma^{P}$ are set as follows: $\zeta=0$ and $\gamma^{P}=3$. In the WPL algorithm, the parameters are set as follows, $\zeta=0, \vartheta=0.01$ and $\eta^{W}=0.05$. Fig. (3.8)(a) shows that the proposed EMAQL algorithm succeeds to converge to a Nash equilibrium in the biased game. The players' strategies in this figure go through the scenarios stated in Sections 3.4.2 and 3.4.3 until a Nash equilibrium is adopted. Fig. (3.8)(b) shows that the PGAAPP algorithm fails to learn the Nash equilibrium of the biased game. Fig. (3.8)(c) and Fig. (3.8)(d), on the other hand, show that the WPL and the WoLF-PHC algorithms converge to values that are close to the Nash equilibrium of the biased game. In conclusion, simulation results presented in Fig. (3.6) to Fig. (3.8) show that the proposed EMAQL algorithm outperforms the state-of-the-art (PGA-APP, WPL, and WoLF-PHC) algorithms in terms of the convergence to Nash equilibrium in the matrix games depicted in Fig. (3.5).

\subsubsection{Stochastic Games}

The proposed EMAQL, the WoLF-PHC, the PGA-APP and the WPL algorithms are used to learn the grid games depicted in Fig. (3.9); grid game 1 and grid game 2. Each game of these grid games has more than one Nash equilibrium; grid game 1 has ten different Nash equilibria, whereas grid game 2 has two different Nash equilibria [16]. We run grid game 1 until the game converges to the Nash equilibrium shown in Fig. (3.10)(a). We also run grid game 2 until the game converges to the Nash equilibrium shown in Fig. (3.10)(b). In both games, we only show the probabilities of the players' actions when they first move from their initial state. This is to investigate whether or not the players' probabilities of the selected actions will 
(a) Dilemma game by EMAQL algorithm

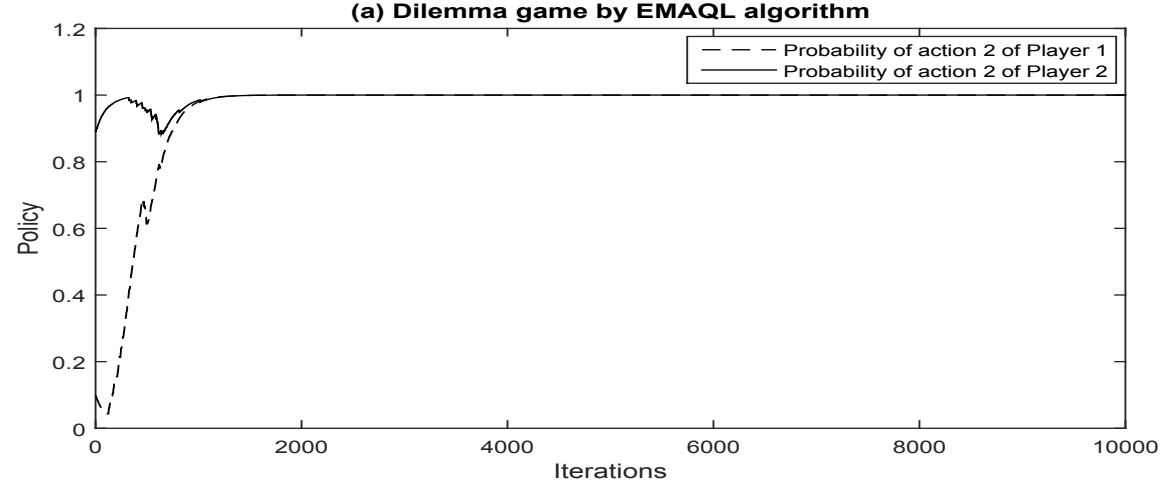

(b) Dilemma game by PGA-APP algorithm

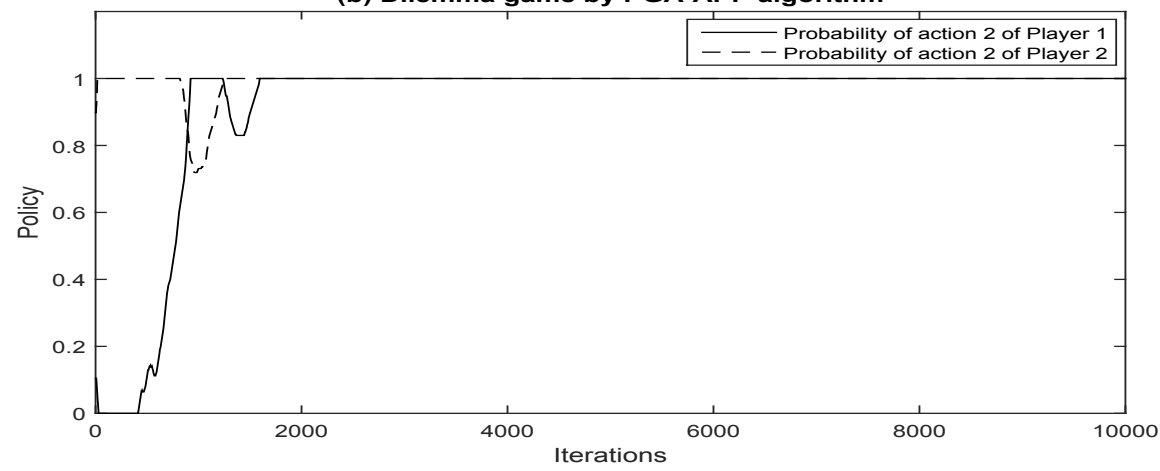

(c) Dilemma game by WPL algorithm

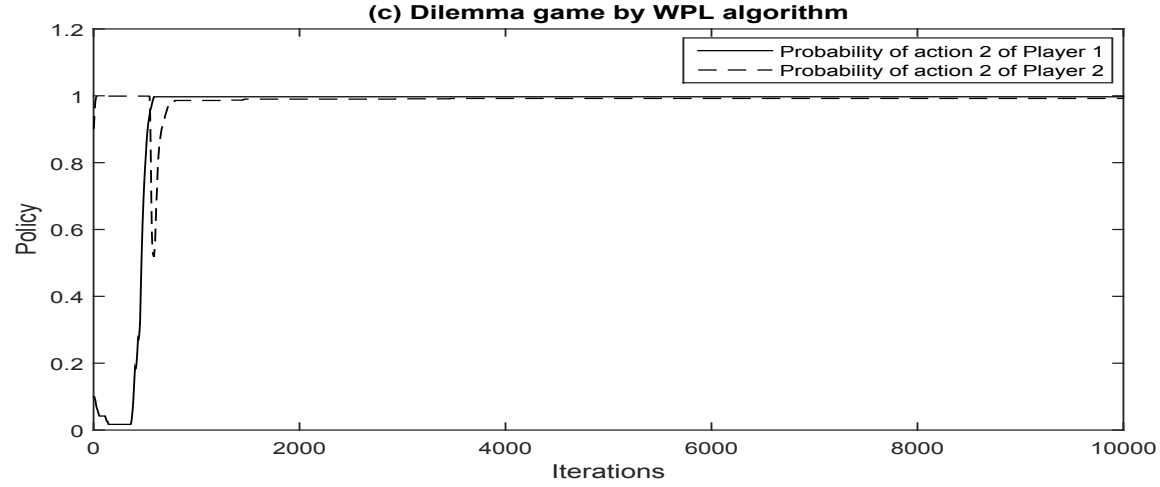

(d) Dilemma game by WoLF-PHC algorithm

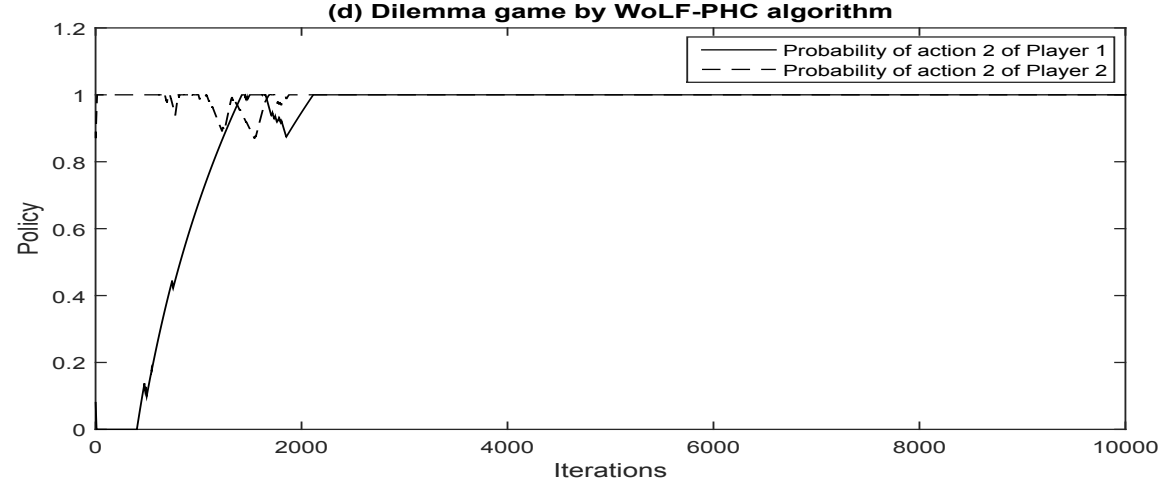

Figure 3.6: Probability of choosing the second action for both players in the dilemma game. The proposed EMAQL, the PGA-APP, the WPL, and the WoLF-PHC algorithms are shown. 
(a) Shapley's game by EMAQL algorithm

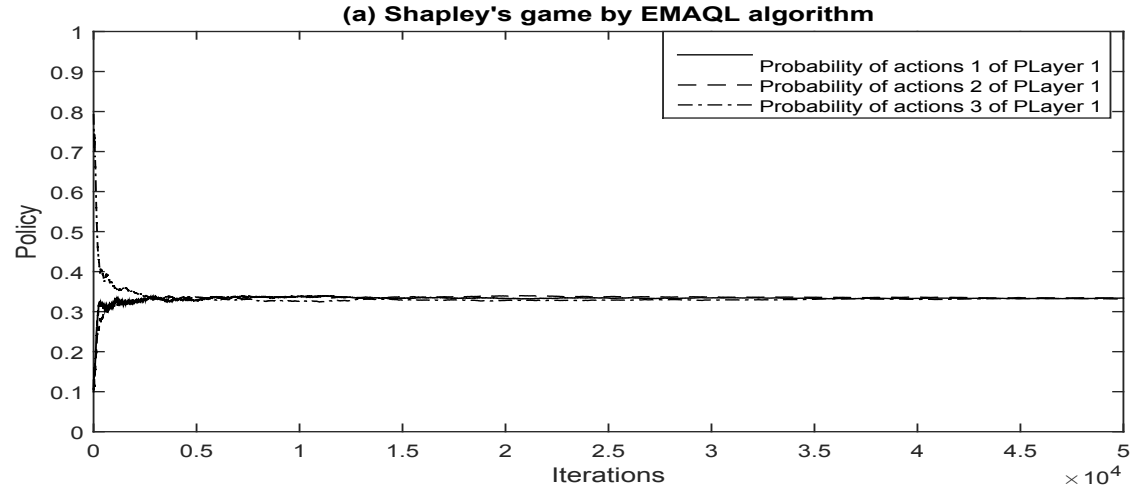

(b) Shapley's game by PGA-APP algorithm

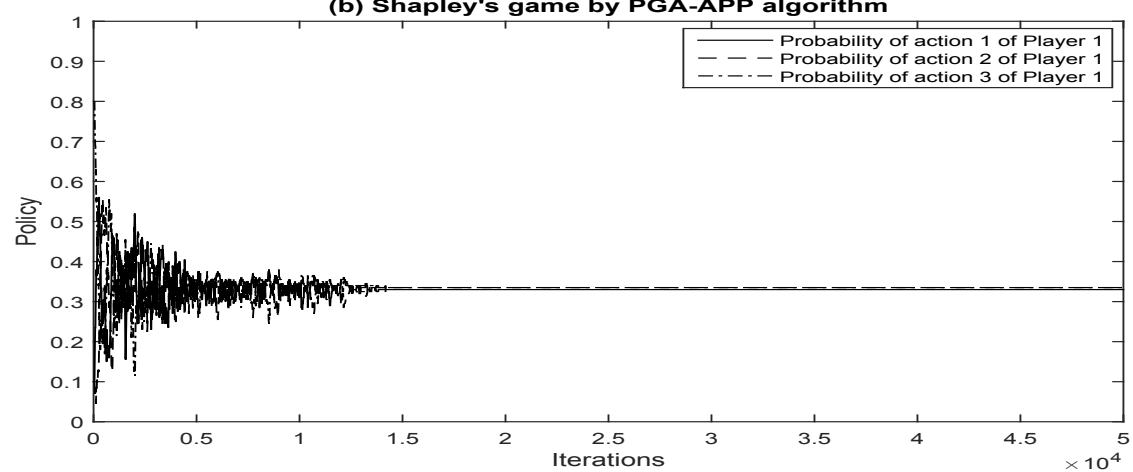

(c) Shapley's game by WPL algorithm

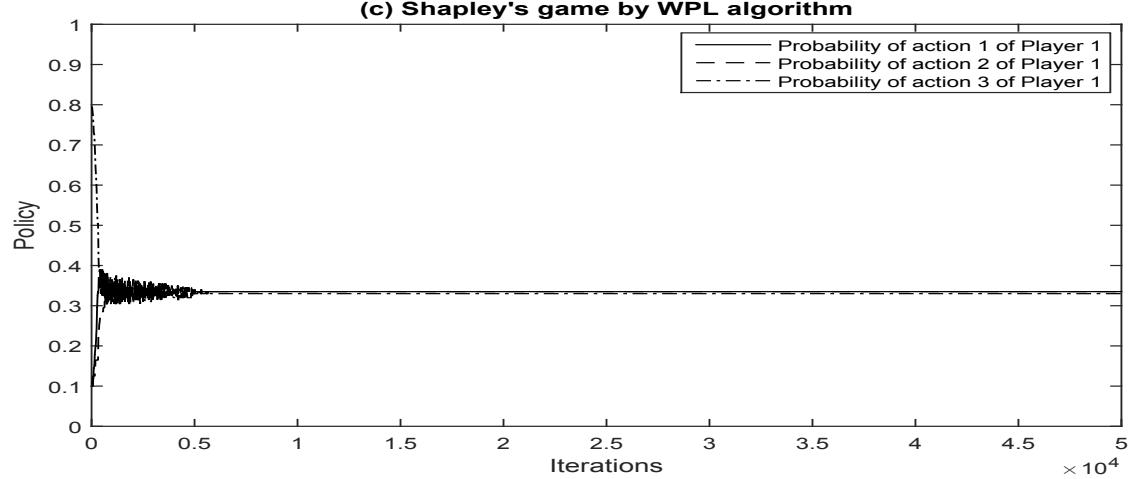

(d) Shapley's game by WoLF-PHC algorithm

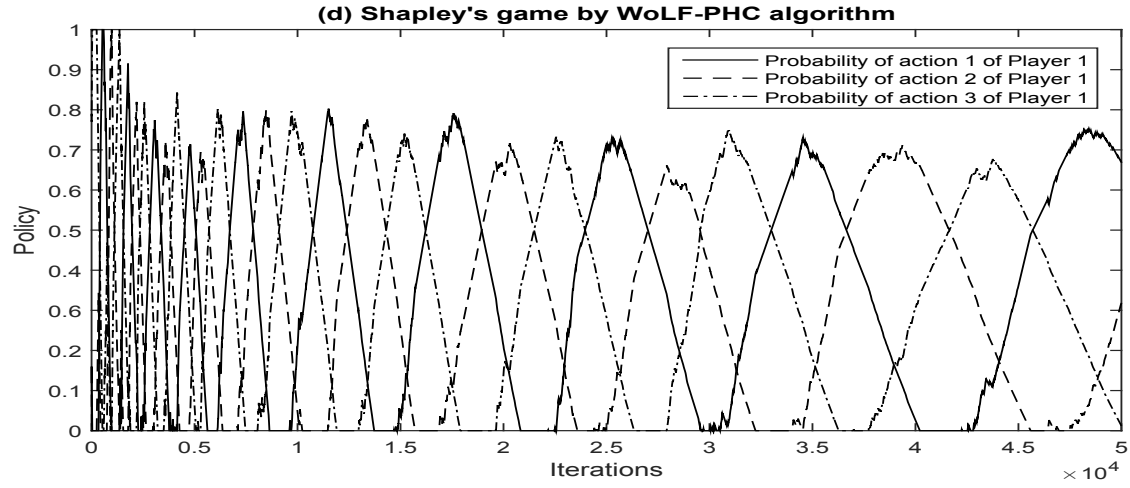

Figure 3.7: Probability of choosing Player 1's actions in the shapley's game. The proposed EMAQL, the PGA-APP, the WPL, and the WoLF-PHC algorithms are shown. 

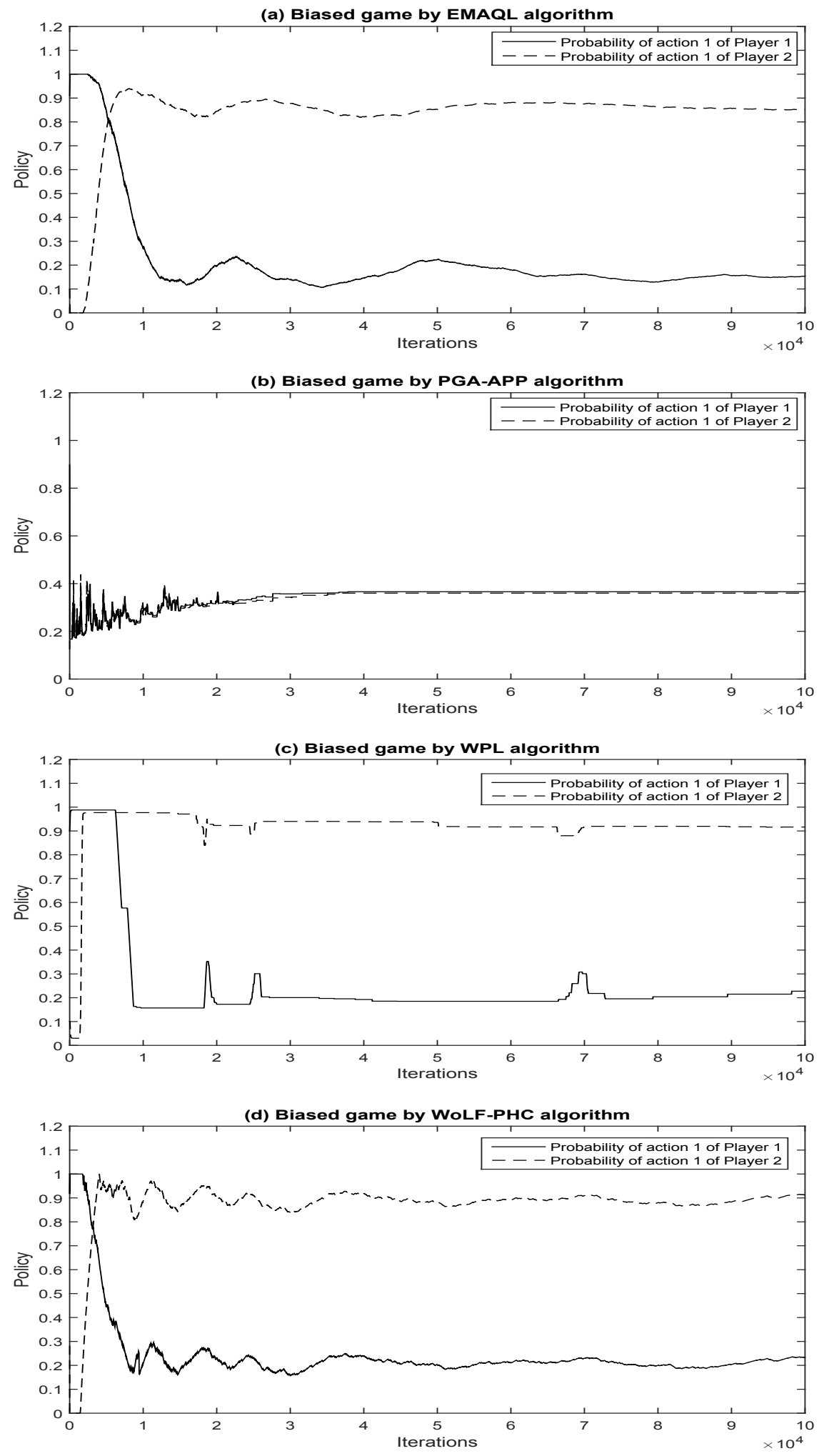

Figure 3.8: Probability of choosing the first action for both players in the biased game. The proposed EMAQL, the PGA-APP, the WPL, and the WoLF-PHC algorithms are shown. 


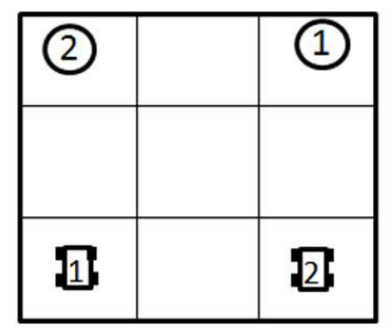

(a) Grid Game 1

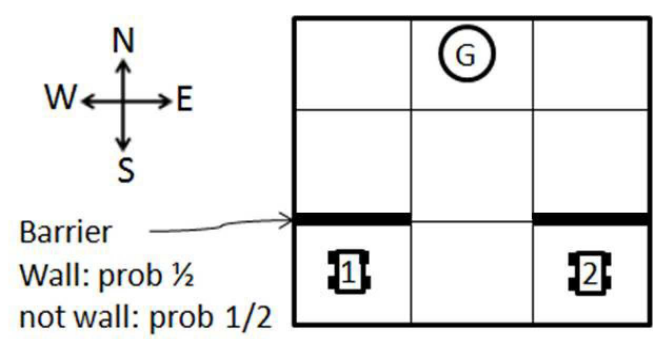

(b) Grid game 2

Figure 3.9: Two stochastic games [16].

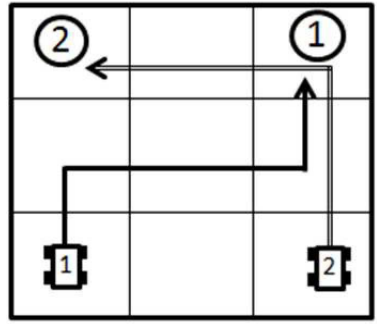

(a)

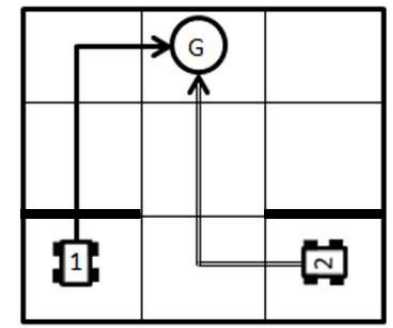

(b)

Figure 3.10: (a) A Nash equilibrium of grid game 1. (b) A Nash equilibrium of grid game $2[16]$.

converge to Nash equilibria shown in Fig. (3.10) corresponding to this stage game (the stage game at the initial state). Therefore, the figures that will be presented in this subsection will only represent the probabilities of players' actions at the initial state.

\section{Grid Game 1}

The proposed EMAQL, the WoLF-PHC, the PGA-APP and the WPL algorithms are used to learn grid game 1 depicted in Fig. (3.9)(a). Grid game 1 has ten different Nash equilibria [16]. One of these Nash equilibria (optimal paths) is shown in Fig. (3.10)(a). Fig. (3.10)(a) shows that the action North is the Nash equilibrium action 

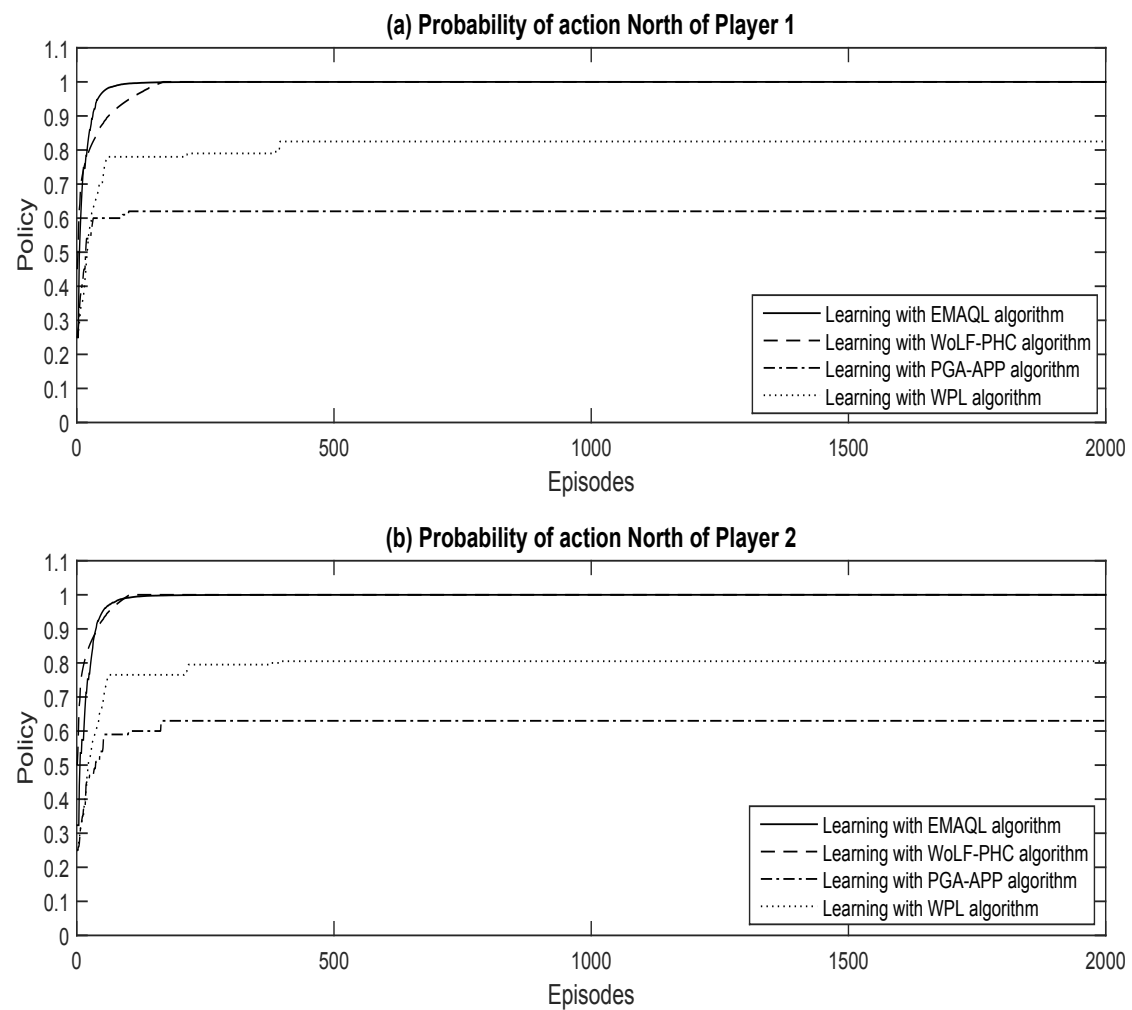

Figure 3.11: Grid game 1: (a) The probability of selecting action North by Player 1 when learning with the proposed EMAQL, the WoLF-PHC, the PGA-APP, and the WPL algorithms. (b) The probability of selecting action North by Player 2 when learning with the proposed EMAQL, the WoLF-PHC, the PGA-APP, and the WPL algorithms.

for both players when they are at their initial state. The learning parameters of the proposed EMAQL algorithm are set as follows, $\eta_{w}=\frac{1}{10+\iota / 5}, \eta_{l}^{\text {WoLF }}=2 \eta_{w}$, $\eta_{l}^{W o L S}=0.001 \eta_{w}, \zeta=0$, and $\vartheta=\frac{1}{10+0.001 \iota}$ with an exploration rate $\epsilon=0.05$, where $\iota$ is the current number of episodes. The parameters of the WoLF-PHC algorithm are set the same as those of the proposed EMAQL algorithm and with $\delta_{w}=\frac{1}{10+5 \iota}$ and $\delta_{l}=2 \delta_{w}$. The values of the parameters of the PGA-APP algorithm are the same as those of the proposed EMAQL algorithm except that $\gamma^{P}=3, \vartheta=\frac{8}{10+0.005 \iota}$, and $\eta^{P}=0.1$. On the other hand, the learning parameters of the WPL algorithm are set as follows, $\vartheta=\frac{1}{10+0.001 \iota}$ and $\eta^{W}=0.1$. 


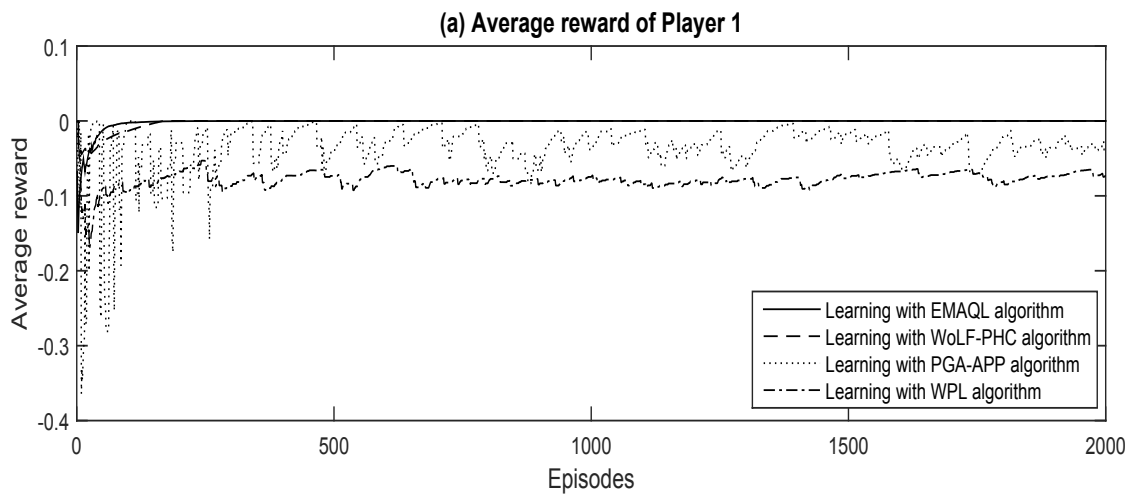

(b) Average reward of Player 2

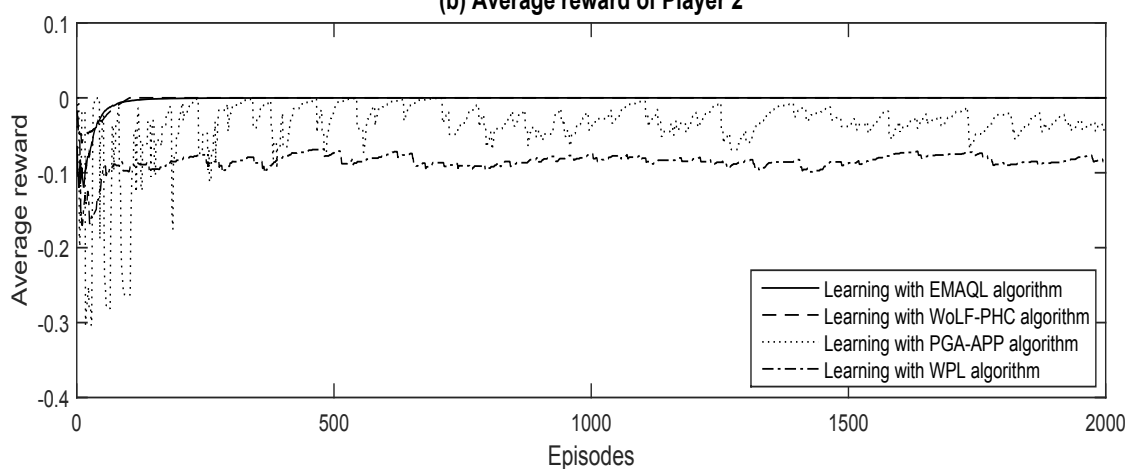

Figure 3.12: Grid game 1: (a) The average reward of Player 1 when learning with the proposed EMAQL, the WoLF-PHC, the PGA-APP, and the WPL algorithms. (b) The average reward of Player 2 when learning with the proposed EMAQL, the WoLF-PHC, the PGA-APP, and the WPL algorithms.

Fig. (3.11)(a) and Fig. (3.11)(b) show the probabilities of selecting action North by both players at their initial state when learning with the proposed EMAQL, the WoLF-PHC, the PGA-APP, and the WPL algorithms. Fig. (3.11)(a) and Fig. (3.11)(b) show that the probabilities of taking action North by both players at the initial state converge to the Nash equilibrium (converge to one) when learning with the proposed EMAQL and WoLF-PHC algorithms. However, the PGA-APP and the WPL algorithms fail to make the players' strategies converge to the Nash equilibria. Fig. (3.11) shows that the proposed EMAQL algorithm outperforms the PGA-APP and the WPL algorithms in terms of the convergence to Nash equilibrium in grid game 1. 
The maximum reward that each player can get at the initial state if both players adopt the Nash equilibrium shown in Fig (3.10)(a) is zero. Thus, the Nash equilibrium reward for both players at the initial state is zero. Fig. (3.12)(a) and Fig. (3.12)(b) show the average rewards of Player 1 and Player 2, respectively, at the initial state when learning with the proposed EMAQL, the WoLF-PHC, the PGA-APP, and the WPL algorithms. Fig. (3.12)(a) and Fig. (3.12)(b) show that the average rewards of both players at the initial state converge to the corresponding Nash equilibrium reward when only learning with the proposed EMAQL and the WoLF-PHC algorithms. On the other hand, when learning with the PGA-APP aand the WPL algorithms, the average rewards of both players converge to other values close to the Nash equilibrium reward of both players.

\section{Grid Game 2}

The proposed EMAQL, the WoLF-PHC, the PGA-APP and the WPL algorithms are also used to learn grid game 2 depicted in Fig. (3.9)(b). Grid game 2 has two Nash equilibria [16]. Fig. (3.10)(b) shows one of these Nash equilibria. As can be seen from this particular Nash equilibrium, the action East is the Nash equilibrium action for Player 1 at the initial state; whereas the action North is the Nash equilibrium action for Player 2. Thus, for the algorithms to converge to this particular Nash equilibrium at the initial state, the probability of selecting the action East by Player 1 should converge to one. The probability of selecting the action North by Player 2 at the initial state, on the other hand, should also converge to one. The learning parameters of the proposed EMAQL algorithm are set as follows, $\eta_{w}=\frac{1}{20+\iota / 5}$, $\eta_{l}^{W o L F}=2 \eta_{w}, \eta_{l}^{W o L S}=0.001 \eta_{w}, \zeta=0.25$, and $\vartheta=0.8$ with an exploration rate $\epsilon=\frac{2}{10+0.01 \iota}$, where $\iota$ is the current number of episodes. The parameters of the 

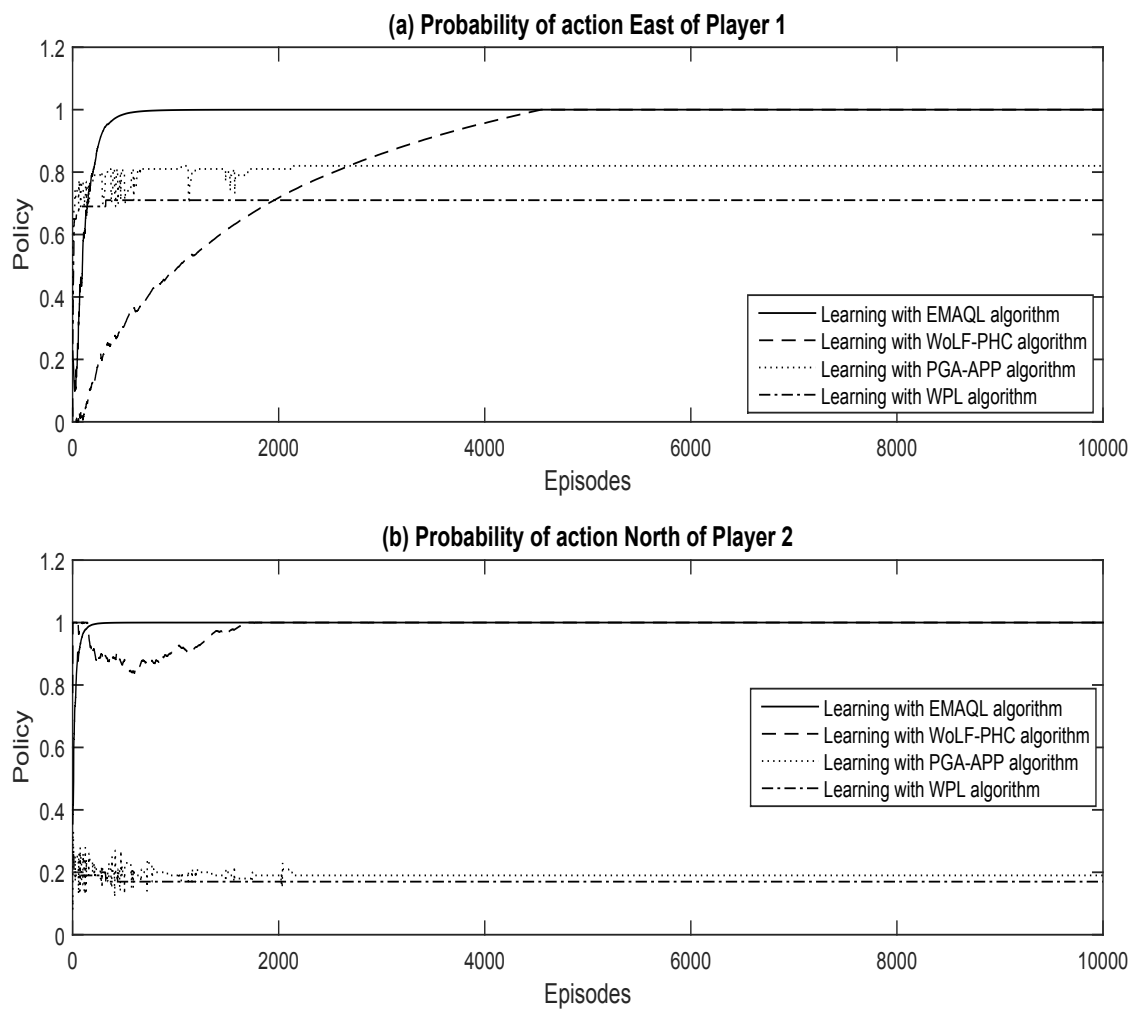

Figure 3.13: Grid game 2: (a) The probability of selecting action East by Player 1 when learning with the proposed EMAQL, the WoLF-PHC, the PGA-APP, and the WPL algorithms. (b) The probability of selecting action North by Player 2 when learning with the proposed EMAQL, the WoLF-PHC, the PGA-APP, and the WPL algorithms.

WoLF-PHC algorithm are set the same as those of the proposed EMAQL algorithm and with $\delta_{w}=\frac{1}{20+\iota}$ and $\delta_{l}=2 \delta_{w}$. The values of the parameters of the PGA-APP algorithm are the same as those of the proposed EMAQL algorithm except that $\gamma^{P}=3, \vartheta=\frac{8}{10+0.001 \iota}, \zeta=0.1$, and $\eta^{P}=0.1$. On the other hand, the learning parameters of the WPL algorithm are set as follows, $\vartheta=\frac{1}{10+0.001 \iota}, \zeta=0.1$ and $\eta^{W}=0.1$.

Fig. (3.13)(a) shows the probability of selecting action East by Player 1 at the initial state when learning with the proposed EMAQL, the WoLF-PHC, the PGA-APP, and the WPL algorithms. Fig. (3.13)(a) illustrates that the probability of selecting the action East by Player 1 at the initial state successfully converges to one (Nash 

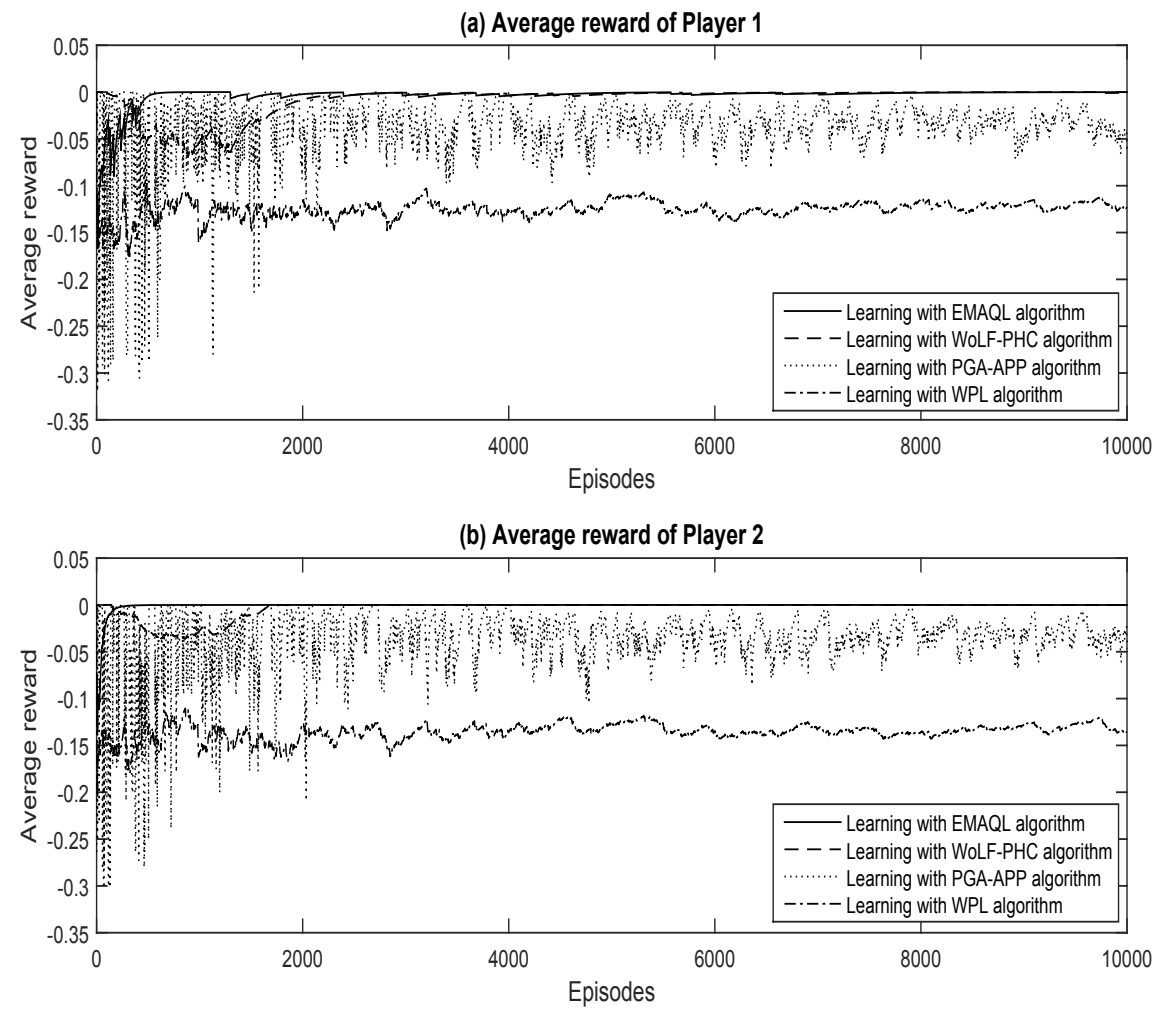

Figure 3.14: Grid game 2: (a) The average reward of Player 1 when learning with the proposed EMAQL, the WoLF-PHC, the PGA-APP, and the WPL algorithms. (b) The average reward of Player 2 when learning with the proposed EMAQL, the WoLF-PHC, the PGA-APP, and the WPL algorithms.

equilibrium) when learning with the proposed EMAQL and WoLF-PHC algorithms. However, the PGA-APP and the WPL algorithms fail to make Player 1 choose the action East with a probability of one at the initial state. Fig. (3.13)(b) shows the probability of selecting action North by Player 2 at the initial state when learning with the proposed EMAQL, the WoLF-PHC, the PGA-APP, and the WPL algorithms. As can be seen from Fig. (3.13)(b), the probability of selecting action North by Player 2 at the initial state successfully converges to one (Nash equilibrium) when Player 2 learns its strategy with the proposed EMAQL and the WoLF-PHC algorithms. The PGA-APP and the WPL algorithms, on the other hand, fail to make Player 2 choose action North with a probability of one at the initial state. Fig. 
(3.13) shows that the proposed EMAQL algorithm outperforms the PGA-APP and the WPL algorithms in terms of the convergence to Nash equilibrium in grid game 2.

In grid game 2, the Nash equilibrium reward for both players at the initial state is zero. Fig. (3.14)(a) and Fig. (3.14)(b) show the average rewards of Player 1 and Player 2 at the initial state, respectively, when learning with the proposed EMAQL, the WoLF-PHC, the PGA-APP, and the WPL algorithms. Fig. (3.14)(a) and Fig. (3.14)(b) show that the average rewards of both players at the initial state converge to the corresponding Nash equilibrium reward when only learning with the proposed EMAQL and the WoLF-PHC algorithms. On the other hand, when learning with the PGA-APP aand the WPL algorithms, the average rewards of both players converge to other values close to the Nash equilibrium reward of both players at the initial state.

\subsection{Summary and Discussion}

New multi-agent policy iteration learning algorithms are proposed in this work; the CLR-EMAQL algorithm and the EMAQL algorithm. The proposed algorithms use the exponential moving average (EMA) estimation technique along with the Qlearning algorithm to update the learning agent's policy. The algorithms are proposed to solve the problem of learning in matrix and stochastic games when:

- each learning agent does not have enough information about the underlying game (i.e. each learning agent does not know the Nash equilibrium of the game and/or does not know its reward function);

- each learning agent is oblivious to the other agents. That is,

- each learning agent does not share information with the other learning 
agents;

- each learning agent does not know the other learning agents' immediate rewards and actions in case of matrix games, and does not know the other learning agents' immediate rewards in case of stochastic games.

The proposed CLR-EMAQL algorithm uses one constant learning rate $\left(\eta_{c}\right)$ when updating the learning agent's strategy. The proposed CLR-EMAQL algorithm succeeds to converge to Nash equilibrium only when the game has a pure Nash equilibrium. A theoretical analysis that shows the convergence of the proposed CLR-EMAQL algorithm to Nash equilibrium in games with pure Nash equilibrium is provided in this article. On the other hand, the proposed EMAQL algorithm uses two different decaying learning rates $\left(\eta_{w}\right.$ and $\left.\eta_{l}\right)$ when updating the agent's strategy. The values of these variable learning rates are inversely proportional to the number of iterations (or episodes) and are set based on one of two different mechanisms; the Win-or-LearnFast (WoLF) mechanism or the Win-or-Learn-Slow (WoLS) mechanism. The WoLF mechanism makes the proposed EMAQL algorithm learn fast when the algorithm is losing and learn cautiously when the algorithm is winning. On the other hand, the WoLS mechanism makes the proposed EMAQL algorithm learn fast when the algorithm is winning and learn cautiously when the algorithm is losing. The proposed EMAQL algorithm uses the rewards received by the learning agent to decide which mechanism (WoLF mechanism or WoLS mechanism) to use for the game being learned. The proposed EMAQL algorithm succeeds to converge to Nash equilibrium in games with pure or mixed Nash equilibrium. A theoretical analysis that shows the convergence of the proposed EMAQL algorithm to Nash equilibrium in games with pure Nash equilibria is provided. In the case of games with mixed Nash equilibrium, our mathematical analysis shows that the proposed EMAQL algorithm converges to an equilibrium. Although our mathematical analysis does not explicitly show that the 
proposed EMAQL algorithm converges to a Nash equilibrium, our simulation results indicate that the proposed EMAQL algorithm does converge to Nash equilibrium. To verify our theoretical analysis, the proposed EMAQL algorithm is applied to a variety of matrix and stochastic games. Our simulation results are shown to be consistent with our theoretical analysis. The results also show that the proposed EMAQL algorithm converges to Nash equilibrium at a variety of matrix and stochastic games at which the PGA-APP, the WPL, and the WoLF-PHC algorithms fail to converge to Nash equilibrium. This makes the proposed EMAQL algorithm outperform the PGA-APP, the WPL, and the WoLF-PHC algorithms in terms of the convergence to Nash equilibrium. 


\section{Chapter 4}

\section{Reinforcement Learning in Differential}

\section{Games}

\subsection{Introduction}

Fuzzy logic controllers (FLCs) have recently received considerable attention as intelligent controllers, and are used in engineering applications [93,94]. FLCs have been widely used to deal with plants that are nonlinear and ill-defined $[95,96,140]$. They can also deal with plants with high uncertainty in the knowledge about their environments $[97,98]$. Several learning approaches have been developed to tune FLCs so that the desired performance is achieved. Some of these approaches design the fuzzy systems from input-output data by using different mechanisms such as a table look-up approach, a genetic algorithm approach, a gradient-descent training approach, a recursive least squares approach, and clustering [121,141]. This type of learning is called supervised learning where a training data set is used to learn from. However, in this type of learning, the performance of the trained FLC will depend on the performance of the expert. In addition, the training data set used in supervised learning may be hard or expensive to obtain. In such cases, we think of alternative techniques where neither a priori knowledge nor a training data set 
is required. In this case, reward-based learning techniques, such as reinforcement learning algorithms, can be used. The main advantage of such reinforcement learning algorithms is that they do not need either a known model for the process nor an expert to learn from [128].

Reinforcement learning (RL) is a learning technique that maps situations to actions so that an agent learns from the experience of interacting with its environment $[20,114]$. Reinforcement learning has attracted attention and been widely used in intelligent robot control systems $[3,101,103,104,115-118,126]$. Reinforcement learning has also been used for solving nonlinear optimal control problems [88-92,106-112]. Without knowing which actions to take, the reinforcement learning agent exploits and explores actions to discover which action gives the maximum reward in the long run. Different from supervised learning, which is learning from input-output data provided by an expert, reinforcement learning is adequate for learning from interaction by using very simple "evaluative" or "critic" information instead of "instructive" information [20]. Most of the traditional reinforcement learning algorithms represent the state/stateaction value function as a look-up table for each state/state-action-pair [20]. Despite the theoretical foundations of these algorithms and their effectiveness in many applications, these reinforcement learning approaches cannot be applied to real applications with large state and action spaces $[3,17,20,119,124,125]$. This is because of the phenomenon known as the curse of dimensionality caused by the exponentially grown number of states when the number of state variables increases [20]. Moreover, traditional reinforcement learning approaches cannot be applied to differential games, where the states and actions are continuous. One of the possible solutions to the problem of continuous domains is to discretize the state and action spaces. However, this discretization may also lead to the problem of the curse of dimensionality that appears when discretizing large continuous states and/or actions $[3,20,126]$. To 
overcome these issues that lead to the problem of the curse of dimensionality, one may use a function approximation system (FAS) to represent the large discrete and/or continuous spaces $[20,99,123,141]$. Different types of FASs are used in the literature, and the gradient-descent-based FASs are among the most widely used ones [20]. In addition, the gradient-descent-based FASs are well suited to online reinforcement learning [20].

Several fuzzy reinforcement learning approaches have been proposed in the literature to deal with differential games (that have continuous state and action spaces) by using gradient-descent-based FASs $[48,99,100,103,126,127]$. Some of these approaches only tune the output parameters of their FASs $[48,99,103]$, where the input parameters of their FASs are kept fixed. On the other hand, the other approaches tune both the input and the output parameters of their FASs $[100,126,127]$.

In [99], the author proposed a fuzzy actor critic learning (FACL) algorithm that uses gradient-descent-based fuzzy inference systems (FISs) as FASs. The FACL algorithm only tunes the output parameters (the consequent parameters) of its FISs (the actor and the critics) by using the temporal difference error (TD) calculated based on the state value function. However, the input parameters (the premise parameters) of its FISs are kept fixed during the learning process. In [48], the authors proposed a fuzzy reinforcement learning approach that uses gradient-descent-based FISs as FASs. Their approach only tunes the output parameters of the FASs based on the TD error calculated based on the state value functions of the two successive states in the state transition. In [103], the authors proposed the generalized probabilistic fuzzy reinforcement learning (GPFRL) algorithm, which is a modified version of the actor-critic learning architecture. The GPFRL algorithm uses gradient-descent-based FISs as FASs. The GPFRL only tunes the output parameters of its FASs based on 
the TD error of the critic and the performance function of the actor. In [126], the authors proposed a fuzzy learning approach that uses a time delay neural network (TDNN) and a FIS as gradient-descent-based FASs. Their approach tunes the input and the output parameters of the FASs based on the TD error calculated based on the state-action value function. In [127], the authors proposed a fuzzy actor-critic reinforcement learning network (FACRLN) algorithm based on a fuzzy radial basis function (FRBF) neural network. The FACRLN uses the FRBF neural networks as FASs. The FACRLN algorithm tunes the input and the output parameters of the FASs based on the TD error calculated by the temporal difference of the value function between the two successive states in the state transition. In [100], the authors proposed the Q-learning fuzzy inference system (QLFIS) algorithm that uses gradient-descent-based FISs as FASs. The QLFIS algorithm tunes the input and the output parameters of the FASs based on the TD error of the state-action value functions of the two successive states in the state transition. However, all these fuzzy reinforcement learning algorithms $[48,99,100,103,126,127]$ use what so called "direct algorithms described in [113]" to tune the parameters of their FASs. Although direct algorithms have been widely used in the tunning mechanism of the parameters for the FASs, the direct algorithms may lead the FASs to unpredictable results and, in some cases, to divergence [113,129-132].

In this chapter, we propose a new fuzzy reinforcement learning algorithm for differential games, where the states and actions are continuous. The proposed algorithm is published in $[101,102]$. The proposed algorithm uses FASs whose parameters are updated differently from the updating mechanisms used in the algorithms proposed in $[48,99,100,103,126,127]$. Unlike the algorithms presented in the literature which use the direct algorithms to update their FASs, the proposed algorithm uses the residual gradient value iteration algorithm proposed in [113] to tune the input and 
the output parameters of its FASs. The direct algorithms may not converge to an answer in some cases, while the residual gradient algorithms are always guaranteed to converge to a local minimum [113, 129-132].

We investigate the proposed algorithm on different pursuit-evasion differential games because this kind of games is considered as a general problem for several other problems such as the problems of wall following, obstacle avoidance, and path planning. Moreover, pursuit-evasion games are useful for many real-world applications such as search and rescue, locating and capturing hostile intruders, localizing and neutralizing environmental threats, and surveillance and tracking $[3,100]$. The proposed algorithm is used to learn three different pursuit-evasion differential games. In the first game, the evader is following a simple control strategy, whereas the pursuer is learning its control strategy to capture the evader in minimum time. In the second game, it is also only the pursuer that is learning. However, the evader is following an intelligent control strategy that exploits the advantage of the maneuverability of the evader. In the third game, we make both the pursuer and the evader learn their control strategies. Therefore, the complexity of the system will increase as the learning in a multi-robot system is considered as a problem of a "moving target" [9]. In the multi-robot system learning, each robot will try to learn its control strategy by interacting with the other robot which is also learning its control strategy at the same time. Thus, the best-response policy of each learning robot may keep changing during learning in a multi-robot system. The proposed algorithm outperforms the FACL and QLFIS algorithms proposed in [99] and [100] in terms of convergence and speed of learning when they all are used to learn the pursuit-evasion differential games considered in this chapter. 


\subsection{Direct and Residual Gradient Algorithms}

Traditional reinforcement learning algorithms such as the Q-learning algorithm and the value iteration algorithm represent the value functions as lookup tables and are guaranteed to converge to optimal values [20]. These algorithms have to be combined with function-approximation systems when they are applied to real applications with large state and action spaces or with continuous state and action spaces. The direct and the residual gradient algorithms described in [113] can be used when the traditional Q-learning and the value iteration algorithms are combined with function-approximation systems. In [113], the author illustrated with some examples that the direct algorithms may converge fast but may become unstable in some cases. In addition, the author showed that the residual gradient algorithms converge in those examples and are always guaranteed to converge to a local minimum. Another study presented in [131] shows that the direct algorithms are faster than the residual gradient algorithms only when the value function is represented in a tabular form. However, when FASs are involved, the direct algorithms are not always faster than the residual gradient algorithms. In other words, when FASs are involved, the residual gradient algorithms are considered to be the superior algorithms as they are always guaranteed to converge, whereas the direct algorithms may not converge to an answer in some cases. Other different studies [129,130,132] confirm the results presented in [113] in terms of the superiority of the residual gradient algorithms as they are always shown to converge. To illustrate more the difference between the direct algorithms and the residual gradient algorithms, we will give two different examples of these algorithms; the direct value iteration algorithm (an example of the direct algorithms) and the residual gradient value iteration algorithm (an example of the residual gradient algorithms). 


\subsubsection{The Direct Value Iteration Algorithm}

For Markov decision processes (MDPs), the value function $V_{t}\left(\mathbf{s}_{t}\right)$ at the state $\mathbf{s}_{t}$ for approximation of the reinforcement rewards can be defined as follows [48, 122],

$$
V_{t}\left(\mathbf{s}_{t}\right)=E\left\{\sum_{\kappa=t}^{\infty} \zeta^{\kappa-t} r_{\kappa}\right\},
$$

where $\zeta \in[0,1)$ is a discount factor and $r_{\kappa}$ is the immediate external reward that the learning agent gets from the learning environment.

The recursive form of Eq. (4.1) can be defined as follows [48, 122],

$$
V_{t}\left(\mathbf{s}_{t}\right)=r_{t}+\zeta V_{t}\left(\mathbf{s}_{t+1}\right)
$$

The temporal difference residual error, $\Delta_{t}$, and the mean square error, $E_{t}$, between the two sides of Eq. (4.2) are given as follows,

$$
\begin{gathered}
\left.\Delta_{t}=\left[r_{t}+\zeta V_{t}\left(\mathbf{s}_{t+1}\right)\right]-V_{t}\left(\mathbf{s}_{t}\right)\right], \\
E_{t}=\frac{1}{2} \Delta_{t}^{2} .
\end{gathered}
$$

For a deterministic MDP, after transition from a state to another, the direct value iteration algorithm updates the weights of the function-approximation system as follows [113],

$$
\psi_{t+1}=\psi_{t}-\rho \frac{\partial E_{t}}{\partial \psi_{t}}
$$

where $\psi_{t}$ represents the input and output parameters of the function-approximation

system that need to be tuned, $\rho$ is a learning rate, and the term $\frac{\partial E_{t}}{\partial \psi_{t}}$ is defined as 
follows,

$$
\frac{\partial E_{t}}{\partial \psi_{t}}=\Delta_{t} \frac{\partial \Delta_{t}}{\partial \psi_{t}}=-\left[r_{t}+\zeta V_{t}\left(\mathbf{s}_{t+1}\right)-V_{t}\left(\mathbf{s}_{t}\right)\right] \cdot \frac{\partial}{\partial \psi_{t}} V_{t}\left(\mathbf{s}_{t}\right)
$$

Thus,

$$
\psi_{t+1}=\psi_{t}+\rho\left[r_{t}+\zeta V_{t}\left(\mathbf{s}_{t+1}\right)-V_{t}\left(\mathbf{s}_{t}\right)\right] \cdot \frac{\partial}{\partial \psi_{t}} V_{t}\left(\mathbf{s}_{t}\right)
$$

The direct value iteration algorithm updates the derivative $\frac{\partial E_{t}}{\partial \psi_{t}}$ as in Eq. (4.6). This equation shows that the direct value iteration algorithm treats the value function $V_{t}\left(\mathbf{s}_{t+1}\right)$ in the temporal error $\Delta_{t}$ as a constant. Therefore, the derivative $\frac{\partial V_{t}\left(\mathbf{s}_{t+1}\right)}{\partial \psi_{t}}$ will be zero. However, the value function $V_{t}\left(\mathbf{s}_{t+1}\right)$ is not a constant, and it is a function of the input and output parameters of the function-approximation system,

$\psi_{t}$. Therefore, the derivative $\frac{\partial V_{t}\left(\mathbf{s}_{t+1}\right)}{\partial \psi_{t}}$ should not be assigned to zero during the tuning of the input and the output parameters of the function-approximation system.

\subsubsection{The Residual Gradient Value Iteration Algorithm}

The residual gradient value iteration algorithm updates the weights of the functionapproximation system as follows [113],

$$
\psi_{t+1}=\psi_{t}-\rho \frac{\partial E_{t}}{\partial \psi_{t}}
$$

where,

$$
\frac{\partial E_{t}}{\partial \psi_{t}}=\Delta_{t} \frac{\partial \Delta_{t}}{\partial \psi_{t}}=\left[r_{t}+\zeta V_{t}\left(\mathbf{s}_{t+1}\right)-V_{t}\left(\mathbf{s}_{t}\right)\right] \cdot\left[\frac{\partial}{\partial \psi_{t}} \zeta V_{t}\left(\mathbf{s}_{t+1}\right)-\frac{\partial}{\partial \psi_{t}} V_{t}\left(\mathbf{s}_{t}\right)\right]
$$

Thus, 


$$
\psi_{t+1}=\psi_{t}-\rho\left[r_{t}+\zeta V_{t}\left(\mathbf{s}_{t+1}\right)-V_{t}\left(\mathbf{s}_{t}\right)\right] \cdot\left[\frac{\partial}{\partial \psi_{t}} \zeta V_{t}\left(\mathbf{s}_{t+1}\right)-\frac{\partial}{\partial \psi_{t}} V_{t}\left(\mathbf{s}_{t}\right)\right]
$$

The residual gradient value iteration algorithm updates the derivative $\frac{\partial E_{t}}{\partial \psi_{t}}$ as in Eq. (4.9). In this equation, the residual gradient value iteration algorithm treats the value function $V_{t}\left(\mathbf{s}_{t+1}\right)$ in the temporal error $\Delta_{t}$ as a function of the input and output

parameters of the FAS, $\psi_{t}$. Therefore, the derivative $\frac{\partial V_{t}\left(\mathbf{s}_{t+1}\right)}{\partial \psi_{t}}$ will not be assigned to zero during the tuning of the input and output parameters of the FAS. This will make the residual gradient value iteration algorithm performs better than the direct value iteration algorithm in terms of convergence [113].

\subsection{Fuzzy Inference Systems}

Reinforcement learning approaches have recently been applied to differential games, including the pursuit-evasion games $[48,120,121]$. Most of traditional reinforcement learning algorithms represent the state/state-action value function as a look-up table for each state/state-action-pair [20]. Despite the theoretical foundations of these algorithms and their effectiveness in many applications, these approaches cannot be applied to real applications with continuous domains $[20,119,124,125]$. To solve this problem, one may use fuzzy inference systems (as FASs) to represent the continuous space $[20,99,141]$.

Among the most widely used FISs are the Mamdani FIS proposed in [145] and the Takagi-Sugeno-Kang (TSK) FIS proposed in [146,147]. The FISs used in this chapter are zero-order TSK FISs with constant consequents. Each FIS consists of $L$ rules. The inputs of each rule are $n$ state variables; whereas the consequent of each rule is 
a constant number. Each rule $l(l=1, \ldots, L)$ has the following form,

$$
R_{l}: \text { IF } s_{1} \text { is } A_{1}^{l}, \ldots \text {, and } s_{n} \text { is } A_{n}^{l} \quad \text { THEN } \quad z_{l}=k_{l} \text {, }
$$

where $s_{\imath},(\imath=1, \ldots, n)$, is the $\imath$ th input state variable of the fuzzy system, $n$ is the number of input state variables, and $A_{\imath}^{l}$ is the linguistic value of the input $s_{\imath}$ at the rule $l$. Each input $s_{\imath}$ has $h$ membership functions. The variable $z_{l}$ represents the output variable of the rule $l$, and $k_{l}$ is a constant that describes the consequent parameter of the rule $l$. In this work, Gaussian membership functions are used for the inputs and each membership function (MF) is defined as follows,

$$
\mu^{A_{\imath}^{l}}\left(s_{\imath}\right)=\exp \left(-\left(\frac{s_{\imath}-m}{\sigma}\right)^{2}\right)
$$

where $\sigma$ and $m$ are the standard deviation and the mean, respectively.

In each FIS used in this chapter, the total number of the standard deviations of the membership functions of its inputs is defined as $H$, where $H=n \times h$. In addition, the total number of the means of the membership functions of its inputs is $H$. Thus, for each FIS used in this chapter, the standard deviations and the means of the membership functions of the inputs are defined, respectively, as $\sigma_{\jmath}$ and $m_{\jmath}$, where $\jmath=1, \ldots, H$. We define the set of the parameters of the membership functions of each input, $\Omega\left(s_{\imath}\right)$, as follows, 


$$
\begin{aligned}
& \Omega\left(s_{1}\right)=\left\{\left(\sigma_{1}, m_{1}\right),\left(\sigma_{2}, m_{2}\right), \ldots \ldots \ldots \ldots \ldots .,\left(\sigma_{h}, m_{h}\right)\right\}, \\
& \Omega\left(s_{2}\right)=\left\{\left(\sigma_{h+1}, m_{h+1}\right),\left(\sigma_{h+2}, m_{h+2}\right), \ldots \ldots \ldots,\left(\sigma_{2 h}, m_{2 h}\right)\right\},
\end{aligned}
$$

$$
\Omega\left(s_{n}\right)=\left\{\left(\sigma_{(n-1) h+1}, m_{(n-1) h+1}\right),\left(\sigma_{(n-1) h+2}, m_{(n-1) h+2}\right), \ldots,\left(\sigma_{H}, m_{H}\right)\right\} .
$$

The output of the fuzzy system is given by the following equation when we use the product inference engine with singleton fuzzifier and center-average (weighted average) defuzzifier [141],

$$
Z\left(\mathbf{s}_{t}\right)=\frac{\sum_{l=1}^{L}\left[\left(\prod_{l=1}^{n} \mu^{A_{\imath}^{l}}\left(s_{\imath}\right)\right) k_{l}\right]}{\sum_{l=1}^{L}\left(\prod_{\imath=1}^{n} \mu^{A_{\imath}^{l}}\left(s_{\imath}\right)\right)}=\sum_{l=1}^{L} \Phi_{l}\left(\mathbf{s}_{t}\right) k_{l}
$$

where $\mathbf{s}_{t}=\left(s_{1}, \ldots, s_{n}\right)$ is the state vector, $\mu^{A_{\imath}^{l}}$ describes the membership value of the input state variable $s_{\imath}$ in the rule $l$, and $\Phi_{l}\left(\mathbf{s}_{t}\right)$ is the normalized activation degree (normalized firing strength) of the rule $l$ at the state $\mathbf{s}_{t}$ and is defined as follows:

$$
\Phi_{l}\left(\mathbf{s}_{t}\right)=\frac{\prod_{\imath=1}^{n} \mu^{A_{\imath}^{l}}\left(s_{\imath}\right)}{\sum_{l=1}^{L}\left(\prod_{\imath=1}^{n} \mu^{A_{\imath}^{l}}\left(s_{\imath}\right)\right)}=\frac{\omega_{l}\left(\mathbf{s}_{t}\right)}{\sum_{l=1}^{L} \omega_{l}\left(\mathbf{s}_{t}\right)}
$$

where $\omega_{l}\left(\mathbf{s}_{t}\right)$ is the firing strength of the rule $l$ at the state $\mathbf{s}_{t}$, and it is defined as follows, 


$$
\omega_{l}\left(\mathbf{s}_{t}\right)=\prod_{\imath=1}^{n} \mu^{A_{\imath}^{l}}\left(s_{\imath}\right) .
$$

We define the set of the parameters of each firing strength of each rule in each FIS, $\Omega\left(\omega_{l}\right)$, as follows,

$$
\begin{gathered}
\Omega\left(\omega_{1}\right)=\left\{\left(\sigma_{1}, m_{1}\right),\left(\sigma_{h+1}, m_{h+1}\right), \ldots,\left(\sigma_{(n-1) h+1}, m_{(n-1) h+1}\right)\right\} \\
\Omega\left(\omega_{2}\right)=\left\{\left(\sigma_{1}, m_{1}\right),\left(\sigma_{h+1}, m_{h+1}\right), \ldots,\left(\sigma_{(n-1) h+2}, m_{(n-1) h+2}\right)\right\}, \\
\cdot \\
\Omega\left(\omega_{h}\right)=\left\{\left(\sigma_{1}, m_{1}\right),\left(\sigma_{h+1}, m_{h+1}\right), \ldots,\left(\sigma_{H}, m_{H}\right)\right\}, \\
\Omega\left(\omega_{h+1}\right)=\left\{\left(\sigma_{1}, m_{1}\right),\left(\sigma_{h+2}, m_{h+2}\right), \ldots,\left(\sigma_{(n-1) h+1}, m_{(n-1) h+1}\right)\right\}, \\
\Omega\left(\omega_{L}\right)=\left\{\left(\sigma_{h}, m_{h}\right),\left(\sigma_{2 h}, m_{2 h}\right), \ldots,\left(\sigma_{H}, m_{H}\right)\right\} . \\
.
\end{gathered}
$$




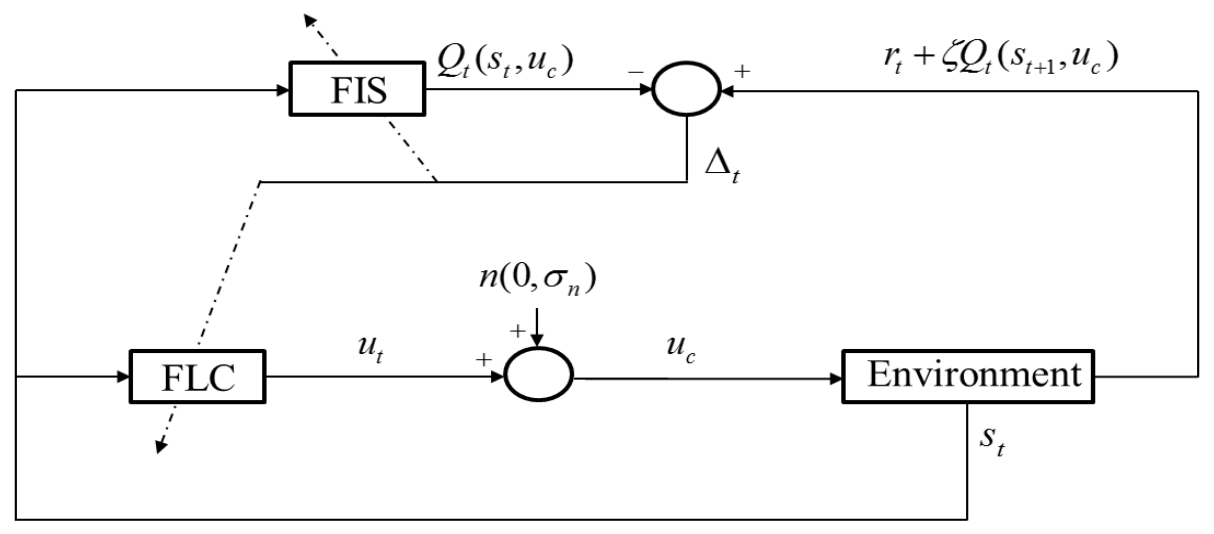

Figure 4.1: The QLFIS technique [100].

\subsection{The Q-learning Fuzzy Inference System (QL- FIS) Algorithm}

The Q-learning Fuzzy Inference System (QLFIS) algorithm is a modified version of the algorithms presented in [48] and [126]. The QLFIS algorithm combines the Q-learning algorithm with a FAS, and is applied directly to games with continuous state and action spaces. The structure of the QLFIS algorithm is shown in Fig. 4.1. The QLFIS algorithm uses a FAS (FIS) to estimate the state-action value functions $Q\left(\mathbf{s}_{t}, u\right)$. The QLFIS algorithm also uses another FAS (FLC) to generate the continuous action. The QLFIS algorithm tunes the input and the output parameters of its FASs [100]. The QLFIS algorithm uses the so-called direct algorithms described in [113] as a mechanism to tune the input and the output parameters of its FASs.

\subsubsection{Update Rules for the FIS}

The input parameters of the FIS are the parameters of the Gaussian membership functions of its input; the standard deviations $\sigma_{\jmath}$ and the means $m_{\jmath}$. On the other hand, the output parameters of the FIS are the consequent (or conclusion) parts 
of the fuzzy rules, $k_{l}$. To simplify notations, we refer to the input and the output parameters of the FIS as $\psi^{Q}$. Thus, the update rules of the input and the output parameters for the FIS of the QLFIS algorithm are given as follows [100],

$$
\psi_{t+1}^{Q}=\psi_{t}^{Q}+\rho^{Q} \Delta_{t} \frac{\partial Q_{t}\left(\mathbf{s}_{t}, u_{c}\right)}{\partial \psi_{t}^{Q}},
$$

where $\rho^{Q}$ is a learning rate for the FIS parameters, and $\Delta_{t}$ is the temporal difference error that is defined as follows,

$$
\Delta_{t}=r_{t}+\zeta Q_{t}\left(\mathbf{s}_{t+1}, u_{c}\right)-Q_{t}\left(\mathbf{s}_{t}, u_{c}\right),
$$

where $r_{t}$ is the reward received at time $t, \zeta$ is a discount factor, and $Q_{t}\left(\mathbf{s}_{t}, u_{c}\right)$ is the estimated state-action value function at the state $\mathbf{s}_{t}$.

The term $\frac{\partial Q_{t}\left(\mathbf{s}_{t}, u_{c}\right)}{\partial \psi_{t}^{Q}}$ in Eq. (4.18) is computed as in [100] as follows,

$$
\begin{gathered}
\frac{\partial Q_{t}\left(\mathbf{s}_{t}, u_{c}\right)}{\partial k_{l}}=\Phi_{l}\left(\mathbf{s}_{t}\right) \\
\frac{\partial Q_{t}\left(\mathbf{s}_{t}, u_{c}\right)}{\partial \sigma_{\jmath}}=\frac{k_{l}-Q_{t}\left(\mathbf{s}_{t}, u_{c}\right)}{\sum_{l} \omega_{l}\left(\mathbf{s}_{t}\right)} \omega_{l}\left(\mathbf{s}_{t}\right) \frac{2\left(s_{\imath}-m_{\jmath}\right)^{2}}{\left(\sigma_{\jmath}\right)^{3}} \\
\frac{\partial Q_{t}\left(\mathbf{s}_{t}, u_{c}\right)}{\partial m_{\jmath}}=\frac{k_{l}-Q_{t}\left(\mathbf{s}_{t}, u_{c}\right)}{\sum_{l} \omega_{l}\left(\mathbf{s}_{t}\right)} \omega_{l}\left(\mathbf{s}_{t}\right) \frac{2\left(s_{\imath}-m_{\jmath}\right)}{\left(\sigma_{\jmath}\right)^{2}}
\end{gathered}
$$

\subsubsection{Update Rules for the FLC}

The input parameters of the FLC are the parameters of the Gaussian membership functions of its input; the standard deviations $\sigma_{\jmath}$ and the means $m_{\jmath}$. On the other hand, the output parameters of the FLC are the consequent (or conclusion) parts of the fuzzy rules, $k_{l}$. We refer to the input and the output parameters of the FLC as 
$\psi^{u}$. Thus, the update rules of the input and the output parameters for the FLC of the QLFIS algorithm are given as follows [100],

$$
\psi_{t+1}^{u}=\psi_{t}^{u}+\rho^{u} \Delta_{t} \frac{\partial u_{t}}{\partial \psi_{u_{t}}}\left[\frac{u_{c}-u_{t}}{\sigma_{n}}\right]
$$

where $\rho^{u}$ is a learning rate for the FLC parameters, $u_{c}$ is the output of the FLC with a random Gaussian noise. The term $\frac{\partial u_{t}}{\partial \psi_{t}^{u}}$ in Eq. (4.23) can be calculated by replacing $Q_{t}\left(\mathbf{s}_{t}, u_{c}\right)$ with $u_{t}$ in Eq. (4.20), Eq. (4.21) and Eq. (4.22) as follows,

$$
\begin{gathered}
\frac{\partial u_{t}}{\partial k_{l}}=\Phi_{l}\left(\mathbf{s}_{t}\right) \\
\frac{\partial u_{t}}{\partial \sigma_{\jmath}}=\frac{k_{l}-u_{t}}{\sum_{l} \omega_{l}\left(\mathbf{s}_{t}\right)} \omega_{l}\left(\mathbf{s}_{t}\right) \frac{2\left(s_{\imath}-m_{\jmath}\right)^{2}}{\left(\sigma_{\jmath}\right)^{3}}, \\
\frac{\partial u_{t}}{\partial m_{\jmath}}=\frac{k_{l}-u_{t}}{\sum_{l} \omega_{l}\left(\mathbf{s}_{t}\right)} \omega_{l}\left(\mathbf{s}_{t}\right) \frac{2\left(s_{\imath}-m_{\jmath}\right)}{\left(\sigma_{\jmath}\right)^{2}} .
\end{gathered}
$$

\subsection{The Pursuit-Evasion Game}

A variety of pursuit-evasion games have been studied in different contexts [52-71]. The pursuit-evasion game is defined as a differential game [133]. In this game, the pursuer's objective is to capture the evader, whereas the evader's objective is to escape from the pursuer or at least prolong the capture time. Fig. (4.2) shows the model of the pursuit-evasion differential game. The equations of motion of the pursuer and the evader robots can be described by the following equations [134,135], 


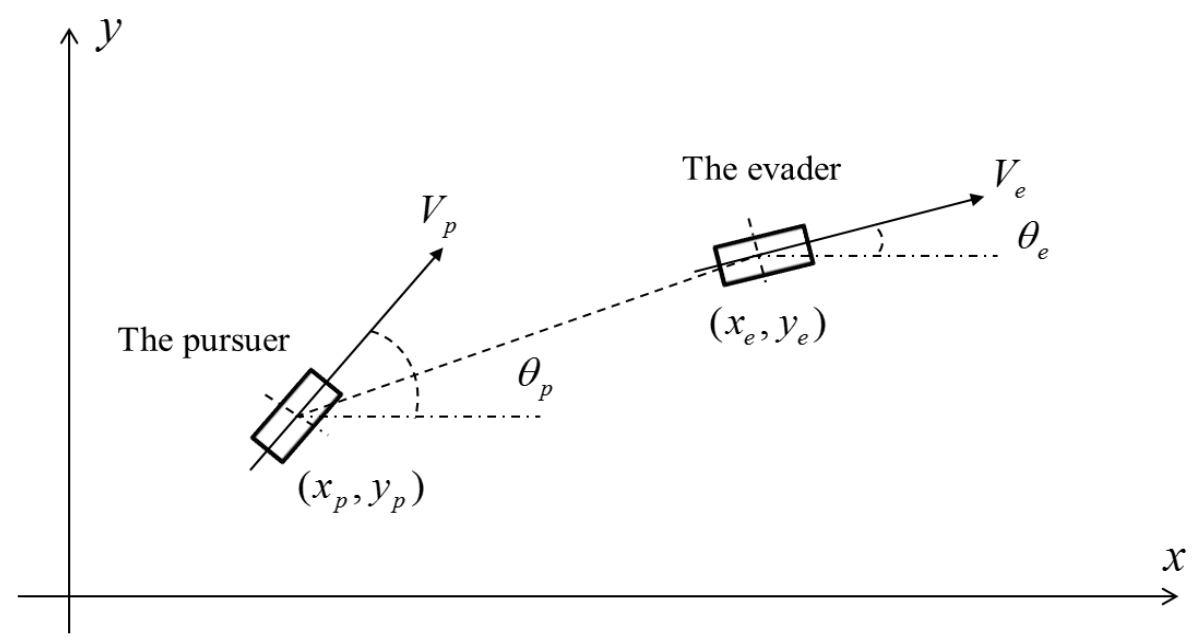

Figure 4.2: Pursuit-evasion model.

$$
\begin{gathered}
\dot{x}_{\kappa}=V_{\kappa} \cos \left(\theta_{\kappa}\right), \\
\dot{y}_{\kappa}=V_{\kappa} \sin \left(\theta_{\kappa}\right), \\
\dot{\theta}_{\kappa}=\frac{V_{\kappa}}{L_{\kappa}} \tan \left(u_{\kappa}\right),
\end{gathered}
$$

where $\kappa$ represents both the pursuer " $p$ " and the evader " $e$ ", $V_{\kappa}$ represents robot $\kappa$ 's speed, $\theta_{\kappa}$ is the orientation of robot $\kappa,\left(x_{\kappa}, y_{\kappa}\right)$ is the position of robot $\kappa, L_{\kappa}$ represents the wheelbase of robot $\kappa$, and $u_{\kappa}$ represents robot $\kappa$ 's steering angle, where $u_{\kappa} \in\left[-u_{\kappa_{\max }}, u_{\kappa_{\max }}\right]$.

In this work, we assume that the pursuer is faster than the evader by making $V_{p}>V_{e}$. It is also assumed that the evader is more maneuverable than the pursuer by making $u_{e_{\max }}>u_{p_{\max }}$. A simple classical control strategy that can be used to define the control strategies of the pursuer and the evader, in a pursuit-evasion game, can be given as follows, 


$$
u_{\kappa}=\left\{\begin{aligned}
-u_{\kappa_{\max }} & : \delta_{\kappa}<-u_{\kappa_{\max }}, \\
\delta_{\kappa} & :-u_{\kappa_{\max }} \leq \delta_{\kappa} \leq u_{\kappa_{\max }}, \\
u_{\kappa_{\max }} & : \delta_{\kappa}>u_{\kappa_{\max }},
\end{aligned}\right.
$$

and,

$$
\delta_{\kappa}=\tan ^{-1}\left(\frac{y_{e}-y_{p}}{x_{e}-x_{p}}\right)-\theta_{\kappa}
$$

where $\delta_{\kappa}$ represents the angle difference between the direction of robot $\kappa$ and the pursuer's line-of-sight (LoS) to the evader.

To capture the evader in a pursuit-evasion game when the pursuer uses the simple control strategy described by Eq (4.28) and Eq (4.29), the angle difference $\delta_{p}$ has to be driven to zero by the pursuer. Thus, the control strategy of the pursuer in this case is to drive this angle difference to zero. On the other hand, the control strategy of the evader is to escape from the pursuer and keep the distance between the evader and the pursuer as large as possible. The evader can do so by following the intelligent control strategy described by the following two rules $[100,121,135,136]$ :

1. If the distance between the evader and the pursuer is greater than a specific distance $\bar{d}$, the control strategy of the evader is defined as follows,

$$
u_{e}=\tan ^{-1}\left(\frac{y_{e}-y_{p}}{x_{e}-x_{p}}\right)-\theta_{e}
$$

2. If the distance between the evader and the pursuer is less than or equal to the distance $\bar{d}$, the evader exploits its higher maneuverability, and the control strategy for the evader in this case is given as follows, 


$$
u_{e}=\left(\theta_{p}+\pi\right)-\theta_{e}
$$

The distance $\bar{d}$ is defined as follows,

$$
\bar{d}=\frac{L_{p}}{\tan \left(u_{p_{\max }}\right)} .
$$

The pursuer succeeds to capture the evader if the distance between them is less than the capture radius $d_{c}$. The distance between the pursuer and the evader is defined by $d$ and is given as follows,

$$
d=\sqrt{\left(x_{e}-x_{p}\right)^{2}+\left(y_{e}-y_{p}\right)^{2}} .
$$

\subsection{The Proposed Algorithm}

In this section, we propose a new fuzzy reinforcement learning algorithm for differential games that have continuous state and action spaces. The proposed algorithm uses FISs as FASs; an actor (fuzzy logic controller, FLC), and a critic. The critic is used to estimate the value functions $V_{t}\left(\mathbf{s}_{t}\right)$ and $V_{t}\left(\mathbf{s}_{t+1}\right)$ of the learning agent at two different states, $\mathbf{s}_{t}$ and $\mathbf{s}_{t+1}$ respectively. The values of $V_{t}\left(\mathbf{s}_{t}\right)$ and $V_{t}\left(\mathbf{s}_{t+1}\right)$ will depend on the input and output parameters of the critic. Unlike the algorithms proposed in $[48,99,100,103,126,127]$ which use the direct algorithms to tune the parameters of their FASs, the proposed algorithm uses the residual gradient value iteration algorithm described in [113] to tune the input and output parameters of its FASs. It has been shown in $[113,129-132]$ that the direct algorithms may not converge to an answer in some cases, while the residual gradient algorithms are always guaranteed to converge. The proposed algorithm is called the residual gradient fuzzy actor critic learning (RGFACL) algorithm. The structure of the proposed RGFACL algorithm 
is shown in Fig. (4.3). The input parameters of the critic are the parameters of the MFs of its inputs, $\sigma_{\jmath}$ and $m_{\jmath}($ where $\jmath=1, \ldots, H)$. The output parameters of the critic are the consequent parameters of its rules, $k_{l}$ (where $l=1, \ldots, L$ ). To simplify notations, we refer to the input and output parameters of the critic as $\psi^{C}$. Similarly, the input parameters of the actor are the parameters of the MFs of its input $\sigma_{\jmath}$ and $m_{\jmath}$, and the output parameters of the actor are the consequent parameters of its rules $k_{l}$. To simplify notations, we refer to the input and the output parameters of the actor as $\psi^{A}$. The temporal difference residual error, $\Delta_{t}$, is defined as follows,

$$
\Delta_{t}=r_{t}+\zeta V_{t}\left(\mathbf{s}_{t+1}\right)-V_{t}\left(\mathbf{s}_{t}\right)
$$

\subsubsection{Adaptation Rules for the Critic}

In this subsection, we derive the adaptation rules that the proposed algorithm uses to tune the input and the output parameters of the critic. The mean square error, $E$, of the temporal difference residual error, $\Delta_{t}$, is defined as follows,

$$
E=\frac{1}{2} \Delta_{t}^{2}
$$

The input and output parameters of the critic are updated based on the residual gradient method described in [113] as follows,

$$
\psi_{t+1}^{C}=\psi_{t}^{C}-\rho^{C} \frac{\partial E}{\partial \psi_{t}^{C}},
$$

where $\psi_{t}^{C}$ represents the input and output parameters of the critic at time $t$, and $\rho^{C}$ is a learning rate for the parameters of the critic.

The QLFIS algorithm proposed in [100] uses the so-called direct algorithms described 
in [113] to define the term $\frac{\partial E}{\partial \psi_{t}^{C}}$. On the other hand, the proposed algorithm defines the term $\frac{\partial E}{\partial \psi_{t}^{C}}$ based on the residual gradient value iteration algorithm which is also described in [113] as follows,

$$
\frac{\partial E}{\partial \psi_{t}^{C}}=\Delta_{t}\left[\zeta \frac{\partial V_{t}\left(\mathbf{s}_{t+1}\right)}{\partial \psi_{t}^{C}}-\frac{\partial V_{t}\left(\mathbf{s}_{t}\right)}{\partial \psi_{t}^{C}}\right] .
$$

The proposed algorithm treats the value function $V_{t}\left(\mathbf{s}_{t+1}\right)$ in the temporal difference residual error $\Delta_{t}$ as a function of the input and the output parameters of its FAS (the critic), $\psi_{t}^{C}$. Unlike the QLFIS algorithm, the proposed algorithm will not assign a value of zero to the derivative $\frac{\partial V_{t}\left(\mathbf{s}_{t+1}\right)}{\partial \psi_{t}^{C}}$ during the tuning of the input and the output parameters of the critic. This is because the value function $V_{t}\left(\mathbf{s}_{t+1}\right)$ is a function of the input and the output parameters of the critic $\psi_{t}^{C}$, and its derivative $\frac{\partial V_{t}\left(\mathbf{s}_{t+1}\right)}{\partial \psi_{t}^{C}}$ should not be assigned to zero all the time.

From Eq. (4.37), Eq. (4.36) can be rewritten as follows,

$$
\psi_{t+1}^{C}=\psi_{t}^{C}-\rho^{C}\left[r_{t}+\zeta V_{t}\left(\mathbf{s}_{t+1}\right)-V_{t}\left(\mathbf{s}_{t}\right)\right] \cdot\left[\zeta \frac{\partial}{\partial \psi_{t}^{C}} V_{t}\left(\mathbf{s}_{t+1}\right)-\frac{\partial}{\partial \psi_{t}^{C}} V_{t}\left(\mathbf{s}_{t}\right)\right]
$$

The derivatives of the state value functions, $V_{t}\left(\mathbf{s}_{t}\right)$ and $V_{t}\left(\mathbf{s}_{t+1}\right)$, with respect to the output parameters of the critic, $k_{l}$, is calculated from Eq. (4.14) as follows,

$$
\frac{\partial V_{t}\left(\mathbf{s}_{t}\right)}{\partial k_{l}}=\Phi_{l}\left(\mathbf{s}_{t}\right)
$$

where $l=1, \ldots, L$.

Similarly, 


$$
\frac{\partial V_{t}\left(\mathbf{s}_{t+1}\right)}{\partial k_{l}}=\Phi_{l}\left(\mathbf{s}_{t+1}\right)
$$

where $\Phi_{l}\left(\mathbf{s}_{t}\right)$ and $\Phi_{l}\left(\mathbf{s}_{t+1}\right)$ are calculated by using Eq. (4.15) at the states $\mathbf{s}_{t}$ and $\mathbf{s}_{t+1}$, respectively.

We use the chain rule to calculate the derivatives $\frac{\partial V_{t}\left(\mathbf{s}_{t}\right)}{\partial \sigma_{\jmath}}$ and $\frac{\partial V_{t}\left(\mathbf{s}_{t+1}\right)}{\partial \sigma_{\jmath}}$. We start with the derivative $\frac{\partial V_{t}\left(\mathbf{s}_{t}\right)}{\partial \sigma_{\jmath}}$ which is calculated as follows,

$$
\frac{\partial V_{t}\left(\mathbf{s}_{t}\right)}{\partial \sigma_{\jmath}}=\frac{\partial V_{t}\left(\mathbf{s}_{t}\right)}{\partial \omega_{l}\left(\mathbf{s}_{t}\right)} \cdot \frac{\partial \omega_{l}\left(\mathbf{s}_{t}\right)}{\partial \sigma_{\jmath}}
$$

where $\jmath=1, \ldots, H$.

The term $\frac{\partial V_{t}\left(\mathbf{s}_{t}\right)}{\partial \omega_{l}\left(\mathbf{s}_{t}\right)}$ is calculated as follows,

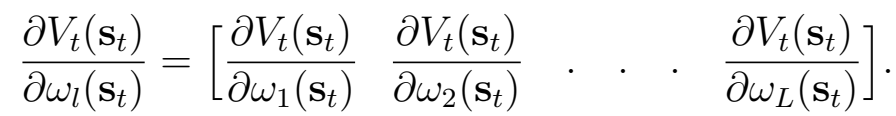

We first calculate the term $\frac{\partial V_{t}\left(\mathbf{s}_{t}\right)}{\partial \omega_{1}\left(\mathbf{s}_{t}\right)}$ as follows,

$$
\frac{\partial V_{t}\left(\mathbf{s}_{t}\right)}{\partial \omega_{1}\left(\mathbf{s}_{t}\right)}=\frac{\partial}{\partial \omega_{1}\left(\mathbf{s}_{t}\right)}\left[\frac{\sum_{l} \omega_{l}\left(\mathbf{s}_{t}\right) k_{l}}{\sum_{l} \omega_{l}\left(\mathbf{s}_{t}\right)}\right]=\frac{k_{1}-V_{t}\left(\mathbf{s}_{t}\right)}{\sum_{l} \omega_{l}\left(\mathbf{s}_{t}\right)}
$$

Similarly, we can calculate the terms $\frac{\partial V_{t}\left(\mathbf{s}_{t}\right)}{\partial \omega_{2}\left(\mathbf{s}_{t}\right)}, \ldots$, and $\frac{\partial V_{t}\left(\mathbf{s}_{t}\right)}{\partial \omega_{L}\left(\mathbf{s}_{t}\right)}$. Thus, Eq. (4.42) can be rewritten as follows,

$$
\frac{\partial V_{t}\left(\mathbf{s}_{t}\right)}{\partial \omega_{l}\left(\mathbf{s}_{t}\right)}=\left[\frac{k_{1}-V_{t}\left(\mathbf{s}_{t}\right)}{\sum_{l} \omega_{l}\left(\mathbf{s}_{t}\right)} \frac{k_{2}-V_{t}\left(\mathbf{s}_{t}\right)}{\sum_{l} \omega_{l}\left(\mathbf{s}_{t}\right)} \quad . \quad . \quad \frac{k_{L}-V_{t}\left(\mathbf{s}_{t}\right)}{\sum_{l} \omega_{l}\left(\mathbf{s}_{t}\right)}\right] .
$$

On the other hand, the term $\frac{\partial \omega_{l}\left(\mathbf{s}_{t}\right)}{\partial \sigma_{\jmath}}$ in Eq. (4.41) is calculated as follows,

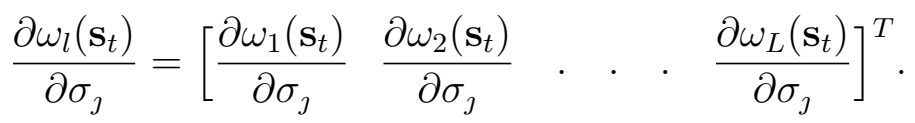


The derivative $\frac{\partial \omega_{1}\left(\mathbf{s}_{t}\right)}{\partial \sigma_{\jmath}}$ can be calculated based on the definition of $\omega_{1}\left(\mathbf{s}_{t}\right)$ given in Eq. (4.16) as follows,

$$
\frac{\partial \omega_{1}\left(\mathbf{s}_{t}\right)}{\partial \sigma_{\jmath}}=\frac{\partial}{\partial \sigma_{\jmath}}\left[\prod_{\imath=1}^{n} \mu^{A_{\imath}^{1}}\left(s_{\imath}\right)\right]=\frac{\partial}{\partial \sigma_{\jmath}}\left[\mu^{A_{1}^{1}}\left(s_{1}\right) \times \mu^{A_{2}^{1}}\left(s_{2}\right) \times \quad . \quad . \quad \times \mu^{A_{n}^{1}}\left(s_{n}\right)\right] .
$$

We then substitute Eq. (4.12) into Eq. (4.46) as follows,

$$
\begin{aligned}
\frac{\partial \omega_{1}\left(\mathbf{s}_{t}\right)}{\partial \sigma_{\jmath}}=\frac{\partial}{\partial \sigma_{\jmath}}\left[\exp \left(-\left(\frac{s_{1}-m_{1}}{\sigma_{1}}\right)^{2}\right) \times \exp (\right. & \left.-\left(\frac{s_{2}-m_{h+1}}{\sigma_{h+1}}\right)^{2}\right) \times \ldots . \\
& \left.\times \exp \left(-\left(\frac{s_{n}-m_{(n-1) h+1}}{\sigma_{(n-1) h+1}}\right)^{2}\right)\right] .
\end{aligned}
$$

Thus, the derivatives $\frac{\partial \omega_{1}\left(\mathbf{s}_{t}\right)}{\partial \sigma_{1}}, \frac{\partial \omega_{1}\left(\mathbf{s}_{t}\right)}{\partial \sigma_{2}}, \ldots$, and $\frac{\partial \omega_{1}\left(\mathbf{s}_{t}\right)}{\partial \sigma_{H}}$ are calculated as follows, 


$$
\begin{aligned}
& \frac{\partial \omega_{1}\left(\mathbf{s}_{t}\right)}{\partial \sigma_{1}}=\frac{2\left(s_{1}-m_{1}\right)^{2}}{\sigma_{1}^{3}} \omega_{1}\left(\mathbf{s}_{t}\right), \\
& \frac{\partial \omega_{1}\left(\mathbf{s}_{t}\right)}{\partial \sigma_{2}}=0,
\end{aligned}
$$

$$
\begin{aligned}
& \frac{\partial \omega_{1}\left(\mathbf{s}_{t}\right)}{\partial \sigma_{h+1}}=\frac{2\left(s_{2}-m_{h+1}\right)^{2}}{\sigma_{h+1}^{3}} \omega_{1}\left(\mathbf{s}_{t}\right), \\
& \frac{\partial \omega_{1}\left(\mathbf{s}_{t}\right)}{\partial \sigma_{h+2}}=0
\end{aligned}
$$

$$
\begin{aligned}
\frac{\partial \omega_{1}\left(\mathbf{s}_{t}\right)}{\partial \sigma_{(n-1) h+1}} & =\frac{2\left(s_{n}-m_{(n-1) h+1}\right)^{2}}{\sigma_{(n-1) h+1}^{3}} \omega_{1}\left(\mathbf{s}_{t}\right), \\
\frac{\partial \omega_{1}\left(\mathbf{s}_{t}\right)}{\partial \sigma_{(n-1) h+2}} & =0,
\end{aligned}
$$

$$
\frac{\partial \omega_{1}\left(\mathbf{s}_{t}\right)}{\partial \sigma_{H}}=0
$$

Thus, from Eq. (4.48), the derivative $\frac{\partial \omega_{1}\left(\mathbf{s}_{t}\right)}{\partial \sigma_{\jmath}},(\jmath=1, \ldots, H)$, can be rewritten as follows, 


$$
\frac{\partial \omega_{1}\left(\mathbf{s}_{t}\right)}{\partial \sigma_{\jmath}}=\left\{\begin{array}{cl}
\frac{2\left(s_{\imath}-m_{\jmath}\right)^{2}}{\sigma_{\jmath}^{3}} \omega_{1}\left(\mathbf{s}_{t}\right) & \text { if } \quad\left(\sigma_{\jmath}, m_{\jmath}\right) \in \Omega\left(\omega_{1}\right), \\
0 & \text { if } \quad\left(\sigma_{\jmath}, m_{\jmath}\right) \notin \Omega\left(\omega_{1}\right),
\end{array}\right.
$$

where the term $s_{\imath}$ is the $\imath$ th input state variable of the state vector $\mathbf{s}_{t}$ and is defined as follows,

$$
s_{\imath}=\left\{\begin{array}{ccc}
s_{1} & \text { if } \quad\left(\sigma_{\jmath}, m_{\jmath}\right) \in \Omega\left(s_{1}\right), \\
s_{2} & \text { if } \quad\left(\sigma_{\jmath}, m_{\jmath}\right) \in \Omega\left(s_{2}\right), \\
& \cdot & \\
\cdot & \\
& \cdot & \\
s_{n} & \text { if } \quad\left(\sigma_{\jmath}, m_{\jmath}\right) \in \Omega\left(s_{n}\right) .
\end{array}\right.
$$

We can rewrite Eq. (4.49) as follows,

$$
\frac{\partial \omega_{1}\left(\mathbf{s}_{t}\right)}{\partial \sigma_{\jmath}}=\xi_{\jmath, 1} \frac{2\left(s_{\imath}-m_{\jmath}\right)^{2}}{\sigma_{\jmath}^{3}} \omega_{1}\left(\mathbf{s}_{t}\right),
$$

where,

$$
\xi_{\jmath, 1}=\left\{\begin{array}{ccc}
1 & \text { if } & \left(\sigma_{\jmath}, m_{\jmath}\right) \in \Omega\left(\omega_{1}\right), \\
0 & \text { if } & \left(\sigma_{\jmath}, m_{\jmath}\right) \notin \Omega\left(\omega_{1}\right) .
\end{array}\right.
$$

Similarly, we can calculate the derivatives $\frac{\partial \omega_{2}\left(\mathbf{s}_{t}\right)}{\partial \sigma_{\jmath}}$ as follows, 


$$
\begin{array}{r}
\frac{\partial \omega_{2}\left(\mathbf{s}_{t}\right)}{\partial \sigma_{\jmath}}=\frac{\partial}{\partial \sigma_{\jmath}}\left[\prod_{\imath=1}^{n} \mu^{A_{\imath}^{2}}\left(s_{\imath}\right)\right]=\frac{\partial}{\partial \sigma_{\jmath}}\left[\mu^{A_{1}^{2}}\left(s_{1}\right) \times \mu^{A_{2}^{2}}\left(s_{2}\right) \times \quad . \quad . \quad \times \mu^{A_{n}^{2}}\left(s_{n}\right)\right] \\
\begin{aligned}
\frac{\partial \omega_{2}\left(\mathbf{s}_{t}\right)}{\partial \sigma_{\jmath}}=\frac{\partial}{\partial \sigma_{\jmath}}\left[\exp \left(-\left(\frac{s_{1}-m_{1}}{\sigma_{1}}\right)^{2}\right) \times \exp \left(-\left(\frac{s_{2}-m_{h+1}}{\sigma_{h+1}}\right)^{2}\right) \times\right. & \cdot \\
& \left.\times \exp \left(-\left(\frac{s_{n}-m_{(n-1) h+2}}{\sigma_{(n-1) h+2}}\right)^{2}\right)\right]
\end{aligned}
\end{array}
$$

Thus, the derivatives $\frac{\partial \omega_{2}\left(\mathbf{s}_{t}\right)}{\partial \sigma_{1}}, \ldots$, and $\frac{\partial \omega_{2}\left(\mathbf{s}_{t}\right)}{\partial \sigma_{H}}$ are calculated as follows, 


$$
\begin{aligned}
\frac{\partial \omega_{2}\left(\mathbf{s}_{t}\right)}{\partial \sigma_{1}} & =\frac{2\left(s_{1}-m_{1}\right)^{2}}{\sigma_{1}^{3}} \omega_{2}\left(\mathbf{s}_{t}\right), \\
\frac{\partial \omega_{2}\left(\mathbf{s}_{t}\right)}{\partial \sigma_{2}} & =0
\end{aligned}
$$

$$
\begin{aligned}
\frac{\partial \omega_{2}\left(\mathbf{s}_{t}\right)}{\partial \sigma_{h+1}} & =\frac{2\left(s_{2}-m_{h+1}\right)^{2}}{\sigma_{h+1}^{3}} \omega_{2}\left(\mathbf{s}_{t}\right), \\
\frac{\partial \omega_{2}\left(\mathbf{s}_{t}\right)}{\partial \sigma_{h+2}} & =0,
\end{aligned}
$$

$$
\begin{aligned}
\frac{\partial \omega_{2}\left(\mathbf{s}_{t}\right)}{\partial \sigma_{(n-1) h+1}} & =0, \\
\frac{\partial \omega_{2}\left(\mathbf{s}_{t}\right)}{\partial \sigma_{(n-1) h+2}} & =\frac{2\left(s_{n}-m_{(n-1) h+2}\right)^{2}}{\sigma_{(n-1) h+2}^{3}} \omega_{2}\left(\mathbf{s}_{t}\right), \\
\frac{\partial \omega_{2}\left(\mathbf{s}_{t}\right)}{\partial \sigma_{(n-1) h+3}} & =0,
\end{aligned}
$$

$$
\frac{\partial \omega_{2}\left(\mathbf{s}_{t}\right)}{\partial \sigma_{H}}=0 .
$$

Thus, from Eq. (4.55), the derivatives $\frac{\partial \omega_{2}\left(\mathrm{~s}_{t}\right)}{\partial \sigma_{\jmath}},(\jmath=1, \ldots, H)$, can be rewritten as 
follows,

$$
\frac{\partial \omega_{2}\left(\mathbf{s}_{t}\right)}{\partial \sigma_{\jmath}}=\left\{\begin{array}{ccc}
\frac{2\left(s_{\imath}-m_{\jmath}\right)^{2}}{\sigma_{\jmath}^{3}} \omega_{2}\left(\mathbf{s}_{t}\right) & \text { if } & \left(\sigma_{\jmath}, m_{\jmath}\right) \in \Omega\left(\omega_{2}\right), \\
0 & \text { if } \quad\left(\sigma_{\jmath}, m_{\jmath}\right) \notin \Omega\left(\omega_{2}\right) .
\end{array}\right.
$$

We can rewrite Eq. (4.56) as follows,

$$
\frac{\partial \omega_{2}\left(\mathbf{s}_{t}\right)}{\partial \sigma_{\jmath}}=\xi_{\jmath, 2} \frac{2\left(s_{\imath}-m_{\jmath}\right)^{2}}{\sigma_{\jmath}^{3}} \omega_{2}\left(\mathbf{s}_{t}\right),
$$

where,

$$
\xi_{\jmath, 2}=\left\{\begin{array}{ccc}
1 & \text { if } & \left(\sigma_{\jmath}, m_{\jmath}\right) \in \Omega\left(\omega_{2}\right), \\
0 & \text { if } \quad\left(\sigma_{\jmath}, m_{\jmath}\right) \notin \Omega\left(\omega_{2}\right) .
\end{array}\right.
$$

Similarly, we can calculate the derivatives $\frac{\partial \omega_{3}\left(\mathbf{s}_{t}\right)}{\partial \sigma_{\jmath}}, \frac{\partial \omega_{4}\left(\mathbf{s}_{t}\right)}{\partial \sigma_{\jmath}}, \ldots$, and $\frac{\partial \omega_{L}\left(\mathbf{s}_{t}\right)}{\partial \sigma_{\jmath}}$. Thus, Eq. (4.45) can be rewritten as follows,

$$
\begin{array}{r}
\frac{\partial \omega_{l}\left(\mathbf{s}_{t}\right)}{\partial \sigma_{\jmath}}=\left[\begin{array}{ll}
\xi_{\jmath, 1} \frac{2\left(s_{\imath}-m_{\jmath}\right)^{2}}{\sigma_{\jmath}^{3}} \omega_{1}\left(\mathbf{s}_{t}\right) & \xi_{\jmath, 2} \frac{2\left(s_{\imath}-m_{\jmath}\right)^{2}}{\sigma_{\jmath}^{3}} \omega_{2}\left(\mathbf{s}_{t}\right), \quad . \\
\xi_{\jmath, L} \frac{2\left(s_{\imath}-m_{\jmath}\right)^{2}}{\sigma_{\jmath}^{3}} \omega_{L}\left(\mathbf{s}_{t}\right)
\end{array}\right]^{T},
\end{array}
$$

where,

$$
\xi_{\jmath, l}=\left\{\begin{array}{cc}
1 & \text { if } \quad\left(\sigma_{\jmath}, m_{\jmath}\right) \in \Omega\left(\omega_{l}\right) \\
0 & \text { if } \quad\left(\sigma_{\jmath}, m_{\jmath}\right) \notin \Omega\left(\omega_{l}\right) .
\end{array}\right.
$$

Hence, from Eq. (4.44) and Eq. (4.59), the derivative $\frac{\partial V_{t}\left(\mathbf{s}_{t}\right)}{\partial \sigma_{\jmath}},(\jmath=1, \ldots, H)$, in Eq. (4.41) is then calculated as follows, 


$$
\frac{\partial V_{t}\left(\mathbf{s}_{t}\right)}{\partial \sigma_{\jmath}}=\frac{2\left(s_{\imath}-m_{\jmath}\right)^{2}}{\sigma_{\jmath}^{3}} \times \sum_{l=1}^{L} \xi_{\jmath, l} \frac{k_{l}-V_{t}\left(\mathbf{s}_{t}\right)}{\sum_{l} \omega_{l}\left(\mathbf{s}_{t}\right)} \omega_{l}\left(\mathbf{s}_{t}\right) .
$$

Similarly, we calculate the derivative $\frac{\partial V_{t}\left(\mathbf{s}_{t+1}\right)}{\partial \sigma_{\jmath}}$ as follows,

$$
\frac{\partial V_{t}\left(\mathbf{s}_{t+1}\right)}{\partial \sigma_{\jmath}}=\frac{2\left(s_{\imath}^{\prime}-m_{\jmath}\right)^{2}}{\sigma_{\jmath}^{3}} \times \sum_{l=1}^{L} \xi_{\jmath}, l \frac{k_{l}-V_{t}\left(\mathbf{s}_{t+1}\right)}{\sum_{l} \omega_{l}\left(\mathbf{s}_{t+1}\right)} \omega_{l}\left(\mathbf{s}_{t+1}\right),
$$

where,

$$
s_{\imath}^{\prime}=\left\{\begin{array}{ccc}
s_{1}^{\prime} & \text { if } \quad\left(\sigma_{\jmath}, m_{\jmath}\right) \in \Omega\left(s_{1}\right), \\
s_{2}^{\prime} & \text { if } \quad\left(\sigma_{\jmath}, m_{\jmath}\right) \in \Omega\left(s_{2}\right), \\
& \cdot & \\
& \cdot & \\
& \cdot & \\
s_{n}^{\prime} & \text { if } \quad\left(\sigma_{\jmath}, m_{\jmath}\right) \in \Omega\left(s_{n}\right),
\end{array}\right.
$$

where $s_{\imath}^{\prime}$ is the $\imath$ th input state variable of the state vector $\mathbf{s}_{t+1}$.

We also use the chain rule to calculate the derivatives $\frac{\partial V_{t}\left(\mathbf{s}_{t}\right)}{\partial m_{\jmath}}$ and $\frac{\partial V_{t}\left(\mathbf{s}_{t+1}\right)}{\partial m_{\jmath}}$. We start with the derivative $\frac{\partial V_{t}\left(\mathbf{s}_{t}\right)}{\partial m_{\jmath}}$ which is calculated as follows,

$$
\frac{\partial V_{t}\left(\mathbf{s}_{t}\right)}{\partial m_{\jmath}}=\frac{\partial V_{t}\left(\mathbf{s}_{t}\right)}{\partial \omega_{l}\left(\mathbf{s}_{t}\right)} \cdot \frac{\partial \omega_{l}\left(\mathbf{s}_{t}\right)}{\partial m_{\jmath}}
$$

The term $\frac{\partial V_{t}\left(\mathbf{s}_{t}\right)}{\partial \omega_{l}\left(\mathbf{s}_{t}\right)}$ is calculated as in Eq. (4.44) and the term $\frac{\partial \omega_{l}\left(\mathbf{s}_{t}\right)}{\partial m_{\jmath}}$ is calculated as follows,

$$
\frac{\partial \omega_{l}\left(\mathbf{s}_{t}\right)}{\partial m_{\jmath}}=\left[\frac{\partial \omega_{1}\left(\mathbf{s}_{t}\right)}{\partial m_{\jmath}} \frac{\partial \omega_{2}\left(\mathbf{s}_{t}\right)}{\partial m_{\jmath}} \quad \cdot . \frac{\partial \omega_{L}\left(\mathbf{s}_{t}\right)}{\partial m_{\jmath}}\right]^{T} .
$$


We first calculate the term $\frac{\partial \omega_{1}\left(\mathbf{s}_{t}\right)}{\partial m_{\jmath}}$ by using Eq. (4.16) as follows,

$$
\frac{\partial \omega_{1}\left(\mathbf{s}_{t}\right)}{\partial m_{\jmath}}=\frac{\partial}{\partial m_{\jmath}}\left[\prod_{\imath=1}^{n} \mu^{A_{\imath}^{1}}\left(s_{\imath}\right)\right]=\frac{\partial}{\partial m_{\jmath}}\left[\mu^{A_{1}^{1}}\left(s_{1}\right) \times \mu^{A_{2}^{1}}\left(s_{2}\right) \times \quad . \quad . \quad \times \mu^{A_{n}^{1}}\left(s_{n}\right)\right] .
$$

We then substitute Eq. (4.12) into Eq. (4.66) as follows,

$$
\begin{aligned}
\frac{\partial \omega_{1}\left(\mathbf{s}_{t}\right)}{\partial m_{\text {J }}}=\frac{\partial}{\partial m_{\jmath}}\left[\exp \left(-\left(\frac{s_{1}-m_{1}}{\sigma_{1}}\right)^{2}\right) \times \exp (\right. & \left.-\left(\frac{s_{2}-m_{h+1}}{\sigma_{h+1}}\right)^{2}\right) \times \quad . \quad . \\
& \left.\times \exp \left(-\left(\frac{s_{n}-m_{(n-1) h+1}}{\sigma_{(n-1) h+1}}\right)^{2}\right)\right]
\end{aligned}
$$

Thus, the derivatives $\frac{\partial \omega_{1}\left(\mathbf{s}_{t}\right)}{\partial m_{1}}, \frac{\partial \omega_{1}\left(\mathbf{s}_{t}\right)}{\partial m_{2}}, \ldots$, and $\frac{\partial \omega_{1}\left(\mathbf{s}_{t}\right)}{\partial m_{H}}$ are calculated as follows, 


$$
\begin{aligned}
& \frac{\partial \omega_{1}\left(\mathbf{s}_{t}\right)}{\partial m_{1}}=\frac{2\left(s_{1}-m_{1}\right)}{\sigma_{1}^{2}} \omega_{1}\left(\mathbf{s}_{t}\right), \\
& \frac{\partial \omega_{1}\left(\mathbf{s}_{t}\right)}{\partial m_{2}}=0
\end{aligned}
$$

$$
\begin{aligned}
\frac{\partial \omega_{1}\left(\mathbf{s}_{t}\right)}{\partial m_{h+1}} & =\frac{2\left(s_{2}-m_{h+1}\right)}{\sigma_{h+1}^{2}} \omega_{1}\left(\mathbf{s}_{t}\right), \\
\frac{\partial \omega_{1}\left(\mathbf{s}_{t}\right)}{\partial m_{h+2}} & =0,
\end{aligned}
$$

$$
\begin{aligned}
\frac{\partial \omega_{1}\left(\mathbf{s}_{t}\right)}{\partial m_{(n-1) h+1}} & =\frac{2\left(s_{n}-m_{(n-1) h+1}\right)}{\sigma_{(n-1) h+1}^{2}} \omega_{1}\left(\mathbf{s}_{t}\right), \\
\frac{\partial \omega_{1}\left(\mathbf{s}_{t}\right)}{\partial m_{(n-1) h+2}} & =0,
\end{aligned}
$$

$$
\frac{\partial \omega_{1}\left(\mathbf{s}_{t}\right)}{\partial m_{H}}=0
$$

Thus, from Eq. (4.68), the derivative $\frac{\partial \omega_{1}\left(\mathbf{s}_{t}\right)}{\partial m_{\jmath}},(\jmath=1, \ldots, H)$, can be rewritten as follows, 


$$
\frac{\partial \omega_{1}\left(\mathbf{s}_{t}\right)}{\partial m_{\jmath}}=\left\{\begin{array}{ccc}
\frac{2\left(s_{\imath}-m_{\jmath}\right)}{\sigma_{\jmath}^{2}} \omega_{1}\left(\mathbf{s}_{t}\right) & \text { if } \quad\left(\sigma_{\jmath}, m_{\jmath}\right) \in \Omega\left(\omega_{1}\right), \\
0 & \text { if } \quad\left(\sigma_{\jmath}, m_{\jmath}\right) \notin \Omega\left(\omega_{1}\right) .
\end{array}\right.
$$

We can rewrite Eq. (4.69) as follows,

$$
\frac{\partial \omega_{1}\left(\mathbf{s}_{t}\right)}{\partial m_{\jmath}}=\xi_{\jmath, 1} \frac{2\left(s_{\imath}-m_{\jmath}\right)}{\sigma_{\jmath}^{2}} \omega_{1}\left(\mathbf{s}_{t}\right),
$$

where $s_{\imath}$ is defined as in Eq. (4.50), and $\xi_{\jmath, 1}$ is defined as in Eq. (4.52).

Similarly, we can calculate the term $\frac{\partial \omega_{2}\left(\mathbf{s}_{t}\right)}{\partial m_{\jmath}}$ as follows,

$$
\begin{aligned}
& \begin{array}{l}
\frac{\partial \omega_{2}\left(\mathbf{s}_{t}\right)}{\partial m_{\jmath}}=\frac{\partial}{\partial m_{\jmath}}\left[\prod_{\imath=1}^{n} \mu^{A_{\imath}^{2}}\left(s_{\imath}\right)\right]=\frac{\partial}{\partial m_{\jmath}}\left[\mu^{A_{1}^{2}}\left(s_{1}\right) \times \mu^{A_{2}^{2}}\left(s_{2}\right) \times . . \quad \times \mu^{A_{n}^{2}}\right. \\
\frac{\partial \omega_{2}\left(\mathbf{s}_{t}\right)}{\partial m_{\jmath}}=\frac{\partial}{\partial m_{\jmath}}\left[\exp \left(-\left(\frac{s_{1}-m_{1}}{\sigma_{1}}\right)^{2}\right) \times \exp \left(-\left(\frac{s_{2}-m_{h+1}}{\sigma_{h+1}}\right)^{2}\right) \times \quad . \quad .\right.
\end{array} \\
& \left.\times \exp \left(-\left(\frac{s_{n}-m_{(n-1) h+2}}{\sigma_{(n-1) h+2}}\right)^{2}\right)\right] .
\end{aligned}
$$

Thus, the derivatives $\frac{\partial \omega_{2}\left(\mathbf{s}_{t}\right)}{\partial m_{1}}, \frac{\partial \omega_{2}\left(\mathbf{s}_{t}\right)}{\partial m_{2}}, \ldots$, and $\frac{\partial \omega_{2}\left(\mathbf{s}_{t}\right)}{\partial m_{H}}$ are calculated as follows, 


$$
\begin{aligned}
& \frac{\partial \omega_{2}\left(\mathbf{s}_{t}\right)}{\partial m_{1}}=\frac{2\left(s_{1}-m_{1}\right)}{\sigma_{1}^{2}} \omega_{2}\left(\mathbf{s}_{t}\right) \\
& \frac{\partial \omega_{2}\left(\mathbf{s}_{t}\right)}{\partial m_{2}}=0
\end{aligned}
$$

$$
\begin{aligned}
& \frac{\partial \omega_{2}\left(\mathbf{s}_{t}\right)}{\partial m_{h+1}}=\frac{2\left(s_{2}-m_{h+1}\right)}{\sigma_{h+1}^{2}} \omega_{2}\left(\mathbf{s}_{t}\right) \\
& \frac{\partial \omega_{2}\left(\mathbf{s}_{t}\right)}{\partial m_{h+2}}=0
\end{aligned}
$$

$$
\begin{aligned}
\frac{\partial \omega_{2}\left(\mathbf{s}_{t}\right)}{\partial m_{(n-1) h+1}} & =0 \\
\frac{\partial \omega_{2}\left(\mathbf{s}_{t}\right)}{\partial m_{(n-1) h+2}} & =\frac{2\left(s_{n}-m_{(n-1) h+2}\right)}{\sigma_{(n-1) h+2}^{2}} \omega_{2}\left(\mathbf{s}_{t}\right) \\
\frac{\partial \omega_{2}\left(\mathbf{s}_{t}\right)}{\partial m_{(n-1) h+3}} & =0
\end{aligned}
$$

$$
\frac{\partial \omega_{2}\left(\mathbf{s}_{t}\right)}{\partial m_{H}}=0
$$

Thus, from Eq. (4.73), the derivative $\frac{\partial \omega_{2}\left(\mathbf{s}_{t}\right)}{\partial m_{\jmath}},(\jmath=1, \ldots, H)$, can be rewritten as 
follows,

$$
\frac{\partial \omega_{2}\left(\mathbf{s}_{t}\right)}{\partial m_{\jmath}}=\left\{\begin{array}{ccc}
\frac{2\left(s_{2}-m_{\jmath}\right)}{\sigma_{\jmath}^{2}} \omega_{2}\left(\mathbf{s}_{t}\right) & \text { if } \quad\left(\sigma_{\jmath}, m_{\jmath}\right) \in \Omega\left(\omega_{2}\right), \\
0 & \text { if } \quad\left(\sigma_{\jmath}, m_{\jmath}\right) \notin \Omega\left(\omega_{2}\right) .
\end{array}\right.
$$

We can rewrite Eq. (4.74) as follows,

$$
\frac{\partial \omega_{2}\left(\mathbf{s}_{t}\right)}{\partial m_{\jmath}}=\xi_{\jmath, 2} \frac{2\left(s_{\imath}-m_{\jmath}\right)}{\sigma_{\jmath}^{2}} \omega_{2}\left(\mathbf{s}_{t}\right)
$$

where $s_{\imath}$ is defined as in Eq. (4.50), and $\xi_{\jmath, 2}$ is defined as in Eq. (4.58).

Similarly, we can calculate the terms $\frac{\partial \omega_{3}\left(\mathbf{s}_{t}\right)}{\partial m_{\jmath}}, \frac{\partial \omega_{4}\left(\mathbf{s}_{t}\right)}{\partial m_{\jmath}}, \ldots$, and $\frac{\partial \omega_{L}\left(\mathbf{s}_{t}\right)}{\partial m_{\jmath}}$. Thus, Eq. (4.65) can be rewritten as follows,

$$
\frac{\partial \omega_{l}\left(\mathbf{s}_{t}\right)}{\partial m_{\jmath}}=\left[\xi_{\jmath, 1} \frac{2\left(s_{\imath}-m_{\jmath}\right)}{\sigma_{\jmath}^{2}} \omega_{1}\left(\mathbf{s}_{t}\right) \quad \xi_{\jmath, 2} \frac{2\left(s_{\imath}-m_{\jmath}\right)}{\sigma_{\jmath}^{2}} \omega_{2}\left(\mathbf{s}_{t}\right) \quad . \quad \cdot \quad \xi_{\jmath, L} \frac{2\left(s_{\imath}-m_{\jmath}\right)}{\sigma_{\jmath}^{2}} \omega_{L}\left(\mathbf{s}_{t}\right)\right]^{T},
$$

where $\xi_{\jmath, l}$ is defined as in Eq. (4.60).

From Eq. (4.44) and Eq. (4.76), the derivative $\frac{\partial V_{t}\left(\mathbf{s}_{t}\right)}{\partial m_{\jmath}}$ in Eq. (4.64) is calculated as follows,

$$
\frac{\partial V_{t}\left(\mathbf{s}_{t}\right)}{\partial m_{\jmath}}=\frac{2\left(s_{\imath}-m_{\jmath}\right)}{\sigma_{\jmath}^{2}} \times \sum_{l=1}^{L} \xi_{\jmath} \frac{k_{l}-V_{t}\left(\mathbf{s}_{t}\right)}{\sum_{l} \omega_{l}\left(\mathbf{s}_{t}\right)} \omega_{l}\left(\mathbf{s}_{t}\right)
$$

Similarly, we can calculate the derivative $\frac{\partial V_{t}\left(\mathbf{s}_{t+1}\right)}{\partial m_{\jmath}}$ as follows,

$$
\frac{\partial V_{t}\left(\mathbf{s}_{t+1}\right)}{\partial m_{\jmath}}=\frac{2\left(s_{\imath}^{\prime}-m_{\jmath}\right)}{\sigma_{\jmath}^{2}} \times \sum_{l=1}^{L} \xi_{\jmath} l \frac{k_{l}-V_{t}\left(\mathbf{s}_{t+1}\right)}{\sum_{l} \omega_{l}\left(\mathbf{s}_{t+1}\right)} \omega_{l}\left(\mathbf{s}_{t+1}\right) .
$$

Hence, From Eq. (4.38), Eq. (4.61), Eq. (4.62), Eq. (4.77) and Eq. (4.78), the input 
parameters $m_{\jmath}$ and $\sigma_{\jmath}$ of each critic are updated at each time step as follows,

$$
\begin{aligned}
& \sigma_{\jmath, t+1}=\sigma_{\jmath, t}-\rho^{C}\left[r_{t}+\zeta V_{t}\left(\mathbf{s}_{t+1}\right)-V_{t}\left(\mathbf{s}_{t}\right)\right] \cdot\left[\zeta \frac{\partial V_{t}\left(\mathbf{s}_{t+1}\right)}{\partial \sigma_{\jmath, t}}-\frac{\partial V_{t}\left(\mathbf{s}_{t}\right)}{\partial \sigma_{\jmath, t}}\right] \\
& m_{\jmath, t+1}=m_{\jmath, t}-\rho^{C}\left[r_{t}+\zeta V_{t}\left(\mathbf{s}_{t+1}\right)-V_{t}\left(\mathbf{s}_{t}\right)\right] \cdot\left[\zeta \frac{\partial V_{t}\left(\mathbf{s}_{t+1}\right)}{\partial m_{\jmath, t}}-\frac{\partial V_{t}\left(\mathbf{s}_{t}\right)}{\partial m_{\jmath, t}}\right] .
\end{aligned}
$$

Similarly, From Eq. (4.38), Eq. (4.39), Eq. (4.40), the output parameters $k_{l}$ of each critic are updated at each time step as follows,

$$
k_{l, t+1}=k_{l, t}-\rho^{C}\left[r_{t}+\zeta V_{t}\left(\mathbf{s}_{t+1}\right)-V_{t}\left(\mathbf{s}_{t}\right)\right] \cdot\left[\zeta \frac{\partial V_{t}\left(\mathbf{s}_{t+1}\right)}{\partial k_{l, t}}-\frac{\partial V_{t}\left(\mathbf{s}_{t}\right)}{\partial k_{l, t}}\right] .
$$

\subsubsection{Adaptation Rules for the Actor}

The input and the output parameters of the actor, $\psi^{A}$, are updated as follows, [100],

$$
\psi_{t+1}^{A}=\psi_{t}^{A}+\rho^{A} \Delta_{t} \frac{\partial u_{t}}{\partial \psi_{t}^{A}}\left[\frac{u_{c}-u_{t}}{\sigma_{n}}\right]
$$

where $\rho^{A}$ is a learning rate for the actor parameters, $u_{c}$ is the output of the actor with a random Gaussian noise. The derivatives of the output of the FLC (the actor), $u_{t}$, with respect to the input and output parameters of the FLC can be calculated by replacing $V_{t}\left(\mathbf{s}_{t}\right)$ with $u_{t}$ in Eq. (4.39), Eq. (4.61) and Eq. (4.77) as follows,

$$
\frac{\partial u_{t}}{\partial k_{l}}=\Phi_{l}\left(\mathbf{s}_{t}\right)
$$




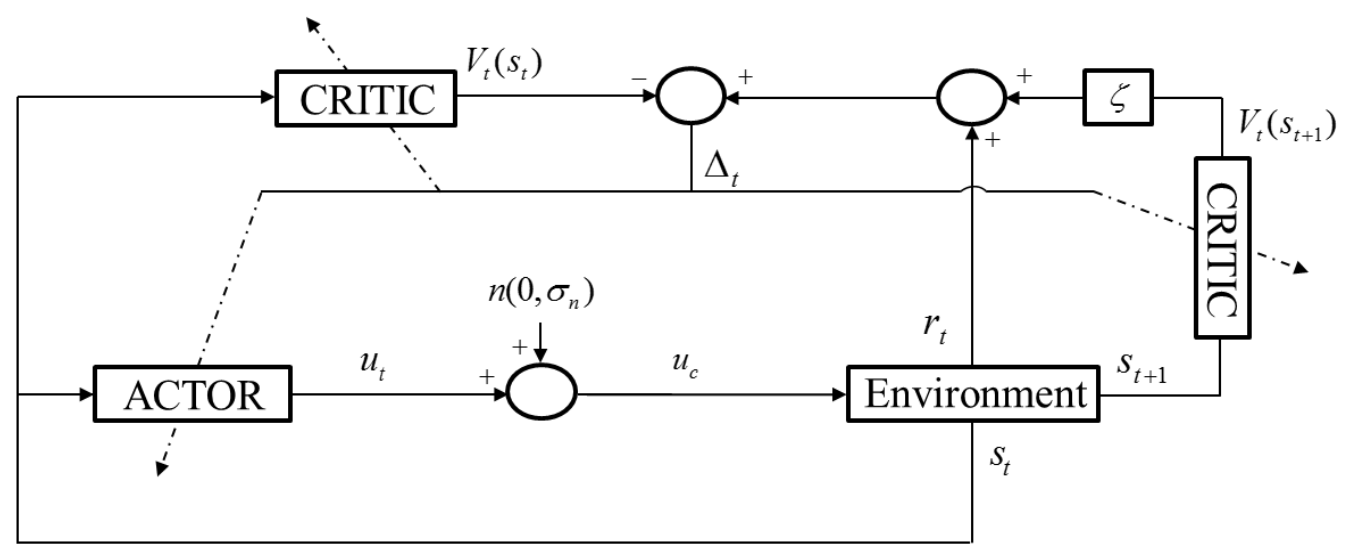

Figure 4.3: The proposed RGFACL algorithm.

$$
\begin{aligned}
\frac{\partial u_{t}}{\partial \sigma_{\jmath}} & =\frac{2\left(s_{\imath}-m_{\jmath}\right)^{2}}{\sigma_{\jmath}^{3}} \times \sum_{l=1}^{L} \xi_{\jmath} l \frac{k_{l}-u_{t}}{\sum_{l} \omega_{l}\left(\mathbf{s}_{t}\right)} \omega_{l}\left(\mathbf{s}_{t}\right), \\
\frac{\partial u_{t}}{\partial m_{\jmath}} & =\frac{2\left(s_{\imath}-m_{\jmath}\right)}{\sigma_{\jmath}^{2}} \times \sum_{l=1}^{L} \xi_{\jmath, l} \frac{k_{l}-u_{t}}{\sum_{l} \omega_{l}\left(\mathbf{s}_{t}\right)} \omega_{l}\left(\mathbf{s}_{t}\right) .
\end{aligned}
$$

Hence, From Eq. (4.82), Eq. (4.84) and Eq. (4.85), the input parameters $\sigma_{\jmath}$ and $m_{\text {ر }}$ of the actor (FLC) are updated at each time step as follows,

$$
\begin{gathered}
\sigma_{\jmath, t+1}=\sigma_{\jmath, t}+\rho^{A}\left[r_{t}+\zeta V_{t}\left(\mathbf{s}_{t+1}\right)-V_{t}\left(\mathbf{s}_{t}\right)\right] \frac{\partial u_{t}}{\partial \sigma_{\jmath, t}}\left[\frac{u_{c}-u_{t}}{\sigma_{n}}\right], \\
m_{\jmath, t+1}=m_{\jmath, t}+\rho^{A}\left[r_{t}+\zeta V_{t}\left(\mathbf{s}_{t+1}\right)-V_{t}\left(\mathbf{s}_{t}\right)\right] \frac{\partial u_{t}}{\partial m_{\jmath, t}}\left[\frac{u_{c}-u_{t}}{\sigma_{n}}\right] .
\end{gathered}
$$

Similarly, From Eq. (4.82) and Eq. (4.83), the output parameters $k_{l}$ of the actor (FLC) are updated at each time step as follows,

$$
k_{l, t+1}=k_{l, t}+\rho^{A}\left[r_{t}+\zeta V_{t}\left(\mathbf{s}_{t+1}\right)-V_{t}\left(\mathbf{s}_{t}\right)\right] \frac{\partial u_{t}}{\partial k_{l, t}}\left[\frac{u_{c}-u_{t}}{\sigma_{n}}\right] .
$$

The proposed RGFACL algorithm is given in Algorithm 6. 


\begin{abstract}
Algorithm 6 The Proposed Residual Gradient Fuzzy Actor Critic Learning (RGFACL) Algorithm.
\end{abstract}

(1) Initialize:

(a) the input and the output parameters of the critic, $\psi^{C}$.

(b) the input and the output parameters of the actor, $\psi^{A}$.

(2) For each EPISODE do:

(3) Update the learning rates $\rho^{C}$ and $\rho^{A}$ of the critic and actor, respectively.

(4) Initialize the position of the pursuer at $\left(x_{p}, y_{p}\right)=0$ and the position of the evader randomly at $\left(x_{e}, y_{e}\right)$, and then calculate the initial state $\mathbf{s}_{t}$.

(5) For each ITERATION do:

(6) Calculate the output of the actor, $u_{t}$, at the state $\mathbf{s}_{t}$ by using Eq. (4.14) and then calculate the output $u_{c}=u_{t}+n\left(0, \sigma_{n}\right)$.

(7) Calculate the output of the first critic, $V_{t}\left(\mathbf{s}_{t}\right)$, at the state $\mathbf{s}_{t}$ by using Eq. (4.14).

(8) Perform the action $u_{c}$ and observe the next state $\mathbf{s}_{t+1}$ and the reward $r_{t}$.

(9) Calculate the output of the second critic, $V_{t}\left(\mathbf{s}_{t+1}\right)$, at the next state $\mathbf{s}_{t+1}$ by using Eq. (4.14).

(10) Calculate the temporal difference error, $\Delta_{t}$, by using Eq. (4.34).

(11) Update the input and the output parameters of each critic, $\psi^{C}$, by using Eq. (4.79), Eq. (4.80) and Eq. (4.81).

(12) Update the input and the output parameters of the actor, $\psi^{A}$, based on Eq. (4.86), Eq. (4.87) and Eq. (4.88).

(13) Set $\mathbf{s}_{t} \leftarrow \mathbf{s}_{t+1}$

(14) Check Termination Condition.

(15) end for loop (ITERATION).

(16) end for loop (EPISODE).

\title{
4.7 Simulation and Results
}

We evaluate the proposed RGFACL algorithm, the FACL algorithm and the QLFIS algorithm on three different pursuit-evasion games. In the first game, the evader is following a simple control strategy, whereas the pursuer is learning its control strategy to capture the evader in minimum time. In the second game, it is also only the pursuer that is learning. However, the evader in this game is following an intelligent control strategy that exploits the advantage of the maneuverability of the evader. In the third game, we make both the pursuer and the evader learn their control strategies. In multi-robot learning systems, each robot will try to learn its 
control strategy by interacting with the other robot which is also learning at the same time. Therefore, the complexity of the system will increase as the learning in a multi-robot system is considered as a problem of a "moving target" [9]. In the problem of a moving target, the best-response policy of each learning robot may keep changing during learning until each learning robot adopts an equilibrium policy. It is important to mention here that the pursuer, in all games, is assumed to not know the dynamics of the evader nor its control strategy.

We use the same learning and exploration rates for all algorithms when they are applied to the same game. Those rates are chosen to be similar to those used in [100]. We define the angle difference between the direction of the pursuer and the line-ofsight (LoS) vector of the pursuer to the evader by $\delta_{p}$. In all games, we define the state $\mathbf{s}_{t}$ for the pursuer by the two input variables which are the pursuer angle difference $\delta_{p}$ and its derivative $\dot{\delta}_{p}$. In the third game, we define the state $\mathbf{s}_{t}$ for the evader by the two input variables which are the evader angle difference $\delta_{e}$ and its derivative $\dot{\delta}_{e}$. Three Gaussian membership functions (MFs) are used to define the fuzzy sets of each input. Thus, eash FIS has two inputs $(n=2)$ with three MFs for each input $(h=3)$. That is, the input parameters of each FIS are $\sigma_{\jmath}$ and $m_{\jmath}$, where $(\jmath=1, \ldots, H$ and $H=n \times h=6)$. In addition, each FIS has nine rules $\left(L=h^{n}=9\right)$. That is, the output parameters of each FIS are $k_{l}$, where $(l=1, \ldots, L)$. The parameters of the MFs of each input $\Omega\left(s_{\imath}\right),(\imath=1,2)$, defined by Eq. (4.13) can be given as follows,

$$
\begin{aligned}
& \Omega\left(s_{1}\right)=\left\{\left(\sigma_{1}, m_{1}\right),\left(\sigma_{2}, m_{2}\right),\left(\sigma_{3}, m_{3}\right)\right\} \\
& \Omega\left(s_{2}\right)=\left\{\left(\sigma_{4}, m_{4}\right),\left(\sigma_{5}, m_{5}\right),\left(\sigma_{6}, m_{6}\right)\right\} .
\end{aligned}
$$

On the other hand, the set of the parameters of each firing strength of each rule in 
each FIS, $\Omega\left(\omega_{l}\right)$, defined by Eq. (4.17) is given as follows,

$$
\begin{aligned}
& \Omega\left(\omega_{1}\right)=\left\{\left(\sigma_{1}, m_{1}\right),\left(\sigma_{4}, m_{4}\right)\right\}, \\
& \Omega\left(\omega_{2}\right)=\left\{\left(\sigma_{1}, m_{1}\right),\left(\sigma_{5}, m_{5}\right)\right\}, \\
& \Omega\left(\omega_{3}\right)=\left\{\left(\sigma_{1}, m_{1}\right),\left(\sigma_{6}, m_{6}\right)\right\}, \\
& \Omega\left(\omega_{4}\right)=\left\{\left(\sigma_{2}, m_{2}\right),\left(\sigma_{4}, m_{4}\right)\right\}, \\
& \Omega\left(\omega_{5}\right)=\left\{\left(\sigma_{2}, m_{2}\right),\left(\sigma_{5}, m_{5}\right)\right\}, \\
& \Omega\left(\omega_{6}\right)=\left\{\left(\sigma_{2}, m_{2}\right),\left(\sigma_{6}, m_{6}\right)\right\}, \\
& \Omega\left(\omega_{7}\right)=\left\{\left(\sigma_{3}, m_{3}\right),\left(\sigma_{4}, m_{4}\right)\right\}, \\
& \Omega\left(\omega_{8}\right)=\left\{\left(\sigma_{3}, m_{3}\right),\left(\sigma_{5}, m_{5}\right)\right\}, \\
& \Omega\left(\omega_{9}\right)=\left\{\left(\sigma_{3}, m_{3}\right),\left(\sigma_{6}, m_{6}\right)\right\} .
\end{aligned}
$$

The term $\xi_{\jmath, l}$ defined by Eq. (4.60) can be calculated based on the following matrix,

$$
\xi=\left[\begin{array}{lllllllll}
1 & 1 & 1 & 0 & 0 & 0 & 0 & 0 & 0 \\
0 & 0 & 0 & 1 & 1 & 1 & 0 & 0 & 0 \\
0 & 0 & 0 & 0 & 0 & 0 & 1 & 1 & 1 \\
1 & 0 & 0 & 1 & 0 & 0 & 1 & 0 & 0 \\
0 & 1 & 0 & 0 & 1 & 0 & 0 & 1 & 0 \\
0 & 0 & 1 & 0 & 0 & 1 & 0 & 0 & 1
\end{array}\right]_{6 \times 9}
$$


In all games, we assume that the pursuer is faster than the evader, and the evader is more maneuverable than the pursuer. In addition, the pursuer is assumed to not know the dynamics of the evader nor its control strategy. The only information the pursuer knows about the evader is the position (location) of the evader. The parameters of the pursuer are set as follows, $V_{p}=2.0 \mathrm{~m} / \mathrm{s}, L_{p}=0.3 \mathrm{~m}$ and $u_{p} \in[-0.5,0.5]$. The pursuer starts its motion from the position $\left(x_{p}, y_{p}\right)=(0,0)$ with an initial orientation $\theta_{p}=0$. On the other hand, the parameters of the evader are set up as follows, $V_{e}=1 \mathrm{~m} / \mathrm{s}$, $L_{e}=0.3 \mathrm{~m}$ and $u_{e} \in[-1.0,1.0]$. The evader starts its motion from a random position at each episode with an initial orientation $\theta_{e}=0$. The sampling time is defined as $T=0.05 \mathrm{~s}$, whereas the capture radius is defined as $d_{c}=0.1 \mathrm{~m}$.

\subsubsection{Pursuit-Evasion Game 1}

In this game, the evader is following a simple control strategy defined by Eq. (4.30). On the other hand, the pursuer is learning its control strategy with the proposed RGFACL algorithm. We compare our results with the results obtained when the pursuer is following the classical control strategy defined by Eq. (4.28) and Eq. (4.29). We also compare our results with the results obtained when the pursuer is learning its control strategy by the FACL and the QLFIS algorithms. We define the number of episodes in this game as 200 and the number of steps (in each episode) as 600. For each algorithm (the FACL, the QLFIS and the proposed RGFACL algorithms), we ran this game 20 times and we averaged the capture time of the evader over this number of trials.

Table 4.1 shows the time that the pursuer takes to capture the evader when the evader is following a simple control strategy and starts its motion from different initial positions. The table shows the capture time of the evader when the pursuer is following the classical control strategy and when the pursuer is learning its control 
Table 4.1: The time (in seconds) that the pursuer trained by each algorithm takes to capture an evader that follows a simple control strategy. The number of episodes here is 200. The evader starts motion at different positions.

\begin{tabular}{|l||c|c|c|c|}
\hline \multicolumn{1}{|c||}{ Evader } & $(-5,9)$ & $(-10,-6)$ & $(7,4)$ & $(5,-10)$ \\
\hline \hline Clasorithm & & & & \\
\hline The proposed RGFACL & 10.70 & 12.40 & 8.05 & 11.20 \\
\hline QLFIS & 10.90 & 12.45 & 8.05 & 11.25 \\
\hline FACL & 11.50 & 13.50 & 8.05 & 11.35 \\
\hline
\end{tabular}

strategy by the FACL algorithm, the QLFIS algorithm and the proposed RGFACL algorithm. From Table 4.1, we can see that the capture time of the evader when the pursuer learns its control strategy by the proposed RGFACL algorithm is very close to the capture time of the evader when the pursuer follows the classical control strategy. This shows that the proposed RGFACL algorithm achieves the performance of the classical control.

\subsubsection{Pursuit-Evasion Game 2}

In this game, the evader is following the control strategy defined by Eq. (4.30) and Eq. (4.31) with the advantage of using its higher maneuverability. On the other hand, the pursuer in this game is learning its control strategy with the proposed RGFACL algorithm. Similar to game 1, we compare our results obtained when the pursuer is learning by the proposed RGFACL algorithm with the results obtained when the pursuer is following the classical control strategy defined by Eq. (4.28) and Eq. (4.29). We also compare our results with the results obtained when the pursuer is learning its control strategy by the FACL and the QLFIS algorithms. In [100], it is assumed that the velocity of the pursuer and evader are governed by their steering 
angles so that the pursuer and evader can avoid slips during turning. This constraint will make the evader slow down its speed whenever the evader makes a turn. This will make it easy for the pursuer to capture the evader. Our objective is to see how the proposed algorithm and the other studied algorithms will behave when the evader makes use of the advantage of the maneuverability without any velocity constraints. Thus, in this chapter, we take this velocity constraints out so that both the pursuer and the evader can make fast turns without any velocity constraints. In this game, we use two different numbers for the episodes (200 and 1000), whereas the number of steps (in each episode) is set as 3000. For each algorithm (the FACL, the QLFIS and the proposed RGFACL algorithms), we ran this game 20 times and, then, averaged the capture time of the evader over this number of trials.

Table 4.2 and Table 4.3 show the time that the pursuer takes to capture the evader when the evader is following the control strategy defined by Eq. (4.30) and Eq. (4.31) with the advantage of using its higher maneuverability. The number of episodes used here is 200 for Table 4.2 and 1000 for Table 4.3. The tables show that the pursuer fails to capture the evader when the pursuer is following the classical control strategy and when learning by the FACL algorithm. Table 4.2 shows that the pursuer succeeds to capture the evader in all 20 trials only when the pursuer is learning by the proposed RGFACL algorithm. When learning by the QLFIS algorithm, the pursuer succeeds to capture the evader only in $20 \%$ of the 20 trials. On the other hand, Table 4.3 shows that the pursuer always succeeds to capture the evader only when the pursuer is learning with the proposed RGFACL algorithm. However, when learning with the QLFIS algorithm, the pursuer succeeds to capture the evader only in $50 \%$ of the 20 trials. Table 4.2 and Table 4.3 show that the proposed RGFACL algorithm outperforms the FACL and the QLFIS algorithms. This is because the 
Table 4.2: The time (in seconds) that the pursuer trained by each algorithm takes to capture an evader that follows an intelligent control strategy. The number of episodes here is 200 . The evader starts motion at different positions.

\begin{tabular}{|l||c|c|c|c|}
\hline \multicolumn{1}{|c||}{ Evader } & $(-9,7)$ & $(-7,-10)$ & $(6,9)$ & $(3,-9)$ \\
\hline \hline Classical strategy & No Capture & No Capture & No Capture & No Capture \\
\hline The proposed RGFACL & 13.05 & 14.30 & 11.65 & 11.15 \\
& $100 \%$ & $100 \%$ & $100 \%$ & $100 \%$ \\
\hline QLFIS & 23.20 & 23.55 & 25.45 & 21.35 \\
& $20 \%$ & $20 \%$ & $20 \%$ & $20 \%$ \\
\hline \multirow{2}{*}{ FACL } & No Capture & No Capture & No Capture & No Capture \\
\hline
\end{tabular}

Table 4.3: The time (in seconds) that the pursuer trained by each algorithm takes to capture an evader that follows an intelligent control strategy. The number of episodes here is 1000 . The evader starts motion at different positions.

\begin{tabular}{|l||c|c|c|c|}
\hline \multicolumn{1}{|c||}{ Evader } & $(-9,7)$ & $(-7,-10)$ & $(6,9)$ & $(3,-9)$ \\
\hline \hline Classical strategy & No Capture & No Capture & No Capture & No Capture \\
\hline The proposed RGFACL & 12.70 & 13.15 & 11.30 & 10.90 \\
& $100 \%$ & $100 \%$ & $100 \%$ & $100 \%$ \\
\hline QLFIS & 20.25 & 21.60 & 19.60 & 19.20 \\
& $50 \%$ & $50 \%$ & $50 \%$ & $50 \%$ \\
\hline FACL & No Capture & No Capture & No Capture & No Capture \\
\hline
\end{tabular}

pursuer using the proposed RGFACL algorithm to learn its control strategy always succeeds to capture the evader in less time as well as in a less number of episodes.

\subsubsection{Pursuit-Evasion Game 3}

Unlike game 1 and game 2, both the evader and the pursuer are learning their control strategies in this game. In multi-robot learning systems, each robot will try to learn its control strategy by interacting with the other robot which is also learning its 
control strategy at the same time. Thus, the complexity of the system will increase in this game as the learning in a multi-robot system is considered as a problem of a "moving target" [9]. We compare the results obtained by the proposed algorithm with the results obtained by the FACL and QLFIS algorithms. Unlike the first two pursuit-evasion games, we do not use the capture time of the evader as a criterion in our comparison in this game. This is because both the pursuer and the evader are learning. That is, a small capture time by the pursuer learning its control strategy by one of the learning algorithms can have two different indications; the first one is that the learning algorithm is working well as the pursuer succeeds to capture the evader quickly. The second indication, on the other hand, is that the learning algorithm is not working properly as the evader does not learn how to escape from the pursuer. Therefore, we compare the paths of the pursuer and the evader learning their control strategies by the learning algorithms with the paths of the pursuer and the evader following the classical control strategy defined by Eq. (4.28) and Eq. (4.29).

In game 3 , the pursuer starts its motion from the position $\left(x_{p}, y_{p}\right)=(0,0)$ with an initial orientation $\theta_{p}=0$. On the other hand, the evader starts its motion from a random position at each episode with an initial orientation $\theta_{e}=0$. We run game 3 twice. In the first run, we set the number of the episodes in this game to 200, whereas the number of steps in each episode is set to 3000. The results of the first run are shown in Fig. (4.4) to Fig. (4.6) when the evader starts its motion from the position $\left(x_{e}, y_{e}\right)=(-10,-10)$. These figures show the paths of the pursuer and the evader (starred lines) when learning by the FACL, the QLFIS, and the proposed RGFACL algorithms, respectively. The paths of the pursuer and the evader following the classical control strategy are also shown in the figures (dotted lines). The results of the first run show that the proposed RGFACL algorithm outperforms the FACL and the QLFIS algorithms as the performance of the proposed algorithm is close to 


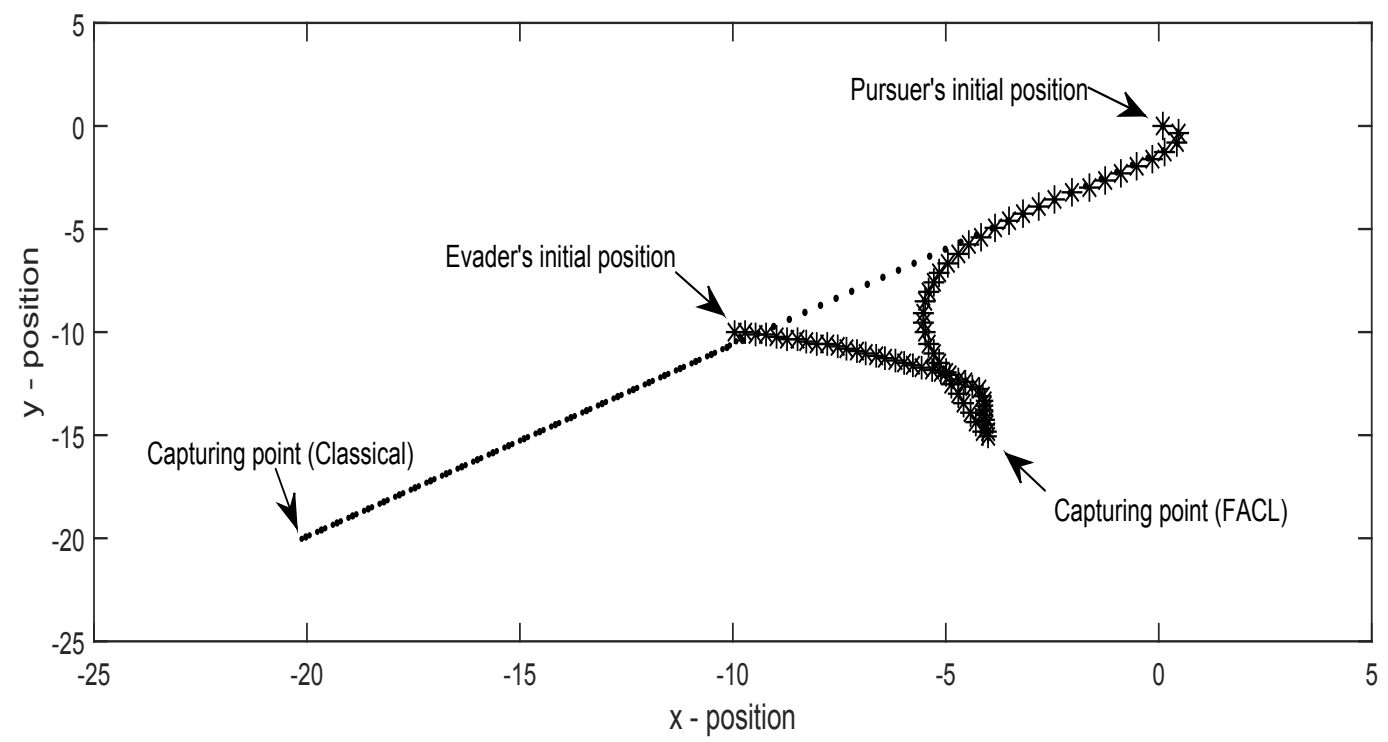

Figure 4.4: The paths of the pursuer and the evader when learning by the FACL algorithm proposed in [99] (starred lines) against the paths of the pursuer and the evader when following the classical strategy defined in Eq. (4.28) and Eq. (4.29) (dotted lines). The number of episodes used here is 200.

the performance of the classical control strategy. In the second run of game 3, we set the number of the episodes in this game to 500, whereas the number of steps in each episode is set to 3000. The results of the second run are shown in Fig. (4.7) to Fig. (4.9) when the evader starts its motion from the position $\left(x_{e}, y_{e}\right)=(-10,-10)$. The figures show that the performance of the proposed RGFACL algorithm and the performance of the QLFIS algorithm are close to the performance of the classical control strategy and both algorithms outperform the FACL algorithm.

\subsection{Summary and Discussion}

In this chapter, we propose a new fuzzy reinforcement learning algorithm for differential games that have continuous state and action spaces. The proposed algorithm uses FISs as FASs; an actor (fuzzy logic controller, FLC) and a critic. The proposed 


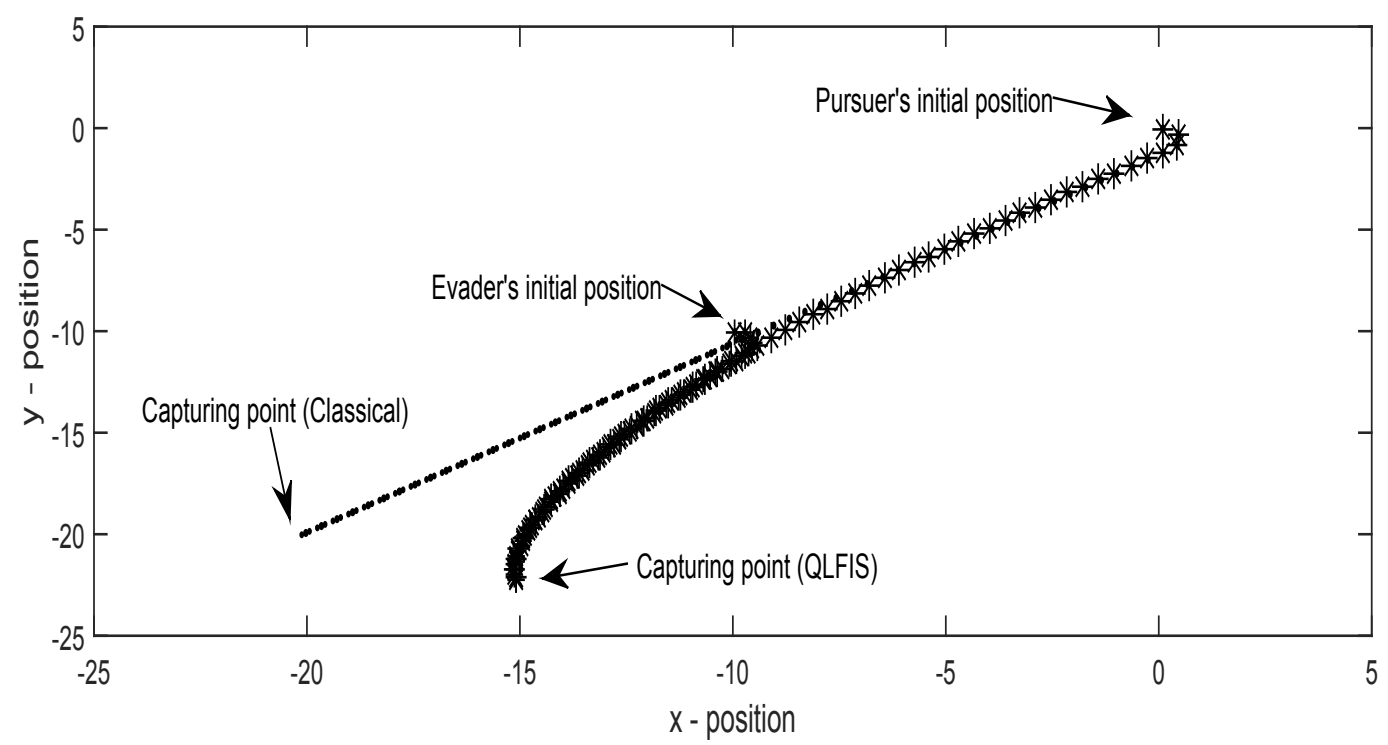

Figure 4.5: The paths of the pursuer and the evader when learning by the QLFIS algorithm proposed in [100] (starred lines) against the paths of the pursuer and the evader when following the classical strategy defined in Eq. (4.28) and Eq. (4.29) (dotted lines). The number of episodes used here is 200.

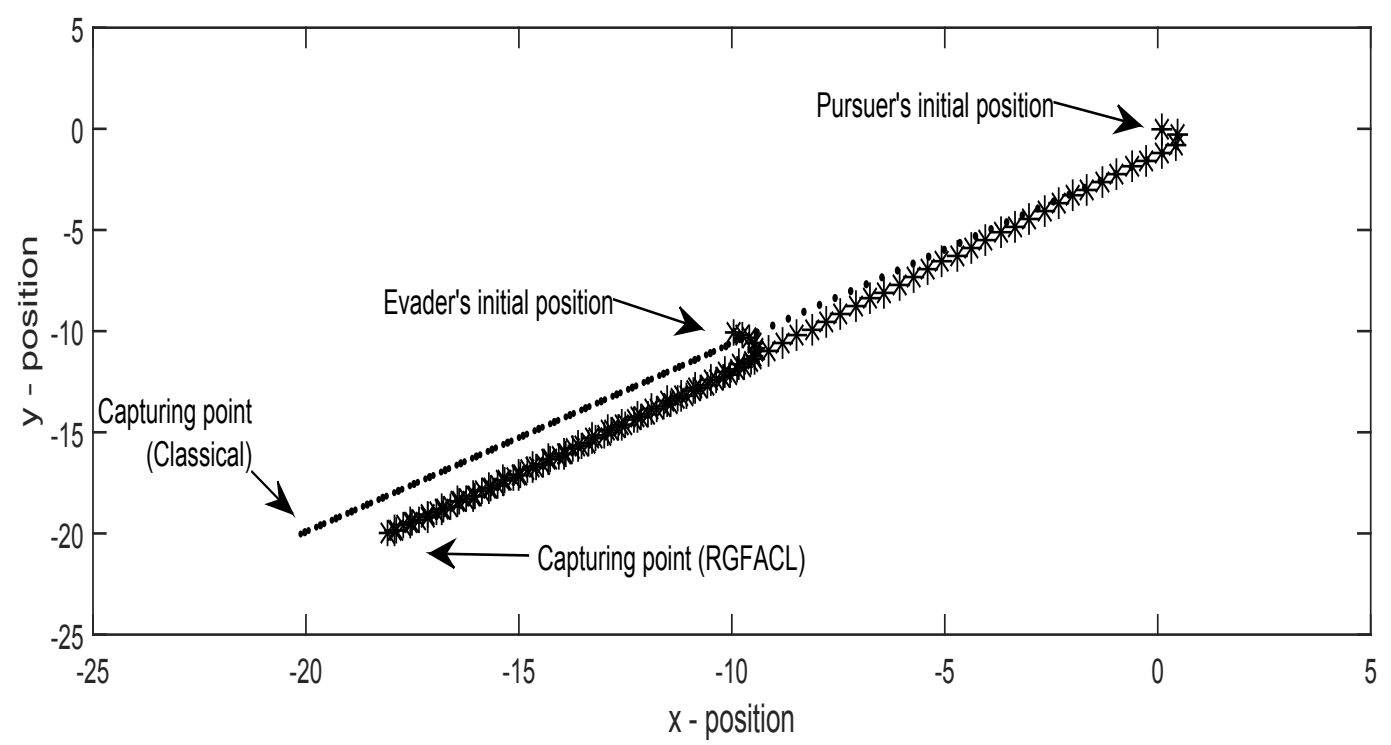

Figure 4.6: The paths of the pursuer and the evader when learning by the proposed RGFACL algorithm (starred lines) against the paths of the pursuer and the evader when following the classical strategy defined in Eq. (4.28) and Eq. (4.29) (dotted lines). The number of episodes used here is 200. 


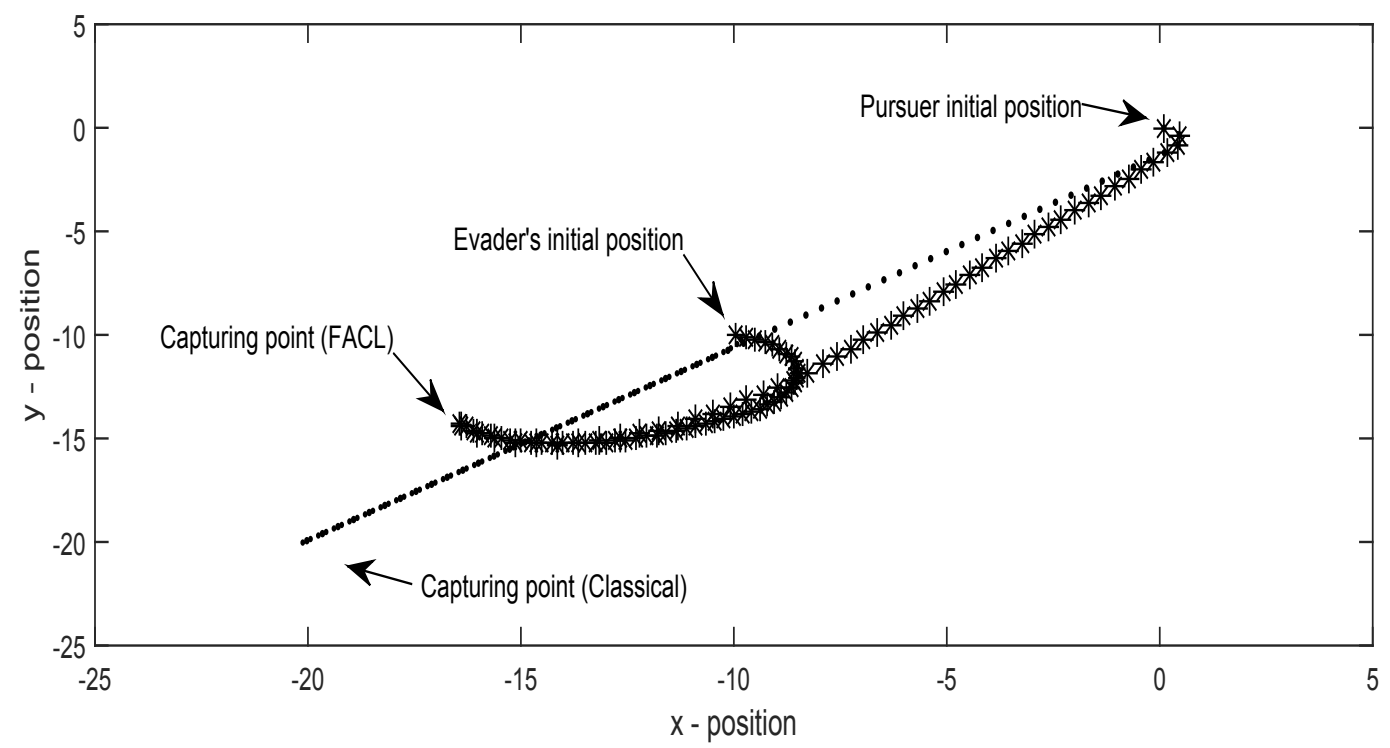

Figure 4.7: The paths of the pursuer and the evader when learning by the FACL algorithm proposed in [99] (starred lines) against the paths of the pursuer and the evader when following the classical strategy defined in Eq. (4.28) and Eq. (4.29) (dotted lines). The number of episodes used here is 500.

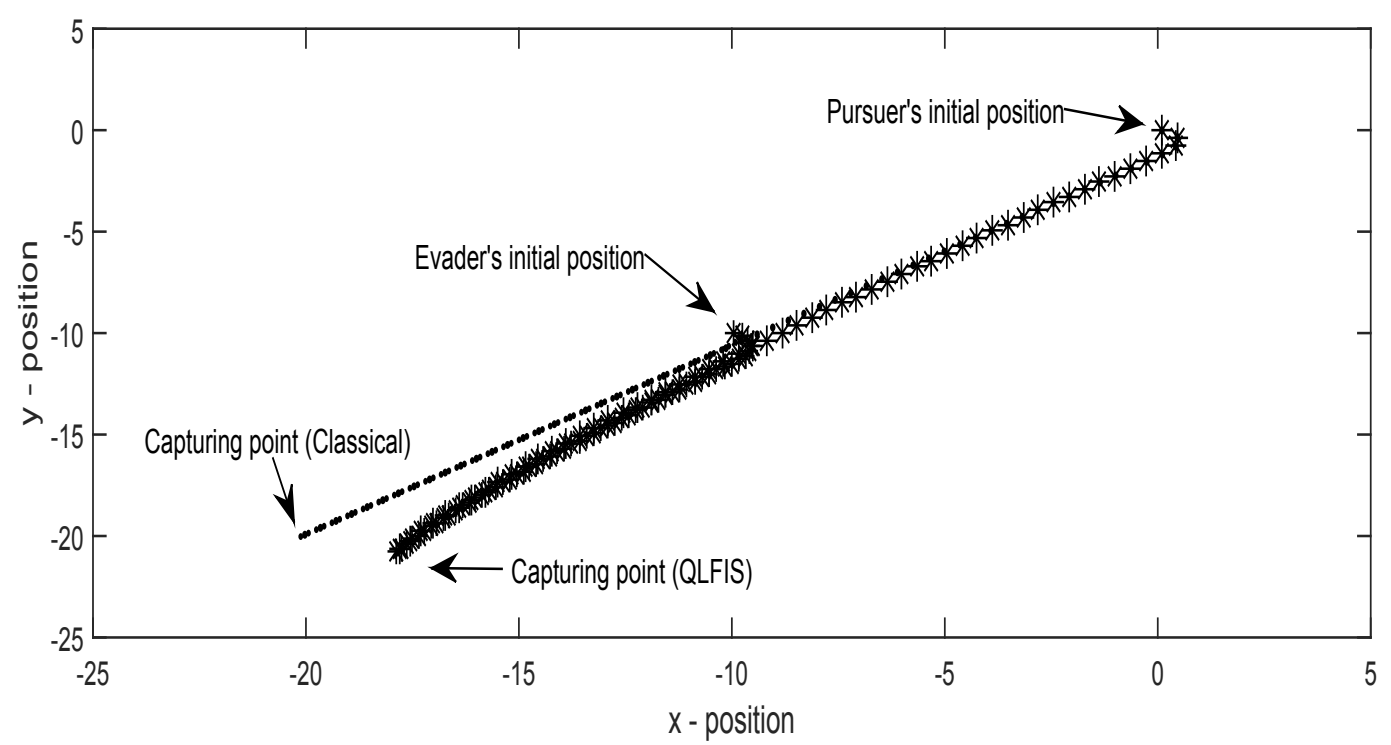

Figure 4.8: The paths of the pursuer and the evader when learning by the QLFIS algorithm proposed in [100] (starred lines) against the paths of the pursuer and the evader when following the classical strategy defined in Eq. (4.28) and Eq. (4.29) (dotted lines). The number of episodes used here is 500. 


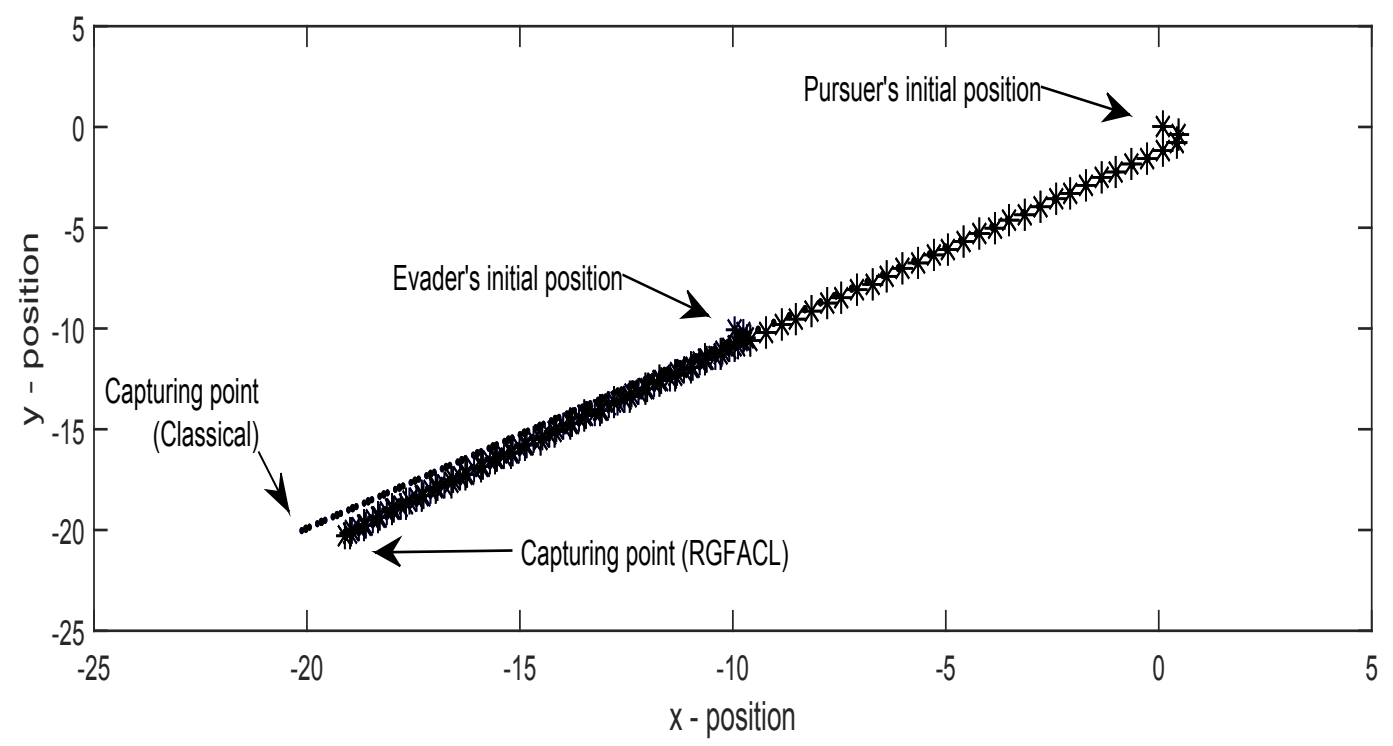

Figure 4.9: The paths of the pursuer and the evader when learning by the proposed RGFACL algorithm (starred lines) against the paths of the pursuer and the evader when following the classical strategy defined in Eq. (4.28) and Eq. (4.29) (dotted lines). The number of episodes used here is 500.

algorithm tunes the input and the output parameters of its FASs (the actor and the critic) differently from the tuning mechanisms used in the algorithms proposed in in the literature. The proposed algorithm uses the residual gradient value iteration algorithm as a mechanism in tuning the parameters of its FASs; whereas the algorithms proposed in the literature use the direct algorithms in their mechanisms to tune the parameters of their FASs. The proposed algorithm is called the residual gradient fuzzy actor critic learning (RGFACL) algorithm. It has been shown in the literature that the residual gradient algorithms are superior to the direct algorithms as the residual gradient algorithms are always guaranteed to converge, whereas the direct algorithms may not converge to an answer in some cases. The proposed algorithm is used to learn three different pursuit-evasion games. We start with the game where the pursuer learns its control strategy and the evader follows a simple control strategy. In the second game, the pursuer learns its control strategy and the evader 
follows an intelligent control strategy that exploits the advantage of higher maneuverability. In the third game, we increase the complexity of the system by making both the pursuer and the evader learn their control strategies. Simulation results show that the proposed RGFACL algorithm outperforms the FACL and the QLFIS algorithms in terms of performance and the learning time when they all are used to learn the pursuit-evasion games considered in this chapter. 


\section{Chapter 5}

\section{A Fuzzy Reinforcement Learning Algorithm Using a Predictor for Pursuit-Evasion Games}

\section{$5.1 \quad$ Introduction}

A robot learning by any fuzzy reinforcement learning algorithm usually succeeds to perform its task when the trained robot is used in an environment that is similar to the environment the robot was trained on. However, in some cases, the performance of the trained robot will be degraded if the trained robot is used in an environment different from the one that the robot was trained on. In this case, the trained robot has to be retrained so that the robot can learn how to achieve its task in the new environment [137]. When fuzzy reinforcement learning algorithms are applied to real-world applications with environments different from the training environments, these algorithms will need time to relearn and adjust their performance. In some real-world applications, the time needed by these algorithms in the readjustment phase may affect the performance of these algorithms and lead to failure in achieving the tasks. 
In this chapter, we are interested in pursuit-evasion differential games since they are useful for many real-world applications including surveillance and tracking, search and rescue, locating and capturing hostile intruders, and localizing and neutralizing environmental threats [3]. Different fuzzy reinforcement learning algorithms [99-101] that can be used to solve the problem of the pursuit-evasion game have been discussed in the previous chapter. When using these learning algorithms in pursuit-evasion games, the pursuer is first trained in simulation so that the pursuer can learn its control strategy. When the training phase is finished and the pursuer learns its control strategy, the trained pursuer can then be used in a real-world pursuit-evasion game to capture the evader. However, in the real field, the environment may be different from the training environment. That is, the trained pursuer may be faced with a new and different evader with different parameters. Our concern is in the case when the trained pursuer faces a different unexpected evader. Would the trained pursuer be able to capture that unexpected evader? Therefore, we investigate a number of learning algorithms and see how quickly each algorithm adapts to the new different environment and whether or not the pursuer trained by each algorithm can capture the different evader. We also propose a new fuzzy reinforcement learning algorithm so that the pursuer trained by the proposed algorithm can capture the evader not only when the environment of the game is similar to the training environment but also when the environment of the game is different from the training environment. The proposed algorithm is published in [105]

The proposed algorithm is applied to different single pursuit-evasion differential games. These pursuit-evasion games have environments different from the training environment that the pursuer was trained on. That is, we train the pursuer in simulation. Then, the trained pursuer is used to capture the evader in these 
pursuit-evasion games, where the evaders in these games are different from the evader that was used in the training phase. We compare the proposed algorithm with the fuzzy reinforcement learning (FACL, QLFIS and RGFACL) algorithms that were used in the previous chapter. The simulation results show that the proposed algorithm outperforms the fuzzy reinforcement learning (FACL, QLFIS and RGFACL) algorithms in terms of the ability of the pursuer to capture the evader and the capture time when these algorithms are applied to such pursuit-evasion games.

\subsection{The Proposed Algorithm}

The proposed algorithm is a modified version of the RGFACL algorithm proposed in [101] and in the previous chapter. Different fuzzy learning algorithms that can be applied to pursuit-evasion differential games are proposed in the literature [99-101]. In this chapter, the proposed algorithm uses the RGFACL algorithm as the RGFACL algorithm is shown in [101] to be more robust and has a quicker convergence speed than the other (FACL and QLFIS) algorithms. At each time step, the proposed algorithm uses a Kalman filter to predict the next position of the evader at the next time step. The proposed algorithm is called the Kalman filter fuzzy actor critic learning (KFFACL) algorithm. The proposed algorithm provides a control strategy to the pursuer, in a pursuit-evasion game, so that the pursuer will be able to capture the evader even when the environment of the game is different from the training environment. The Kalman filter can integrate into the learning algorithm information about the current observed dynamics of the evader. Even when the real environment is different from the training environment, the Kalman filter adapts the learning algorithm to that difference and quickly enables the learning algorithm to adjust to the true observed behaviour of the evader. 


\subsubsection{A Kalman Filter Predictor}

We use a Kalman filter to estimate the future position of the evader at the next time step. We define the position and the velocity of the evader by a state vector as follows,

$$
\boldsymbol{x}(t)=\left[\begin{array}{c}
x_{e}(t) \\
\dot{x}_{e}(t) \\
y_{e}(t) \\
\dot{y}_{e}(t)
\end{array}\right] .
$$

The terms $x_{e}(t)$ and $y_{e}(t)$ in Eq. (5.1) represent the $x-y$ components of the position of the evader, whereas the terms $\dot{x}_{e}(t)$ and $\dot{y}_{e}(t)$ in Eq. (5.1) represent the $x-y$ components of the velocity of the evader.

We assume that the evader has a constant speed, $V_{e}$. We also assume that the control strategy of the evader is unknown. That is, the pursuer does not know what the evader will do. Thus, we assume that the evader is driven by a white noise. In other words, we simply assume that the evader is represented by a "dead reckoning" model and any maneuvers the evader makes will be modeled as a white noise. We make no assumptions about the true dynamics of the evader given by Eq. (4.27). Thus, the evader's model can be described as follows,

$$
\begin{aligned}
& \ddot{x}_{e}(t)=w_{x}(t), \\
& \ddot{y}_{e}(t)=w_{y}(t),
\end{aligned}
$$

where $w_{x}(t)$ and $w_{y}(t)$ represent zero-mean white Gaussian process noise with variances $\sigma_{w_{x}}^{2}$ and $\sigma_{w_{y}}^{2}$, respectively. 
Eq. (5.2) can be rewritten as follows,

$$
\dot{\boldsymbol{x}}(t)=\left[\begin{array}{cccc}
0 & 1 & 0 & 0 \\
0 & 0 & 0 & 0 \\
0 & 0 & 0 & 1 \\
0 & 0 & 0 & 0
\end{array}\right]\left[\begin{array}{l}
x_{e}(t) \\
\dot{x}_{e}(t) \\
y_{e}(t) \\
\dot{y}_{e}(t)
\end{array}\right]+\left[\begin{array}{ll}
0 & 0 \\
1 & 0 \\
0 & 0 \\
0 & 1
\end{array}\right]\left[\begin{array}{l}
w_{x}(t) \\
w_{y}(t)
\end{array}\right] .
$$

We assume that only the position components of the evader, $x_{e}(t)$ and $y_{e}(t)$, are measurable. Thus, we define the measurement model of the evader as follows,

$$
\boldsymbol{y}(t)=\left[\begin{array}{cccc}
1 & 0 & 0 & 0 \\
0 & 0 & 1 & 0
\end{array}\right]\left[\begin{array}{l}
x_{e}(t) \\
\dot{x}_{e}(t) \\
y_{e}(t) \\
\dot{y}_{e}(t)
\end{array}\right]+\left[\begin{array}{ll}
1 & 0 \\
0 & 1
\end{array}\right]\left[\begin{array}{l}
v_{x}(t) \\
v_{y}(t)
\end{array}\right],
$$

where $v_{x}(t)$ and $v_{y}(t)$ represent zero-mean white Gaussian measurement noise with variances $\sigma_{v_{x}}^{2}$ and $\sigma_{v_{y}}^{2}$, respectively.

The discrete-time system model of Eq. (5.3) and the discrete-time measurement model of Eq. (5.4) are given, when the sampling period is $T$, as follows,

$$
\begin{gathered}
\boldsymbol{x}(k+1)=F \boldsymbol{x}(k)+G w(k), \\
\boldsymbol{y}(k)=H \boldsymbol{x}(k)+D v(k) .
\end{gathered}
$$

The transition matrix $F$ and the matrix $H$ are given as in the following equations. 
On the other hand, the matrices $G$ and $D$ are not specified here as they are being dealt with as parts of the process noise covariance matrix $Q_{k f}$ and the observation noise covariance matrix $R_{k f}$ that will be defined later on in our simulation.

$$
\begin{aligned}
& F=\left[\begin{array}{llll}
1 & T & 0 & 0 \\
0 & 1 & 0 & 0 \\
0 & 0 & 1 & T \\
0 & 0 & 0 & 1
\end{array}\right] . \\
& H=\left[\begin{array}{llll}
1 & 0 & 0 & 0 \\
0 & 0 & 1 & 0
\end{array}\right] .
\end{aligned}
$$

Thus, the Kalman filter can be used now to predict the next position of the evader after selecting the proper process and measurement covariance matrices $Q_{k f}$ and $R_{k f}$. For more information about Kalman filter, the reader may refer to [49-51].

\subsubsection{The Proposed KFFACL Algorithm}

The proposed algorithm tunes the input and the output parameters of the FLC of the pursuer based on the next position of the evader predicted by a Kalman filter. Fig (5.1) shows a pursuit-evasion game with the predicted position of the evader. The position of the pursuer is defined as $\left(x_{p}, y_{p}\right)$, the position of the evader is defined as $\left(x_{e}, y_{e}\right)$, and the estimated future position of the evader at the next time step is defined as $\left(\hat{x_{e}}, \hat{y}_{e}\right)$. Fig $(5.1)$ also shows how the proposed algorithm defines $\delta_{p}$, the angle difference between the directions of the pursuer and its line-of-sight (LoS) vector to the evader at its real location $\left(x_{e}, y_{e}\right)$. In addition, Fig (5.1) shows how to define $\hat{\delta_{p}}$, the predicted angle difference between the directions of the pursuer and its LoS 


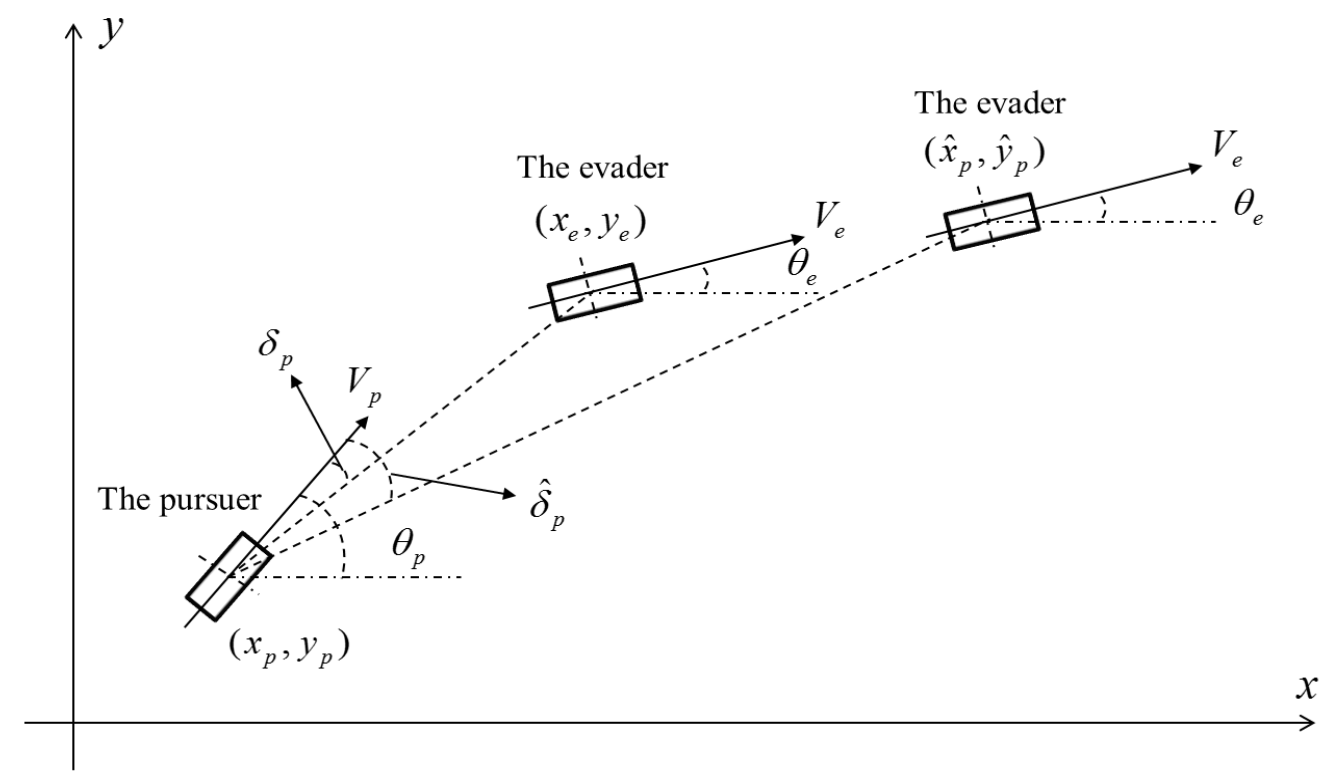

Figure 5.1: Pursuit-evasion model with the predicted position of the evader.

vector to the evader at its estimated future location $\left(\hat{x}_{e}, \hat{y}_{e}\right)$. The proposed algorithm defines the angle difference $\delta_{p}$ and the predicted angle difference as follows,

$$
\begin{aligned}
& \delta_{p}=\tan ^{-1}\left(\frac{y_{e}-y_{p}}{x_{e}-x_{p}}\right)-\theta_{p}, \\
& \hat{\delta}_{p}=\tan ^{-1}\left(\frac{\hat{y}_{e}-y_{p}}{\hat{x}_{e}-x_{p}}\right)-\theta_{p} .
\end{aligned}
$$

In a pursuit-evasion game, when a fuzzy reinforcement learning algorithm tunes the FLC of the pursuer at the time step $t$ based on the real position of the evader defined at this time step, the pursuer at the time step $t+1$ will move towards the previous position of the evader defined by the pursuer at the time step $t$. This is because the FLC of the pursuer is tuned so that the angle difference $\delta_{p}$ defined by Eq. (5.9) at the time step $t$ is minimized. Thus, if the direction of the evader at the time step $t+1$ is similar to the direction of the evader at the time step $t$, the capture of the evader by the pursuer will be guaranteed if the pursuer moves in the 
same direction of the evader. In other words, if a faster pursuer chases a slower evader along a straight line, then the capture of the evader is a certainty. In some pursuit-evasion games, the evader may adopt an intelligent control strategy that takes advantage of its high maneuverability to avoid being captured. In such cases, the pursuer cannot easily capture the evader even if the pursuer is faster than the evader. To overcome this difficulty, the proposed algorithm tunes the parameters of the FLC based on the future position of the evader predicted by a Kalman filter. That is, the proposed algorithm predicts the future position of the evader based on a dead reckoning model and then tunes the FLC of the pursuer so that the pursuer moves directly to the expected position of the evader, where the capture will occur. In other words, the proposed algorithm tunes the FLC of the pursuer so that the predicted angle difference $\hat{\delta}_{p}$ is minimized. Moving directly to the predicted position of the evader will help the pursuer to eliminate or reduce the effect of:

1) the maneuverability of the evader, as the pursuer will predict the position of the evader and will directly move to that position, where the capture will occur.

2) the environmental changes, as the effect of these changes will be eliminated or reduced when the pursuer is tuned to move directly to the expected position of the evader instead of moving to the real position of the evader.

Fig. 5.2 shows the structure of the proposed KFFACL algorithm. The figure shows that a Kalman filter is being used by the proposed algorithm to estimate the future position of the evader which is then used to define the predicted state at the next time step, $\hat{\mathbf{s}}_{t+1}$. This predicted state $\hat{\mathbf{s}}_{t+1}$ is used as an input to the FLC of the pursuer to generate the control signal $u_{t}$ so that the pursuer can capture the evader at its estimated future location. Algorithm 7 illustrates the proposed KFFACL 


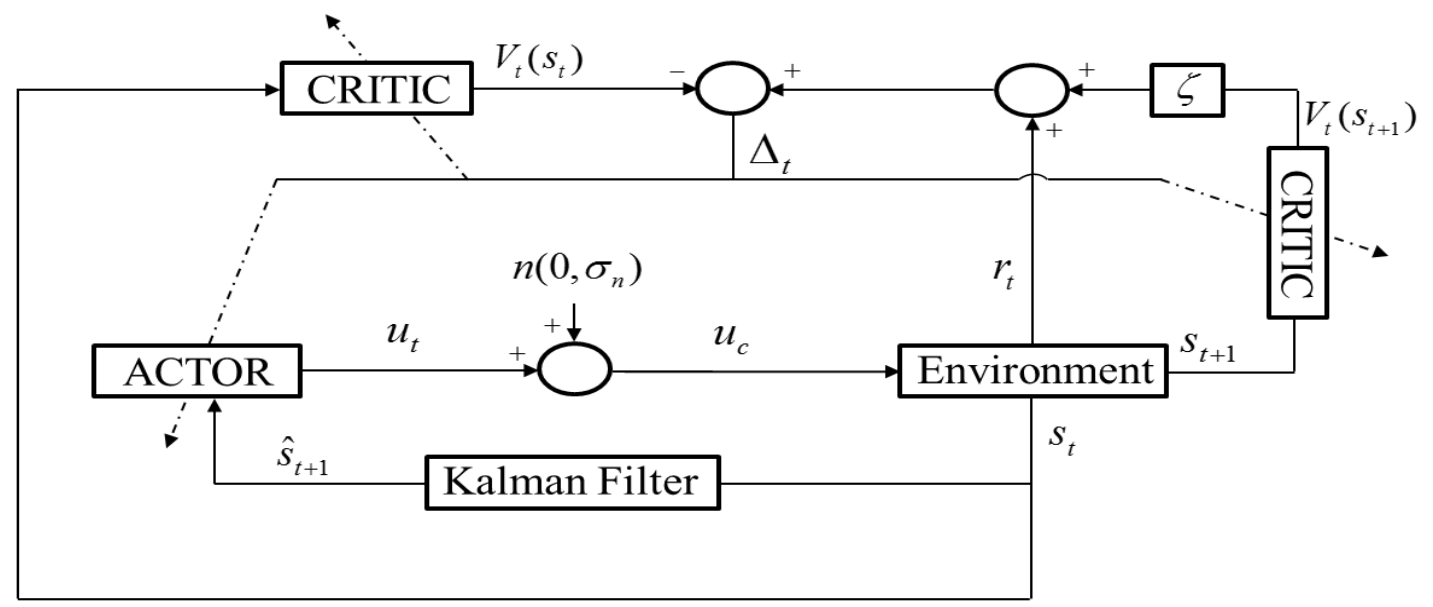

Figure 5.2: The proposed KFFACL algorithm.

algorithm.

\subsection{Simulation and Results}

We evaluate the proposed KFFACL algorithm, the RGFACL algorithm, the FACL algorithm and the QLFIS algorithm on different single pursuit-evasion games. These pursuit-evasion games have environments different from the training environment that the pursuer was trained on. That is, we train the pursuer in simulation. Then, the trained pursuer is used to capture the evader in these pursuit-evasion games, where the evaders in these games are different from the evader that was used in the training phase. In each pursuit-evasion game, the pursuer is faster than the evader, and the maneuverability of the evader is similar to or higher than the maneuverability of the pursuer. In addition, the evader follows an intelligent control strategy defined by Eq. (4.30) and Eq. (4.31). This intelligent strategy exploits the advantage of the maneuverability of the evader. 
Algorithm 7 The Kalman Filter Fuzzy Actor Critic Learning (KFFACL) algorithm.

(1) Initialize:

(a) All I/O parameters of the critics and the actor.

(b) All initial conditions of the algorithm and the Kalman filter.

(2) For each EPISODE do:

(3) Update the learning rates $\alpha$ and $\beta$ of the critics and the actor, respectively.

(4) Initialize the position of the pursuer at $\left(x_{p}, y_{p}\right)=0$ and the position of the evader randomly at $\left(x_{e}, y_{e}\right)$.

(5) Calculate $\delta_{p}$ from Eq. (5.9) and then calculate the initial state, $\mathbf{s}_{t}=(\delta, \dot{\delta})$.

(6) For each ITERATION do:

(7) Predict the future position of evader $\left(\hat{x}_{e}, \hat{y}_{e}\right)$ by using the Kalman filter.

(8) Calculate $\hat{\delta}_{p}$ from Eq. (5.10) and then calculate the predicted state, $\hat{\mathbf{s}}_{t+1}=(\hat{\delta}, \dot{\hat{\delta}})$.

(9) Calculate the output of the FLC, $u_{t}$, at the predicted state $\hat{\mathbf{s}}_{t+1}$ by using Eq.

(4.14) and then calculate the output $u_{c}=u_{t}+n\left(0, \sigma_{n}\right)$.

(10) Calculate the output of the critic, $V_{t}\left(\mathbf{s}_{t}\right)$, at the state $\mathbf{s}_{t}$ by using Eq. (4.14).

(11) Observe the next state $\mathbf{s}_{t+1}$ and the reward $r_{t}$.

(12) Calculate the output of the second critic, $V_{t}\left(\mathbf{s}_{t+1}\right)$, at the next state $\mathbf{s}_{t+1}$ by using Eq. (4.14).

(13) Calculate the temporal difference error, $\Delta_{t}$, by using Eq. (4.34).

(14) Update the input and the output parameters of the critics by using Eq. (4.38).

(15) Update the input and the output parameters of the actor by using Eq. (4.82).

(16) Set $\mathbf{s}_{t} \leftarrow \mathbf{s}_{t+1}$.

(17) Check Termination Condition.

(18) end for loop (ITERATION).

(19) end for loop (EPISODE).

\subsubsection{Simulation Setup}

The state $\mathbf{s}_{t}$ of the pursuer is defined by the two input variables, the pursuer's angle

difference $\delta_{p}$ and its derivative $\dot{\delta}_{p}$. On the other hand, the predicted state $\hat{\mathbf{s}}_{t}$ is defined by the pursuer's predicted angle difference $\hat{\delta}_{p}$ and its derivative $\dot{\hat{\delta}}_{p}$. Three Gaussian membership functions (MFs) are used to define the fuzzy sets of each input. The parameters of the pursuer are set as follows, $V_{p}=2.0 \mathrm{~m} / \mathrm{s}, L_{p}=0.3 \mathrm{~m}$ and $u_{p} \in[-0.5,0.5]$. The pursuer starts its motion from the position $\left(x_{p}, y_{p}\right)=(0,0)$ with an initial orientation $\theta_{p}=0$. The parameters of the evader in the training environment are set as follows, 
The training environment: $V_{e}=0.70 \mathrm{~m} / \mathrm{s}, L_{e}=0.3 \mathrm{~m}$ and $u_{e} \in[-1.00,1.00]$.

The evader in the training phase starts its motion from a random position at each episode. The sampling time is defined as $T=0.05 \mathrm{~s}$, whereas the capture radius is defined as $d_{c}=0.1 \mathrm{~m}$. The learning rates of the proposed KFFACL algorithm, the RGFACL algorithm, the FACL algorithm and the QLFIS algorithm are chosen as those presented in [100]. We set the process and the observation noise covariance matrices, $Q_{k f}$ and $R_{k f}$, based on trial and error as follows,

$$
Q_{k f}=\sigma_{q}^{2}\left[\begin{array}{cccc}
\frac{T^{4}}{4} & \frac{T^{3}}{2} & 0 & 0 \\
\frac{T^{3}}{2} & T^{2} & 0 & 0 \\
0 & 0 & \frac{T^{4}}{4} & \frac{T^{3}}{2} \\
0 & 0 & \frac{T^{3}}{2} & T^{2}
\end{array}\right], \quad \text { and } R_{k f}=\sigma_{r}^{2}\left[\begin{array}{cc}
1 & 0 \\
0 & 1
\end{array}\right] .
$$

We set $\sigma_{q}=100$ and $\sigma_{r}=0.001$.

We first train the pursuer with the training environment mentioned above so that the pursuer can learn its strategy. The number of episodes used in the training phase is set to 200, whereas the number of steps (in each episode) is set to 3000 . The evader follows the control strategy defined by Eq. (4.30) and Eq. (4.31) with the advantage of using its higher maneuverability. When the pursuer learns its strategy, we then apply the trained pursuer to different pursuit-evasion games that have environments different from the environment that was used in the training phase.

We use different types of pursuit-evasion games to evaluate the proposed KFFACL algorithm, the RGFACL algorithm, the FACL algorithm and the QLFIS algorithm. 
The parameters of these pursuit-evasion games are chosen without violating the assumptions we made about the studied pursuit-evasion games (the pursuer is faster than the evader and the maneuverability of the evader is similar to or higher than the maneuverability of the pursuer). Some of these games are chosen so that the maneuverability of the evader of each game is close to the maneuverability of the pursuer. The speed of the evader in these games varies from half to a very close value of the speed of the pursuer. The other games, on the other hand, are chosen so that the maneuverability of the evader in these games is twice or more of the maneuverability of the pursuer. The speed of the evader in these games is almost half of the speed of the pursuer. It is important to mention here that we do not use evaders with high speed and high maneuverability at the same time as capturing of such evaders may not be guaranteed in this case. Hence, we evaluate the proposed KFFACL algorithm and the other algorithms with five different pursuit-evasion games, where the parameters of the evader in each game are set as follows,

Game 1: $V_{e}=1.50 \mathrm{~m} / \mathrm{s}, L_{e}=0.3 \mathrm{~m}$ and $u_{e} \in[-0.60,0.60]$,

Game 2: $V_{e}=1.70 \mathrm{~m} / \mathrm{s}, L_{e}=0.3 \mathrm{~m}$ and $u_{e} \in[-0.50,0.50]$,

Game 3: $V_{e}=1.00 \mathrm{~m} / \mathrm{s}, L_{e}=0.3 \mathrm{~m}$ and $u_{e} \in[-1.00,1.00]$,

Game 4: $V_{e}=1.25 \mathrm{~m} / \mathrm{s}, L_{e}=0.3 \mathrm{~m}$ and $u_{e} \in[-1.00,1.00]$,

Game 5: $V_{e}=1.25 \mathrm{~m} / \mathrm{s}, L_{e}=0.3 \mathrm{~m}$ and $u_{e} \in[-1.25,1.25]$.

\subsubsection{Results and Analysis}

Table 5.1 to Table 5.5 show the time that the pursuer trained by each algorithm takes to capture the evader when the trained pursuer is used to capture the evader in different pursuit-evasion games, Game 1 to Game 5. The tables show the capture time of the evader when the evader, in each game, starts its motion from different 
positions. Table 5.1 and Table 5.2 show that the pursuer trained by each algorithm succeeds to capture the evader. This is because the maneuverability of the evader in Game 1 and Game 2 is similar or very close to the maneuverability of the pursuer. Hence, it will not be difficult for the pursuer trained by each algorithm to capture the evader. On the other hand, Table 5.3 to Table 5.5 show that the trained pursuer fails to capture the evader at some pursuit-evasion games when the pursuer was trained by the FACL, the QLFIS and the RGFACL algorithms. This is because the evader in these games (Game 3 to Game 5) is more maneuverable than the pursuer (i.e. the capture of the evader will not be an easy task for the pursuer). The notation "N.C" in the tables indicates to no capture occurred (i.e. the pursuer fails to capture the evader). The tables also show that only the pursuer that was trained by the proposed KFFACL algorithm succeeds to capture the evader in these games. This shows that the proposed KFFACL algorithm outperforms the FACL, the QLFIS and the RGFACL algorithms in terms of the ability of the pursuer to capture the evader and the capture time in such pursuit-evasion games.

Table 5.1: The time (in seconds) that the pursuer trained by each algorithm takes to capture the evader of Game 1. The evader starts motion at different positions.

\begin{tabular}{|l||c|c|c|c|}
\hline Algorithm & $(-5,9)$ & $(-10,-6)$ & $(7,4)$ & $(5,-10)$ \\
\hline \hline FACL & 29.40 & 30.90 & 27.20 & 25.05 \\
\hline QLFIS & 28.55 & 30.85 & 27.05 & 24.65 \\
\hline RGFACL & 27.30 & 29.25 & 25.35 & 23.15 \\
\hline The proposed KFFACL & 24.65 & 27.85 & 24.90 & 22.05 \\
\hline
\end{tabular}


Table 5.2: The time (in seconds) that the pursuer trained by each algorithm takes to capture the evader of Game 2. The evader starts motion at different positions.

\begin{tabular}{|l||c|c|c|c|}
\hline Algorithm Evader & $(-5,9)$ & $(-10,-6)$ & $(7,4)$ & $(5,-10)$ \\
\hline \hline FACL & 48.65 & 49.10 & 46.80 & 42.25 \\
\hline QLFIS & 44.10 & 47.35 & 43.20 & 40.40 \\
\hline RGFACL & 42.65 & 46.50 & 40.00 & 36.15 \\
\hline The proposed KFFACL & 38.25 & 40.80 & 36.30 & 32.45 \\
\hline
\end{tabular}

Table 5.3: The time (in seconds) that the pursuer trained by each algorithm takes to capture the evader of Game 3. The evader starts motion at different positions.

\begin{tabular}{|l||c|c|c|c|}
\hline Algorithm Evader & $(-5,9)$ & $(-10,-6)$ & $(7,4)$ & $(5,-10)$ \\
\hline \hline FACL & N.C & N.C & N.C & N.C \\
\hline QLFIS & N.C & N.C & N.C & N.C \\
\hline RGFACL & 15.75 & 18.25 & 12.60 & 15.05 \\
\hline The proposed KFFACL & 12.00 & 14.45 & 10.75 & 9.50 \\
\hline
\end{tabular}

\subsection{Summary and Discussion}

In a pursuit-evasion game, the pursuer trained by any learning algorithm can usually capture the evader when the environment of the pursuit-evasion game is similar to the training environment that the pursuer was trained on. However, the trained pursuer may not be able to capture the evader if the trained pursuer is applied to a pursuitevasion game that has an environment different from the training environment. In this chapter, we propose a new fuzzy reinforcement learning algorithm for pursuit-evasion games so that the pursuer trained by the proposed algorithm will be able to capture the evader even when the environment of the game is different from the training environment. The proposed algorithm is called the Kalman filter fuzzy actor critic learning (KFFACL) algorithm. The mechanism of the proposed KFFACL algorithm 
Table 5.4: The time (in seconds) that the pursuer trained by each algorithm takes to capture the evader of Game 4. The evader starts motion at different positions.

\begin{tabular}{|l||c|c|c|c|}
\hline Algorithm Evader & $(-5,9)$ & $(-10,-6)$ & $(7,4)$ & $(5,-10)$ \\
\hline \hline FACL & N.C & N.C & N.C & N.C \\
\hline QLFIS & N.C & N.C & N.C & N.C \\
\hline RGFACL & N.C & N.C & N.C & N.C \\
\hline The proposed KFFACL & 18.25 & 16.75 & 16.60 & 12.55 \\
\hline
\end{tabular}

Table 5.5: The time (in seconds) that the pursuer trained by each algorithm takes to capture the evader of Game 5. The evader starts motion at different positions.

\begin{tabular}{|l||c|c|c|c|}
\hline Algorithm Evader & $(-5,9)$ & $(-10,-6)$ & $(7,4)$ & $(5,-10)$ \\
\hline \hline FACL & N.C & N.C & N.C & N.C \\
\hline QLFIS & N.C & N.C & N.C & N.C \\
\hline RGFACL & N.C & N.C & N.C & N.C \\
\hline The proposed KFFACL & 16.00 & 16.90 & 16.70 & 12.55 \\
\hline
\end{tabular}

is to tune the parameters of the FLC of the pursuer based on the future position of the evader predicted by a Kalman filter. That is, the proposed KFFACL algorithm tunes the FLC of the pursuer so that the pursuer moves directly to the expected position of the evader, where the capture will occur. Moving directly to the predicted position of the evader will help the pursuer to eliminate or reduce the effect of the maneuverability of the evader and the environmental changes that may occur. We evaluate the FACL, the QLFIS, the RGFACL and the proposed KFFACL algorithms to see how quickly each algorithm will adapt to the new and different environment and be able to capture the different evader. Different single pursuit-evasion games are used in our evaluation. Each game of these pursuit-evasion games has an environment different from the training environment the pursuer was trained on. Simulation results 
show that the proposed KFFACL algorithm outperforms the fuzzy reinforcement learning algorithms (FACL, QLFIS and RGFACL) in terms of the ability of the pursuer to capture the evader and the capture time in such pursuit-evasion games. 


\section{Chapter 6}

\section{Decentralized Learning in Multi-Pursuer}

Pursuit-Evasion Differential Games with a Single Evader Having the Same Speed as

\section{the Pursuers}

\subsection{Introduction}

Multi-player pursuit-evasion games have received more attention in recent years $[75,77,78]$. One of the approaches used in the literature to optimize the performance of pursuers in multi-player pursuit-evasion games is the hierarchical decomposition approach. This approach decomposes the multi-player pursuit-evasion game into small games between pursuers and evaders. That is, one-pursuer oneevader games, or multi-pursuer single-superior-evader games [72,77]. A superior evader is defined as an evader that has a speed which is similar to or higher than the speed of all pursuers. Cooperation among pursuers is another approach that is used in the literature to optimize the performance of the pursuers in multi-player pursuit-evasion games and to make them act as a whole to perform their mission task. One of the popular techniques used to facilitate cooperation among pursuers 
in a multi-player pursuit-evasion game is formation control [72], which shapes the relative position and the orientation of pursuers.

A number of articles [54,61, 63-65, 100, 101] investigated pursuit-evasion games with slow evaders, where the capture of the evader is always possible. However, in real-world applications, evaders may run with speed similar to or higher than the speed of pursuers. In such cases, the capture of the faster (superior) evader may require more than one pursuer. This has led researchers to investigate multi-pursuer single-superior-evader pursuit-evasion games and propose a number of different techniques so that one or some of the pursuers can capture the superior evader $[72-75,78-80,82,83]$. These techniques use different mechanisms such as hierarchical decomposition approaches and formation control approaches to tune the pursuers. However, all these techniques are deterministic approaches and involve no learning in the tuning mechanisms of the pursuers. In real-world applications, pursuers may need to adapt to environment changes.

The only article that considers the use of learning in multi-player pursuit-evasion differential games with superior evaders is proposed in [81]. The authors of this article propose their algorithm based on three basic behaviors of each pursuer (namely Move-to-goal, Avoid-obstacle and Hunting), and learning is only involved in one behavior (Hunting). The authors of this article structure the learning part of their algorithm based on the Q-learning algorithm. The Q-learning algorithm requires discrete state and action spaces, as such the authors used a state-space reduction mechanism to deal with the continuous spaces. However, in many real-world applications, a priori discretization of the action space may not be useful [84]. In addition, using a coarse discretization of the state or action space may lead to a poor performance [85-87]. 
In this chapter, we consider multi-pursuer single-superior-evader pursuit-evasion differential games, where the evader and the pursuers in these games have similar speeds. We use some of the formation control ideas presented in the literature from the learning point of view. Our objective is to make the pursuers in a multi-pursuer single-superior-evader pursuit-evasion differential game learn their strategies so that one or some of the learning pursuers can capture the superior evader. This work is the first piece of work that presents a decentralized learning algorithm to capture a superior evader in a pursuit-evasion differential game without using any type of discretization for the state or action spaces. The proposed work is published in [138].

\subsection{Problem Definition}

In this chapter, we consider a pursuit-evasion game with $N$ pursuers and one superior evader $E$. Each pursuer $P_{o},(o=1, \ldots, N)$, has a constant speed $V_{p_{o}}$ that is the same as the other pursuers' speeds. The evader also has a constant speed $V_{e}$, where $V_{e}=V_{p_{o}}$. In the next chapter, we will consider the case that $V_{e} \geq V_{p_{o}}$. The positions of the pursuers and the evader are $\left(x_{p_{o}}(t), y_{p_{o}}(t)\right)$ and $\left(x_{e}(t), y_{e}(t)\right)$, respectively. We assume that each pursuer $P_{o}$ knows the position of the evader at every time step $t$. We also assume that the constant speed of the evader is known to each pursuer $P_{o}$. The capture of the superior evader occurs if the distance between the pursuer $P_{o}$ and the evader $E$ is less than or equal to a small specific amount $d_{c},\left\|\left(x_{p_{o}}, y_{p_{o}}\right)-\left(x_{e}, y_{e}\right)\right\| \leq d_{c}$.

Fig. (6.1) shows that the pursuer $P_{o}$ succeeds to capture the evader $E$ at the point I. When the pursuer captures the evader as shown in Fig. (6.1), then by the triangle property, we have $[72,73,75,76,78]$, 


$$
\frac{\sin \alpha_{o}}{\sin \beta_{o}}=\frac{V_{e}}{V_{p_{o}}}
$$

where the angle $\beta_{o}$ is the angle difference between the direction of the evader and the direction of the evader's line of sight (LOS) to the pursuer $P_{o}$, and the angle $\alpha_{o}$ is the angle difference between the direction of the pursuer $P_{o}$ and the direction of the pursuer's LOS to the evader $E$.

When the evader moves into a region where the pursuer $P_{o}$ can select an angle $\alpha_{o}$ that satisfies the condition of Eq. (6.1), that region is called the capture region of the pursuer $P_{o}$. On the other hand, when the evader moves into a region where the pursuer $P_{o}$ cannot select an angle $\alpha_{o}$ that satisfies the condition of Eq. (6.1), that region is called the escape region of the pursuer $P_{o}$.

In this work, we assume that $V_{p_{o}}=V_{e}$. Thus, the capture condition of Eq. (6.1) can be rewritten as follows,

$$
\sin \alpha_{o}=\sin \beta_{o} .
$$

Thus,

$$
\alpha_{o}=\beta_{o} .
$$

From the capture condition of Eq. (6.3) and Fig. (6.1), the following inequality is valid if the evader moves into the capture region of the pursuer $P_{o}$,

$$
\alpha_{o}+\beta_{o}<\pi \text {. }
$$

Thus, from Eq. (6.3) and Eq. (6.4), we have, 


$$
\beta_{o} \in\left[0, \beta_{o}^{*}\right] \quad \text { and } \quad \beta_{o}^{*}<\pi / 2 \text {. }
$$

We define the angle $\beta_{o}^{*}$ as the maximum angle that if the evader exceeds, the evader will move into the escape region of the pursuer $P_{o}$. Hence, as long as the evader's angle $\beta_{o}$ is not greater than $\beta_{o}^{*}$, the capture of the superior evader by the pursuer $P_{o}$ will be guaranteed if the pursuer is well tuned. Fig. (6.1) shows that the capture region of the pursuer $P_{o}$ can be represented by the angle $2 \beta_{o}$. In other words, the pursuer $P_{o}$ can capture the superior evader $E$ if the evader moves with an angle $\beta_{o}$ from the evader's LOS to the pursuer in the direction of either the boundary line $E I$ or the boundary line $E \bar{I}$. Hence, from Eq. (6.5), the capture region of the pursuer $P_{o}$ can be represented by the wedge $2 \beta_{o}^{*}$. From Eq. (6.5), if the evader moves with an angle $\beta_{o}$ such that $\beta_{o}<\pi / 2$, we can say that the evader moves into the capture region of the pursuer $P_{o}$. On the other hand, if the evader moves with an angle $\beta_{o}$ such that $\beta_{o} \geq \pi / 2\left(\right.$ or $\beta_{o}>\beta_{o}^{*}$ ), we can say that the evader moves into the escape region of the pursuer $P_{o}$.

From Eq. (6.3) and Eq. (6.5), the pursuer can capture the superior evader if the following condition is satisfied,

$$
\alpha_{o}=\beta_{o} \quad \text { and } \quad \beta_{o}<\pi / 2 \text {. }
$$

To guarantee the capture of the superior evader in a multi-pursuer single-superiorevader pursuit-evasion game with $V_{e}=V_{p_{o}}$, the pursuers have to surround the evader during the entire game. That is, the pursuers have to be distributed around the superior evader to construct a polygon whose vertices are the pursuers' positions $[72,74,78]$. The capture regions (wedges) of each two adjacent pursuers have to intersect or at least border each other so that the superior evader can be 


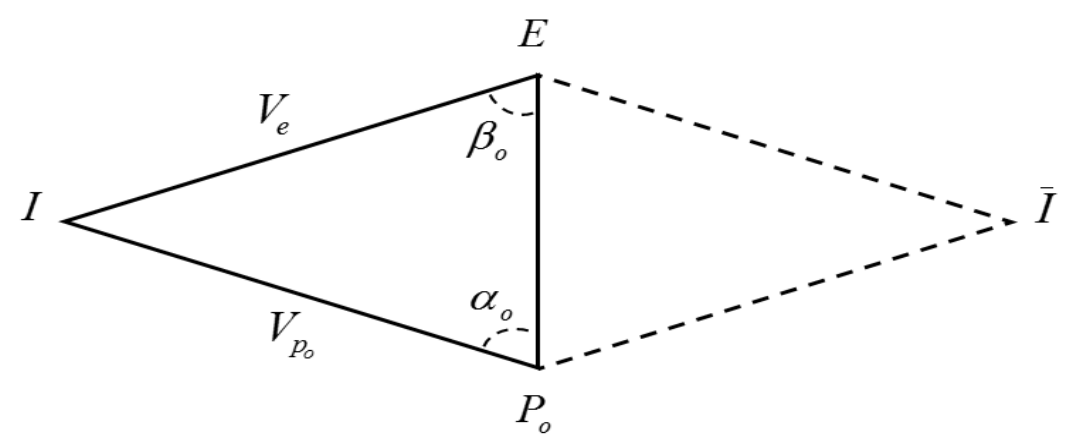

Figure 6.1: Geometric illustration of capture [72].

captured by one or some of the pursuers. Since the wedge that the pursuer $P_{o}$ can cover is $2 \beta_{o}^{*}$ and $\beta_{o}^{*}<\pi / 2$, at least three pursuers will be needed to cover the $2 \pi$ degrees around the superior evader. If the pursuers succeed to surround the evader all time and the wedges of each two adjacent pursuers intersect or at least border each other, there will be at least one pursuer that can satisfy the capture condition of Eq. (6.6) and then be able to capture the superior evader.

\subsection{The Proposed Algorithm}

In this work, we propose a decentralized learning algorithm to capture a superior evader of a differential pursuit-evasion game. This is the first time that anyone has shown that they can develop a system of learning to capture a superior evader in a differential pursuit-evasion game. There has been some work done in the literature to capture superior evaders $[72-75,78-80]$. However, these techniques are deterministic approaches as no learning is involved in these techniques. In real world applications, pursuers may need to adapt to environment changes. Our objective in this work is to make the pursuers in a multi-pursuer single-superior-evader pursuit-evasion differential game learn their strategies so that one or some of the learning pursuers 
can capture the superior evader. Different fuzzy learning algorithms that can be applied to pursuit-evasion differential games are proposed in the literature [99-101]. In this chapter, the proposed algorithm uses the RGFACL algorithm to tune the parameters of the FLC of each learning pursuer $P_{o}$ as the RGFACL algorithm is shown in [101] to be more robust and has a quicker convergence speed than the other (FACL and QLFIS) algorithms. The proposed algorithm is a decentralized algorithm as each learning pursuer $P_{o}$ has its own learning algorithm and no communication among pursuers is required. The only information the proposed learning algorithm of the pursuer $P_{o}$ requires is the position of the superior evader. The proposed algorithm uses a formation control approach as a mechanism to construct the reward function of the learning pursuer $P_{o}$. We mean by the formation control that how to shape the positions and the orientations of the pursuers around the superior evader. The formation control mechanism used by the proposed algorithm makes the learning pursuers surround the evader and construct a polygon whose vertices are the pursuers' positions. This will guarantee that the learning pursuers are distributed around the superior evader in order to avoid collision between pursuers. In addition, the formation control mechanism guarantees that the capture regions of each two adjacent pursuers overlap or at least border each other $[72-75,78]$ so that the capture equation of Eq. (6.6) is satisfied and the capture of the superior evader will be guaranteed.

\subsubsection{The Action $u_{p_{o}}$ of Pursuer $P_{o}$}

Fig (6.2-a) shows the pursuer $P_{o}$ and the evader $E$. We use a Kalman filter to estimate the future position of the evader $\hat{E}$ at the next time step. Fig (6.2-b) shows the pursuer $P_{o}$ and the evader at its real and estimated future positions. We define the angle $\beta_{o}$ as the absolute value of the angle difference between the direction of the

evader (the direction of the vector $\overrightarrow{E E}$ ) and the direction of the evader's LOS to the 
pursuer $P_{o}$ (the direction of the vector $\left.\overrightarrow{E P_{o}}\right)$. That is,

$$
\beta_{o}=\left|\bar{\beta}_{o}\right|
$$

where,

$$
\bar{\beta}_{o}=\angle \overrightarrow{E E}-\angle \overrightarrow{E P_{o}} \quad \text { and } \quad-\pi \leq \bar{\beta}_{o} \leq \pi
$$

Fig (6.2-c) shows the angle $\alpha_{o}$ that the pursuer $P_{o}$ should select in order to satisfy the capture equation defined in Eq. (6.6). That is, the angle that describes the direction $\theta_{\alpha_{o}}=\angle \overrightarrow{P_{o} I}$ that the pursuer $P_{o}$ should take in order to capture the evader at the future capture point $I$. To find the direction $\theta_{\alpha_{o}}$, we first define the vector $\overrightarrow{P_{o} I}$; the vector $\overrightarrow{P_{o} I}$ has the same length as the vector $\overrightarrow{P_{o} E}$ and the same direction as the vector $\overrightarrow{P_{o} I}$. We use the angle $\alpha_{o}$ and the vector $\overrightarrow{P_{o} E}$ to define the vector $\overrightarrow{P_{o}}$, as follows,

$$
\overrightarrow{P_{o} I}=R\left(\bar{\alpha}_{o}\right) \times \overrightarrow{P_{o} E}
$$

where $R\left(\bar{\alpha}_{o}\right)$ is a rotation matrix that is used to perform rotation in Euclidean space and it is defined as follows,

$$
R\left(\bar{\alpha}_{o}\right)=\left[\begin{array}{cc}
\cos \left(\bar{\alpha}_{o}\right) & -\sin \left(\bar{\alpha}_{o}\right) \\
\sin \left(\bar{\alpha}_{o}\right) & \cos \left(\bar{\alpha}_{o}\right)
\end{array}\right],
$$

and,

$$
\bar{\alpha}_{o}=\left\{\begin{array}{rrr}
-\alpha_{o} & \text { if } & \bar{\beta}_{o}>0, \\
\alpha_{o} & & \text { otherwise. }
\end{array}\right.
$$

The direction of rotation will be counter-clockwise if the angle $\bar{\alpha}_{o}$ is positive. On the 


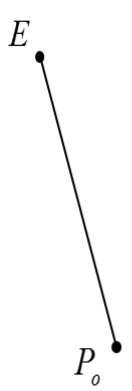

(a)

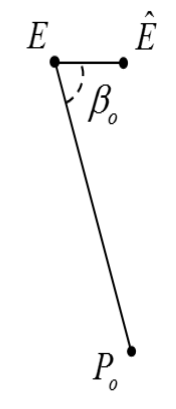

(b)

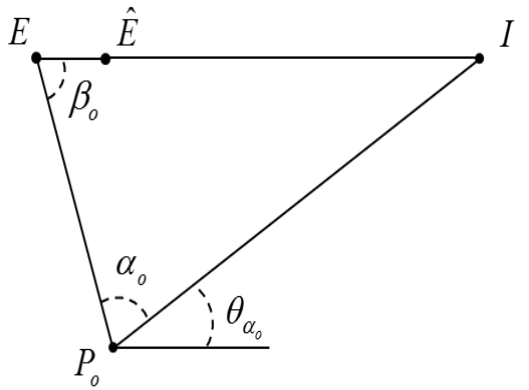

(c)

Figure 6.2: Geometric illustration of defining the action for the pursuer $P_{o}$.

other hand, the direction of rotation will be clockwise if the angle $\bar{\alpha}_{o}$ is negative.

Let the vector $\overrightarrow{P_{o} I}$ be defined as $\overrightarrow{P_{o} I}=\left(x_{d i r}, y_{d i r}\right)$. Thus, the direction $\theta_{\alpha_{o}}$, the direction of the vector $\overrightarrow{P_{o} I}$, is defined as follows,

$$
\theta_{\alpha_{o}}=\angle \overrightarrow{P_{o}}=\tan ^{-1}\left(\frac{y_{d i r}}{x_{d i r}}\right)
$$

Hence, the action $u_{p_{o}}$ selected by the pursuer $P_{o}$ at each sampling period is defined as follows,

$$
u_{p_{o}}=\theta_{\alpha_{o}}-\theta_{p_{o}}
$$

where $\theta_{p_{o}}$ is the direction (orientation) of the pursuer $P_{o}$.

\subsubsection{The Reward Function of Pursuer $P_{o}$}

In this work, we set the rewards of each learning pursuer based on a formation control mechanism so that one or some of the learning pursuers can capture the superior evader. The formation control mechanism used in this work is similar to the formation control mechanisms presented in $[72-75,78]$. In this formation control 
mechanism, the pursuer $P_{o}$ moves in a direction such that Eq. (6.6) is satisfied when the superior evader moves into the capture region of the pursuer $P_{o}$, and moves in parallel with the superior evader when the superior evader moves into the escape region of the pursuer $P_{o}$. This will guarantee that the learning pursuers are distributed around the superior evader so that collision between pursuers is avoided [72]. This will also guarantee that the capture regions of each two adjacent pursuers overlap or at least border each other so that the capture of the superior evader will be guaranteed by one or some of the learning pursuers $[72-75,78]$. The speed of the pursuer $P_{o}$ is defined by $V_{p_{o}}$, and the speed of the evader $E$ is defined by $V_{e}$. We assume that the speed of the evader is known to each pursuer. At each sampling period, the inputs to the FLC of the pursuer $P_{o}$ are the angle $\beta_{o}$ and its derivative $\dot{\beta}_{o}$. Based on its inputs, the FLC of the pursuer $P_{o}$ selects an angle $\alpha_{o}$ which is then used to calculate the action $u_{p_{o}}$ as in Eq. (6.13).

The pursuer $P_{o}$ will be rewarded based on the region the evader moves into; the capture region of the pursuer $P_{o}$ (when $\beta_{o}<\pi / 2$ ), and the escape region of the pursuer $P_{o}$ (when $\beta_{o} \geq \pi / 2$ ). We will first describe how to reward the pursuer $P_{o}$ when the evader moves into the capture region of the pursuer $P_{o}$. i.e. the evader moves with an angle $\beta_{o}$ such that $\beta_{o}<\pi / 2$. The reward function of the pursuer $P_{o}$, in this case, is constructed based on the triangle property defined in Eq. (6.1). That is, we use the capture equation defined in Eq. (6.6) as a mechanism to reward the learning pursuer $P_{o}$ at each sampling period. If the angle $\alpha_{o}$ selected by the pursuer $P_{o}$ satisfies the triangle property (capture equation) of Eq. (6.6), the pursuer $P_{o}$ will be rewarded with a positive payoff ( +1 for example). As illustrated in Eq. (6.5), the pursuer $P_{o}$ will be able to capture the evader only if the angle $\beta_{o}$ such that $\beta_{o}<\pi / 2$. This is also shown in Fig. (6.3-a). Thus, if the angle $\beta_{o}$ such that $\beta_{o}<\pi / 2$ and the angle $\alpha_{o}$ selected by the pursuer $P_{o}$ does not satisfy the capture equation defined in 
Eq. (6.6), the pursuer $P_{o}$ should be punished with a negative payoff ( -1 for example). Hence, the reward function of the pursuer $P_{o}$ when the evader moves into the capture region of the pursuer $P_{o}$ is defined as follows,

$$
r_{o}=\left\{\begin{array}{ccc}
+1 & \text { if } & \alpha_{o} \in\left[\beta_{o}-\epsilon_{a}, \beta_{o}+\epsilon_{a}\right] \& \beta_{o}<\pi / 2, \\
-1 & \text { if } \quad \alpha_{o} \notin\left[\beta_{o}-\epsilon_{a}, \beta_{o}+\epsilon_{a}\right] \& \beta_{o}<\pi / 2,
\end{array}\right.
$$

where $\epsilon_{a}$ is a very small constant that defines the angle tolerance.

On the other hand, when the evader moves into the evasion region of the pursuer $P_{o}$, the pursuer $P_{o}$ will not be rewarded based on the capture equation defined in Eq. (6.6). In this case, the evader moves with an angle $\beta_{o}$ such that $\beta_{o} \geq \pi / 2$. Thus, the pursuer $P_{o}$ has to work with the other pursuers to surround (enclose) the evader. That is, the pursuer $P_{o}$ has to move in parallel with the evader as shown in Fig. (6.3-b). This keeps the pursuers distributed around the evader. This also keeps the capture region of the pursuer $P_{o}$ overlapping or at least bordering with the capture regions of the other adjacent pursuers $[72-75,78]$. Hence, if the angle $\beta_{o}$ is such that $\beta_{o} \geq \pi / 2$ and the angle $\alpha_{o}$ selected by the pursuer $P_{o}$ makes the pursuer $P_{o}$ move in parallel with the evader, the pursuer will be rewarded with a positive payoff $(+1$ for example); otherwise the pursuer $P_{o}$ will be punished by receiving a negative payoff $(-1$ for example). To make the pursuer $P_{o}$ move in parallel with the evader, the angle $\alpha_{o}$ selected by the pursuer $P_{o}$ has to be such that $\alpha_{o}+\beta_{o}=\pi$. Thus, the reward function of the pursuer $P_{o}$ when the evader moves into the evasion region of the pursuer $P_{o}$ is defined as follows, 


$$
r_{o}=\left\{\begin{array}{lll}
+1 & \text { if } \quad \alpha_{o}+\beta_{o} \in\left[\pi-\epsilon_{a}, \pi+\epsilon_{a}\right] \& \beta_{o} \geq \pi / 2, \\
-1 \quad \text { if } \quad & \alpha_{o}+\beta_{o} \notin\left[\pi-\epsilon_{a}, \pi+\epsilon_{a}\right] \& \beta_{o} \geq \pi / 2,
\end{array}\right.
$$

where $\epsilon_{a}$ is a very small constant that defines the angle tolerance.

Hence, from Eq. (6.14) and Eq. (6.15), the reward function of the pursuer $P_{o}$ when the evader moves into either the capture region or the evasion region of the pursuer $P_{o}$ is defined as follows,

$$
r_{o}=\left\{\begin{array}{rll}
1 & \text { if } \quad \alpha_{o} \in\left[\beta_{o}-\epsilon_{a}, \beta_{o}+\epsilon_{a}\right] \& \beta_{o}<\pi / 2 \\
1 & \text { if } \quad \alpha_{o}+\beta_{o} \in\left[\pi-\epsilon_{a}, \pi+\epsilon_{a}\right] \& \beta_{o} \geq \pi / 2 \\
-1 & \text { otherwise. }
\end{array}\right.
$$

As can be seen in Eq. (6.16), when the angle $\beta_{o}$ is such that $\beta_{o}<\pi / 2$, the priority of learning for the pursuer $P_{o}$ is to learn how to capture the evader. On the other hand, when the angle $\beta_{o}$ is such that $\beta_{o} \geq \pi / 2$, the priority of learning for the pursuer $P_{o}$ is learn how to to move in parallel with the evader. It is important to mention here that when the angle $\beta_{o}$ is such that $\beta_{o} \geq \pi / 2$, the pursuer $P_{o}$ will not be able to capture the evader as there will be no such angle $\alpha_{o}$ that can satisfy the capture equation defined in Eq. (6.6). Thus, Eq. (6.16) can be modified in a simpler form by taking the condition related to $\beta_{o}$, in the second line, out of the equation. That is,

$$
r_{o}=\left\{\begin{array}{rll}
1 & \text { if } & \alpha_{o} \in\left[\beta_{o}-\epsilon_{a}, \beta_{o}+\epsilon_{a}\right] \& \beta_{o}<\pi / 2, \\
0.1 \quad \text { if } & \alpha_{o}+\beta_{o} \in\left[\pi-\epsilon_{a}, \pi+\epsilon_{a}\right] \\
-1 & \text { otherwise. }
\end{array}\right.
$$




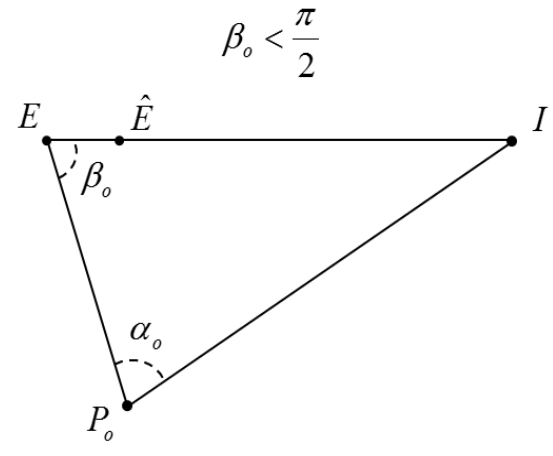

(a)

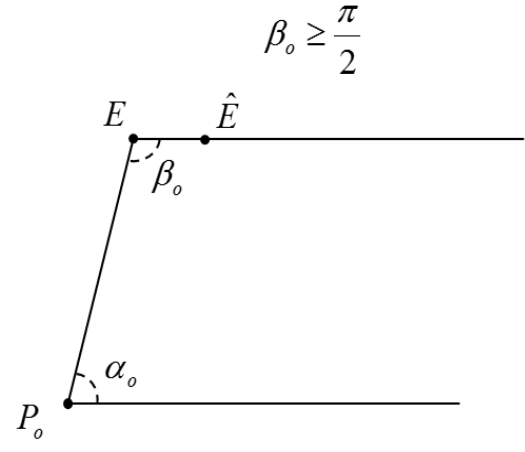

(b)

Figure 6.3: Geometric illustration of the reward function mechanism for the pursuer $P_{o}$.

Eq. (6.17) indicates that the first priority of learning for the pursuer $P_{o}$ is to learn how to capture the evader. When the capture of the evader is not possible, the pursuer $P_{o}$ will go to its second priority of learning (learning how to move in parallel with the evader) in order to avoid being punished with a negative payoff.

\subsection{Simulation and Results}

In this section, we evaluate the proposed algorithm on a multi-pursuer singlesuperior-evader pursuit-evasion differential game where the speed of the evader is similar to the speed of each pursuer. In this game, we use three pursuers (i.e. $o=1,2,3)$ and one superior evader. Each pursuer is learning its control strategy so that one or some of the learning pursuers can capture the superior evader. The evader is also learning its strategy so that it can reach a specific target before being captured by one or some of the pursuers. It is important to mention here that our objective in this simulation is not to set a complicated learning mechanism for the evader. We want only to evaluate our proposed decentralized algorithm and see 
whether or not the learning pursuers will succeed to capture a superior evader that changes its direction during the pursuit. Thus, we set a simple learning mechanism for the evader so that the priority of the evader is to learn how to reach its target. However, if the distance between the evader and the nearest pursuer is less than a predefined distance (tolerance distance, $d_{t o l}$ ), the priority of the evader becomes to learn how to escape from that pursuer.

As illustrated in the previous section, for each pursuer $P_{o}$, we define the angle $\beta_{o}$ as the absolute value of the angle difference between the direction of the evader and the direction of the evader's LOS to the pursuer $P_{o}$. In addition, we define the state $\mathbf{s}_{t}$ for the pursuer $P_{o}$ by the two input variables which are the angle $\beta_{o}$ and its derivative $\dot{\beta}_{o}$. Five Gaussian membership functions (MFs) are used to define the fuzzy sets of each input to the FISs of each pursuer $P_{o}$. On the other hand, the state $\mathbf{s}_{t}$ of the evader is defined by two input variables, $\delta_{e}$ and its derivative $\dot{\delta}_{e}$, where $\delta_{e}$ is the angle difference between the direction of the evader and the direction of its target. However, if the distance between the evader and the nearest pursuer is less than the tolerance distance $d_{t o l}, \delta_{e}$ is defined as the angle difference between the direction of the evader and the direction of the LOS of that nearest pursuer to the evader. We define the tolerance distance $d_{t o l}$ as $d_{t o l}=10 \mathrm{~m}$. Five Gaussian membership functions (MFs) are used to define the fuzzy sets of each input to the FISs of the evader. The parameters of the pursuers and the evader are set as follows, $V_{p_{o}}=V_{e}=1.0 \mathrm{~m} / \mathrm{s}$, $L_{p_{o}}=L_{e}=0.5 \mathrm{~m}$ and $u_{p_{o}}=u_{e} \in[-0.6,0.6]$.

The evader starts its motion from the position $\left(x_{e}, y_{e}\right)=(0,0)$ with an initial orientation $\theta_{e}=0$. The pursuer $P_{1}$ starts its motion from a random position at $d_{1} \angle \theta_{d_{1}} ; d_{1}$ is the initial distance between the pursuer $P_{1}$ and the evader $E$, and $\theta_{d_{1}}$ is the initial angle that describes the direction of the vector $\overrightarrow{E P_{1}}$. Thus, the 
random position of the pursuer $P_{1}$ is defined as $\left(x_{p_{1}}, y_{p_{1}}\right)=\left(d_{1} \cos \theta_{d_{1}}, d_{1} \sin \theta_{d_{1}}\right)$. The initial orientation of the pursuer $P_{1}$ is defined as $\theta_{p_{1}}=\tan ^{-1}\left(\frac{-y_{p_{1}}}{-x_{p_{1}}}\right)$. Similarly, the pursuers $P_{2}$ and $P_{3}$ start their motions from random positions but taking into account the distribution of the pursuers around the evader required by the formation control mechanism. Since we have three pursuers, we define the initial positions of the pursuers $P_{2}$ and $P_{3}$ as $\left(x_{p_{2}}, y_{p_{2}}\right)=\left(d_{2} \cos \left(\theta_{d_{1}}+2 \pi / 3\right), d_{2} \sin \left(\theta_{d_{1}}+2 \pi / 3\right)\right)$ and $\left(x_{p_{3}}, y_{p_{3}}\right)=\left(d_{3} \cos \left(\theta_{d_{1}}+4 \pi / 3\right), d_{3} \sin \left(\theta_{d_{1}}+4 \pi / 3\right)\right)$, respectively. The distances $d_{2}$ and $d_{3}$ are randomly selected. The initial orientations of the pursuers $P_{2}$ and $P_{3}$ are defined as $\theta_{p_{2}}=\tan ^{-1}\left(\frac{-y_{p_{2}}}{-x_{p_{2}}}\right)$ and $\theta_{p_{3}}=\tan ^{-1}\left(\frac{-y_{p_{3}}}{-x_{p_{3}}}\right)$, respectively. The parameters of the RGFACL learning algorithm are set as those parameters in [101]. The sampling time is defined as $T=0.05 \mathrm{~s}$, whereas the capture radius is defined as $d_{c}=0.3 \mathrm{~m}$. The number of episodes is set to 500, whereas the number of steps (in each episode) is set to 2000 .

Fig. (6.4) to Fig. (6.7) show the paths of the pursuers when each pursuer learns its control strategy by the proposed algorithm. Fig. (6.4) to Fig. (6.7) also show the path of the evader when the evader learns its control strategy by the RGFACL algorithm. The initial positions of the pursuers are set as $\left(x_{p_{1}}, y_{p_{1}}\right)=40 \angle 0.5$, $\left(x_{p_{2}}, y_{p_{2}}\right)=50 \angle(0.5+2 \pi / 3)$ and $\left(x_{p_{3}}, y_{p_{3}}\right)=30 \angle(0.5+4 \pi / 3)$. The evader starts its motion from the position $(0,0)$ and moves towards its target. In each figure, the evader has a specific target that the evader wants to reach before being captured by the pursuers. As shown in Fig. (6.4) to Fig. (6.7), the pursuers trained by the proposed algorithm enclose the superior evader and construct a polygon whose vertices are the pursuers' positions. The pursuers continue surrounding the superior evader and shrinking the enclosed polygon. When the distance between the evader and the nearest pursuer is less than the tolerance distance (which we define here as $\left.d_{t o l}=10 \mathrm{~m}\right)$, the evader tries to escape from that pursuer. Surrounding 


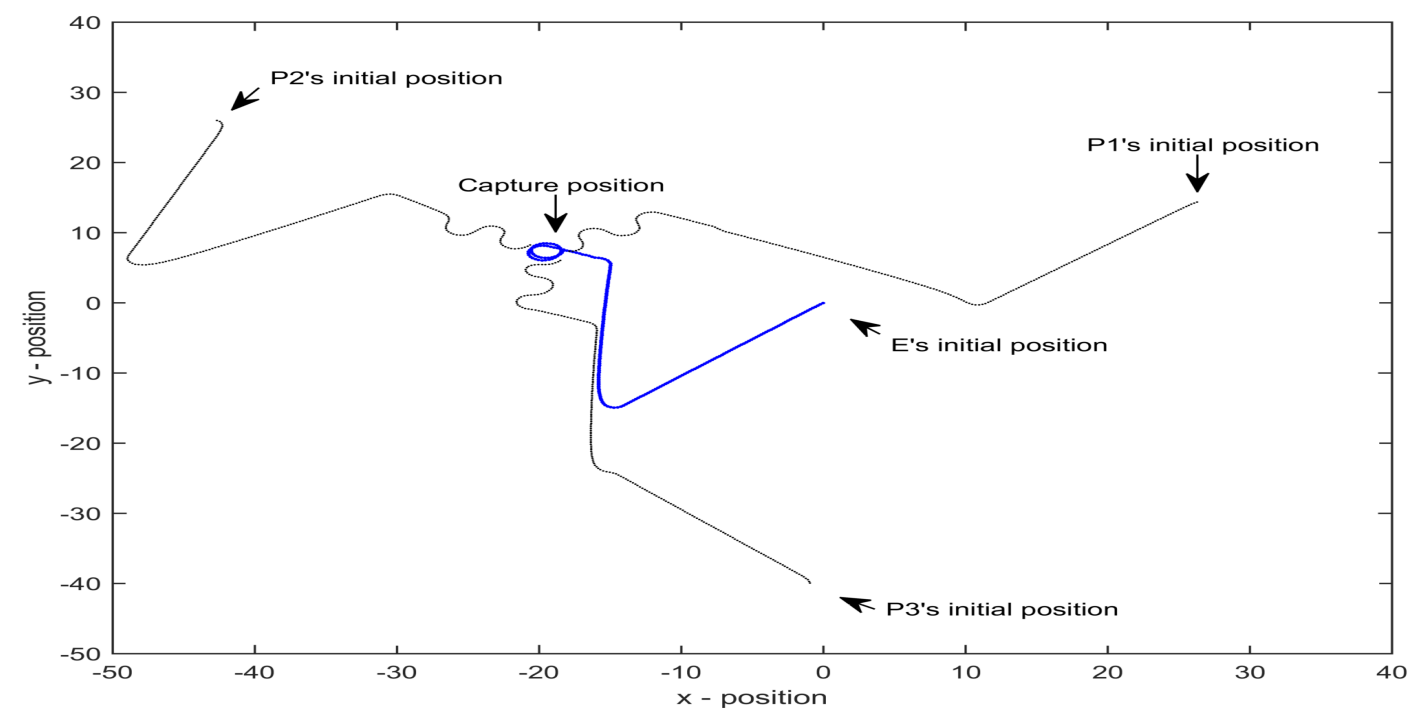

Figure 6.4: The paths of the pursuers (thin-line paths) when each pursuer $P_{o}$ learns its control strategy by the proposed algorithm; the path of the evader (thick-line path) when the evader learns its control strategy by the RGFACL algorithm. The target of the evader here is the position $(x, y)=(-500,-500)$.

the superior evader and shrinking the enclosed polygon by the pursuers will also make the capture region of each pursuer overlap or at least border the capture regions of its adjacent pursuers. Because of this mechanism, the evader is always enclosed by the pursuers until it is eventually captured by one or some of the pursuers.

\subsection{Summary and Discussion}

In this chapter, we propose a new decentralized fuzzy reinforcement learning algorithm that tunes the pursuers in a multi-pursuer single-superior-evader pursuit-evasion differential game so that the superior evader is captured by one or some of the pursuers. We mean by the "superior evader" that the evader that has a speed that is similar to the speed of each pursuer in the game. The proposed algorithm uses the 


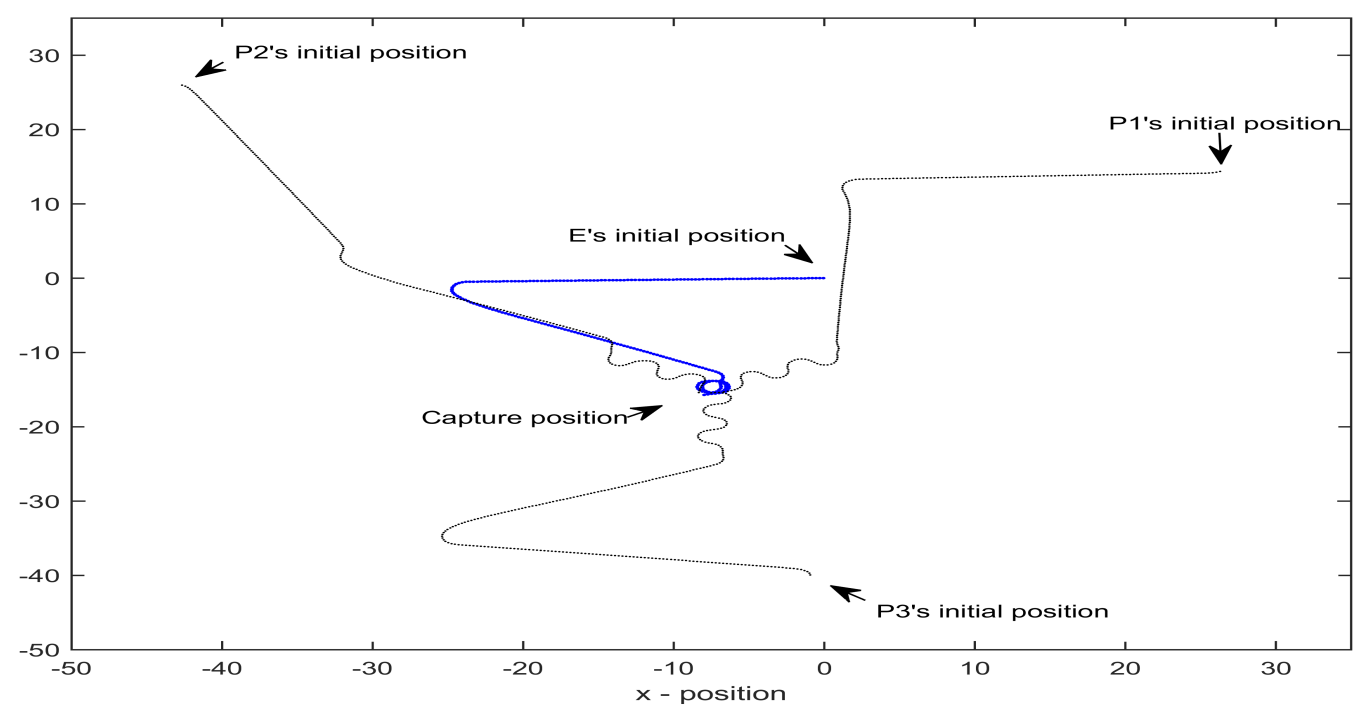

Figure 6.5: The paths of the pursuers (thin-line paths) when each pursuer $P_{o}$ learns its control strategy by the proposed algorithm; the path of the evader (thick-line path) when the evader learns its control strategy by the RGFACL algorithm. The target of the evader here is the position $(x, y)=(-500,0)$.

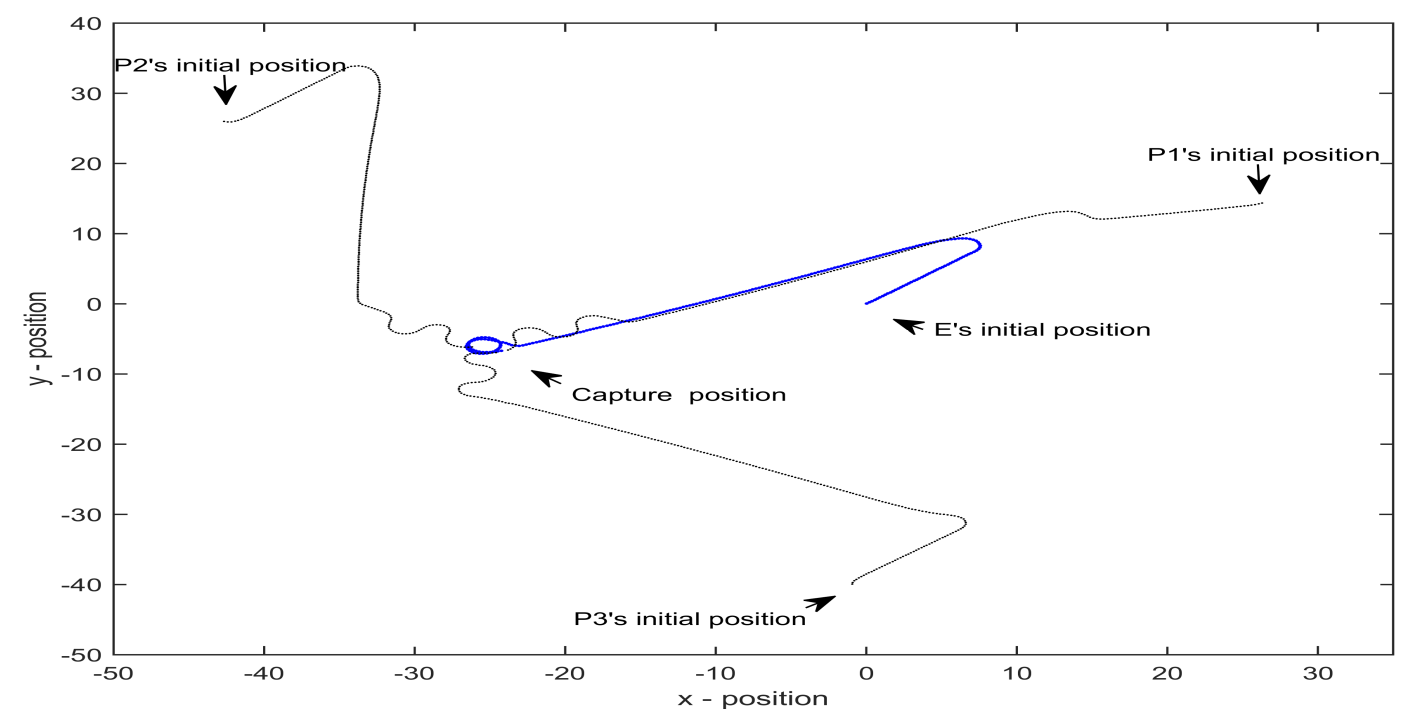

Figure 6.6: The paths of the pursuers (thin-line paths) when each pursuer $P_{o}$ learns its control strategy by the proposed algorithm; the path of the evader (thick-line path) when the evader learns its control strategy by the RGFACL algorithm. The target of the evader here is the position $(x, y)=(500,500)$. 


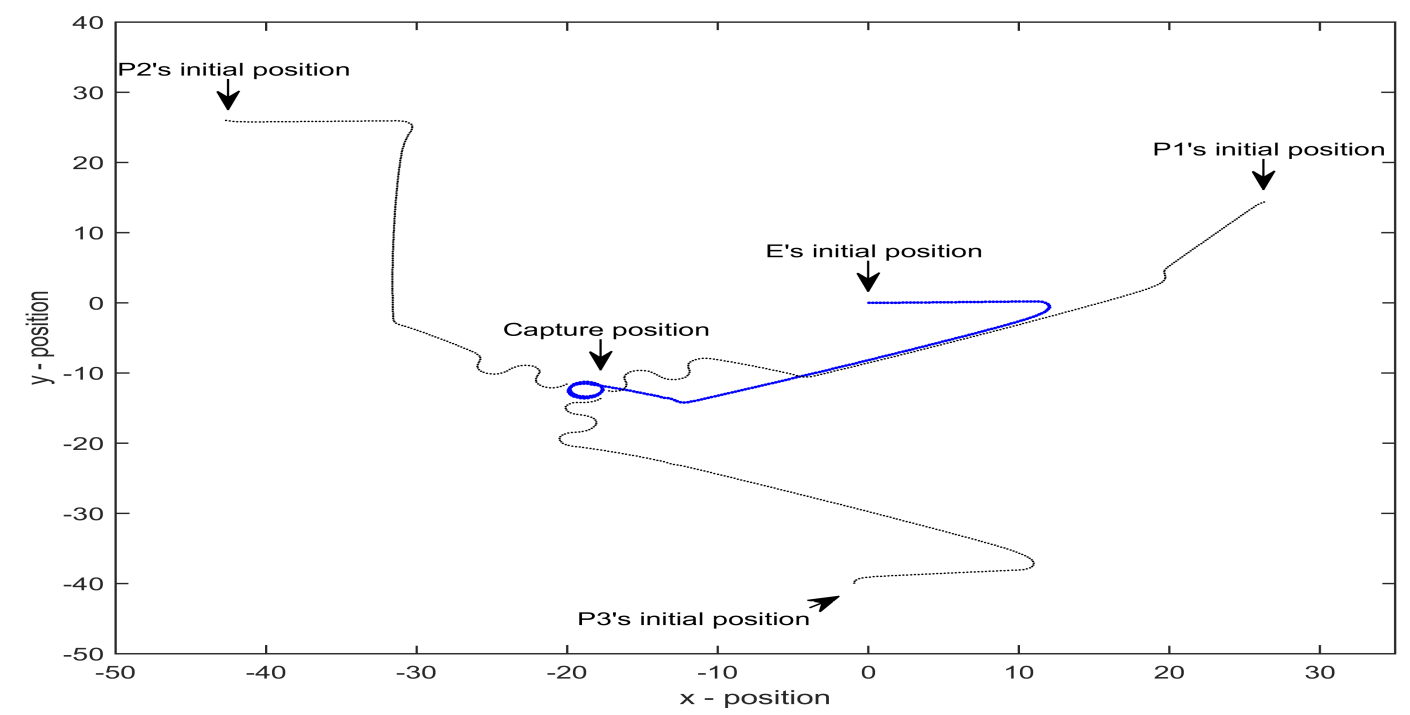

Figure 6.7: The paths of the pursuers (thin-line paths) when each pursuer $P_{o}$ learns its control strategy by the proposed algorithm; the path of the evader (thick-line path) when the evader learns its control strategy by the RGFACL algorithm. The target of the evader here is the position $(x, y)=(500,0)$.

RGFACL algorithm to tune the parameters of the FLC of each learning pursuer $P_{o}$. The proposed algorithm is a decentralized algorithm as each learning pursuer $P_{o}$ has its own learning algorithm and no communication among pursuers is required. The only information the proposed learning algorithm of the pursuer $P_{o}$ requires is the position of the superior evader. The proposed algorithm uses a formation control mechanism to construct the reward function of the learning pursuer $P_{o}$ so that the learning pursuer or the other learning pursuers can capture the superior evader. The formation control mechanism used by the proposed algorithm guarantees that the pursuers are distributed around the superior evader in order to avoid collision between pursuers. In addition, the formation control mechanism used by the proposed algorithm guarantees that the capture regions of each two adjacent pursuers overlap or at least border each other so that the capture of the superior evader will be guaranteed. The proposed algorithm is used to learn a multi-pursuer single-superior-evader 
pursuit-evasion differential game. The simulation results show the effectiveness of the proposed algorithm as the superior evader is always captured by one or some of the pursuers learning their control strategies by the proposed algorithm. In the next chapter, we develop a new decentralized fuzzy reinforcement learning algorithm that tunes the pursuers in a multi-pursuer single-superior-evader pursuit-evasion differential game where the evader has a speed that is similar to or higher than the speed of each pursuer. 


\title{
Chapter 7
}

\section{A Decentralized Fuzzy Learning}

\author{
Algorithm for Multi-Player
}

Pursuit-Evasion Differential Games with a

\author{
Single Evader Having a Speed Similar to
}

or Higher Than the Speed of Pursuers

\subsection{Introduction}

In the previous chapter, we propose a fuzzy reinforcement learning algorithm for multi-pursuer single-superior-evader pursuit-evasion differential games in which the evader and the pursuers in these games have similar speeds. In this chapter, we propose a fuzzy reinforcement learning algorithm for multi-pursuer single-superiorevader pursuit-evasion differential games in which the evader's speed in these games is similar to or higher than the speed of the pursuers. The algorithm proposed in this chapter and the algorithm proposed in the previous chapter are the first pieces of work that presents decentralized learning algorithms to capture a superior evader in pursuit-evasion differential games without using any type of discretization for the 
state or action spaces.

In this chapter, the proposed algorithm uses the well-known Apollonius circle as a mechanism to define the capture region of the learning pursuer based on its location and the location of the superior evader. Based on the defined capture region, the proposed algorithm uses a formation control approach to construct the reward function of the learning pursuer. The proposed algorithm uses this reward function to tune the FLC of the learning pursuer by the residual gradient fuzzy actor critic learning (RGFACL) algorithm proposed in [101]. The formation control approach used by the proposed algorithm guarantees that the pursuers are distributed around the superior evader in order to avoid collision between pursuers. It also makes the Apollonius circles of each two adjacent pursuers intersect or be at least tangent to each other so that the capture of the superior evader can occur. The proposed algorithm is a decentralized algorithm as no communication among the pursuers is required. The only information the proposed algorithm requires is the position and the speed of the superior evader. We evaluate the proposed algorithm over a number of multi-pursuer single-superior-evader pursuit-evasion differential games, and the results validate the proposed algorithm. The proposed algorithm is published in [139].

\subsection{Preliminary Concepts and Problem Definition}

\subsubsection{Apollonius Circles}

The Apollonius circle was first presented in [133]. Consider a single pursuit-evasion differential game with a pursuer $P$ that has a constant speed $V_{p}$ and a superior evader $E$ that has a constant speed $V_{e}$, where $V_{e}>V_{p}$. Let us also consider that the 
positions of the pursuer $P$ and the evader $E$ are $\left(x_{p}, y_{p}\right)$ and $\left(x_{e}, y_{e}\right)$, respectively. Fig. (7.1) shows the Apollonius circle created by the pursuer $P$ and the evader $E$. The pursuer captures the evader if the distance between them is less than a small amount $d_{c},\left\|\left(x_{p}, y_{p}\right)-\left(x_{e}, y_{e}\right)\right\| \leq d_{c}$. Fig. (7.1) shows the capture region of the pursuer and the evasion region of the evader. If the evader moves in a direction that is inside the capture region of the pursuer (the region covered by the angle $\angle A E B$ in Fig. (7.1)), the capture of the evader by the pursuer will be guaranteed if the pursuer is well tuned. On the other hand, if the evader moves into its evasion region (the region that is not covered by the angle $\angle A E B$ in Fig. (7.1)), the evader will be able to escape from the pursuer if the evader is well tuned.

The point $C,\left(x_{c}, y_{c}\right)$, in Fig. (7.1) is a random point that is located on the Apollonius circle and such that $\gamma=\frac{\|\overrightarrow{P C}\|}{\|\overrightarrow{E C}\|}=\frac{V_{p}}{V_{e}}$, and $\gamma<1$. The centre of the Apollonius circle, $O_{A C}$, and its radius, $R_{A C}$, can be defined as follows $[74,80]$,

$$
\begin{gathered}
O_{A C}=\left(\frac{x_{p}-\gamma^{2} x_{e}}{1-\gamma^{2}}, \frac{y_{p}-\gamma^{2} y_{e}}{1-\gamma^{2}}\right), \\
R_{A C}=\frac{\gamma \sqrt{\left(x_{p}-x_{e}\right)^{2}+\left(y_{p}-y_{e}\right)^{2}}}{1-\gamma^{2}} .
\end{gathered}
$$

As shown in Fig. (7.1), if the evader moves into the capture region of the pursuer (towards point $C$ for example) and gets captured by the pursuer, then by the triangle property, the capture condition is defined as follows $[72,73,78]$,

$$
\frac{\sin \alpha}{\sin \beta}=\frac{V_{e}}{V_{p}}
$$

where $\alpha$ is the absolute value of the angle difference between the pursuer's direction and its line of sight (LOS) to the evader, and $\beta$ is the absolute value of the angle 
difference between the evader's direction and its LOS to the pursuer.

Thus,

$$
\beta=\sin ^{-1}\left(\frac{V_{p}}{V_{e}} \sin \alpha\right)
$$

or,

$$
\beta^{*}=\sin ^{-1}\left(\frac{V_{p}}{V_{e}}\right)
$$

where $\beta^{*}=\max (\beta)$.

Hence, in a multi-player pursuit-evasion differential game with $V_{e}>V_{p}$, the condition required by the pursuer to capture the superior evader is defined as follows,

$$
\beta \leq \sin ^{-1}\left(\frac{V_{p}}{V_{e}}\right)
$$

In other words, as long as the evader's angle $\beta$ is not greater than $\beta^{*}$, the capture of the superior evader $E$ by the pursuer $P$ will be possible. Fig. (7.1) shows that the capture region of the pursuer $P$ covers $2 \beta^{*}$. In other words, the pursuer $P$ can capture the superior evader $E$ if the evader moves with $\beta \leq \beta^{*}$ from its LOS to the pursuer in the direction of either the boundary line $E A$ or the boundary line $E B$.

As can be seen in Fig. (7.1), the Apollonius circle defines the capture and escape regions of the pursuer. The boundary lines $E A$ and $E B$ separate between the capture and the scape regions of the pursuer. Fig. (7.1) also shows that when the evader moves with an angle $\beta$ such that $\beta=\beta^{*}$ in the direction of the boundary line $E A$, the pursuer has to move with an angle $\alpha=\frac{\pi}{2}$ (in counter-clockwise direction) 


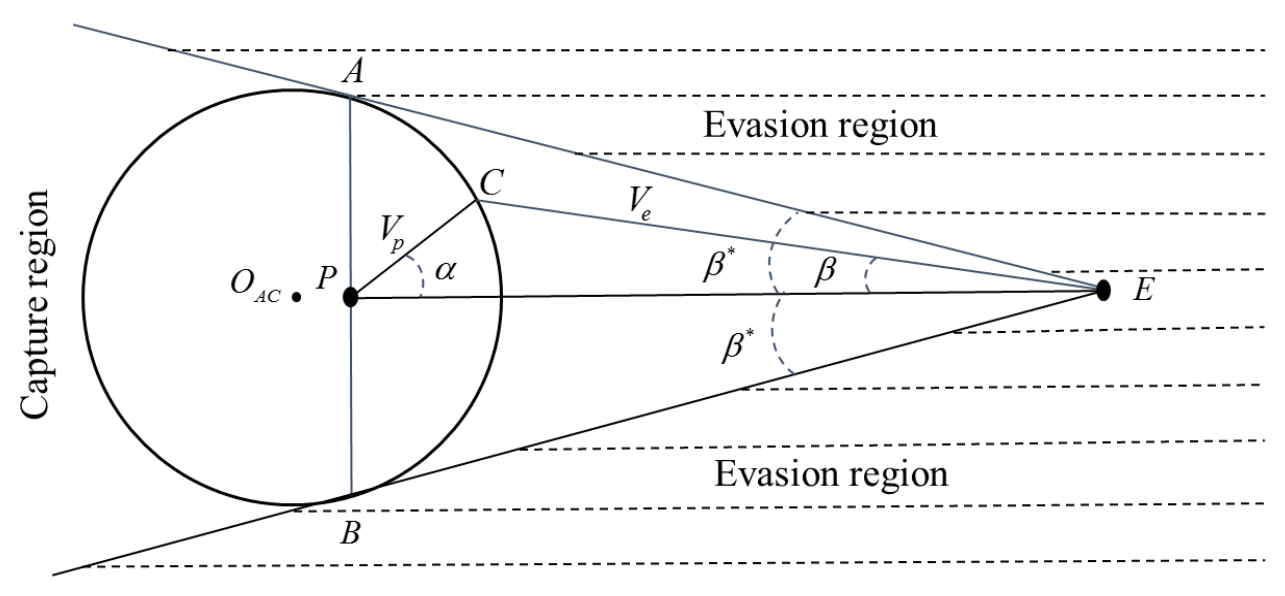

Figure 7.1: Apollonius circle created by the pursuer $\mathrm{P}$ and the evader E.

in order to satisfy the capture condition of Eq. (7.3) and capture the evader at the point $A$. In addition, when the evader moves with an angle $\beta^{*}$ in the direction of the boundary line $E B$, the pursuer has to move with an angle $\alpha=\frac{\pi}{2}$ (in clockwise direction) in order to capture the evader at the point $B$. We define the points $A$ and $B$ as the furthest capture points (boundary capture points) as they are located on the boundary lines that separate between the capture and the escape regions of the pursuer. They also cost the pursuer the longest time to capture the evader.

\subsubsection{Problem Definition}

In this chapter, we consider a pursuit-evasion differential game with $N$ pursuers and one superior evader $E$. Each pursuer $P_{o},(o=1, \ldots, N)$, has a constant speed $V_{p_{o}}$, and all pursuers have the same speed. The evader, on the other hand, has a constant speed $V_{e}$, where $V_{e}>V_{p_{o}}$. It is important to mention here that although we only now consider multi-pursuer single-superior-evader pursuit-evasion differential games with $V_{e}>V_{p_{o}}$, our work is also applicable to multi-pursuer single-superior-evader pursuit-evasion differential games with $V_{e}=V_{p_{o}}$. This will be further discussed in 
Subsection (7.3.3). The positions of the pursuers and the evader are $\left(x_{p_{o}}(t), y_{p_{o}}(t)\right)$ and $\left(x_{e}(t), y_{e}(t)\right)$, respectively. We assume that each pursuer $P_{o}$ knows the position of the evader at time $t$. We also assume that the constant speed of the evader is known to each pursuer $P_{o}$. The capture of the superior evader occurs if the distance between the pursuer $P_{o}$ and the evader $E$ is less than or equal to a small specific amount $d_{c},\left\|\left(x_{p_{o}}, y_{p_{o}}\right)-\left(x_{e}, y_{e}\right)\right\| \leq d_{c}$.

Eq. (7.2) shows that when $\gamma$ is small (the evader is much faster than the pursuer), the radius of the Apollonius circle will be small and the evader will have more wider paths to escape. Therefore, to capture the superior evader, more pursuers are needed to surround the superior evader. The pursuers have to be distributed around the superior evader to construct a polygon whose vertices are the pursuers' positions $[72,74,78]$. The Apollonius circles of each two adjacent pursuers have to intersect or be at least tangent to each other $[74,80]$. This represents the most important condition required in a multi-pursuer single-superior-evader pursuitevasion differential game in order to capture the superior evader by one or some of the pursuers. Thus, a formation control approach that controls the position and the orientation of the pursuers around the superior evader has to be integrated with the control strategy of each pursuer. This is to guarantee that the pursuers are distributed around the superior evader and the Apollonius circles of each two adjacent pursuers intersect or are at least tangent to each other.

In a multi-pursuer single-superior-evader pursuit-evasion differential game with $V_{e}>$ $V_{p_{o}}$, each pursuer $P_{o}$ covers $2 \beta^{*}$ of the evader's movement. Thus, the minimum number of the pursuers needed to surround the superior evader in order to capture it is defined as follows $[72,74,79]$, 


$$
N=\left[\frac{2 \pi}{2 \beta^{*}}\right]_{+}=\left[\frac{2 \pi}{2 \sin ^{-1}\left(\frac{V_{p}}{V_{e}}\right)}\right]_{+}=\left[\frac{\pi}{\sin ^{-1}\left(\frac{V_{p}}{V_{e}}\right)}\right]_{+} .
$$

Thus,

$$
N=\left[\frac{\pi}{\sin ^{-1}\left(\frac{V_{p}}{V_{e}}\right)}\right]_{+}
$$

where $[.]_{+}$defines the smallest integer number that is greater than or equal to [.].

From Eq. (7.7), we have

$$
N \geq \frac{\pi}{\sin ^{-1}\left(\frac{V_{p}}{V_{e}}\right)}
$$

Thus,

$$
\sin ^{-1}\left(\frac{V_{p}}{V_{e}}\right) \geq \frac{\pi}{N}
$$

or,

$$
\frac{V_{p}}{V_{e}} \geq \sin \left(\frac{\pi}{N}\right)
$$

Hence, if $\frac{V_{p}}{V_{e}} \geq \sin \left(\frac{\pi}{N}\right)$ and the Apollonius circles of each two adjacent pursuers intersect or are at least tangent to each other all the time, there will be at least one pursuer that would satisfy the capture condition of Eq. (7.3) and will then be able to capture the superior evader. 


\subsection{The Proposed Algorithm}

In this work, we develop a decentralized learning algorithm for multi-pursuer single-superior-evader pursuit-evasion differential games, where the speed of the evader is similar to or higher than the speed of each pursuer. This is the first time that anyone has shown that they can develop a system of learning to capture a superior evader in a pursuit-evasion differential game without using any type of discretization for the state or the action space. Our objective in this work is to make the pursuers in a multi-pursuer single-superior-evader pursuit-evasion differential game learn their strategies so that one or some of the learning pursuers can capture the superior evader. Although different fuzzy learning algorithms that can be applied to pursuit-evasion differential games are proposed in the literature [99-101], the proposed algorithm uses the RGFACL algorithm proposed in [101] to tune the input and the output parameters of the fuzzy logic controller (FLC) of the learning pursuer $P_{o}$. This is because the RGFACL algorithm is shown in [101] to be more robust and has a quicker convergence speed than the other (FACL and QLFIS) algorithms.

The proposed algorithm is a decentralized algorithm as each learning pursuer $P_{o}$ has its own learning algorithm and no communication among the pursuers is required. The only information the proposed learning algorithm of the pursuer $P_{o}$ requires is the position and the speed of the superior evader. The proposed algorithm uses the well-known Apollonius circle to define the capture region of the learning pursuer $P_{o}$ based on its location and the location of the superior evader. The reward function of the learning pursuer $P_{o}$ is defined based on its capture region and a developed formation control approach. This will be further discussed later on in this section. 
This reward function is used in the tuning mechanism of the FLC of the learning pursuer $P_{o}$. The formation control mechanism used in the reward function of the learning pursuer $P_{o}$ guarantees that the pursuers are distributed around the superior evader in order to avoid collision between the pursuers. In addition, the formation control mechanism makes the Apollonius circles of each two adjacent pursuers intersect or be at least tangent to each other so that the capture of the superior evader can occur.

\subsubsection{The Action $u_{p_{o}}$ of Pursuer $P_{o}$}

The action $u_{p_{o}}$ of the learning pursuer $P_{o}$ is defined as in Eq. (6.13) in Subsection (6.3.1) as follows,

$$
u_{p_{o}}=\theta_{\alpha_{o}}-\theta_{p_{o}}
$$

\subsubsection{The Developed Formation Control Approach}

Different formation control approaches that are applied to multi-pursuer singlesuperior-evader pursuit-evasion differential games are presented in the literature $[73,74,78,79]$. The formation control approaches control the distributions (the relative position and the orientation) of the pursuers around the superior evader so that the superior evader can be captured by one or some of the pursuers. The formation control approach presented in [74] is a centralized approach as it requires cooperation among pursuers. This is because the pursuer that can intercept the evader has to broadcast the interception point to the other pursuers, whose responsibilities are to head up towards that interception point in order to shrink the enclosed polygon (whose vertices are the pursuers' positions) and contain the 
evader. The formation control approach presented in [79] is a decentralized approach as no communication among pursuers is required. The strategy of each pursuer, in this formation control approach, is either to intercept the evader if the evader moves into the capture region of the pursuer or to move towards a virtual target if the evader moves into the escape region of the pursuer. The virtual target is defined as the boundary capture point (either point $A$ or point $B$ in Fig. (7.1) based on the direction of the evader), where it is most likely for the evader to cross into the capture region of the pursuer. However, when $\beta_{o}>\frac{\pi}{2}$, moving towards a virtual target by the pursuer $P_{o}$ can produce a gap between the Apollonius circle of the pursuer $P_{o}$ and the Apollonius circle of the $P_{o}$ 's adjacent pursuer. The superior evader may then use this gap to escape. The formation control approach presented in $[73,78]$ is also a decentralized approach as no communication among pursuers is required. The strategy of each pursuer, when following this formation control approach, is either to intercept the superior evader if the evader moves into the capture region of the pursuer or to move in parallel with the evader if the evader moves into the escape region of the pursuer. However, this strategy guarantees the capture of the superior evader only in the case that the superior evader and the pursuers have the same speed $\left(V_{e}=V_{p_{o}}\right)$. When $V_{e}>V_{p_{o}}$, this strategy may fail to make one or some of the pursuers capture the superior evader.

The formation control approach proposed in this work is a modified version of the formation control approaches presented in $[73,78,79]$. The proposed formation control approach used in this work is a decentralized approach. The strategy of the pursuer $P_{o}$, in this formation control approach, is to intercept the evader if the evader moves into the capture region of the pursuer. However, if the evader moves into the escape region of the pursuer, the strategy of the pursuer $P_{o}$ is either to move to a virtual target or to move in parallel with the evader. The pursuer $P_{o}$ moves to 
a virtual target if the angle $\beta_{o}$ is such that $\sin ^{-1}\left(\frac{V_{p_{o}}}{V_{e}}\right)<\beta_{o} \leq \frac{\pi}{2}$, where the virtual target is the boundary capture point (either point $A$ or point $B$ in Fig. (7.1)). On the other hand, the pursuer $P_{o}$ moves in parallel with the evader if the angle $\beta_{o}$ is

such that $\beta_{o}>\frac{\pi}{2}$. This formation control approach will guarantee that the pursuers are distributed around the superior evader in order to avoid collision between pursuers. This formation control approach will also shrink the enclosed polygon constructed by the pursuers and make the pursuers contain the evader so that the Apollonius circles of each two adjacent pursuers intersect or are at least tangent to each other. This will make one or some of the pursuers capture the superior evader.

\subsubsection{The Reward Function $r_{o}$ of Pursuer $P_{o}$}

In this work, we set the rewards of the learning pursuer $P_{o}$ based on the developed formation control mechanism illustrated in the last subsection so that one or some of the learning pursuers can capture the superior evader. The speed of the pursuer $P_{o}$ is defined by $V_{p_{o}}$, and the speed of the evader $E$ is defined by $V_{e}$. We assume that the speed of the evader is known to each pursuer. At each sampling period, the inputs to the FLC of the pursuer $P_{o}$ are the angle $\beta_{o}$ and its derivative $\dot{\beta}_{o}$. Based on its inputs, the FLC of the pursuer $P_{o}$ selects an angle $\alpha_{o}$ which is then used to calculate the action $u_{p_{o}}$ as in Eq. (7.9).

The pursuer $P_{o}$ will be rewarded based on the region that the evader moves into; the capture region of the pursuer $P_{o}$ or the evasion region of the evader shown in Fig. (7.1). It is important to mention here that the capture and escape regions of the pursuer $P_{o}$ are updated every time step. Consequently, the reward function of the pursuer $P_{o}$ will change every time step, depending on the angle $\alpha_{o}$ selected by the pursuer $P_{o}$. We will first describe how to reward the pursuer $P_{o}$ when the evader 
moves into the capture region of the pursuer $P_{o}$. That is, the evader moves with an angle $\beta_{o}$ such that $\beta_{o} \leq \sin ^{-1}\left(\frac{V_{p_{o}}}{V_{e}}\right)$. The reward function of the pursuer $P_{o}$, in this case, is constructed based on the triangle property defined in Eq. (7.3). We use the capture equation defined in Eq. (7.3) as a mechanism to reward the learning pursuer $P_{o}$ at every time step. That is, if the angle $\alpha_{o}$ selected by the pursuer $P_{o}$ satisfies the triangle property (the capture equation) of Eq. (7.3), the pursuer $P_{o}$ will be rewarded with a positive payoff ( +1 for example). On the other hand, if the angle $\alpha_{o}$ selected by the pursuer $P_{o}$ does not satisfy the triangle property (capture equation) of Eq. (7.3), the pursuer $P_{o}$ will be punished with a negative payoff ( -1 for example). This is shown in Fig. (7.2-a). Hence, the reward function of the pursuer $P_{o}$ when the evader moves into the capture region of the pursuer $P_{o}$ is defined as follows,

$$
r_{o}= \begin{cases}+1 \quad \text { if } \quad \alpha_{o} \in\left[\chi-\epsilon_{a}, \chi+\epsilon_{a}\right] \& \beta_{o} \leq \sin ^{-1}\left(\frac{V_{p_{o}}}{V_{e}}\right) \\ -1 \quad \text { otherwise, }\end{cases}
$$

where $\chi=\sin ^{-1}\left[\frac{V_{e}}{V_{p_{o}}} \sin \left(\beta_{o}\right)\right]$, and $\epsilon_{a}$ is a very small constant that defines the angle tolerance.

On the other hand, when the evader moves into its evasion region, the pursuer $P_{o}$ will not be rewarded based on the capture equation defined in Eq. (7.3). In this case, the evader moves with an angle $\beta_{o}$ such that $\beta_{o}>\sin ^{-1}\left(\frac{V_{p_{o}}}{V_{e}}\right)$. Thus, the pursuer $P_{o}$ has to work with the other pursuers to surround (enclose) the evader. This can be done by following the developed formation control illustrated in the last subsection. Thus, the strategy of the pursuer $P_{o}$, in this case, is either to move to a virtual target (the boundary capture point) or to move in parallel with the evader. The pursuer $P_{o}$ has to move to a virtual target (the boundary capture point $A$ or $B$ ) if the evader moves with an angle $\beta_{o}$ such that $\sin ^{-1}\left(\frac{V_{p_{o}}}{V_{e}}\right)<\beta_{o} \leq \frac{\pi}{2}$. On the other hand, the pursuer 
$P_{o}$ has to move in parallel with the evader if the angle $\beta_{o}$ is such that $\beta_{o}>\frac{\pi}{2}$. We will first define the reward function of the pursuer $P_{o}$ when the evader moves into the escape region of the pursuer $P_{o}$ with an angle $\beta_{o}$ such that $\sin ^{-1}\left(\frac{V_{p_{o}}}{V_{e}}\right)<\beta_{o} \leq \frac{\pi}{2}$. As illustrated in Subsection (7.2.1), the pursuer moves to the boundary capture points (either point $A$ and $B$ ) when the angle $\alpha_{o}$ selected by the pursuer $P_{o}$ is such that $\alpha_{o}=\frac{\pi}{2}$. This is shown in Fig. (7.2-b) and Fig. (7.2-c). Thus, the reward function in this case is defined as follows,

$$
r_{o}= \begin{cases}+1 \quad \text { if } \quad \alpha_{o} \in\left[\frac{\pi}{2}-\epsilon_{a}, \frac{\pi}{2}+\epsilon_{a}\right] \& \sin ^{-1}\left(\frac{V_{p_{o}}}{V_{e}}\right)<\beta_{o} \leq \frac{\pi}{2} \\ -1 \quad & \text { otherwise. }\end{cases}
$$

When the evader, on the other hand, moves into the escape region of the pursuer $P_{o}$ with an angle $\beta_{o}$ such that $\beta_{o}>\frac{\pi}{2}$, the pursuer $P_{o}$ has to move in parallel with the evader. Thus, if the angle $\alpha_{o}$ selected by the pursuer $P_{o}$ makes the pursuer $P_{o}$ move in parallel with the evader, the pursuer will be rewarded; otherwise the pursuer $P_{o}$ will be punished. To make the pursuer $P_{o}$ move in parallel with the evader, the angle $\alpha_{o}$ selected by the pursuer $P_{o}$ has to be chosen such that $\alpha_{o}+\beta_{o}=\pi$. This is shown in Fig. (7.2-d). Thus, the reward function of the pursuer $P_{o}$ when the evader moves into its evasion region with an angle $\beta_{o}$ such that $\beta_{o}>\frac{\pi}{2}$ is defined as follows,

$$
r_{o}=\left\{\begin{array}{lll}
+1 & \text { if } \quad \alpha_{o}+\beta_{o} \in\left[\pi-\epsilon_{a}, \pi+\epsilon_{a}\right] \& \beta_{o}>\frac{\pi}{2} \\
-1 & \text { otherwise }
\end{array}\right.
$$

Hence, from Eq. (7.10) to Eq. (7.12), the reward function of the pursuer $P_{o}$ when the evader moves into either the capture region of the pursuer $P_{o}$ or its evasion region can be defined as follows, 

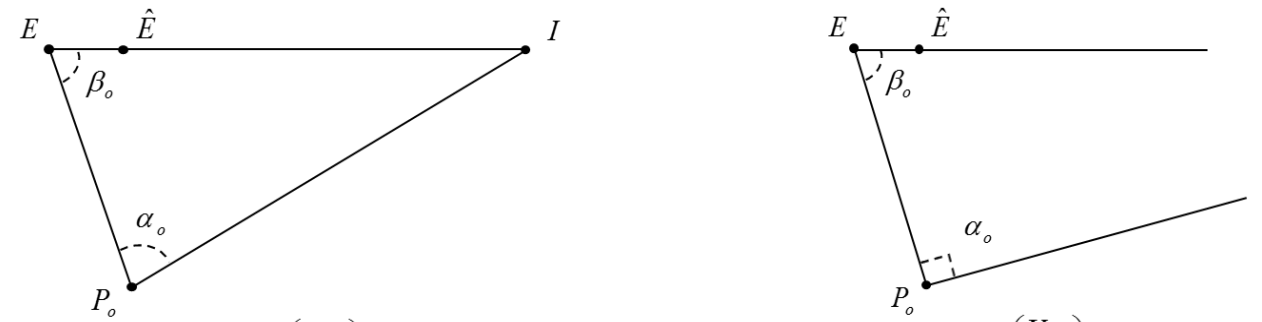

(a): $\beta_{o} \leq \sin ^{-1}\left(\frac{V_{p_{o}}}{V_{e}}\right)$

(b): $\sin ^{-1}\left(\frac{V_{p_{o}}}{V_{e}}\right)<\beta_{o} \leq \frac{\pi}{2}$
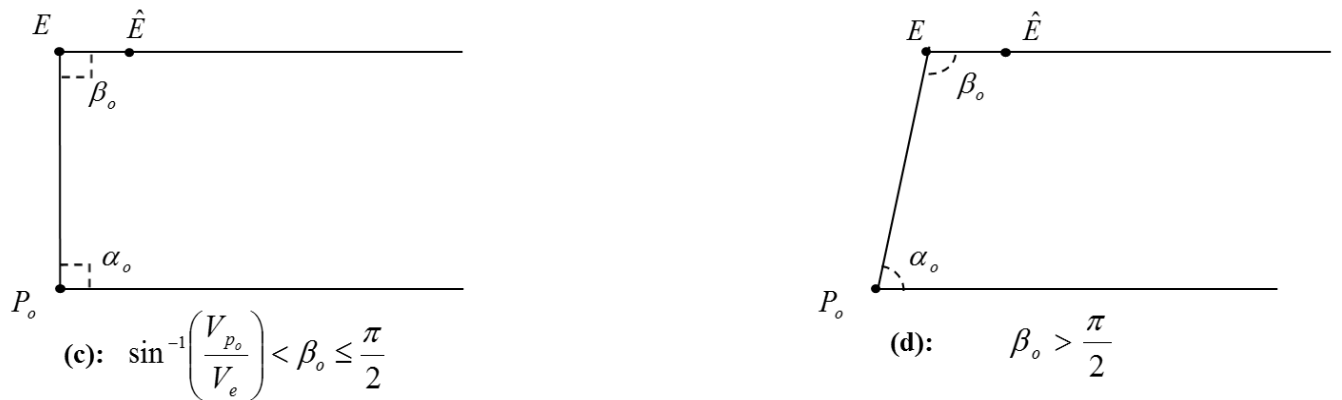

Figure 7.2: Geometric illustration of the reward function mechanism for the pursuer $P_{o}$.

$$
r_{o}=\left\{\begin{array}{lll}
+1 \quad \text { if } \quad & \alpha_{o} \in\left[\chi-\epsilon_{a}, \chi+\epsilon_{a}\right] \& \beta_{o} \leq \sin ^{-1}\left(\frac{V_{p_{o}}}{V_{e}}\right), \\
+1 \quad \text { if } \quad & \alpha_{o} \in\left[\frac{\pi}{2}-\epsilon_{a}, \frac{\pi}{2}+\epsilon_{a}\right] \& \sin ^{-1}\left(\frac{V_{p_{o}}}{V_{e}}\right)<\beta_{o} \leq \frac{\pi}{2} \\
+1 \quad \text { if } \quad & \alpha_{o}+\beta_{o} \in\left[\pi-\epsilon_{a}, \pi+\epsilon_{a}\right] \& \beta_{o}>\frac{\pi}{2}, \\
-1 & \text { otherwise, }
\end{array}\right.
$$

where $\chi=\sin ^{-1}\left[\frac{V_{e}}{V_{p_{o}}} \sin \left(\beta_{o}\right)\right]$.

Special case: When the speed of the evader is such that $V_{e}=V_{p_{o}}$, the Apollonius circle becomes a straight line and the term $\beta_{o} \leq \sin ^{-1}\left(\frac{V_{p_{o}}}{V_{e}}\right)$ in Eq. (7.13) becomes $\beta_{o} \leq \frac{\pi}{2}$. Thus, Eq. (7.13) can be rewritten as follows, 


$$
r_{o}=\left\{\begin{array}{lll}
+1 \quad \text { if } \quad \alpha_{o} \in\left[\chi-\epsilon_{a}, \chi+\epsilon_{a}\right] \& \beta_{o} \leq \frac{\pi}{2} \\
+1 \quad \text { if } \quad \alpha_{o}+\beta_{o} \in\left[\pi-\epsilon_{a}, \pi+\epsilon_{a}\right] \& \beta_{o}>\frac{\pi}{2} \\
-1 \quad & \text { otherwise. }
\end{array}\right.
$$

The strategy learned by the pursuer $P_{o}$ when using the reward function defined in Eq. (7.14) indicates that the pursuer $P_{o}$ will either intercept the evader if the evader moves into the capture region of the pursuer or move in parallel with the evader if the evader moves into the escape region of the pursuer $P_{o}$. This strategy is similar to the strategy that was presented in $[73,78]$ in the case that the superior evader and the pursuers have the same speeds (i.e. $V_{e}=V_{p_{o}}$ ). Hence, the reward function mechanism constructed based on the formation control approach developed in this work will also be applicable in the case that the superior evader and the pursuers have the same speeds.

\subsection{Simulation and Results}

In this section, we evaluate the proposed algorithm on three different multi-pursuer single-superior-evader pursuit-evasion differential games where the speed of the evader is similar to or higher than the speed of each pursuer. In each game, each pursuer $P_{o}$ is learning its control strategy so that one or some of the learning pursuers can capture the superior evader. The evader is also learning its control strategy so that it can reach a specific target $\left(x_{e}^{T}, y_{e}^{T}\right)$ before it is captured by one or some of the pursuers. It is important to mention here that our objective is not to design an optimal strategy for the superior evader. Our objective is only to evaluate our algorithm when the evader is an intelligent superior evader. Thus, we assume that 
the priority of learning for the evader is to learn how to reach its target. However, if the distance between the evader and the nearest pursuer is less than a specific distance (tolerance distance, $d_{t o l}$ ), the priority of learning for the evader becomes to escape from that pursuer.

\subsubsection{Simulation Setup}

As illustrated in the previous section, for each pursuer $P_{o},(o=1, \ldots, N)$, we define the angle $\beta_{o}$ as the absolute value of the angle difference between the evader's direction and its LOS to the pursuer $P_{o}$. In addition, we define the state $\mathbf{s}_{t}$ for the pursuer $P_{o}$ by two input variables which are the angle $\beta_{o}$ and its derivative $\dot{\beta}_{o}$. Five Gaussian membership functions (MFs) are used to define the fuzzy sets of each input to the FISs of the proposed learning algorithm of each pursuer $P_{o}$. On the other hand, the state $\mathbf{s}_{t}$ of the evader is defined by two input variables, $\delta_{e}$ and its derivative $\dot{\delta}_{e}$, where $\delta_{e}$ is the angle difference between the direction of the evader and the direction of its target $\left(x_{e}^{T}, y_{e}^{T}\right)$. However, if the distance between the evader and the nearest pursuer, $d_{p_{o}}$, is less than the tolerance distance $d_{t o l}, \delta_{e}$ is defined as the angle difference between the direction of the evader and the direction of the LOS of that nearest pursuer to the evader. Thus, the reward function of the superior evader is defined as follows,

$$
r_{e}=\left\{\begin{aligned}
\Delta_{e}(t) \quad \text { if } \quad d_{p_{o}}>d_{t o l}, \\
-\Delta_{p_{o}}(t) \quad \text { if } \quad d_{p_{o}} \leq d_{t o l} \& d_{p_{o}} \leq d_{p_{j}} \& o \neq j,
\end{aligned}\right.
$$

where, 


$$
\begin{gathered}
\Delta_{e}(t)=D_{e}(t)-D_{e}(t+1), \\
D_{e}(t)=\left\|\left(x_{e}, y_{e}\right)-\left(x_{e}^{T}, y_{e}^{T}\right)\right\|, \\
d_{p_{o}}=\left\|\left(x_{p_{o}}, y_{p_{o}}\right)-\left(x_{e}, y_{e}\right)\right\|, \\
\Delta_{p_{o}}(t)=D_{p_{o}}(t)-D_{p_{o}}(t+1), \\
D_{p_{o}}(t)=\left\|\left(x_{p_{o}}, y_{p_{o}}\right)-\left(x_{e}, y_{e}\right)\right\|, \\
d_{p_{j}}=\left\|\left(x_{p_{j}}, y_{p_{j}}\right)-\left(x_{e}, y_{e}\right)\right\| .
\end{gathered}
$$

Five Gaussian membership functions (MFs) are used to define the fuzzy sets of each input to the FISs of the RGFACL algorithm of the evader. The wheelbases and the maximum steering angles of the pursuers and the evader are set as follows, $L_{p_{o}}=L_{e}=0.5 \mathrm{~m}$ and $u_{p_{o}}^{\max }=u_{e}^{\max }=0.8 \mathrm{rad}$. The parameters of the learning algorithms of the pursuers and the evader are set as those parameters in [101]. The sampling time is defined as $T=0.05 \mathrm{~s}$, whereas the capture radius is defined as $d_{c}=0.5 \mathrm{~m}$. The tolerance distance is defined as $d_{t o l}=10 \mathrm{~m}$. The number of episodes is set to 200, whereas the number of steps (in each episode) is set to 2000.

Game 1: In this game, the speed of the evader is equal to the speed of each pursuer 
(i.e. $V_{e}=V_{p_{o}}=1 \mathrm{~m} / \mathrm{s}$ ). We use three pursuers, (i.e. $N=3$ and $o=1,2,3$ ). The evader starts its motion from the position $\left(x_{e}, y_{e}\right)=(0,0)$ with an initial orientation $\theta_{e}=0 \mathrm{rad}$. The pursuer $P_{1}$ starts its motion from a random position at $d_{1} \angle \theta_{d_{1}} ; d_{1}$ is the distance between the pursuer $P_{1}$ and the origin $O=(0,0)$, and $\theta_{d_{1}}$ is the angle that describes the direction of the vector $\overrightarrow{O P_{1}}$. Thus, the random position of the pursuer $P_{1}$ is defined as $\left(x_{p_{1}}, y_{p_{1}}\right)=\left(d_{1} \cos \theta_{d_{1}}, d_{1} \sin \theta_{d_{1}}\right)$. The initial orientation of the pursuer $P_{1}$ is defined as $\theta_{p_{1}}=\tan ^{-1}\left(\frac{-y_{p_{1}}}{-x_{p_{1}}}\right)$. Similarly, the pursuers $P_{2}$ and $P_{3}$ start their motions from random positions but taking into account the angle distributions of the pursuers around the evader required by the formation control. Since we have three pursuers, we define the initial positions of the pursuers $P_{2}$ and $P_{3}$ as $\left(x_{p_{2}}, y_{p_{2}}\right)=\left(d_{2} \cos \left(\theta_{d_{1}}+2 \pi / 3\right), d_{2} \sin \left(\theta_{d_{1}}+2 \pi / 3\right)\right)$ and $\left(x_{p_{3}}, y_{p_{3}}\right)=\left(d_{3} \cos \left(\theta_{d_{1}}+4 \pi / 3\right), d_{3} \sin \left(\theta_{d_{1}}+4 \pi / 3\right)\right)$, respectively. The distances $d_{1}, d_{2}$ and $d_{3}$ are randomly selected. The initial orientations of the pursuers $P_{2}$ and $P_{3}$ are defined as $\theta_{p_{2}}=\tan ^{-1}\left(\frac{-y_{p_{2}}}{-x_{p_{2}}}\right)$ and $\theta_{p_{3}}=\tan ^{-1}\left(\frac{-y_{p_{3}}}{-x_{p_{3}}}\right)$, respectively.

Game 2: In this game, the speed of the evader is higher than the speed of each pursuer. We set the speed of the superior evader as $V_{e}=1.1 \mathrm{~m} / \mathrm{s}$ and the speed of each pursuer $P_{o}$ as $V_{p_{o}}=1 \mathrm{~m} / \mathrm{s}$. Thus, the number of the pursuers required to capture the superior evader in this game is set based on Eq. (7.7) as follows,

$$
N=\left[\frac{\pi}{\sin ^{-1}\left(\frac{V_{p}}{V_{e}}\right)}\right]_{+}=\left[\frac{\pi}{\sin ^{-1}\left(\frac{1}{1.1}\right)}\right]_{+}=[2.75]_{+}=3 .
$$

The initial positions and orientations of the superior evader and the pursuers are set as in Game 1.

Game3: In this game, the speed of the evader is also higher than the speed of each pursuer. We set the speed of the superior evader as $V_{e}=1.2 \mathrm{~m} / \mathrm{s}$ and the speed 
of each pursuer $P_{o}$ as $V_{p_{o}}=1 \mathrm{~m} / \mathrm{s}$. Thus, the number of the pursuers required to capture the superior evader in this game is set based on Eq. (7.7) as follows,

$$
N=\left[\frac{\pi}{\sin ^{-1}\left(\frac{V_{p}}{V_{e}}\right)}\right]_{+}=\left[\frac{\pi}{\sin ^{-1}\left(\frac{1}{1.2}\right)}\right]_{+}=[3.19]_{+}=4
$$

In this game, the evader starts its motion from the position $\left(x_{e}, y_{e}\right)=(0,0)$ with an initial orientation $\theta_{e}=0 \mathrm{rad}$. Likewise Game 1 and Game 2, the pursuers in Game 3 start their motions from random positions defined as follows, $\left(x_{p_{1}}, y_{p_{1}}\right)=$ $\left(d_{1} \cos \theta_{d_{1}}, d_{1} \sin \theta_{d_{1}}\right),\left(x_{p_{2}}, y_{p_{2}}\right)=\left(d_{2} \cos \left(\theta_{d_{1}}+\pi / 2\right), d_{2} \sin \left(\theta_{d_{1}}+\pi / 2\right)\right),\left(x_{p_{3}}, y_{p_{3}}\right)=$ $\left(d_{3} \cos \left(\theta_{d_{1}}+\pi\right), d_{3} \sin \left(\theta_{d_{1}}+\pi\right)\right)$, and $\left(x_{p_{4}}, y_{p_{4}}\right)=\left(d_{4} \cos \left(\theta_{d_{1}}+3 \pi / 2\right), d_{4} \sin \left(\theta_{d_{1}}+3 \pi / 2\right)\right)$.

\subsubsection{Results}

Fig. (7.3) to Fig. (7.6) show the paths of the pursuers of Game 1 when each pursuer learns its control strategy by the proposed algorithm. Fig. (7.3) to Fig. (7.6) also show the path of the evader when the evader in Game 1 learns its control strategy by the RGFACL algorithm. The initial positions of the pursuers are set as $\left(x_{p_{1}}, y_{p_{1}}\right)=40 \angle 0.5,\left(x_{p_{2}}, y_{p_{2}}\right)=50 \angle(0.5+2 \pi / 3)$ and $\left(x_{p_{3}}, y_{p_{3}}\right)=30 \angle(0.5+4 \pi / 3)$. As shown in Fig. (7.3) to Fig. (7.6), the superior evader is always captured by one or some of the pursuers learning their control strategies by the proposed algorithm. In each figure, the evader has a specific target to go to. However, when the distance between the evader and the nearest pursuer is less than the tolerance distance $d_{t o l}$, the evader's priority becomes to escape from that pursuer. However, because of the formation control mechanism used by the proposed learning algorithm, the evader is always enclosed by the pursuers until it is eventually captured by one or some of the pursuers. 
Fig. (7.7) to Fig. (7.10) show the paths of the pursuers of Game 2 when each pursuer learns its control strategy by the proposed algorithm. Fig. (7.7) to Fig. (7.10) also show the path of the evader of Game 2 when the evader learns its control strategy by the RGFACL algorithm. The initial positions of the pursuers are set as in Game 1. The figures show that the pursuers learning their control strategy by the proposed algorithm always succeed to capture the superior evader although the evader moves with a speed that is higher than the speed of the pursuers.

Fig. (7.11) to Fig. (7.14) show the results of the simulation we perform on Game 3 when the pursuers learn their control strategies by the proposed algorithm; the paths of the pursuers and the superior evader of Game 3 are shown. The initial positions of the pursuers are set as $\left(x_{p_{1}}, y_{p_{1}}\right)=30 \angle 0.5,\left(x_{p_{2}}, y_{p_{2}}\right)=50 \angle(0.5+\pi / 2)$, $\left(x_{p_{3}}, y_{p_{3}}\right)=40 \angle(0.5+\pi)$, and $\left(x_{p_{4}}, y_{p_{4}}\right)=30 \angle(0.5+3 \pi / 2)$. At each figure, the

superior evader is assigned a target $\left(x_{e}^{T}, y_{e}^{T}\right)$ to go to. When the distance between the superior evader and the nearest pursuer is less than the tolerance distance $\left(d_{t o l}=10\right.$ m), the evader's priority becomes to escape from that pursuer. Fig. (7.11) to Fig. (7.14) show that the pursuers trained by the proposed algorithm always succeed to capture the superior evader. Hence, the simulation we conduct on Game 1, Game 2, and Game 3 show the effectiveness of the proposed algorithm.

\subsection{Summary and Discussion}

In this chapter, we propose a new fuzzy reinforcement learning algorithm for multipursuer single-superior-evader pursuit-evasion differential games so that the superior evader can be captured by one or some of the learning pursuers. A "superior evader" is defined as an evader that has a speed that is equal to or higher than the speed of 


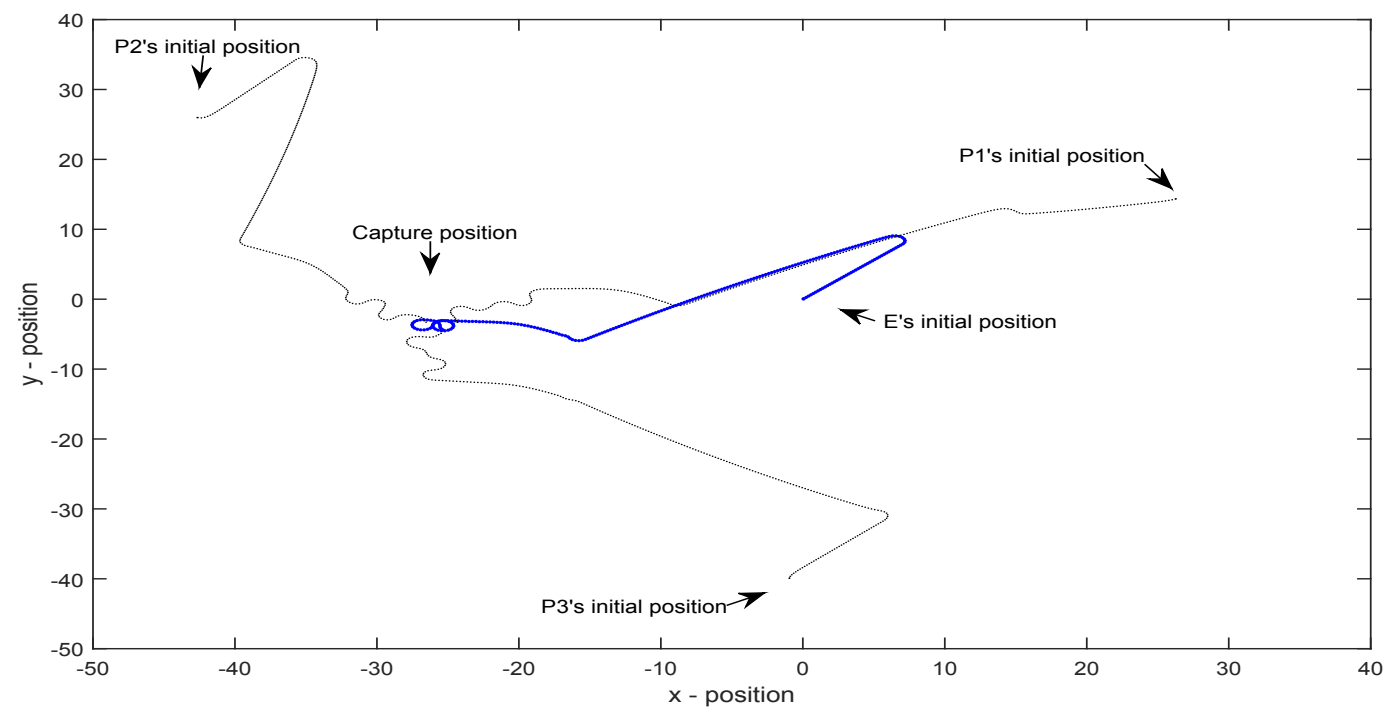

Figure 7.3: The paths of the pursuers of Game 1 (thin-line paths) when each pursuer $P_{o}$ learns its control strategy by the proposed algorithm; the path of the evader (thick-line path) when it learns its control strategy by the RGFACL algorithm. The target of the evader here is the position $\left(x_{e}^{T}, y_{e}^{T}\right)=(500,500)$.

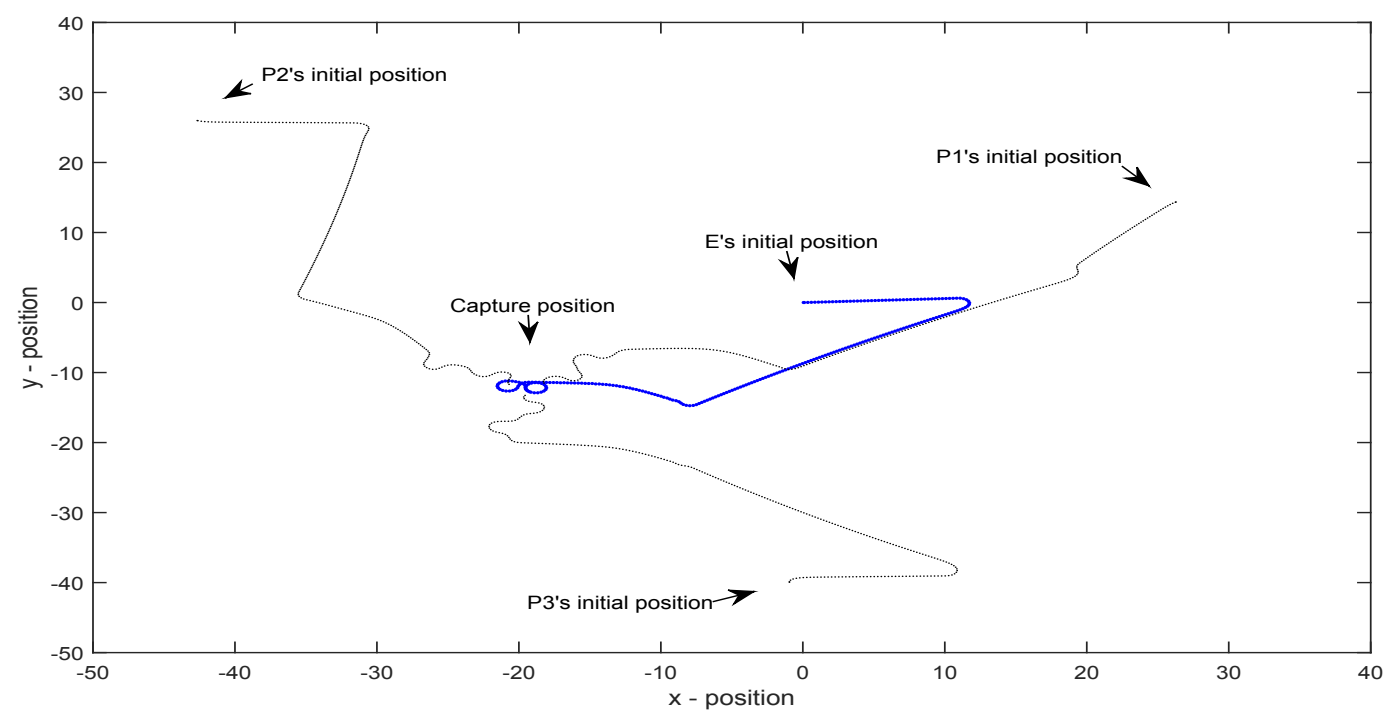

Figure 7.4: The paths of the pursuers of Game 1 (thin-line paths) when each pursuer $P_{o}$ learns its control strategy by the proposed algorithm; the path of the evader (thick-line path) when it learns its control strategy by the RGFACL algorithm. The target of the evader here is the position $\left(x_{e}^{T}, y_{e}^{T}\right)=(500,0)$. 


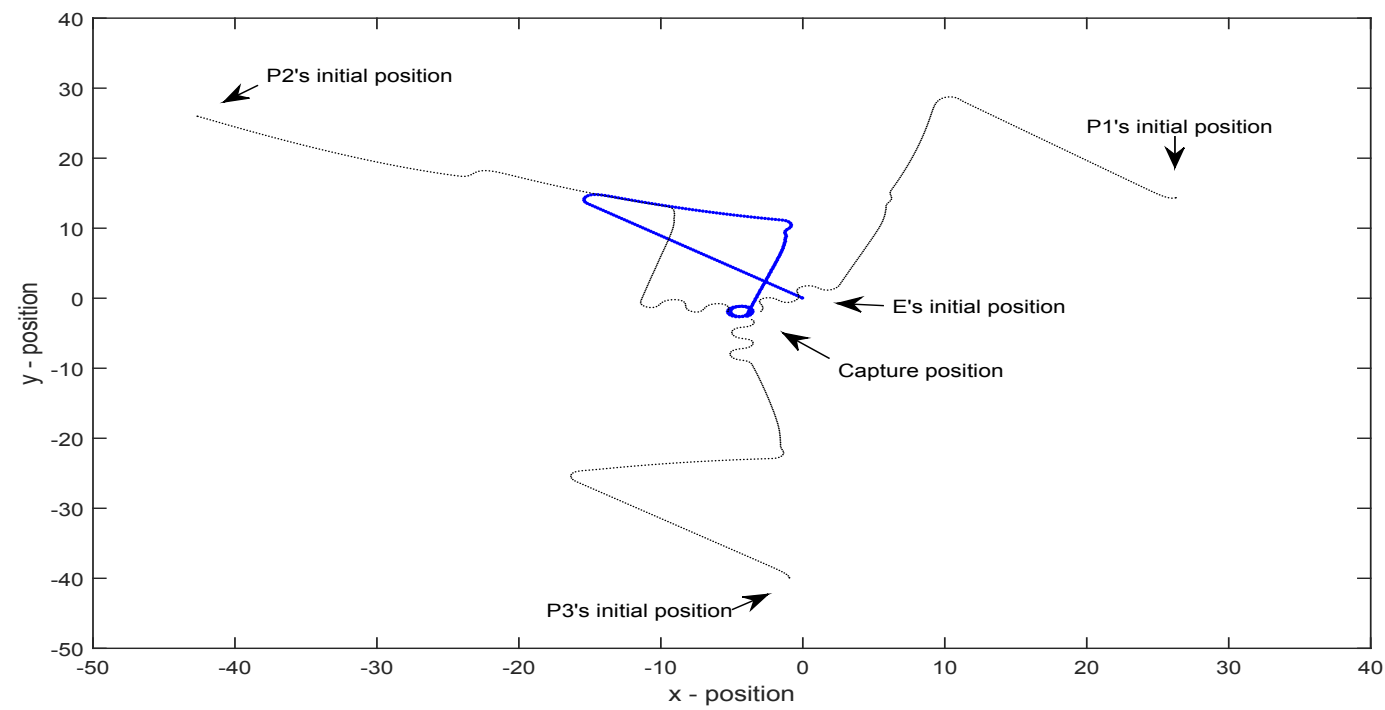

Figure 7.5: The paths of the pursuers of Game 1 (thin-line paths) when each pursuer $P_{o}$ learns its control strategy by the proposed algorithm; the path of the evader (thick-line path) when it learns its control strategy by the RGFACL algorithm. The target of the evader here is the position $\left(x_{e}^{T}, y_{e}^{T}\right)=(-500,500)$.

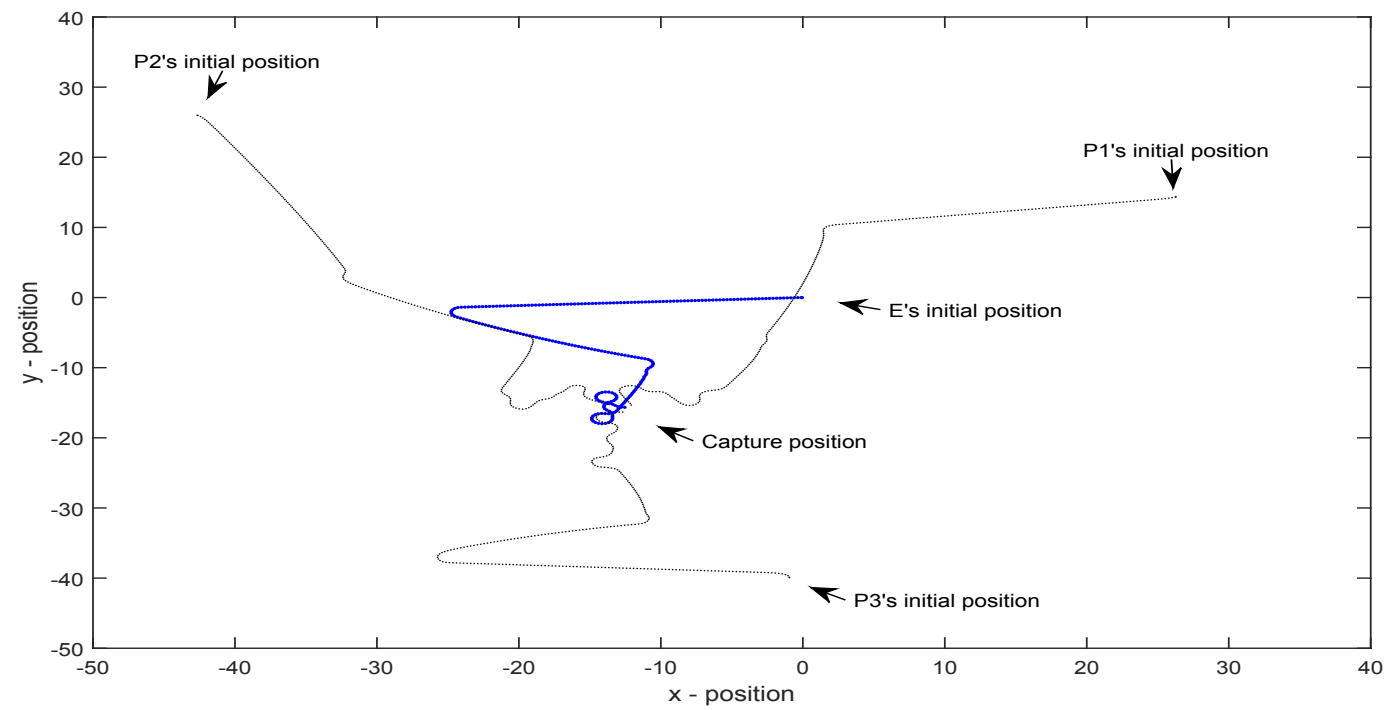

Figure 7.6: The paths of the pursuers of Game 1 (thin-line paths) when each pursuer $P_{o}$ learns its control strategy by the proposed algorithm; the path of the evader (thick-line path) when it learns its control strategy by the RGFACL algorithm. The target of the evader here is the position $\left(x_{e}^{T}, y_{e}^{T}\right)=(-500,0)$. 


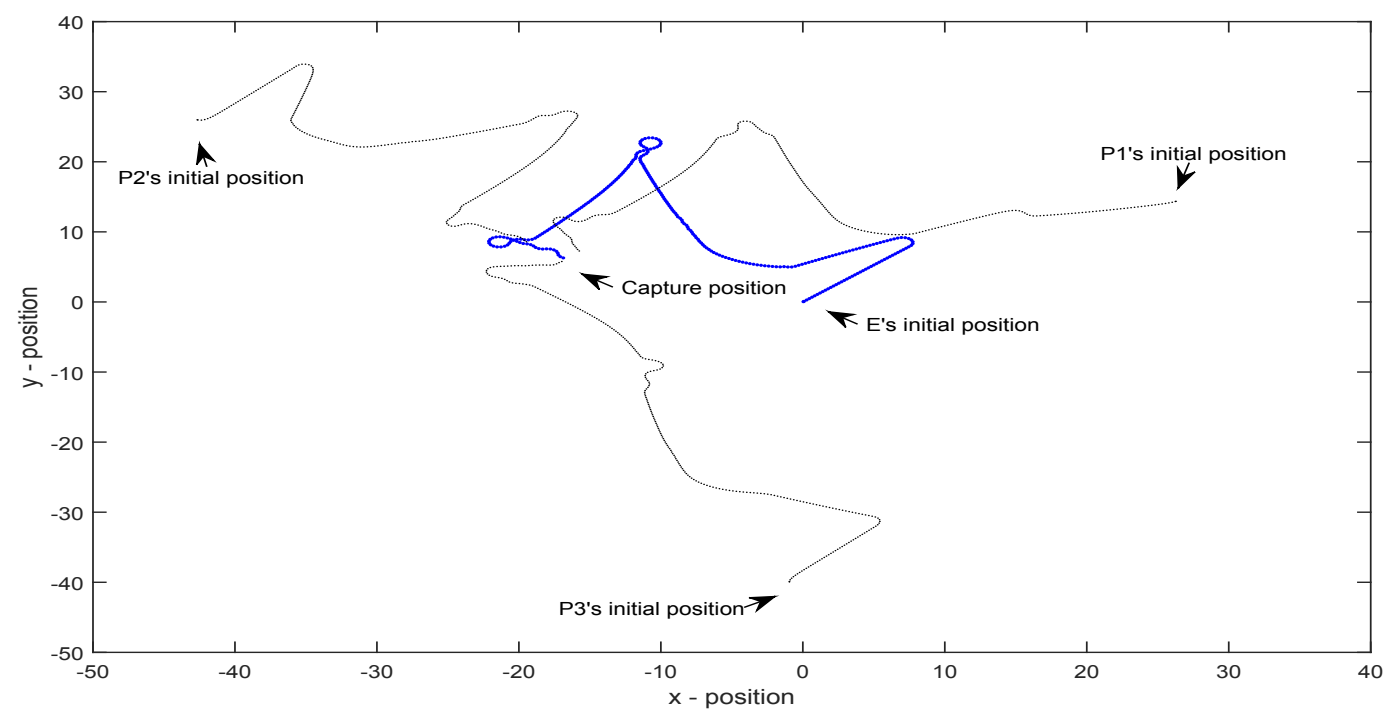

Figure 7.7: The paths of the pursuers of Game 2 (thin-line paths) when each pursuer $P_{o}$ learns its control strategy by the proposed algorithm; the path of the evader (thick-line path) when it learns its control strategy by the RGFACL algorithm. The target of the evader here is the position $\left(x_{e}^{T}, y_{e}^{T}\right)=(500,500)$.

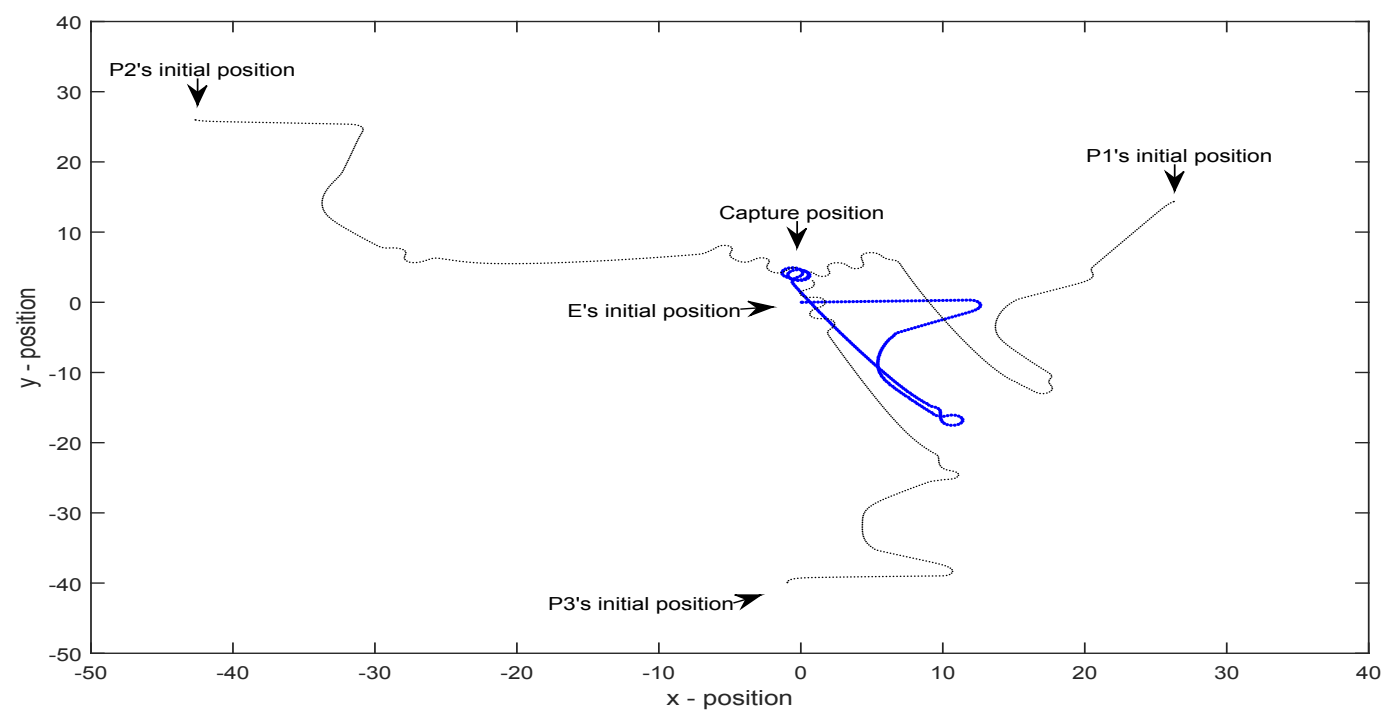

Figure 7.8: The paths of the pursuers of Game 2 (thin-line paths) when each pursuer $P_{o}$ learns its control strategy by the proposed algorithm; the path of the evader (thick-line path) when it learns its control strategy by the RGFACL algorithm. The target of the evader here is the position $\left(x_{e}^{T}, y_{e}^{T}\right)=(500,0)$. 


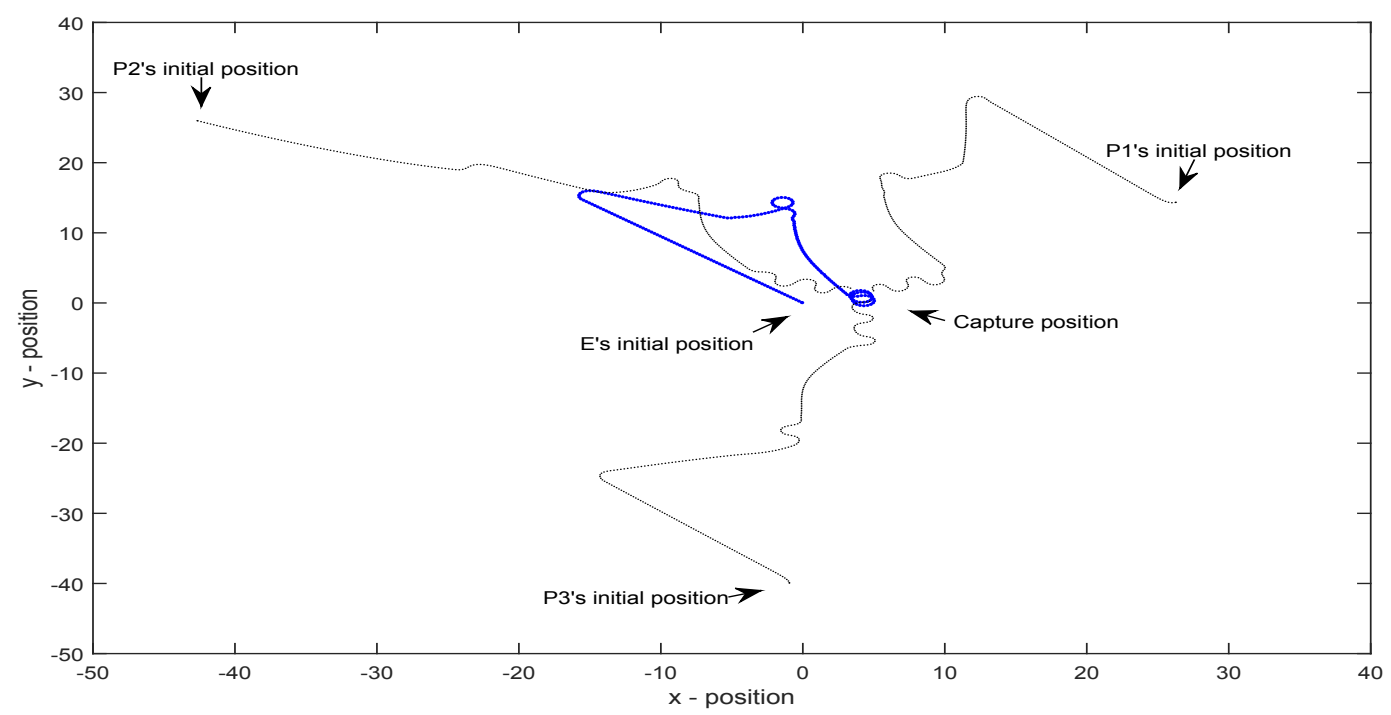

Figure 7.9: The paths of the pursuers of Game 2 (thin-line paths) when each pursuer $P_{o}$ learns its control strategy by the proposed algorithm; the path of the evader (thick-line path) when it learns its control strategy by the RGFACL algorithm. The target of the evader here is the position $\left(x_{e}^{T}, y_{e}^{T}\right)=(-500,500)$.

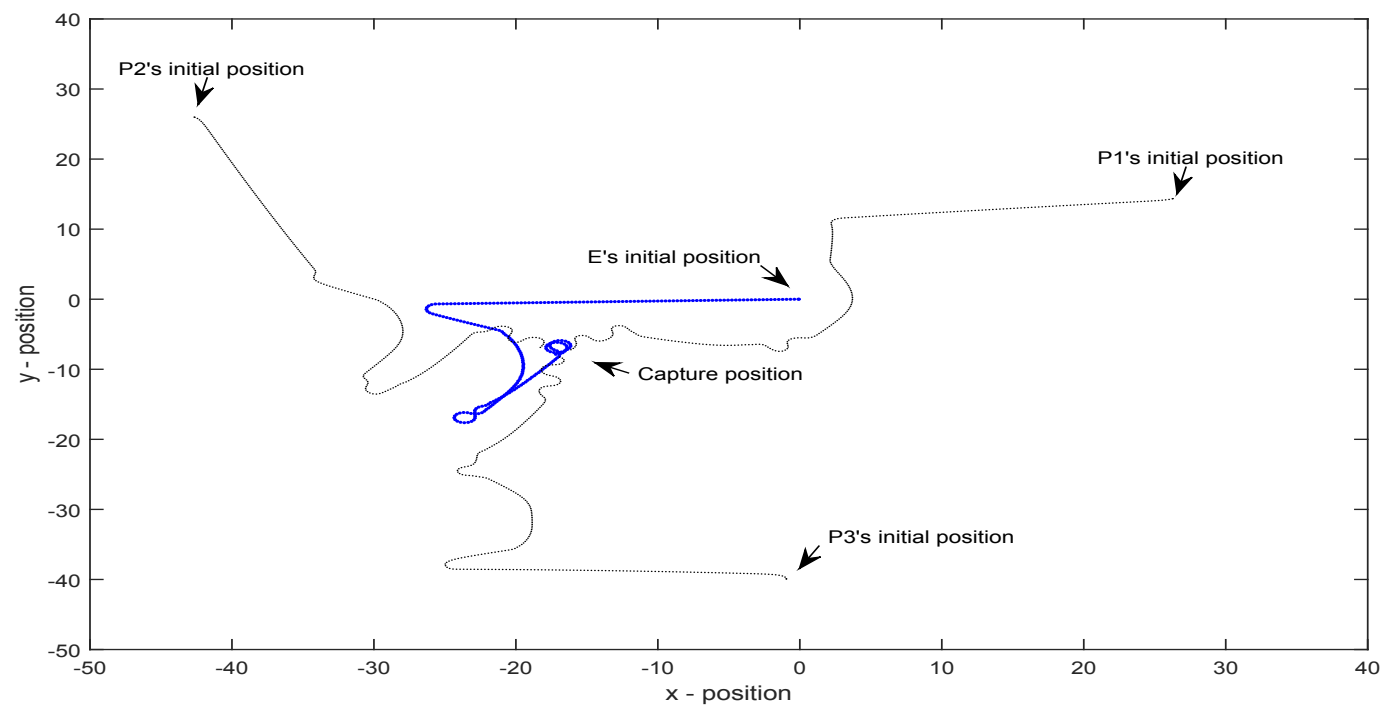

Figure 7.10: The paths of the pursuers of Game 2 (thin-line paths) when each pursuer $P_{o}$ learns its control strategy by the proposed algorithm; the path of the evader (thick-line path) when it learns its control strategy by the RGFACL algorithm. The target of the evader here is the position $\left(x_{e}^{T}, y_{e}^{T}\right)=(-500,0)$. 


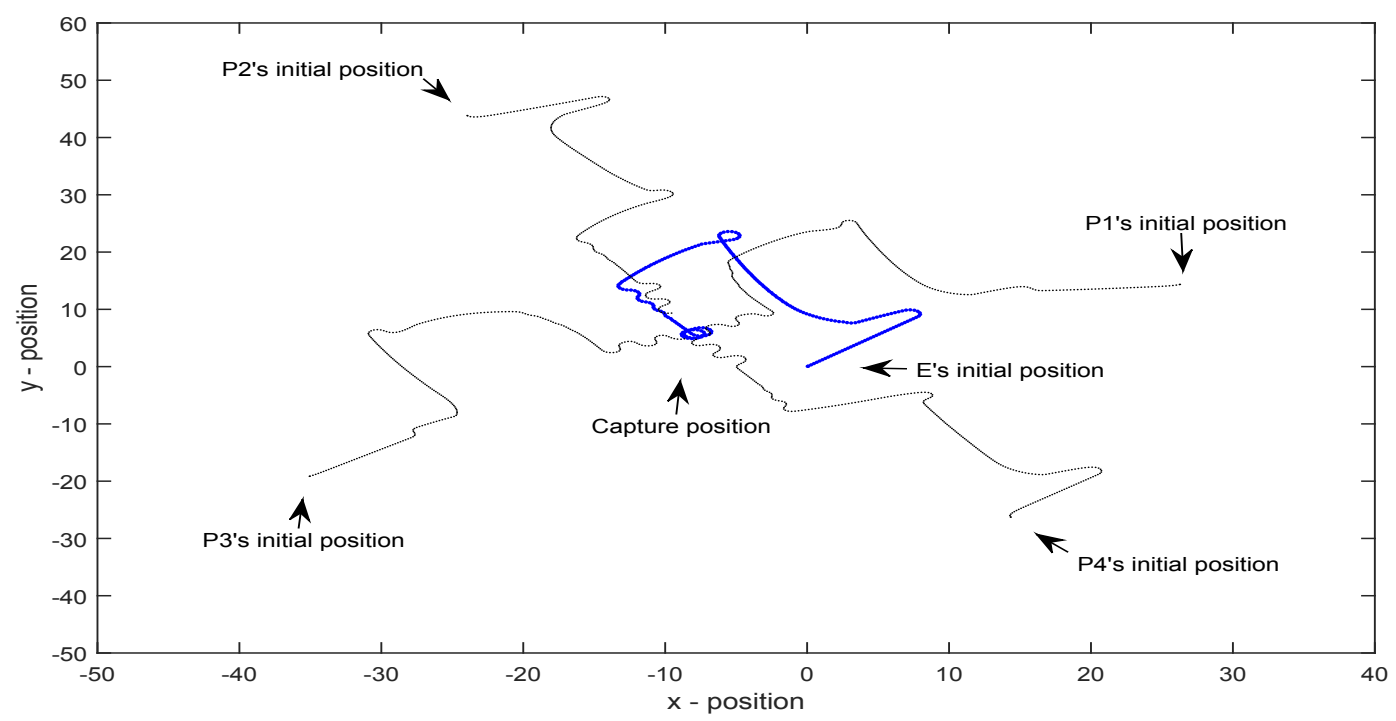

Figure 7.11: The paths of the pursuers of Game 3 (thin-line paths) when each pursuer $P_{o}$ learns its control strategy by the proposed algorithm; the path of the evader (thick-line path) when it learns its control strategy by the RGFACL algorithm. The target of the evader here is the position $\left(x_{e}^{T}, y_{e}^{T}\right)=(500,500)$.

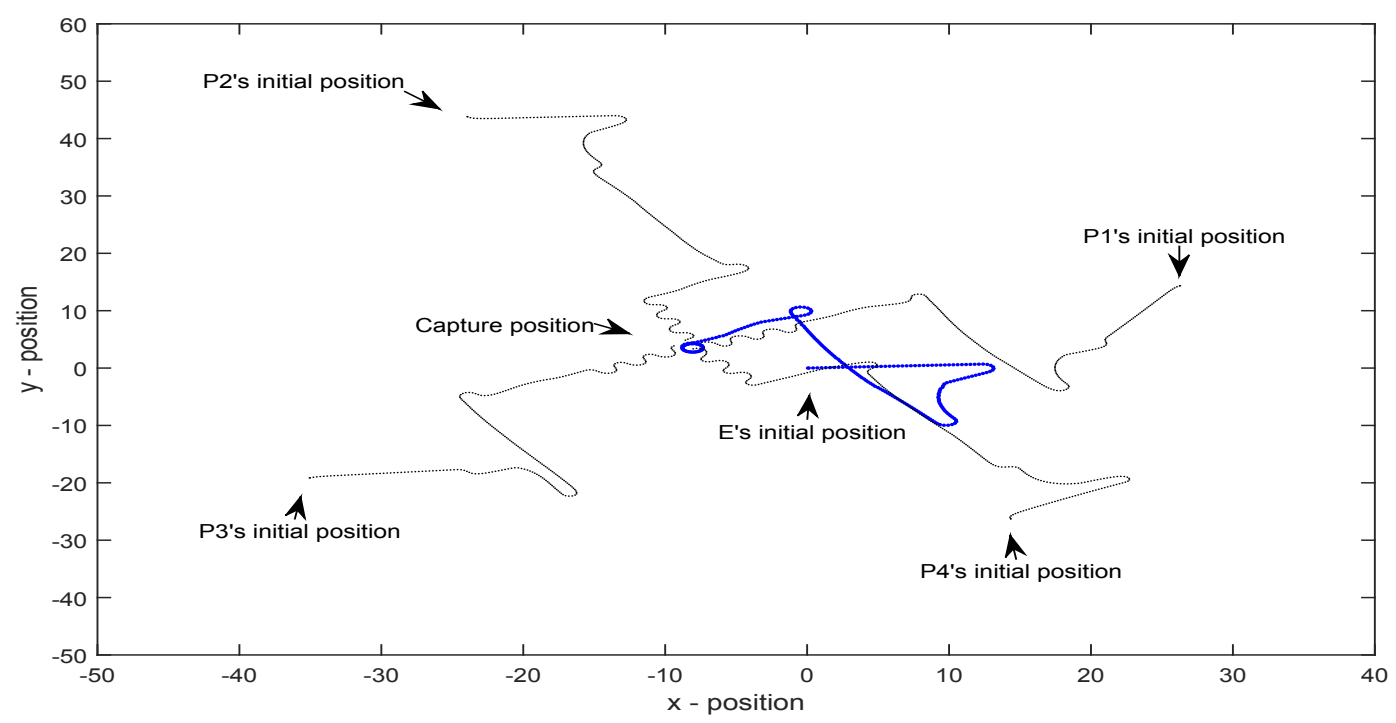

Figure 7.12: The paths of the pursuers of Game 3 (thin-line paths) when each pursuer $P_{o}$ learns its control strategy by the proposed algorithm; the path of the evader (thick-line path) when it learns its control strategy by the RGFACL algorithm. The target of the evader here is the position $\left(x_{e}^{T}, y_{e}^{T}\right)=(500,0)$. 


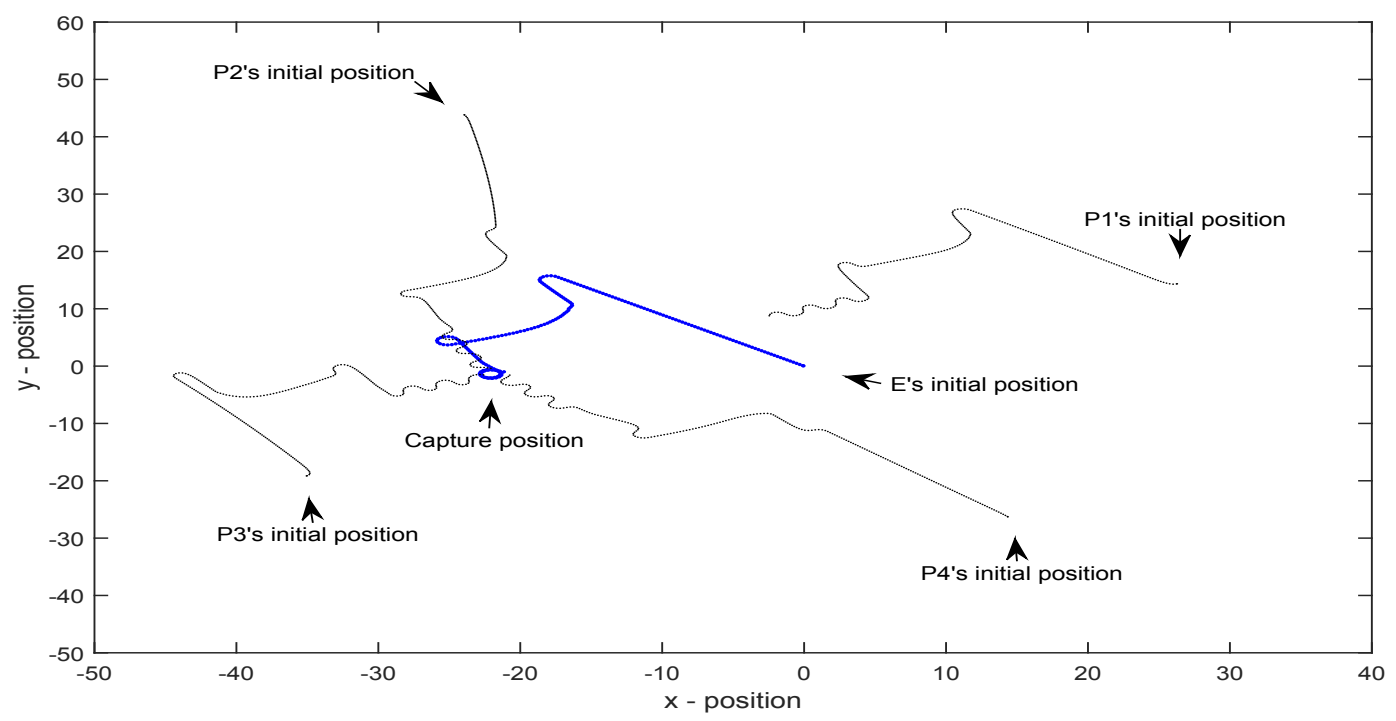

Figure 7.13: The paths of the pursuers of Game 3 (thin-line paths) when each pursuer $P_{o}$ learns its control strategy by the proposed algorithm; the path of the evader (thick-line path) when it learns its control strategy by the RGFACL algorithm. The target of the evader here is the position $\left(x_{e}^{T}, y_{e}^{T}\right)=(-500,500)$.

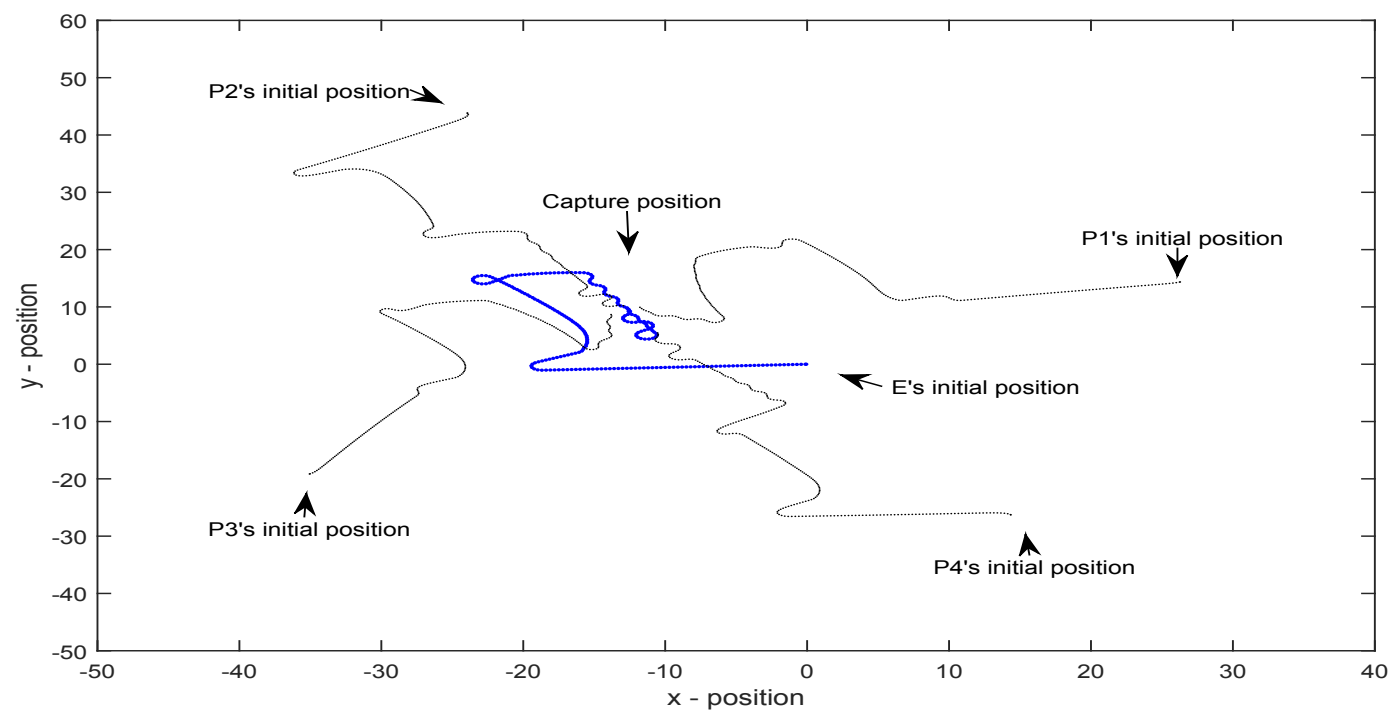

Figure 7.14: The paths of the pursuers of Game 3 (thin-line paths) when each pursuer $P_{o}$ learns its control strategy by the proposed algorithm; the path of the evader (thick-line path) when it learns its control strategy by the RGFACL algorithm. The target of the evader here is the position $\left(x_{e}^{T}, y_{e}^{T}\right)=(-500,0)$. 
each pursuer in the game. The proposed algorithm uses the well-known Apollonius circle mechanism to define the capture region of each pursuer based on its location and the location of the superior evader. The proposed algorithm uses the RGFACL algorithm to tune the FLC of each pursuer so that the pursuers learn their control strategies to capture the superior evader. A new formation control mechanism is proposed in this work and is used with the Apollonius circle mechanism to construct the reward function of each learning pursuer. The formation control mechanism used by the proposed algorithm guarantees that the pursuers are distributed around the superior evader in order to avoid collision between pursuers. The formation control mechanism used by the proposed algorithm also makes the Apollonius circles of each two adjacent pursuers intersect or are at least be tangent to each other so that the capture of the superior evader can occur. The proposed algorithm is a decentralized algorithm as no communication among the pursuers is required. The only information the proposed algorithm requires is the position and the speed of the superior evader. The proposed algorithm is used to learn different multi-pursuer single-superior-evader pursuit-evasion differential games. The simulation results show the effectiveness of the proposed algorithm. 


\section{Chapter 8}

\section{Conclusions}

In this thesis, we investigate several issues of reinforcement learning in matrix, stochastic, and differential games. The issues we investigate include:

- the problem of learning in matrix and stochastic games when each learning agent has only minimum knowledge about the underlying game and the other learning agents;

- the problem of learning in differential games where the states and actions are represented in continuous domains;

- the problem of capturing the evader, in a pursuit-evasion differential game, when the environment of the game is different from the training environment;

- the problem of capturing a superior evader in multi-pursuer pursuit-evasion differential games with a single-superior evader that has a speed equal to the speed of the pursuers;

- the problem of capturing a superior evader in multi-pursuer pursuit-evasion differential games with a single-superior evader that has a speed that is equal to or higher than the speed of each pursuer.

Our contributions to address these issues are summarized as follows, 
1. We propose two multi-agent reinforcement learning algorithms to solve the problem of learning in matrix and stochastic games when each learning agent has only minimum knowledge about the underlying game and the other learning agents. The proposed algorithms use the Exponential Moving Average (EMA) approach along with the Q-learning algorithm as a basis to update the policy for the learning agent so that the agent's policy converges to a Nash equilibrium policy. The proposed algorithms are the constant learning rate-based exponential moving average Q-learning (CLR-EMAQL) algorithm, and the exponential moving average Q-learning (EMAQL) algorithm. The proposed CLR-EMAQL algorithm uses a constant learning rate when updating the policy of the learning agent, while the proposed EMAQL algorithm uses two different decaying learning rates. These learning rates are updated based on either the WoLF mechanism or the WoLS mechanism. The WoLF mechanism makes the proposed EMAQL algorithm learn fast when the algorithm is losing, and learn cautiously when the algorithm is winning. On the other hand, the WoLS mechanism makes the proposed EMAQL algorithm learn fast when it is winning, and learn cautiously when it is losing. We mathematically show that both proposed algorithms converge to Nash equilibrium in games with pure Nash equilibrium. In games with mixed Nash equilibrium, our mathematical analysis shows that the proposed EMAQL algorithm converges to an equilibrium. Although our mathematical analysis does not explicitly show that the proposed EMAQL algorithm converges to a Nash equilibrium, our simulation results indicate that the proposed EMAQL algorithm does converge to Nash equilibrium. The proposed algorithms are examined on a variety of matrix and stochastic games. Simulation results show that the proposed EMAQL algorithm converges in a wider variety of situations than state-of-the-art multi-agent reinforcement learning algorithms. 
2. We propose a new fuzzy reinforcement learning algorithm to solve the problem of learning in differential games that have continuous state and action spaces. The proposed algorithm uses three FISs as function approximation systems; one is used as an actor (fuzzy logic controller, FLC), and the other two FISs are used as critics. The proposed algorithm incorporates the residual gradient value iteration algorithm in the tunning mechanism of the input and output parameters of its FISs (FLC and critics). The proposed algorithm is called the residual gradient fuzzy actor critics learning (RGFACL) algorithm. We evaluate the proposed algorithm on different pursuit-evasion differential games. Simulation results show that the proposed RGFACL algorithm outperforms the state-of-the-art fuzzy reinforcement learning algorithms.

3. We propose a new fuzzy reinforcement learning algorithm to solve the problem of capturing the evader, in a pursuit-evasion differential game, when the environment of the game is different from the training environment. The proposed algorithm increases the ability of the pursuer to capture the evader even when the environment of the game is different from the training environment. The proposed algorithm is called the Kalman filter fuzzy actor critic learning (KFFACL) algorithm. The proposed algorithm incorporates the future position of the evader predicted by a Kalman filter in the tunning mechanism of the fuzzy logic controller (FLC) of the learning pursuer so that the pursuer moves directly to the expected position of the evader, where the capture of the evader will occur. Moving directly to the predicted position of the evader will help the pursuer to eliminate or reduce the effect of the maneuverability of the evader and the environmental changes that may occur. We evaluate the proposed algorithm on different single pursuit-evasion differential games. Each game of these pursuit-evasion differential games has an environment different from the 
training environment the pursuer was trained on. Simulation results show the practical performance of the proposed algorithm compared to the performances of the state-of-the-art fuzzy reinforcement learning algorithms.

4. We propose a new fuzzy reinforcement learning algorithm to solve the problem of capturing a superior evader in multi-pursuer pursuit-evasion differential games with a single-superior evader that has equal speed to the speed of the pursuers. The proposed algorithm uses the RGFACL algorithm to tune the parameters of the FLC of the learning pursuer. The proposed algorithm is a decentralized algorithm as each learning pursuer has its own learning algorithm and no communication among pursuers is required. The only information the proposed learning algorithm of the learning pursuer requires is the position of the superior evader. The proposed algorithm incorporates a formation control mechanism in the reward function of the learning agent so that the pursuers are distributed around the superior evader in order to avoid collision between pursuers. In addition, the formation control mechanism used by the proposed algorithm guarantees that the capture regions of each two adjacent pursuers overlap or at least border each other so that the capture of the superior evader will be guaranteed. Our simulation results indicate that the superior evader is always captured by one or some of the pursuers learning their strategies by the proposed algorithm.

5. We propose a new decentralized fuzzy reinforcement learning algorithm to solve the problem of capturing a superior evader in multi-pursuer pursuit-evasion differential games with a single-superior evader that has a speed that is equal to or higher than the speed of each pursuer. The proposed algorithm is a decentralized algorithm as no communication among the pursuers is required. The only information the proposed algorithm requires is the position and the 
speed of the superior evader. The proposed algorithm uses the well-known Apollonius circle mechanism to define the capture region of the learning pursuer based on its location and the location of the superior evader. The proposed algorithm incorporates the Apollonius circle with a developed formation control mechanism in the reward function of the learning pursuer. This is to distribute the pursuers around the superior evader in order to avoid collision between pursuers. The formation control mechanism used by the proposed algorithm also makes the Apollonius circles of each two adjacent pursuers intersect or at least be tangent to each other so that the capture of the superior evader can occur. We evaluate the proposed algorithm on different pursuit-evasion differential games with a single-superior evader that has a speed that is equal to or higher than the speed of each pursuer. Simulation results show the effectiveness of the proposed algorithm. 


\section{List of References}

[1] S. Abdallah and V. Lesser, A multiagent reinforcement learning algorithm with non-linear dynamics, Journal of Artificial Intelligence Research 33, pp. 521-549, 2008.

[2] M. D. Awheda and H. M. Schwartz, Exponential moving average based multiagent reinforcement learning algorithms, Artificial Intelligence Review, 45.3, pp. 299-332, 2016.

[3] H. M. Schwartz, Multi-agent machine learning: A reinforcement approach, John Wiley and Sons, Inc., New York, 2014.

[4] M. D. Awheda and H. M. Schwartz, Exponential moving average Q-learning algorithm, Adaptive Dynamic Programming And Reinforcement Learning (ADPRL), IEEE Symposium on, IEEE, pp. 31-38, 2013.

[5] R. Bellman, Dynamic programming, Princeton University Press, 1957.

[6] R. A. DeCarlo, Linear systems: A state variable approach with numerical implementation, Upper SaddleRiver, New York, Prentice-Hall, 1989.

[7] H. D'Angelo, Linear time-varying systems: Analysis and synthesis, Newton, MA: Allyn \& Bacon, 1970.

[8] M. Bowling, Convergence and no-regret in multiagent learning, In Advances in Neural Information Processing Systems 17. MIT Press, 2005.

[9] M. Bowling and M. Veloso, Multiagent learning using a variable learning rate, Artificial Intelligence, 136.2, pp. 215-250, 2002.

[10] L. Buşoniu, R. Babuška, and B. D. Schutter, Multiagent reinforcement learning: A survey, 9th International Conference on Control, Automation, Robotics and Vision (ICARCV), pp. 1-6, 2006. 
[11] L. Buşoniu, R. Babuška, and B. D. Schutter, A comprehensive survey of multiagent reinforcement learning, IEEE Trans Syst Man Cybern C, 38.2, pp. 156-172, 2008.

[12] M. Thathachar and P. Sastry, Networks of learning automata: Techniques for online stochastic optimization, Boston, Massachusetts: Kluwer Academic Publishers, 2004.

[13] A. Burkov and B. Chaib-draa, Effective learning in the presence of adaptive counterparts, Journal of Algorithms, 64.4, pp. 127-138, 2009.

[14] V. Conitzer and T. Sandholm, Awesome: A general multiagent learning algorithm that converges in self-play and learns a best response against stationary opponents, Machine Learning, 67.1-2, pp. 23-43, 2007.

[15] J. Hu and M. P. Wellman, Multiagent reinforcement learning: Theoretical framework and an algorithm, In proceedings fifteenth international conference on machine learning, pp. 242-250, 1998.

[16] J. Hu and M. P. Wellman, Nash q-learning for general-sum stochastic games, Journal of Machine Learning Research, 4, pp. 1039-1069, 2003.

[17] T. Jaakkola, M. I. Jordan, and P. S. Singh, On the convergence of stochastic iterative dynamic programming algorithms, Neural Computation, 6.6, pp. 11851201, 1994.

[18] S. Sen and G. Weiss, Learning in multiagent systems, In: Multiagent Systems: A Modern Approach to Distributed Artificial Intelligence. The MIT Press, Cambridge, 1999.

[19] S. Singh, M. Kearns, and Y. Mansour, Nash convergence of gradient dynamics in general-sum games, In Proceedings of the Conference on Uncertainty in Artificial Intelligence, pp. 541-548, 2000.

[20] R. S. Sutton and A. G. Barto, Reinforcement learning: an introduction, The MIT Press, Cambridge, Massachusetts, 1998.

[21] G. Tesauro, Extending q-learning to general adaptive multi-agent systems, In Advances in Neural Information Processing Systems, 16, pp. 215-250, 2004.

[22] C. Zhang and V. Lesser, Multi-agent learning with policy prediction, In Proceedings of the 24th National Conference on Artificial Intelligence (AAAI10), Atlanta, GA, USA, pp. 746-752, 2010. 
[23] N. Fulda and D. Ventura Predicting and preventing coordination problems in cooperative learning systems, In Proceedings of the International Joint Conference on Artificial Intelligence (IJCAI-07), 2007.

[24] C. Watkins, Learning from delayed rewards, Ph.D. thesis, King's College, Cambridge, UK, 1989.

[25] C. Watkins and P. Dayan, Q-learning, Machine Learning, 8.3, pp. 279-292, 1992.

[26] S. Sen, M. Sekaran and J. Hale, Learning to coordinate without sharing information, in: Proc. AAAI-94, Seattle, WA, pp. 426-431, 1994.

[27] M. Tan, Multi-agent reinforcement learning: Independent vs. cooperative agents, in: Proc. 10th International Conference on Machine Learning, Amherst, MA, pp. 330-337, 1993.

[28] P. Sastry, V. Phansalkar, and M. Thathachar, Decentralized learning of Nash equilibria in multi-person stochastic games with incomplete information, IEEE Trans. Syst., Man, Cybern., 24.5, pp. 769-777, 1994.

[29] P. Stone and M. Veloso, Multiagent systems: A survey from a machine learning perspective, Autonomous Robots, 8.3, pp. 345-383, 2000.

[30] S. Lakshmivarahan and K. S. Narendra, Learning algorithms for two-person zerosum stochastic games with incomplete information, Mathematics of Operations Research, 6.3, pp. 379-386, 1981.

[31] S. Lakshmivarahan and K. S. Narendra, Learning algorithms for two-person zerosum stochastic games with incomplete information: a unified approach, SIAM Journal on Control and Optimization, 20.4, pp. 541-552, 1982.

[32] P. Vrancx, K. Verbeeck, and A. Nowē, Decentralized learning in markov games, IEEE Trans Syst Man Cybern B, 38.4, pp. 976-981, 2008.

[33] J. von Neumann and O. Morgenstern, Theory of games and economic behavior, John Wiley and Sons, 1944.

[34] M. J. Osborne and A. Rubinstein, A course in game theory, The MIT Press, 1994.

[35] M. Bowling, Multiagent learning in the presence of agents with limitations, $\mathrm{PhD}$ thesis, School of Computer Science, Carnegie Mellon University, Pittsburgh, PA, May 2003. 
[36] X. Lu, Multi-agent reinforcement learning in games, PhD thesis, Carleton University, Ottawa, 2012.

[37] X. Lu and H. M. Schwartz, An investigation of guarding a territory problem in a grid world, American Control Conference, P3204-3210, 2010.

[38] E. Yang and D. Gu, A survey on multiagent reinforcement learning towards multirobot systems, proceedings of IEEE symposium on computational intelligence and games, 2005.

[39] C. Claus and C. Boutilier, The dynamics of reinforcement learning in cooperative multiagent systems, AAAI/IAAI, pp. 746-752, 1998.

[40] B. Banerjee and J. Peng, Generalized multiagent learning with performance bound, Autonomous Agents and Multi-Agent Systems, 15.3, pp. 281-312, 2007.

[41] M. Bowling and M. Michael, Convergence of gradient dynamics with a variable learning rate, ICML, pp. 27-34, 2001.

[42] M. Bowling and M. Michael, Rational and convergent learning in stochastic games, International joint conference on artificial intelligence, 17.1, pp. 1021-1026, LAWRENCE ERLBAUM ASSOCIATES LTD, 2001.

[43] D. Fudenberg and D. K. Levine, The theory of learning in games, 2, MIT press, 1998.

[44] T. Basar, Tamer and G. J. Olsder, Dynamic noncooperative game theory, 200, London: Academic press, 1995.

[45] M. L. Littman, Markov games as a framework for multi-agent reinforcement learning, in Proceedings of the 11th International Conference on Machine Learning, pp. 157-163, 1994.

[46] A. W. Moore and C. G. Atkeson, Prioritized sweeping: Reinforcement learning with less data and less real time, Machine Learning, 13, pp. 103-130, 1993.

[47] R. S. Sutton, Integrated architecture for learning, planning, and reacting based on approximating dynamic programming, In Proceedings of the Seventh International Conference on Machine Learning, pp. 216-224, 1990.

[48] S. N. Givigi, H. M. Schwartz, and X. Lu, A reinforcement learning adaptive fuzzy controller for differential games, Journal of Intelligent and Robotic Systems, 59, pp. 3-30, 2010. 
[49] N. Kovvali, M. Banavar, and A. Spanias, An introduction to kalman filtering with MATLAB examples, Synthesis Lectures on Signal Processing 6.2, pp. 1-81, 2013.

[50] D. Simon, Optimal state estimation: Kalman, $H_{\infty}$, and nonlinear approaches, Wiley-Interscience, Hoboken, N.J., 2006.

[51] S. Haykin, Adaptive filter theory, 4th ed., Prentice Hall, 2001.

[52] N. M. Stiffler and J. M. O'Kane, A complete algorithm for visibility-based pursuitevasion with multiple pursuers, In Robotics and Automation (ICRA), IEEE International Conference on, pp. 1660-1667, 2014.

[53] C. Jun, S. Bhattacharya, and R. Ghrist, Pursuit-evasion game for normal distributions, In Intelligent Robots and Systems (IROS 2014), IEEE/RSJ International Conference on, pp. 83-88, 2014.

[54] A. Festa and R. B. Vinter, A decomposition technique for pursuit evasion games with many pursuers, In Decision and Control (CDC), IEEE 52nd Annual Conference on, pp. 5797-5802, 2013.

[55] E. Bakolas, Evasion from a group of pursuers with double integrator kinematics, In Decision and Control (CDC), IEEE 52nd Annual Conference on, pp. 1472-1477, 2013.

[56] M. Pachter, E. Garcia, and D. W. Casbeer, Active target defense differential game, In Communication, Control, and Computing (Allerton), 52nd Annual Allerton Conference on, pp. 46-53, 2014.

[57] S. Bhattacharya, T. Basar, and M. Falcone, Numerical approximation for a visibility based pursuit-evasion game, In Intelligent Robots and Systems (IROS), IEEE/RSJ International Conference on, pp. 68-75, 2014.

[58] N. M. Stiffler and J. M. O'Kane, A sampling-based algorithm for multirobot visibility-based pursuit-evasion, In Intelligent Robots and Systems (IROS), IEEE/RSJ International Conference on, pp. 1782-1789, 2014.

[59] D. W. Oyler, P. T. Kabamba, and A. R. Girard, Pursuit-evasion games in the presence of a line segment obstacle, In Decision and Control (CDC), IEEE 53rd Annual Conference on, pp. 1149-1154, 2014. 
[60] I. Exarchos and P. Tsiotras, An asymmetric version of the two car pursuitevasion game, In Decision and Control (CDC), IEEE 53rd Annual Conference on, pp. 4272-4277, 2014.

[61] I. Becerra, V. Macias, and R. Murrieta-Cid, On the value of information in a differential pursuit-evasion game, In Robotics and Automation (ICRA), IEEE International Conference on, pp. 4768-4774, 2015.

[62] W. Lin, Z. Qu, and M. Simaan, Nash strategies for pursuit-evasion differential games involving limited observations, Aerospace and Electronic Systems, IEEE Transactions on 51.2, pp. 1347-1356, 2015.

[63] W. Scott and N. E. Leonard, Dynamics of pursuit and evasion in a heterogeneous herd, In Decision and Control (CDC), IEEE 53rd Annual Conference on, pp. 29202925, IEEE, 2014.

[64] A. Kumar and A. Ojha, An evader-centric strategy against fast pursuer in an unknown environment with static obstacles, In Control, Automation, Robotics and Embedded Systems (CARE), International Conference on, pp. 1-6, IEEE, 2013.

[65] J. Dong, X. Zhang, and X. Jia, Strategies of Pursuit-Evasion Game Based on Improved Potential Field and Differential Game Theory for Mobile Robots, In Instrumentation, Measurement, Computer, Communication and Control (IMCCC), Second International Conference on, pp. 1452-1456, IEEE, 2012.

[66] W. Li, A Dynamics Perspective of Pursuit-Evasion: Capturing and Escaping When The Pursuer Runs Faster Than The Agile Evader, IEEE Transaction on Automatic Control, 99, 2016.

[67] J. Selvakumar and E. Bakolas, Evasion from a group of pursuers with a prescribed target set for the evader, American Control Conference, 2016.

[68] Q. Wang and M. Liu, Learning in Hide-and-Seek, IEEE/ACM Transaction on Networking, 24.2, 2016.

[69] W. Li, Escape Analysis on the Confinement-Escape Problem of a Defender Against an Evader Escaping From a Circular Region, IEEE Transaction on Cybernetics, 46.9, 2016.

[70] W. Li, The Confinement-Escape Problem of a Defender Against an Evader Escaping from a Circular Region, IEEE Transaction on Cybernetics, 46.4, 2016. 
[71] A. Alexopoulos and E. Badreddin, Decomposition of multi-player games on the example of pursuit-evasion games with unmanned aerial vehicles, American Control Conference, 2016.

[72] X, Wang, J. B. Cruz Jr, G. Chen, K. Pham, and E. Blasch, Formation control in multi-player pursuit evasion game with superior evaders, In Defense and Security Symposium, International Society for Optics and Photonics, 2007.

[73] M. Wei, G. Chen, J. B. Cruz, L. S. Haynes, M. H. Chang and E. Blasch, A decentralized approach to pursuer-evader games with multiple superior evaders in noisy environments, In Aerospace Conference, 2007.

[74] S. Jin and Z. Qu, Pursuit-evasion games with multi-pursuer vs. one fast evader, In Intelligent Control and Automation (WCICA), 8th World Congress, 2010.

[75] M. Wei, G. Chen, J. B. Cruz, L. Hayes and M. H. Chang, A decentralized approach to pursuer-evader games with multiple superior evaders, In Intelligent Transportation Systems Conference, pp. 1586-1591, 2006.

[76] D. Li and J. B. Cruz, Better cooperative control with limited look-ahead, In American Control Conference, 2006.

[77] D. Li, J. B. Cruz, G. Chen, C. Kwan and M. H. Chang, A hierarchical approach to multi-player pursuit-evasion differential games, In Decision and Control, European Control Conference, CDC-ECC'05, 44th IEEE Conference on, pp. 5674-5679, 2005.

[78] M. Wei, G. Chen, J. B. Cruz, L. Haynes, K. Pham, K. and E. Blasch, Multipursuer multi-evader pursuit-evasion games with jamming confrontation, Journal of Aerospace Computing, Information, and Communication, 4.3, pp. 693-706, 2007.

[79] Z S. Cai, L. N. Sun and H. B. Gao, A novel hierarchical decomposition for multiplayer pursuit evasion differential game with superior evaders, In Proceedings of the first ACM/SIGEVO Summit on Genetic and Evolutionary Computation, pp. 795-798, ACM, 2009.

[80] F. Bao-Fu, P. Qi-Shu, H. Bing-Rong, D. Lei, Z. Qiu-Bo and Z. Zhaosheng, Research on high speed evader vs. multi lower speed pursuers in multi pursuit-evasion games, Information Technology Journal, 11.8, 2012. 
[81] H. Wang, Q. Yue, and J. Liu, Research on Pursuit-evasion games with multiple heterogeneous pursuers and a high speed evader, Control and Decision Conference (CCDC), 27th Chinese, IEEE, 2015.

[82] S. Jin and Z. Qu, A heuristic task scheduling for multi-pursuer multi-evader games, Information and Automation (ICIA), IEEE International Conference on, 2011.

[83] M. Kothari, J. G. Manathara, and I. Postlethwaite, A Cooperative PursuitEvasion Game for Non-holonomic Systems, World Congress, 19.1, 2014.

[84] H. V. Hasselt and M. Wiering, Reinforcement learning in continuous action spaces, Approximate Dynamic Programming and Reinforcement Learning, ADPRL 2007, IEEE International Symposium on, 2007.

[85] K. Doya, Reinforcement learning in continuous time and space, Neural computation 12.1, pp. 219-245, 2000.

[86] W. D. Smart and L. P. Kaelbling, Practical reinforcement learning in continuous spaces, ICML, 2000.

[87] A. Lazaric, M. Restelli, and A. Bonarini, Reinforcement learning in continuous action spaces through sequential Monte Carlo methods, Advances in neural information processing systems, 2007.

[88] Z. Xia and D. Zhao, Online reinforcement learning control by Bayesian inference, IET Control Theory \& Applications, 10.12, pp. 1331-1338, 2016.

[89] Y. J. Liu, Y. Gao, S. Tong, and Y. Li, Fuzzy approximation-based adaptive backstepping optimal control for a class of nonlinear discrete-time systems with deadzone, IEEE Transactions on Fuzzy Systems, 24.1, pp. 16-28, 2016.

[90] Y. Zhu, D. Zhao, and X. Li, Using reinforcement learning techniques to solve continuous-time non-linear optimal tracking problem without system dynamics, IET Control Theory \& Applications, 10.12, pp. 1339-1347, 2016.

[91] R. Kamalapurkar, P. Walters, and W. E. Dixon, Model-based reinforcement learning for approximate optimal regulation, Automatica, 64, pp. 94-104, 2016.

[92] H. Jiang, H. Zhang, Y. Luo, and J. Wang, Optimal tracking control for completely unknown nonlinear discrete-time Markov jump systems using data-based reinforcement learning method, Neurocomputing, 194, pp. 176-182, 2016. 
[93] S. Micera, A. M. Sabatini, and P. Dario. Adaptive fuzzy control of electrically stimulated muscles for arm movements, Medical \& biological engineering \& computing, 37.6, pp. 680-685, 1999.

[94] F. Daldaban, N. Ustkoyuncu, and K. Guney, Phase inductance estimation for switched reluctance motor using adaptive neuro- fuzzy inference system, Energy Conversion and Management, 47.5, pp. 485-493, 2005.

[95] S. Labiod and T. M. Guerra, Adaptive fuzzy control of a class of SISO nonaffine nonlinear systems, Fuzzy Sets and Systems, 158.10, pp. 1126-1137, 2007.

[96] H. K. Lam and FH F. Leung, Fuzzy controller with stability and performance rules for nonlinear systems, Fuzzy Sets and Systems 158.2, pp. 147-163, 2007.

[97] H. Hagras, V. Callaghan, and M. Colley, Learning and adaptation of an intelligent mobile robot navigator operating in unstructured environment based on a novel online Fuzzy-Genetic system, Fuzzy Sets and Systems, 141.1, pp. 107-160, 2004.

[98] M. Mucientes, D. L. Moreno, A. Bugarn S. and Barro,Design of a fuzzy controller in mobile robotics using genetic algorithms, Applied Soft Computing, 7.2, pp. 540546, 2007.

[99] L. Jouffe, Fuzzy inference system learning by reinforcement methods, IEEE Trans. Syst., Man, Cybern. C, 28.3, pp. 338-355, 1998.

[100] S. F. Desouky and H. M. Schwartz, $Q(\lambda)$-learning adaptive fuzzy logic controllers for pursuit-evasion differential games, International Journal of Adaptive Control and Signal Processing, 25.10, pp. 910-927, 2011.

[101] M. D. Awheda and H. M. Schwartz, The residual gradient FACL algorithm for differential games, Electrical and Computer Engineering (CCECE), IEEE 28th Canadian Conference on, pp. 1006-1011, 2015.

[102] M. D. Awheda and H. M. Schwartz, A residual gradient fuzzy reinforcement learning algorithm for differential games, International Journal of Fuzzy Systems, Accepted for publication, Oct. 2016.

[103] W. Hinojosa, S. Nefti and U. Kaymak, Systems control with generalized probabilistic fuzzy-reinforcement learning, Fuzzy Systems, IEEE Transactions on, 19.1, pp. 51-64, 2011. 
[104] M. Rodríguez, R. Iglesias, C. V. Regueiro and J. Correa and S. Barro, Autonomous and fast robot learning through motivation, Robotics and Autonomous Systems, 55.9, pp. 735-740, 2007.

[105] M. D. Awheda and H. M. Schwartz, A fuzzy reinforcement learning algorithm using a predictor for pursuit-evasion games, Proceedings of the 2016 IEEE International Systems Conference, 2016.

[106] B. Luo, H.N. Wu and T. Huang, Off-policy reinforcement learning for $H_{\infty}$ control design, Cybernetics, IEEE Transactions on, 45.1, pp. 65-76, 2015.

[107] B. Luo, H.N. Wu and H.X. Li, Adaptive optimal control of highly dissipative nonlinear spatially distributed processes with neuro-dynamic programming, Neural Networks and Learning Systems, IEEE Transactions on, 26.4, pp. 684-696, 2015.

[108] B. Luo, H.N. Wu, T. Huang and D. Liu, Reinforcement learning solution for HJB equation arising in constrained optimal control problem, Neural Networks, 71, pp. 150-158, 2015.

[109] H. Modares, F. L. Lewis and M.B. Naghibi-Sistani, Integral reinforcement learning and experience replay for adaptive optimal control of partially-unknown constrained-input continuous-time systems, Automatica, 50.1, pp. 193-202, 2014.

[110] W. Dixon, Optimal adaptive control and differential games by reinforcement learning principles, Journal of Guidance, Control, and Dynamics, 37.3, pp. 10481049, American Institute of Aeronautics and Astronautics, 2014.

[111] B. Luo, H.N. Wu and H.X. Li, Data-based suboptimal neuro-control design with reinforcement learning for dissipative spatially distributed processes, Industrial \& Engineering Chemistry Research, 53.19, pp. 8106-8119, 2014.

[112] H.N. Wu and B. Luo, Neural network based online simultaneous policy update algorithm for solving the HJI equation in nonlinear control, Neural Networks and Learning Systems, IEEE Transactions on, 23.12, pp. 1884-1895, 2012.

[113] L. Baird, Residual algorithms: Reinforcement learning with function approximation, In ICML, pp. 30-37, 1995.

[114] L. P. Kaelbling, M. L. Littman and A. W. Moore, Reinforcement learning: A survey, arXiv preprint cs/9605103 (1996). 
[115] W. D. Smart and L. P. Kaelbling, Effective reinforcement learning for mobile robots, IEEE International Conference on Robotics and Automation, Proceedings ICRA'02, 4, 2002.

[116] C. Ye, N. HC Yung, and D. Wang, A fuzzy controller with supervised learning assisted reinforcement learning algorithm for obstacle avoidance, Systems, Man, and Cybernetics, Part B: Cybernetics, IEEE Transactions on, 33.1, pp. 17-27, 2003.

[117] T. Kondo and K. Ito, A reinforcement learning with revolutionary state recruitment strategy for autonomous mobile robots control, Robotics and Autonomous Systems, 46, pp. 111-124, 2004.

[118] D. A. Gutnisky and B. S. Zanutto, Learning obstacle avoidance with an operant behavior model, Artificial Life, 10.1, pp. 65-81, 2004.

[119] R. S. Sutton, Learning to predict by the methods of temporal differences, Machine learning, 3.1, pp. 9-44, 1988.

[120] J. W. Sheppard, Colearning in differential games, Machine Learning, 33, pp. 201-233, 1998.

[121] S. F. Desouky and H. M. Schwartz, Self-learning fuzzy logic controllers for pursuit-evasion differential games, Robotics and Autonomous Systems, 59, pp. 22-33, 2011.

[122] W. M. Van Buijtenen, G. Schram, R. Babuska, and H. B. Verbruggen, Adaptive fuzzy control of satellite attitude by reinforcement learning, Fuzzy Systems, IEEE Transactions on, 6.2, pp. 185-194, 1998.

[123] A. Bonarini, A. Lazaric, F. Montrone, and M. Restelli, Reinforcement distribution in fuzzy Q-learning, Fuzzy sets and systems, 160.10, pp. 1420-1443, 2009.

[124] P. Dayan and T. J. Sejnowski, TD $(\lambda)$ converges with probability 1, Machine Learning, 14, pp. 295-301, 1994.

[125] P. Dayan, The convergence of TD $(\lambda)$ for general $\lambda$, Machine Learning 8.3/4, pp. 341-362, 1992.

[126] X. Dai, C. Li, and A. B. Rad, An approach to tune fuzzy controllers based on reinforcement learning for autonomous vehicle control, IEEE Transactions on Intelligent Transportation Systems, 6.3, pp. 285-293, 2005. 
[127] X.S. Wang, Y.H. Cheng, and J.Q. Yi, A fuzzy Actor-Critic reinforcement learning network, Information Sciences, 177.18, pp. 3764-3781, 2007.

[128] A. G. Barto, R. S. Sutton and C. W. Anderson, Neuronlike adaptive elements that can solve difficult learning control problems, Systems, Man and Cybernetics, IEEE Transactions on, 5, pp. 834-846, 1983.

[129] J. Boyan, A. W. Moore, Generalization in reinforcement learning: Safely approximating the value function, Advances in neural information processing systems, pp. 369-376, 1995.

[130] G. J. Gordon, Reinforcement learning with function approximation converges to a region, Advances in neural information processing systems, 2001.

[131] R. Schoknecht and A. Merke, TD(0) converges provably faster than the residual gradient algorithm, ICML, 2003.

[132] J. N. Tsitsiklis and B. V. Roy, An analysis of temporal-difference learning with function approximation, Automatic Control, IEEE Transactions on, 42.5, pp. 674690, 1997.

[133] R. Isaacs, Differential game, John Wiley and Sons, 1965.

[134] S. M. LaValle, Planning algorithms, Cambridge University Press, 2006.

[135] S. H. Lim, T. Furukawa, G. Dissanayake and H.F.D. Whyte, A time-optimal control strategy for pursuit-evasion games problems, In International Conference on Robotics and Automation, New Orleans, LA, 2004.

[136] S. F. Desouky and H. M. Schwartz, Different hybrid intelligent systems applied for the pursuit-evasion game, In 2009 IEEE International Conference on Systems, Man, and Cybernetics, pp. 2677-2682, 2009.

[137] S. Soga and I. Kobayashi, A study on the efficiency of learning a robot controller in various environments, In Adaptive Dynamic Programming And Reinforcement Learning (ADPRL), IEEE Symposium on, pp. 164-169, 2013.

[138] M. D. Awheda and H. M. Schwartz, Decentralized learning in pursuit-evasion differential games with multi-pursuer and single-superior evader, Proceedings of the 2016 IEEE International Systems Conference, 2016.

[139] M. D. Awheda and H. M. Schwartz, A decentralized fuzzy learning algorithm for pursuit-evasion differential games with superior evaders, Journal of Intelligent and Robotic Systems, 83.1, pp. 35-53, 2016. 
[140] J.S. R. Jang and C.T. Sun, Neuro-fuzzy and soft computing: a computational approach to learning and machine intelligence, Prentice-Hall, Inc., Upper Saddle River, NJ, USA, 1997.

[141] L.X. Wang, A course in fuzzy systems and control, Prentice-Hall, Inc., Upper Saddle River, NJ, USA, 1997.

[142] T. J. Ross, Fuzzy Logic with Engineering Applications, John Wiley and Sons, Ltd, 2010.

[143] K. M. Passino and S. Yurkovich, Fuzzy control, Addison Wesley Longman, 1998.

[144] J. Jantzen, Design of fuzzy controllers, Technical Report (No:98-E864) Department of Automation, Technical Univ. of Denmark, 1999.

[145] E. H. Mamdani and S. Assilian, An experiment in linguistic synthesis with a fuzzy logic controller, International Journal of Man-Machine Studies, 7.1, pp. 1-13, 1975.

[146] T. Takagi and M. Sugeno, Fuzzy identification of systems and its applications to modelling and control, IEEE Transactions on Systems, Man and Cybernetics, SMC, 15.1, pp. 116-132, 1985.

[147] M. Sugeno and G. Kang, Structure identification of fuzzy model, Fuzzy Sets Syst., 28, pp. 15-33, 1988. 